\title{
MIGRATION AND NATURAL FATE OF A COAL TAR CREOSOTE PLUME
}

\author{
by
}

\section{Mark William George King}

\author{
A thesis \\ presented to the University of Waterloo \\ in fulfilment of the \\ thesis requirement for the degree of \\ Doctor of Philosophy \\ in \\ Earth Sciences
}

Waterloo, Ontario, Canada, 1997

(C) Mark King, 1997 
National Library

of Canada

Acquisitions and Bibliographic Services

395 Wellington Sreet Otrawe ON KIA ONA Canada
Bibliothèque nationale du Canada

Acquisitions et services bibliographiques

395, ne Wellington

Otrewr ON KIA ON

Canada

Your ste vosenenturences

Our sis Nowe restrence

The author has granted a nonexclusive licence allowing the National Library of Canada to reproduce, loan, distribute or sell copies of his/her thesis by any means and in any form or format, making this thesis available to interested persons.

The author retains ownership of the copyright in his/her thesis. Neither the thesis nor substantial extracts from it may be printed or otherwise reproduced with the author's permission.
L'auteur a accordé une licence non exclusive permettant à la Bibliothèque nationale du Canada de reproduire, prêter, distribuer ou vendre des copies de sa thèse de quelque manière et sous quelque forme que ce soit pour mettre des exemplaires de cette thèse à la disposition des persomnes intéressées.

L'auteur conserve la propriété du droit d'auteur qui protège sa thèse. Ni la thèse ni des extraits substantiels de celle-ci ne doivent être imprimés ou autrement reproduits sans son autorisation. 
The University of Waterloo requires the signatures of all persons using or photocopying this thesis. Please sign below, and give address and date. 


\begin{abstract}
A volume of sand containing coal tar creosote was emplaced below the water table at CFB Borden to investigate natural attenuation processes for complex biodegradable mixtures. Coal tar creosote is a mixture of more than 200 polycyclic aromatic hydrocarbons, heterocyclic compounds and phenolic compounds. A representative group of seven compounds was selected for detailed study: phenol, $m$-xylene, naphthalene, phenanthrene, 1-methylnaphthalene, dibenzofuran and carbazole. Movement of groundwater through the source led to the development of a dissolved organic plume, which was studied over a four year period.

Qualitative plume observations and mass balance calculations indicated two key conclusions: 1) compounds from the same source can display distinctly different patterns of plume development and 2) mass transformation was a major influence on plume behaviour for all observed compounds. After being completely leached from the source early in the study, phenol migrated as a discrete slug plume and almost completely disappeared after two years. The $m$-xylene plume migrated outward to a maximum distance at approximately two years, and then receded back towards the source as the rate of mass flux out of the source decreased to below the overall rate of plume transformation. Carbazole showed similar behaviour, although the reversal in plume development occurred more slowly.
\end{abstract}

The dibenzofuran plume remained relatively constant in extent and mass over the last two years of monitoring, despite that constant source input over this period. This indicated that the rate of mass input was approximately equal to the rate of plume transformation and is the first conclusive documentation of a "steady state" plume, of which the author is aware. Meanwhile, the naphthalene and 1-methylnaphthalene plume continued to advance over the observation period, although decreases in the rate of mass input from the source to the plume were noted for both. The phenanthrene plume was also subject to transformation, although measurement of the rate was less conclusive due the higher proportion of sorbed mass for this compound.

Multiple lines of evidence were used to evaluate whether the observed plume mass loss was due to microbial biodegradation. Comparison of sterile and active laboratory microcosm using aquifer material indicated that aquifer microbes were able to metabolize plume compounds.

Measurement of redox-sensitive parameters in the vicinity of the plume showed the types of changes that would be expected to occur due to plume biodegradation: dissolved oxygen and $\mathrm{SO}_{4}^{2-}$ decreased in groundwater within the plume while significant increases were noted for $\mathrm{Fe}^{2+}$, $\mathrm{Mn}^{2+}$ and methane. Further evidence that plume mass loss was microbially-mediated was provided by the accumulation of aromatic acids within the plume. Measurements of phospholipid fatty acids (PLFA) in aquifer material indicated that microbial biomass and turnover rate were greater within the plume: also consistent with biodegradation.

Computer modelling confirmed that mass transformation was a strong influence on plume behaviour. It also indicated that the observed mass loss was far greater than would be expected due to aerobic respiration leading to complete mineralization. A mass balance of the change in 
electron acceptor against organics mass loss showed that $\mathrm{SO}_{4}^{2-}$ was almost as important as dissolved oxygen, in terms of utilization for plume transformation. However, the total change in electron acceptors was still substantially smaller than that required for complete mineralization of plume compounds. This discrepancy was qualitatively explained by the measured occurrence of organic metabolites. With the production of these organic intermediates, transformation of plume organics may require an unexpectedly small quantity of electron acceptor. This points to a major weakness in modelling biodegradation with a stoichiometric approach that assumes complete mineralization. 


\section{Acknowledgments}

The research described herein has taken a multi-disciplinary approach and has been conducted as part of a larger research effort, the Coal Tar Organics in Groundwater Program. Consequently, it has provided me with the opportunity to work with a large number of people, all of whom are highly competent and enthusiastic in their respective fields.

My Supervisor, James Barker, tops the list of those to which I express my gratitude. Jim has allowed me considerable control over the direction of this research while, at the same time, keeping the project interesting and on track by giving freely of himself as a technical resource and "reality check". I also thank the other members of my committee for thoughtful reviews of the work: Rick Devlin, Barb Butler, John Cherry, Neil Thompson and Michael Barcelona.

Several UW Earth Science Department staff members have been invaluable to this project. Kim Hamilton has spent countless days involved in laboratory analysis and field sampling, and was never daunted by the seemingly endless stream of samples. The expertise of Paul Johnson in coring and piezometer installation was greatly appreciated, as was that of John Molson in computer modelling. The efforts and cooperation of Stephanie O'Hannesin, Greg Friday, Shirley Chatten, Mark Walker and Tracy Fowler are also gratefully acknowledged.

The microbial components of the study would not have been possible without the input of Barb Butler and Mary Ann Vandergreindt of the UW Biology Department, and I am grateful to them for tolerating a hydrogeologist in their laboratory. I also thank previous students in the CoalTar Organics Program: Heather Malcolmson for initiating the field experiment; Jeanet Nielsen and Susanne Hansen for collecting some of the early data.

A stalwart group of fellow students have braved the mosquitoes, rain and heat of Borden to render assistance in the plume sampling program. For this I thank Shaheed Rahman, Jim Roy, Kathy Main, Steve Forsey, Jennifer Maude, Everton de Oliviera, Stan Beaubien, Parminder Singh and Michelle Bester. I also thank fellow students Mario Schirmer and Uli Mayer for generously sharing their expertise in computer modelling. Jan Erik Sorlie and Jeff Barbaro, with whom I shared an office (at different times) during my years in Waterloo, provided much appreciated support in the form of ongoing informal discussions.

I gratefully acknowledge the useful analyses and input by individuals from two outside agencies: Michael Barcelona and Jiasong Fang at the National Center for Integrated Bioremediation Research and Development (University of Michigan) and Andrew White from Microbial Insights (Knoxville, Tennessee).

Finally, major funding for this research was provided by the Natural Sciences and Engineering Research Council, in the form of a Scholarship (to me) and a Strategic Operating Grant (to the project). Additional funding was provided by the Ontario Ministry of the Environment and Domtar. 


\section{Dedication}

To Laura: for making it possible to pursue this goal and for making our "Waterloo" years another in a series of excellent adventures.

To Sam and Hannah: for helping Daddy keep things in perspective.

To Mom: for providing the driving force and for eternal optimism.

To Dad: for the work ethic and for healthy skepticism. 
TABLE OF CONTENTS

Chapter

Page

1. INTRODUCTION

STUDY BACKGROUND

BIODEGRADATION OVERVIEW

ENVIRONMENTAL BIODEGRADATION OF COMPLEX MIXTURES 2

FIELD STRATEGIES AND LIMITATIONS 3

STUDY APPROACH AND PURPOSE

2. PROJECT OVERVIEW 6

SITE DESCRIPTION 6

CREOSOTE COMPOSITION AND PROPERTIES $\quad 6$

METHODS

Source Emplacement $\quad 8$

Plume Monitoring Network $\quad 10$

Plume Sampling $\quad 12$

Sample Analysis $\quad 13$

Source Composition 13

Source Dissolution and Leachate Composition $\quad 14$

Potential Sources of Error $\quad 17$

RESULTS AND DISCUSSION 19

General $\quad 19$

Chloride $\quad 20$

Phenol $\quad 29$

$M$-xylene $\quad 30$

Naphthalene $\quad 31$

Dibenzofuran 31

Phenanthrene $\quad 32$

Carbazole $\quad 32$

l-Methylnaphthalene $\quad 32$

3. MASS BALANCE 33

BACKGROUND

METHODS

General Approach $\quad 35$

Dissolved Mass $\quad 38$

Sorbed Mass $\quad 39$

Mass Flux $\quad 43$

MASS BALANCE RESULTS $\quad 46$

DISCUSSION $\quad 46$ 


\section{TABLE OF CONTENTS}

(continued)

Chapter

Page

4. BIODJGRADATION INDICATORS 50

$\begin{array}{ll}\text { BACKGROUND } & \mathbf{5 0}\end{array}$

LABORATORY MICROCOSMS 51

Methods 51

Results $\quad 53$

$\begin{array}{lr}\text { PLUME REDOX CONDITIONS } & 57\end{array}$

$\begin{array}{ll}\text { Background } & 57\end{array}$

Methods $\quad 58$

Results $\quad 59$

MICROBIOLOGICAL INDICATORS

Background $\quad 61$

$\begin{array}{ll}\text { Methods } & 62\end{array}$

Results $\quad 62$

ORGANIC METABOLITES

$\begin{array}{ll}\text { Background } & 64\end{array}$

$\begin{array}{ll}\text { Methods } & 65\end{array}$

Results $\quad 66$

5. CONCEPTUAL MODEL OF PLUME BEHAVIOUR 68

$\begin{array}{ll}\text { INTRODUCTION } & 68\end{array}$

$\begin{array}{lr}\text { CONCEPTUAL MODEL } & 69\end{array}$

$\begin{array}{ll}\text { RESULTS AND DISCUSSION } & 72\end{array}$

General Effect of Plume Biodegradation $\quad 72$

Electron Acceptors $\quad 75$

Organic Metabolites Distribution $\quad 81$

$\begin{array}{lr}\text { 6. SUMMARY AND CONCLUSIONS } & 83\end{array}$

7. RECOMMENDATIONS 86

8. REFERENCES 


\section{LIST OF TABLES}

2.1 Characteristics of the Borden aquifer 7

2.2 Background groundwater characteristics $\quad 8$

2.3 Summary of synoptic monitoring program $\quad 12$

2.4 Quantitative analysis of creosote and compound properties 14

2.5 Qualitative analysis of creosote composition 15

2.6 Summary of data for estimation of average linear groundwater velocity 29

3.1 Summary of analytical data for sand cores collected from the source 36

3.2 Estimates of solids partitioning coefficients $\left(K_{d}\right.$ 's) for selected compounds and Borden sand

3.3 Summary of mass balance input data and results 47

3.4 Half lives (in days) for selected creosote compounds 48

4.1 Mean aqueous concentrations ( $\mathrm{ppb} ; \mathrm{n}=3$ ) at the beginning and end of the microcosm experiment $\quad 55$

4.2 Results of plate count analysis for sand samples collected from microcosms 57

4.3 Summary of data for selected redox and geochemical indicator parameters 59

4.4 Results of mean concentration comparison ( $t$-test) for samples collected from within the dissolved creosote plume and background samples

4.5 List of aromatic acids and creosote compounds analyzed to evaluate for the presence of organic metabolites

4.6 Results of groundwater samples used to evaluate for the presence of creosote plume metabolites

5.1 Standard reduction potentials and free energy changes for half cell reactions of primary electron acceptors

$\begin{array}{lll}5.2 & \text { Model input parameters } & 73\end{array}$

5.3 Masses of selected compounds in the emplaced creosote source plume 75

$\begin{array}{lll}5.4 & \text { Oxidation reactions involving oxygen and selected creosote compounds } & 78\end{array}$

5.5 Oxidation reactions involving dibenzofuran and primary electron acceptors $\quad 80$

5.6 Balance of oxidation reactants or products against mass loss of creosote compounds 


\section{LIST OF FIGURES}

Page

2.1 Configuration of the creosote source 9

2.2 Location of the creosote source and plume monitoring network 11

2.3 Aqueous concentration of selected creosote compounds in groundwater leaving the emplaced source

2.4 Contour plans of chloride concentrations 21

2.5 Contour plans of phenol concentrations 22

2.6 Contour plans of $m$-xylene concentrations 23

2.7 Contour plans of naphthalene concentrations 24

2.8 Contour plans of dibenzofuran concentrations 25

2.9 Profiles of chloride and phenol concentrations 26

2.10 Profiles of dissolved concentrations of selected plume organics 27

2.11 Time series plots for chloride and selected organics concentrations 28

3.1 Conceptualized cross-section showing the main components of the mass balance for the plume from the emplaced creosote source $\quad 37$

3.2 Plan view of groundwater flowlines in the vicinity of the emplaced creosote source 44

4.1 Plan showing plume monitoring locations for selected redox parameters, aromatic acids and microbiological indicator parameters $\quad 52$

4.2 Plot of average normalized concentrations $(n=3)$ in aerobic microcosms 54

4.3 Bar graph of $\mathrm{CO}_{2}$ concentration in aerobic microcosms 56

4.4 Plan showing dissolved oxygen monitoring locations along cross-sections oriented parallel to groundwater flow (A-A') amd perpendicular (B-B'); cross-sections show distribution of dissolved oxygen relative to the dissolved creosote plume, as of Day 1357

4.5 Indicators of microbial numbers and status in aquifer material collected from within and outside the dissolved creosote plume

5.1 Plan view illustration of the conceptual model for natural attenuation of aerobically biodegradable organics in groundwater $\quad 70$

5.2 Configuration of the $20 \mathrm{ppb}$ contour for the dibenzofuran plume 74

5.3 Average concentrations of redox-related parameters in background and plume groundwater

5.4 Distribution of oxidation reactants and products at 1460 days after source emplacement

5.5 Variation in direction of groundwater hydraulic gradient at the emplaced creosote source site 


\section{LIST OF APPENDICES}

No.

Pages

1. Multi-level Sampler Specifications 96

2. Synoptic Sampling Data 97

3. Time Series Sampling Data 103

4. Creosote Analytical Protocol 104

5. Characterization of the Study Creosote 115

6. Paper presented at the APINGWA Conference, November, $1994 \quad 124$

7. Results for Quality Assurance and Quality Control 140

8. Procedure for Estimation of Partitioning Coefficients 149

9. In situ $K_{d}$ estimation 152

10. Microcosm Experiment 156

I1. Redox Characterization - Methods and Results 163

12. PLFA and Plate Counts - Methods and Results 173

13. Aromatic Acids Results 193

14. BIO3D Input Files 195 


\section{CHAPTER 1 INTRODUCTION}

\section{STUDY BACKGROUND}

Many products and wastes derived from coal and oil are mixtures composed of aromatic, heteroaromatic and phenolic compounds. These compounds span a wide range of molecular weights, from the lighter monoaromatics (e.g., BTEX) and phenolic compounds to the mid-range, two- to four-ring polycyclic aromatic hydrocarbons (PAHs) and nitrogen, sulphur and oxygen (NSO) heteroaromatics up to the larger PAHs with greater than four rings. These types of complex mixtures are used, or generated, by industries such as coal distillation, petroleum refining, wood treating and metal refining. Accidental spills or past waste disposal practices associated with these industries have caused widespread groundwater contamination by the constituent compounds, many of which are toxic or carcinogenic.

Predicting the fate and transport of this type of contamination presents unique problems. It is generally acknowledged that most of these compounds are biodegradable, to some degree, under aerobic conditions (e.g., Mueller et al., 1989). This property may serve to limit the extent of groundwater contamination since shallow groundwater typically contains detectable dissolved oxygen. However, due to the complex nature of these plumes, the extent of natural attenuation is not easily predicted. Dissolution is the first step of plume formation and is particularly complicated for mixtures with a wide range of compound solubilities. Further, as dissolution proceeds a chromatographic separation of compounds occurs along the groundwater flowpath, due to variations in the sorptive properties of the constituent compounds. Consequently, the multi-compound "plume" is effectively separated into a series of single-compound plumes, each of which may exhibit distinct sorptive characteristics. Superimposed on this process is the possibility of competition between organic compounds, for dissolved oxygen, or other electron acceptors. A further complication arises because the various compounds may have widely varying tendencies to degrade under aerobic, and anaerobic conditions.

\section{BIODEGRADATION OVERVIEW}

Evidence of widespread microbial presence in the subsurface environment is now well documented (e.g., Barbaro et al., 1994; Wilson et al., 1983; Ghiorse and Balkwill, 1983) and widely accepted. The ability of indigenous microbes, especially bacteria, to degrade a wide range of organic substances has also been conclusively demonstrated. Atlas (1981) provided some general observations about the ability of compound groups typically associated with petroleum to serve as microbial substrates. He indicated that heteroaromatic NSO compounds with a small number of rings may be biodegradable, as may monoaromatic compounds and PAHs with two to four rings. 
Mueller et al. (1989) provided an overview of biodegradation for a similar range of compounds, with reference to environmental contamination by creosote. They cited several laboratory studies where biodegradation of PAHs, heteroaromatics and phenolic compounds has been demonstrated and where specific strains of microbes able to utilize these compounds have been isolated from environmental samples. However, they pointed out that of these three groups, much less is known about biodegradation processes for the heteroaromatics.

Biodegradability of organic compounds is more easily demonstrated in the laboratory than in the field and the method most often used involves microcosms. A detailed review of general microcosm techniques is provided by Pritchard and Bourquin (1983). In general, a quantity of the compound in question is placed in a sealed vessel with some combination of soil and water. Trials are conducted with both active and sterilized microcosms so that biotic mass loss can be differentiated from abiotic losses. However, there are limitations with regard to extending these types of results to the natural systems. These are primarily related to disruption of the dynamic process that may occur in the field and to difficulty in duplicating field environmental conditions in the laboratory. Alexander (1985) also cautions that degradation rates may be highly dependent on organic compound concentrations, making it difficult to apply results from one microcosm experiment to the continuum of concentrations usually encountered in the groundwater plumes. Consequently, large-scale field experiments have potential to provide results that are more representative.

\section{ENVIRONMENTAL BIODEGRADATION OF COMPLEX MIXTURES}

In recent years, natural biodegradation has received considerable attention as a potential remediation strategy for hydrocarbon contamination of groundwater (National Research Council, 1993). This is probably a response to a combination of factors: the high cost of remediation by more active means and the poor results that have characterized these efforts, to date (e.g., review of pump-and-treat limitations by Mackay and Cherry (1989)). Given that biodegradability has been demonstrated in the laboratory for so many organics, it would appear that a strong case for using natural attenuation as a remediation strategy is already made. However, two issues remain: 1) as mentioned earlier, laboratory results may not be representative of the field and 2) even if biodegradation is occurring in the field, it may not be sufficient to isolate the contaminated site from the surrounding environment. Consequently, to use natural microbial attenuation as a remedial strategy, field biodegradation must first be proven and then quantified. Madsen (1991) has laid out guidelines for evaluating the occurrence, but not the rate, of in situ biodegradation. The approach is based on:

1. contaminant distributions that indicate diminishing concentrations in excess of hydrodynamic dilution;

2. associated changes in reactants concentrations (e.g., electron acceptors, nutrients, metabolites); and 
3. laboratory evidence demonstrating that subsurface microbes are able to utilize the organic(s) in question and field evidence of microbial distribution that correlates with contaminant distribution.

Such a unified approach was used by Klecka et al. (1990) to demonstrate natural bioremediation of PAHs and phenolic compounds in groundwater at a previous charcoal manufacturing plant in Michigan. The authors used a combination of organics and inorganics monitoring, laboratory microcosms and assessment of biological activity. Madsen et al. (1991) demonstrated natural bioremediation of PAHs at a coal tar disposal site in a shallow aquifer using a combination of groundwater monitoring for organics and inorganics and inferences from subsurface microbial ecology. The authors found a relationship between distribution of protozoa and plume organics and suggested that the propulation of the former increased because they were grazing on bacteria that were growing on plume organics.

The USGS has conducted two major studies that probably represent the most intensive characterizations of natural bioremediation processes, to date. At the site of a crude oil spill in Bemidji, Minnesota, researchers showed the development of redox zones in groundwater due to the spill as well as evolution of metabolites from biodegradation of crude oil compounds (Cozzarelli et al., 1994). The other USGS study was conducted at a former wood preserving site in Pensacola, Florida (Mattraw and Franks, 1986). Natural bioremediation of plume organics (primarily PAHs and phenols) was inferred by changes in plume inorganic geochemistry indicative of redox reactions. The occurrence of fatty acids as organic metabolites was another indication of biodegradation (Goerlitz et al., 1985). A greater than expected decrease in metabolite concentrations along the groundwater flow path was interpreted as evidence of subsequent metabolite biodegradation (Godsy et al., 1992).

\section{FIELD STRATEGIES AND LIMITATIONS}

In general, field studies of natural biodegradation at actual sites have used a one dimensional approach: changes in organic and inorganic chemistry along the groundwater flow path are related to background conditions in order to evaluate plume biodegradation. One of the strengths of these types of studies is that they represent the complexity of hydrogeologic and contaminant conditions that are typical of industrial contamination. However, this complexity also imposes a limitation. Since three dimensional characterization of the source and plume is not usually possible, there is limited ability to formulate interpretations based on mass balance and distribution and an associated limitation on determining plume-scale rates of biodegradation.

Other natural biodegradation studies have used controlled field experiments to avoid problems associated with poorly-defined sources and plumes. This approach has typically involved injection of a measured slug of water containing known concentrations of organics. Barker et al. (1987) injected a slug containing benzene, toluene and xylenes (BTX) into the Borden aquifer. They monitored the resulting plume and were able to calculate mass balances to determine the 
persistence of the organics over time. In a similar study at Columbus Air Force Base, Macintyre et al. (1993) injected a slug containing four organics: two aromatics, a PAH and a chlorinated compound. Again, the authors were able to use a mass balance approach to determine plumescale decay rates.

Controlled field studies based on slug-type plumes have an advantage over those using actual groundwater contamination occurrences, because the researchers know the precise mass and composition of the organic substrate and the time period over which it was introduced. Also, since the approximate migration path of the plume is known in advance, plume monitoring can be more focussed, and conducted in greater detail. However, a shortcoming of this approach is that it does not provide a realistic analog to actual contaminated sites. Since the source is short-lived, the resulting plume is transient, moving through the aquifer as a slug until it disappears completely. Consequently, the plume will not display aspects of long term behaviour, such as an approach to steady state, which may be of particular interest for plumes originating from fixed sources.

\section{STUDY APPROACH AND PURPOSE}

The focus of the current research is a source of creosote emplaced below the water table at CFB Borden in August, 1991. Since the time of emplacement, a complex plume of dissolved organics has been forming under natural gradient conditions, and detailed monitoring has been conducted to determine the extent of mass loss. The overall purpose of this research was to contribute to the understanding of natural attenuation as it relates to complex hydrocarbon plumes. The controlled field experiment described herein represents a unique setting and approach for this type of research. It is based on an emplaced source and should therefore provide a reasonable analog to actual contaminated sites. However, data have been collected at a level of detail that would typically not be feasible on an actual contaminated site. The relatively high density of data points allows a more conclusive evaluation of organic mass distribution and transformation, using a mass balance approach. The author knows of only one other study where a separate phase organic substance was emplaced below the water table as part of a controlled field experiment (Rivett, 1995). However, the emphasis of that research differs significantly from the current project in that it involved a relatively simple mixture of chlorinated hydrocarbons that was examined under conditions with limited potential for biodegradation. Creosote, the organic liquid used in this study, was selected because it is a complex mixture of biodegradable hydrocarbons, typical of many other organic mixtures.

Chapter 2 provides an overview of the research and also provides a qualitative description of plume development, based on dissolved phase monitoring. In Chapter 3, a more quantitative, mass balance approach is used to determine the occurrence and amount of organic compound transformation and in Chapter 4, multiple lines of evidence are used to evaluate whether the observed plume transformation is due to biodegradation. The conceptual model that is generally accepted for organic plume degradation is described in Chapter 5 , and some processes in the 
study plume are compared with the model to evaluate whether it is adequately descriptive.

Conclusions and recommendations related to the research are provided in Chapters 6 and 7 , respectively. 


\section{CHAPTER 2 PROJECT OVERVIEW}

\section{SITE DESCRIPTION}

The field research site is located at Canadian Forces Base (CFB) Borden, approximately $80 \mathrm{~km}$ northwest of Toronto, Ontario, Canada. It is in an unused sand pit situated approximately $350 \mathrm{~m}$ north of a landfill that operated from 1970 to 1976 . Several hydrogeological studies have been conducted at this location over the past 15 years and, consequently, many of the parameters affecting the fate and transport of solutes in groundwater have been evaluated in exceptional detail. A summary of groundwater and aquifer parameters is provided in Table 2.1.

The site is underlain by an unconsolidated sand aquifer consisting of medium- to fine-grained sand. These deposits are glaciolacustrine in origin and grade into silts and clays at a depth of approximately nine metres. MacFarlane et al. (1983) and Nicholson et al. (1983) discussed the inorganic chemistry and migration of a groundwater leachate plume originating from the landfill and underlying the sand pit study site at depths ranging from six to seven metres. From the water table to the top of the leachate plume, groundwater is largely unaffected by the landfill. The current experiment has been conducted within this unaffected zone.

Chemistry of background groundwater (unaffected by the leachate plume) at the site was examined by Nicholson et al. (1983) and Mackay et al. (1986) and also through the current study. A summary of results is provided in Table 2.2. The groundwater is relatively hard with low dissolved organic carbon. Dissolved oxygen was determined to be variable but in general the aquifer was aerobic with oxygen ranging up to $8.5 \mathrm{mg} / \mathrm{L}$. Data collected through this study indicate that the dissolved oxygen concentration in groundwater directly upgradient of the creosote source was 2 to $3 \mathrm{mg} / \mathrm{L}$. The landfill leachate plume located is characterized by nondetectable dissolved oxygen (Barbaro et al., 1992) as well as major ion concentrations that are generally at least an order of magnitude greater than those that occur in background groundwater (Nicholson et al., 1983).

\section{CREOSOTE COMPOSITION AND PROPERTIES}

Creosote is used as an industrial wood preservative and is a thin oily liquid. Since it has a density slightly greater than that of water and is composed of hydrophobic compounds it is classified as a dense nonaqueous phase liquid (DNAPL). Creosote may consist of up to 200 chemicals (Mueller et.al., 1989): approximately $85 \%$ PAHs, $10 \%$ phenolic compounds and $5 \%$ oxygen-, sulphur-, and nitrogen-heteroaromatic compounds. The range of the component solubilities varies by several orders of magnitude. 
Table 2.1. Characteristics of the Borden aquifer

\begin{tabular}{|c|c|c|}
\hline Parameter & Method/Result & Source \\
\hline Mineralogy & $\begin{array}{l}\text { analysis of bulk sample by X-ray diffraction: quartz } \\
58 \% \text {, feldspars } 19 \% \text {, carbonates } 14 \% \text {, amphiboles } \\
7 \% \text {, chlorite } 2 \%\end{array}$ & Mackay et al. (1986) \\
\hline Porosity & $\begin{array}{l}\text { volume-weighted arithmetic mean of } 36 \text { samples: } \\
0.33\end{array}$ & Mackay et al. (1986) \\
\hline Bulk density & $\begin{array}{l}\text { volume-weighted arithmetic mean of } 36 \text { samples: } \\
1.81 \mathrm{~g} / \mathrm{cm}^{3}\end{array}$ & Mackay et al. (1986) \\
\hline $\begin{array}{l}\text { Solids } \\
\text { density }\end{array}$ & $\begin{array}{l}\text { volume-weighted arithmetic mean of } 36 \text { samples: } \\
2.71 \mathrm{~g} / \mathrm{cm}^{3}\end{array}$ & Mackay et al. (1986) \\
\hline $\begin{array}{l}\text { Organic } \\
\text { carbon } \\
\text { content }\end{array}$ & average of $0.02 \%$; ranging from $0.01 \%$ to $0.09 \%$ & Mackay et al. (1986) \\
\hline $\begin{array}{l}\text { Hydraulic } \\
\text { conductivity }\end{array}$ & $\begin{array}{l}\text { - slug tests at } 26 \text { points; mean of } 7 \times 10^{-5} \mathrm{~m} / \mathrm{s} \\
\text { - falling head permeameter tests with } 1279 \text { samples; } \\
\text { overall geometric mean of } 9.75 \times 10^{-5} \mathrm{~m} / \mathrm{s}\end{array}$ & $\begin{array}{l}\text { Mackay et al. (1986) } \\
\text { Sudicky (1986) }\end{array}$ \\
\hline $\begin{array}{l}\text { Depth to } \\
\text { water table }\end{array}$ & $\begin{array}{l}\text { Varies with time from ground surface to } 1.5 \mathrm{~m} \\
\text { below grade }\end{array}$ & $\begin{array}{l}\text { Linderfelt and Wilson } \\
\text { (1994) }\end{array}$ \\
\hline $\begin{array}{l}\text { Hydraulic } \\
\text { gradient }\end{array}$ & $\begin{array}{l}\text { ranged from } 0.002 \text { to } 0.0053 \text { over first two years of } \\
\text { current study period; time-weighted average of } \\
0.0039\end{array}$ & Linderfelt (pers. comm.) \\
\hline $\begin{array}{l}\text { Ground } \\
\text { water flow } \\
\text { direction }\end{array}$ & $\begin{array}{l}\text { ranged from } \mathrm{N} 11^{\circ} \mathrm{W} \text { to } \mathrm{N} 50^{\circ} \mathrm{E} \text { over first two years of } \\
\text { current study period; predominant flow direction } \\
\text { was } \mathrm{N} 21^{\circ} \mathrm{E}\end{array}$ & Linderfelt (pers. comm.) \\
\hline $\begin{array}{l}\text { Ground } \\
\text { water } \\
\text { velocity }\end{array}$ & $\begin{array}{l}\text { - } 1038 \text { day tracer test: } 0.091 \mathrm{~m} / \text { day } \\
\text { - } 476 \text { day tracer test: } 0.0933 \text { to } 0.0947 \mathrm{~m} / \text { day } \\
\text { - } 108 \text { day tracer test: } 0.081 \mathrm{~m} / \text { day } \\
\text { - emplaced source monitoring: } 0.066 \text { to } 0.085 \mathrm{~m} / \text { day }\end{array}$ & $\begin{array}{l}\text { Mackay et al. (1986) } \\
\text { Hubbard et al. (1994) } \\
\text { Patrick (1986) } \\
\text { this study }\end{array}$ \\
\hline $\begin{array}{l}\text { Apparent } \\
\text { dispersivity }\end{array}$ & 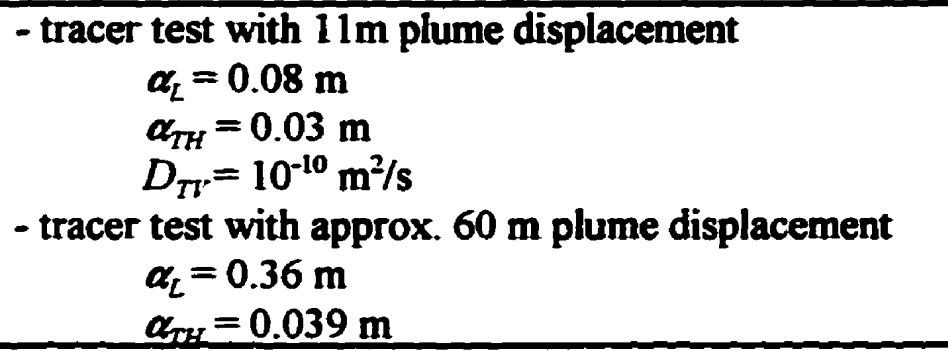 & $\begin{array}{l}\text { Sudicky et al. (1983) } \\
\text { Freyberg (1986) }\end{array}$ \\
\hline
\end{tabular}


The raw creosote used in this study was amended with certain compounds so it would be representative of typical composition (Mueller, 1989). To $69.5 \mathrm{~kg}$ of creosote the following compounds were added: $0.45 \mathrm{~kg}$ carbazole, $0.50 \mathrm{~kg}$ p-cresol, $1 \mathrm{~kg}$ phenol. M-xylene was also added $(3 \mathrm{~kg})$, to provide a compound representative of petroleum hydrocarbon contamination. The density of the modified creosote was $1.03 \mathrm{~g} / \mathrm{mL}$.

Table 2.2. Background groundwater characteristics

\begin{tabular}{lcc}
\hline Parameter & Range & Source \\
\hline Calcium & $50-100 \mathrm{mg} / \mathrm{L}$ & 1,2 \\
Magnesium & $2.4-6.1 \mathrm{mg} / \mathrm{L}$ & 1,2 \\
Sodium & $0.9-2.0 \mathrm{mg} / \mathrm{L}$ & 1,2 \\
Potassium & $0.1-1.2 \mathrm{mg} / \mathrm{L}$ & 1,2 \\
Alkalinity (as $\left.\mathrm{CaCO}_{3}\right)$ & $100-250 \mathrm{mg} / \mathrm{L}$ & 1 \\
Chloride & $1-3 \mathrm{mg} / \mathrm{L}$ & $1,2,3$ \\
Sulphate & $10-30 \mathrm{mg} / \mathrm{L}$ & 1,3 \\
Nitrate & $<0.6-6 \mathrm{mg} / \mathrm{L}$ & $1,2,3$ \\
TDS & $380-500 \mathrm{mg} / \mathrm{L}$ & 1 \\
DOC & $<0.7 \mathrm{mg} / \mathrm{L}$ & 2 \\
DO & $0-8.5 \mathrm{mg} / \mathrm{L}$ & 2,3 \\
Temperature & $6-15{ }^{\circ} \mathrm{C}$ & $1,2,3$ \\
pH & $7.1-7.9$ & $1,2,3$ \\
\hline
\end{tabular}

Sources:

1 - Nicholson et al. (1983)

2 - Mackay et al. (1986)

3 - this study

\section{METHODS}

\section{Source Emplacement}

The creosote source was emplaced on August 28, 1991, and is shown schematically in Figure 2.1. To install the source, sealable sheet piling (Starr et al., 1991) was vibrated into the ground in a rectangular array $(5 \times 1.5 \mathrm{~m})$. The sand inside the sheet piling was excavated after it was dewatered with a shallow dewatering well. The source sand was mixed with creosote in a cement mixer at less than 5\% creosote by volume: laboratory testing had indicated that it should be effectively immobile at this concentration. A total of approximately $74 \mathrm{~kg}$ of creosote was added to approximately $5800 \mathrm{~kg}$ of sand. Several $\mathrm{kg}$ of $\mathrm{NaCl}$ was also mixed into the source sand, to provide a conservative tracer during source dissolution and plume development. 

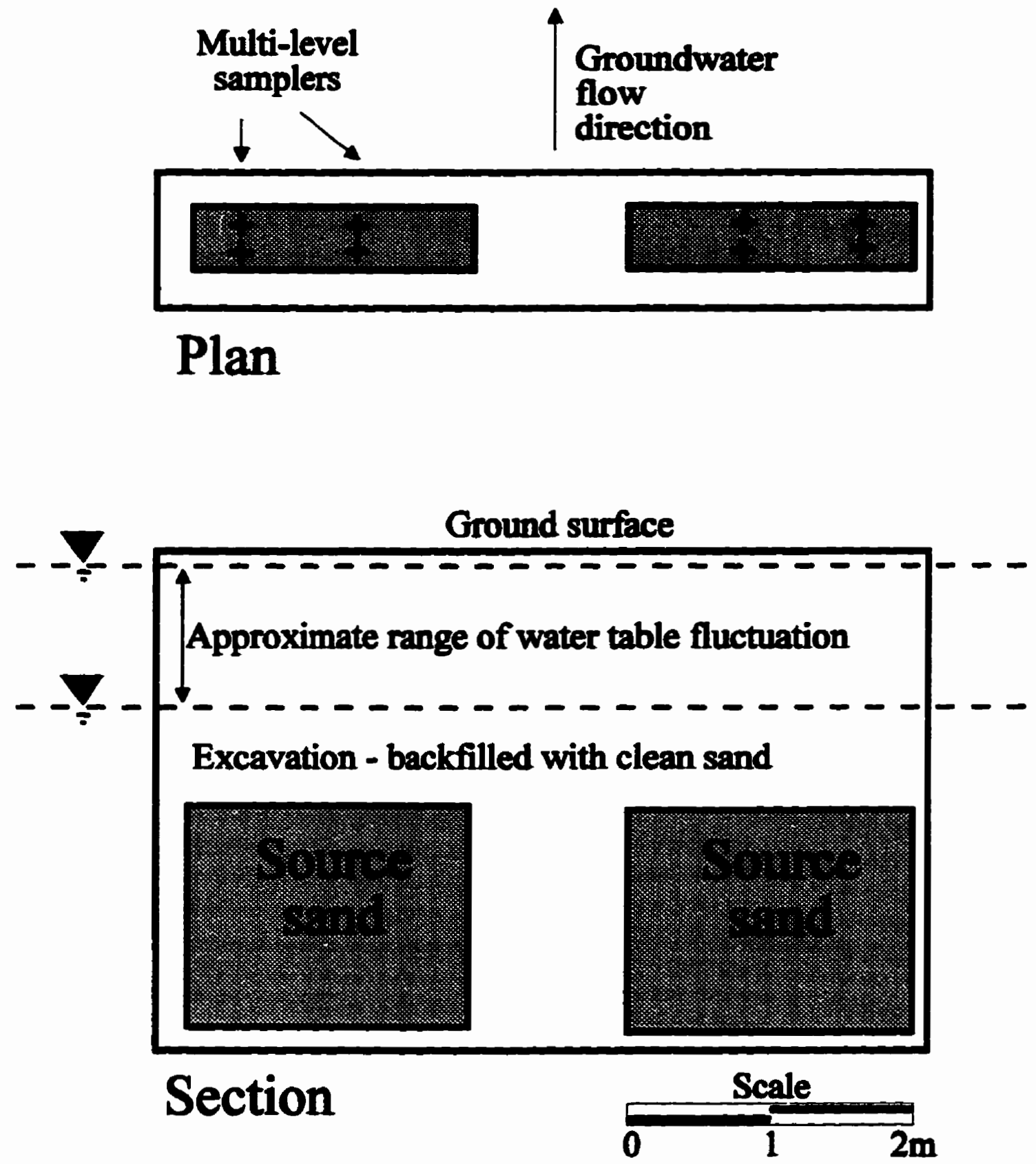

Figure 2.1. Configuration of creosote source: (top) plan view showing location of multilevel samplers installed through source and (bottom) view of section perpendicular to the direction of groundwater flow. 
Borden sand was not used for the source material since the addition of creosote to the sand would result in some decrease in porosity that could inhibit the movement of groundwater through the source. Instead a coarser sand was used, with a hydraulic conductivity of approximately $3.6 \mathrm{X}$ $10^{-4} \mathrm{~m} / \mathrm{s}$, based on sieve analyses (Devlin, 1994) - approximately 5 times greater than Borden sand. Column tests by Forsey (in progress) showed that addition of $5 \%$ creosote by volume to the source sand would results in a hydraulic conductivity reduction of approximately $9.7 \%$. Since the resulting source hydraulic conductivity is considerably greater than ambient Borden conditions, groundwater flow through the source should not be inhibited. Evaluation of groundwater flux through the source and flux of creosote compounds out of the source is provided in the Chapter 3. Additional details of source installation are provided by Malcolmson (1992).

\section{Plume Monitoring Network}

The creosote source was emplaced hydraulically upgradient of an existing network of multi-level samplers, shown in Figure 2.2. The network was originally constructed for a plume study conducted in the early 1980's (Mackay et al., 1986). Since then, it has been augmented and modified by three subsequent studies (Patrick, 1986; Hubbard et al., 1994; Berry-Spark et al., 1988). A tabular summary of piezometers installed prior to this study was provided by Hubbard et al. (1994). In addition, 12 bundle piezometers were added along the west side of the system for the current study, and specifications of these are provided in Appendix 1. A total of 257 of the available 380 network multi-level samplers were monitored for this study. The horizontal spacing of the samplers is closer near the source (down to approximately one metre) and increases to approximately four metres at the downgradient extent of the system.

Figure 2.2 also shows a profile of the monitoring points along a cross-section (A-A') that approximates the plume centre line. The samplers are typically constructed with 14 sampling levels with a vertical spacing of 20 or, less frequently, $30 \mathrm{~cm}$. This spacing was intended to approximate the vertical correlation scale estimated for hydraulic conductivity of the Borden aquifer (Sudicky, 1986). The samplers typically span a depth range of approximately 1.8 to 4.4 $\mathrm{m}$ below grade, with maximum depth increasing to $5.5 \mathrm{~m}$ at the downgradient end of the system.

Sampler construction was based on a design developed in earlier studies at the Borden site (Cherry et al., 1983), and similar methods were used for installation. In the "near-source" zone of the plume $(x \leq 35 \mathrm{~m})$ samplers are constructed of $3.2 \mathrm{~mm}$ O.D. flexible teflon tubing, to minimize sorption of hydrophobic organics to sampling materials. Within the monitoring period of this study only chloride, phenol and naphthalene showed appreciable solute migration beyond this zone. Outside this zone, most samplers are constructed with $3.2 \mathrm{~mm}$ O.D. polyethylene tubing. 


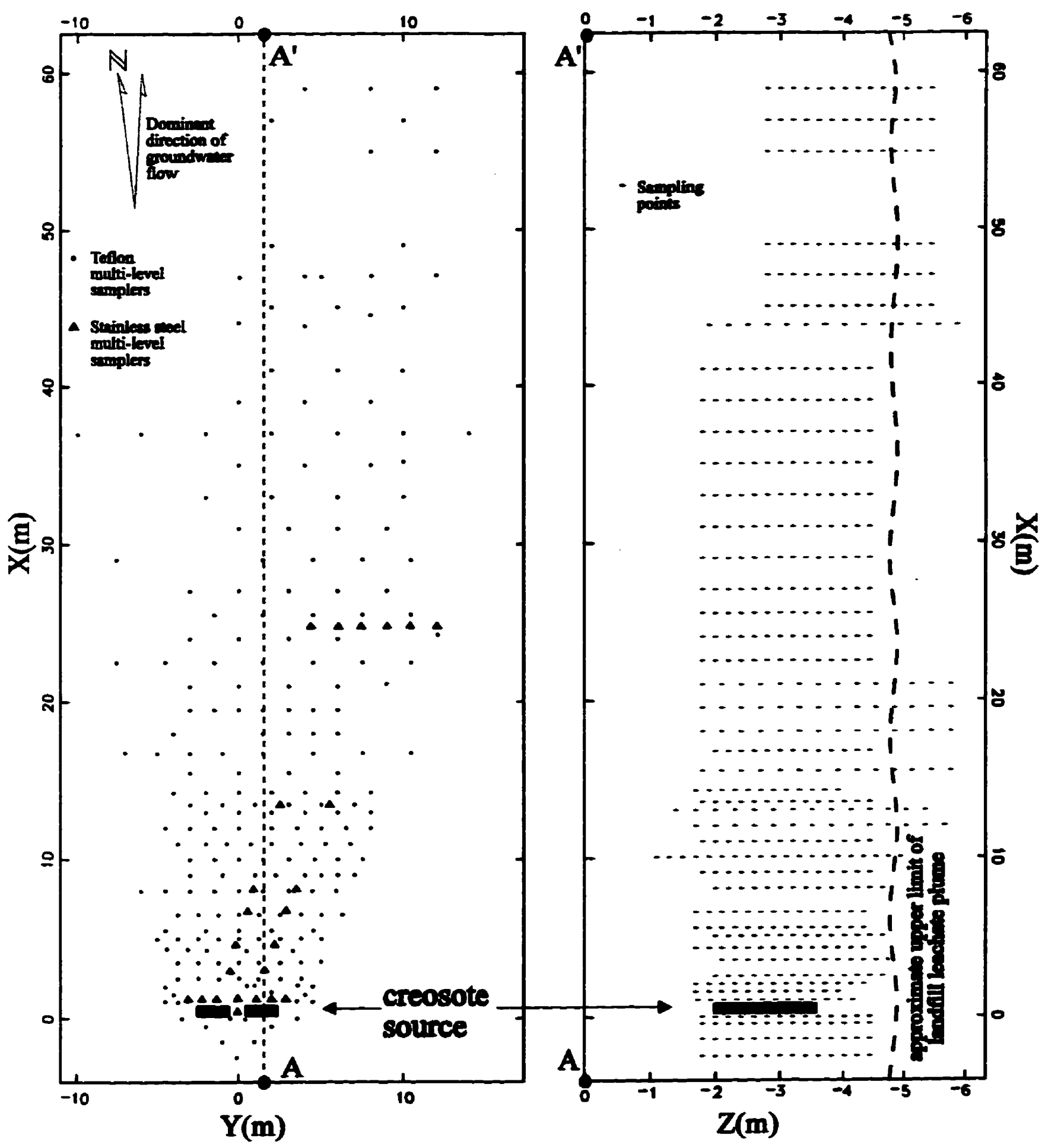

Figure 2.2. Location of the creosote source and plume monitoring network: (left) plan view of multi-level samplers used for synoptic sampling and (right) approximate vertical distribution of sampling points along, or adjacent to, section AA'. 


\section{Plume Sampling}

Synoptic plume sampling was conducted six times after source emplacement. The purpose of this type of sampling was to provide detailed, three dimensional data for determining changes in plume distribution and mass with time. A summary of the synoptic monitoring program is provided in Table 2.3. In total, more than 6000 samples were analyzed for the six synoptic monitoring events. These data are provided in Appendix 2, which is provided on computer disk due to the large number of data points.

In addition, approximately 20 sampling points, located throughout the plume, were monitored approximately monthly over the four year duration of this study and data are provided in Appendix 3, also on computer disk. In part, the standard teflon multi-level samplers were used for this "time series" monitoring. However, the monitoring system was also augmented with a series of $\mathbf{4 0}$ additional multi-level samplers for this purpose. These samplers were constructed with $3.2 \mathrm{~mm}$ O.D. stainless steel tubing, for the purpose of minimizing sorption of hydrophobic organics. This was considered especially important since some of these samplers were actually installed within and just outside the creosote source. Each of these piezometers consisted of from seven to 13 monitoring points, again separated by a vertical distance of 20 to $30 \mathrm{~cm}$. Locations are indicated on Figures 2.1 and 2.2; specifications are provided by Nielsen and Hansen (1992).

Table 2.3. Summary of synoptic monitoring program

\begin{tabular}{lcc}
\hline Date & Days after source emplacement & Number of samples analyzed \\
\hline Oct. $22-23,1992$ & 55 & 450 \\
June $1-3,1992$ & 278 & 755 \\
Nov. $9-10,1992$ & 439 & 564 \\
May $11-21,1993$ & 626 & 2809 \\
May 30-June 3, 1994 & 1008 & 1634 \\
May 15-19, 1995 & 1357 & 1632 \\
\hline
\end{tabular}

Synoptic samples were collected in $18 \mathrm{~mL}$ glass hypovials and capped with crimped aluminum caps lined with teflon-coated silica septa. A vacuum manifold system constructed to collect 14 samples simultaneously, and identical to that used in earlier studies at the site (Mackay et al. 1986; Hubbard et al. 1994), was used for sample collection. In collecting samples, all the sampling tubes associated with a given multi-level sampler were connected to the manifold and a vacuum was applied to the system to draw groundwater into the sample bottles. At least $50 \mathrm{~mL}$ of groundwater was pumped through the system and the sample bottles, for purging before sample collection. After collection, samples were capped as rapidly as possible to minimize volatilization. They were then placed in coolers and transported to the University of Waterloo for analysis. A similar procedure was used for collection of time series samples except that $45 \mathrm{~mL}$ glass hypovials and a single-tube vacuum system were used. 
Sample Analysis

The analytical methodology used for the study was developed for quantification of creosote compounds in groundwater, sand and pure creosote. Compounds selected for analysis were intended to be representative of the main groups of compounds found in creosote and included two phenolic compounds (phenol and 2,6-dimethylphenol), five PAHs (naphthalene, 1methylnaphthalene, anthracene, pyrene and phenanthrene) and two heteroaromatic compounds (carbazole and dibenzofuran). M-xylene was included in the analytical suite as a representative petroleum hydrocarbon. Analysis was also conducted for several other compounds but due to the variable availability of analytical standards, only the 10 compounds above were analyzed throughout the whole experimental period.

Groundwater samples (based on $45 \mathrm{~mL}$ ) were prepared by adding $9 \mathrm{~g} \mathrm{NaCl}$ to promote solvent partitioning of all analytes and $1 \mathrm{~mL} 1 \mathrm{~N} \mathrm{HCl}$ to enhance partitioning of phenolic compounds. Samples were then capped and allowed to stand for 24 hours before addition of $3 \mathrm{~mL}$ solvent (dichloromethane). Samples were then placed on a shaker for 20 minutes at 350-400 rpm and 1 $\mathrm{mL}$ of the solvent was removed to an autosampler vial. Pure creosote was prepared for analysis by dissolving a $10 \mathrm{uL}$ sample in $10 \mathrm{~mL}$ dichloromethane and then transferring $1 \mathrm{~mL}$ to an autosampler vial.

Extracts were injected into a Hewlett Packard 5890 Gas Chromatograph (GC) equipped with an HP7673A autosampler and Flame Ionization Detector (FID). The temperature program for analysis was as follows: $40{ }^{\circ} \mathrm{C}$ for $0.5 \mathrm{~min}, 15^{\circ} \mathrm{C} / \mathrm{min}$ to $300^{\circ} \mathrm{C}$, hold $10 \mathrm{~min}$. The carrier gas was helium with a flow rate of approximately $24 \mathrm{~mL} / \mathrm{min}$. The injector temperature from the $\mathrm{GC}$ was $275^{\circ} \mathrm{C}$, FID temperature was $325^{\circ} \mathrm{C}$ and injection volume was 3uL. Additional details on the analytical methodology are provided in Appendix 4.

Source Composition

Quantitative analytical results (according to the analytical method described above) for ten organic compounds in the creosote used for this study are shown in Table 2.4 and original data are provided by Malcolmson (1992). As indicated, these ten compounds comprise $39 \%$ of the mixture. Identification of many of the remaining compounds was conducted on a qualitative basis using Gas Chromatography/Mass Spectrometry (GC/MS) analysis. Creosote samples were extracted by three different methods in order to identify a wide range of compounds, with major components presented in Table 2.5. A "hydrocarbon" fraction, extracted with toluene was dominated by PAHs with occurrence of an oxygen heteroaromatic (dibenzofuran). Extraction with methanol yielded a "resin" fraction that was dominated by phenois, substituted phenols and nitrogen heteroaromatics (e.g., isoquinoline and benzocarbazole). A third extraction was performed by treating a creosote sample with a dilute solution of $\mathrm{NaOH}$, followed by extraction to dichloromethane. The residual aqueous layer was then acidified and extracted again with dichloromethane to yield an "acid" fraction that was dominated by phenol, substituted phenols and oxygen heteroaromatics. Additional details on this analysis are provided in Appendix 5. 
Table 2.4. Quantitative analysis of creosote and compound properties

\begin{tabular}{|c|c|c|c|c|c|c|c|}
\hline \multirow[t]{2}{*}{ Compound } & \multirow{2}{*}{$\begin{array}{c}\text { Concentration }{ }^{\mathrm{l}} \\
\text { (mg/kg } \\
\text { creosote) } \\
(n=8)\end{array}$} & \multirow[t]{2}{*}{$\log K_{\text {orr }}{ }^{2}$} & \multirow[t]{2}{*}{$\begin{array}{l}\text { Melting } \\
\text { Point } \\
\text { (deg C) }\end{array}$} & \multirow[t]{2}{*}{$\begin{array}{c}\text { Solid } \\
\text { Solubility } \\
(\mathrm{mg} / \mathrm{L})\end{array}$} & \multirow[t]{2}{*}{$\begin{array}{l}\text { Liquid } \\
\text { Solubility" } \\
(\mathrm{mg} / \mathrm{L})\end{array}$} & \multicolumn{2}{|c|}{$\begin{array}{l}\text { Estimate of Initial } \\
\text { Aqueous Conc. }(m \circ / \mathrm{L})\end{array}$} \\
\hline & & & & & & Raoult's Law" & Batch Test \\
\hline$m$-xylene & 38504 & 3.12 & -47.4 & - & $196^{6}$ & 11.0 & 14.4 \\
\hline phenol & 14064 & 1.50 & 43.0 & 82000 & 158775 & 840 & 1388 \\
\hline 2,6-dimethylphenol & 194 & 2.26 & 49.0 & 6150 & 13719 & 2.5 & not detected \\
\hline naphthalene & 96061 & 3.35 & 81.0 & 31.7 & 150.5 & 17 & 12.4 \\
\hline phenanthrene & 123703 & 4.52 & 101 & 1.3 & 9.9 & 1.02 & 0.44 \\
\hline anthracene & 12338 & 4.50 & 216 & 0.075 & 8.61 & 0.0831 & not detected \\
\hline dibenzofuran & 43743 & 4.12 & 86.5 & 10.03 & 54.2 & 2.1 & 1.6 \\
\hline carbazole & 3276 & 3.72 & 247 & 1.03 & 245.8 & 0.698 & 0.23 \\
\hline pytene & 39141 & 5.00 & 156 & 0.14 & 3.9 & 0.113 & 0.03 \\
\hline 1-methvlnaphthalene & 19127 & 3.87 & -22 & $=$ & $28.5^{6}$ & 0.572 & 1.7 \\
\hline Fraction of total & & & & & & & \\
\hline mass quantified & 0.39 & & & & & & \\
\hline
\end{tabular}

\footnotetext{
Analyses performed after amendment with additional compounds ( $m$-xylene, carbazole, $p$-cresol, and phenol). From Sangster, 1989.

From Mackay et al., 1992.

Calculated with Equation (2.2) using solid solubility, melting temperature and system temperature of $15^{\circ} \mathrm{C}$.

Calculated with Equation (2.1)

Liquid solubility used directly since compounds are liquid at system temperature.
}

\section{Source Dissolution and Leachate Composition}

An extension of Raoult's law to partitioning between an aqueous phase and an organic mixture can be used to generate a qualitative prediction of how the composition of source leachate might be expected to change over time. According to this approach, components in the mixture are said to dispiay ideal behaviour if they partition linearly to the aqueous phase in proportion to the product of their mole fraction in the organic phase and their pure compound solubility. Mackay et al. (1991) pointed out two assumptions inherent in this approach: 1) the activity coefficient in the organic phase is equal to unity - a reasonable approximation for organic mixtures composed of compounds with similar chemical properties and 2) the inverse of the activity coefficient for the solute in water is equal to the mole fraction solubility of the component. Shiu et al. (1988) incorporated these assumptions when they stated the concentration form of Raoult's law as:

$C^{\prime}=x^{s} S_{L}^{i}$

where $C^{l}$ is the aqueous concentration of compound $i, x^{\prime}$ is the mole fraction of $i$ in the organic 
Table 2.5. Qualitative analysis of creosote composition

"Hydrocarbon" fraction"

\begin{tabular}{|c|c|c|c|}
\hline Compound & $\begin{array}{l}\text { Relative } \\
\text { conc. }\end{array}$ & Compound & $\begin{array}{l}\text { Relative } \\
\text { conc. }\end{array}$ \\
\hline $\begin{array}{l}\text { indene } \\
\text { naphthalene } \\
\text { 2-methyl naphthalene } \\
\text { 1-methyl naphthalene } \\
\text { acenaphthalene } \\
\text { biphenyl } \\
\text { dimethylnaphthalenes } \\
\text { dibenzofuran } \\
\text { fluorene } \\
\text { phenanthrene } \\
\text { anthracene } \\
\text { methyl phenanthrenes }\end{array}$ & $\begin{array}{l}+ \\
+++ \\
+H \\
+ \\
+ \\
+ \\
+ \\
+ \\
+H \\
+ \\
+\end{array}$ & $\begin{array}{l}\text { cyclopenta phenanthrene } \\
\text { fluoranthene } \\
\text { pyrene } \\
\text { benzo(a)fluoranthene } \\
\text { benzophenanthrene } \\
\text { chrysene } \\
\text { benzofluoranthene(b) } \\
\text { benzofluoranthene(E) } \\
\text { benzo(E)pyrene } \\
\text { benzo(A)pyrene } \\
\text { perylene } \\
\text { benzperylenes }\end{array}$ & $\begin{array}{l}+ \\
++ \\
++ \\
+ \\
+ \\
++ \\
+ \\
+ \\
+ \\
+ \\
+ \\
+\end{array}$ \\
\hline \multicolumn{4}{|l|}{ "Resin" fraction" } \\
\hline Compound & $\begin{array}{l}\text { Relative } \\
\text { conc. }\end{array}$ & Compound & $\begin{array}{l}\text { Relative } \\
\text { conc. }\end{array}$ \\
\hline $\begin{array}{l}\text { phenol } \\
\text { methyl phenols } \\
\text { dimethyl phenols } \\
\text { ethyl,methyl phenols } \\
\text { isoquinoline } \\
\text { benzene acetonitrile } \\
\text { methylquinolines } \\
\text { dimethylquinolines }\end{array}$ & $\begin{array}{l}+4 \\
+H+ \\
++ \\
+ \\
H+ \\
++ \\
+\end{array}$ & $\begin{array}{l}\text { methyl indole } \\
\text { carbazole } \\
\text { benzoquinolines } \\
\text { nitrosocarbazole(?) } \\
\text { anthracene carbonitrile } \\
\text { fluorene carbonitrile } \\
\text { fluorantheneamine } \\
\text { benzocarbazoles }\end{array}$ & $\begin{array}{l}+ \\
+ \\
+1+ \\
+++ \\
+ \\
+ \\
+ \\
+\end{array}$ \\
\hline
\end{tabular}

"Acid" fraction ${ }^{1}$

\begin{tabular}{llll}
\hline Compound & $\begin{array}{l}\text { Relative } \\
\text { conc. }\end{array}$ & Compound & $\begin{array}{l}\text { Relative } \\
\text { conc. }\end{array}$ \\
\hline phenol & + & methyl naphthalenols & +++ \\
2-methyl phenol & ++ & biphenyl-ols & ++ \\
4-methyl phenol & +++ & dibenzofuranols & ++ \\
dimethyl phenols & +++ & phenanthrenols & ++ \\
ethyl, methyl phenol & + & benzonaphthofurans & ++ \\
naphthalenols or phenyl furans & +++ & & \\
\hline
\end{tabular}

See text for description of analytical methodology. 
mixture and $S_{L}{ }^{t}$ is the pure compound liquid solubility. For compounds that are solid in pure form and at the temperature of the given system, "super-cooled" liquid phase solubility can be calculated with the following (Shiu et al., 1988):

$S_{L}{ }^{\prime}=S_{s}^{i} \exp \left[6.8\left(T_{M^{\prime}} / T-1\right)\right]$

where $S_{S}{ }^{\prime}$ is solid solubility, $T_{M}{ }^{i}$ is melting point $\left({ }^{\circ} \mathrm{K}\right)$ and $T$ is the system temperature. The calculated liquid solubility will be greater than the solid solubility but, as pointed out by Mackay et al. (1991), the dissolved concentration can never exceed the latter.

Several studies have examined the degree to which various compositionally complex organic mixtures conform the Raoult's law model of ideal behaviour. Cline et al. (1991) determined, through batch-type testing, that a variety of 30 gasolines conformed closely to Raoult's law. Aqueous partitioning of eight PAHs from four different diesel fuels was investigated with batch tests by Lee et al. (1992a) and determined to be well described (within a factor of two) by assuming ideal behaviour. A similar degree of agreement was reported by Lee et al. (1992b) for partitioning of PAHs from eight different coal tar samples, also with batch tests. Priddle and MacQuarrie (1994) examined the solubility of creosote with a dynamic column experiment. In a mass transfer rate test, five of seven PAHs conformed to ideal behaviour within a factor of two.

In order to evaluate the applicability of the ideal behaviour assumption to the creosote used in this study, initial source leachate concentrations calculated with Raoult's law were compared with concentrations predicted from laboratory batch dissolution tests, and results are shown in Table 2.4. Calculation of the former was done with Equation (2.1) using pure compound liquid solubilities directly from the literature or calculated on the basis of literature values for $S_{S}{ }^{i}$ and $T_{M f}{ }^{t}$. In order to estimate mole fractions $(x)$ for the ten quantified compounds, an average molecular weight of 150 was assumed for the unquantified portion of the creosote $(61 \%)$ on the basis of qualitative comparison between $G C$ retention times for identified and unidentified compounds. Methodology and results for the batch test were originally presented by Malcolmson (1992) and are summarized by King et al. (1994), attached as Appendix 6. Comparison of the two sets of results indicates that Raoult's law may provide a useful approximation of initial creosote dissolution, with concentrations of most compounds corresponding within a factor of four. Near source plume data, presented by King et al. (1994) (Appendix 6), indicated that initial concentrations in leachate from the emplaced source also showed reasonable agreement with the Raoult's law prediction. 
A model based on a further application of Raoult's law was used to evaluate expected changes in source leachate composition over time. To do this, it was necessary to account for the removal of compound mass from the immobile NAPL phase by a mobile aqueous phase (groundwater) and to calculate the resulting changes in NAPL phase mole fractions. The leachate concentration $\left(C_{l}{ }^{\prime}\right)$ at the end of a given time step can be approximated by:

$C_{l}^{t}=x_{l}^{\prime} S_{L}^{i}=\left(\left(M_{0}^{i}-q A \Delta t C_{0}^{i}\right) / M_{0}{ }^{\top}\right) S_{L}^{i}$

where the subscripts 0 and $I$ denote $t=0$ and $t=I$, respectively, $C$ in this case is in units of moles $L^{3}, M$ is moles of $i$ in the NAPL phase, $q$ is Darcy flux through the source $(L T) ; A$ is the cross-sectional area of the source perpendicular to the direction of groundwater flow $\left(L^{3}\right)$ and $M^{T}$ =total moles of all compounds in the NAPL phase. The result of applying this model to the emplaced creosote source is shown in Figure 2.3. An initial value for $C_{0}{ }^{i}$ was obtained from the Raoult's law estimate of initial leachate concentration. Further, it was again assumed that the average molecular weight of the unidentified compounds ( $61 \%$ by weight) was 150 , that these compounds were insoluble and that dissolution of the identified compounds reached equilibrium in all groundwater flowing through the source. The estimate of average molecular weight used herein (150) differs from that used in 1994 paper (200) due to re-evaluation and improvement of this parameter estimate. This change has a relatively minor effect on predicted concentrations over the time span shown in Figure 2.3. A groundwater flux $(q)$ of $0.03 \mathrm{~m} /$ day has been used, based on the determination of ambient groundwater velocity $(0.091 \mathrm{~m} /$ day) and porosity $(0.33)$ by Mackay et al. (1986). A relatively short time step of 0.25 days was used up to 100 days, to allow for the relatively rapid depletion of phenolic compounds. Subsequently, the time step was increased to one day.

A similar approach was used by King et al. (1994) and was determined to produce reasonable agreement with actual source leachate at some monitoring locations. However, the actual data displayed a high degree of variability, which was attributed to heterogeneous distribution of creosote residual in the source. Nevertheless, the modelling results shown in Figure 2.3 were considered to provide a reasonable framework for qualitative prediction of trends in source leachate composition. As shown, concentrations of phenol and 2,6-dimethylphenol in source leachate were expected to decrease relatively rapidly, causing a small initial increase in concentrations of the other eight compounds, to slightly greater than $C / C_{0}=1 . M$-xylene, carbazole and naphthalene concentrations are expected to decrease significantly over the study period but to remain detectable. Meanwhile, concentrations of 1 -methylnaphthalene, phenanthrene, anthracene, dibenzofuran and pyrene were expected to remain relatively consistent.

\section{Potential Sources of Error}

A series of field and laboratory QAQC samples and experiments were conducted to evaluate: 1) possible systematic error in field and laboratory methodology for introduction of systematic ertor, 2) methods precision and 3) variability of the sampled media (groundwater with organic solute). Results are summarized below and details are provided in Appendix 7. 


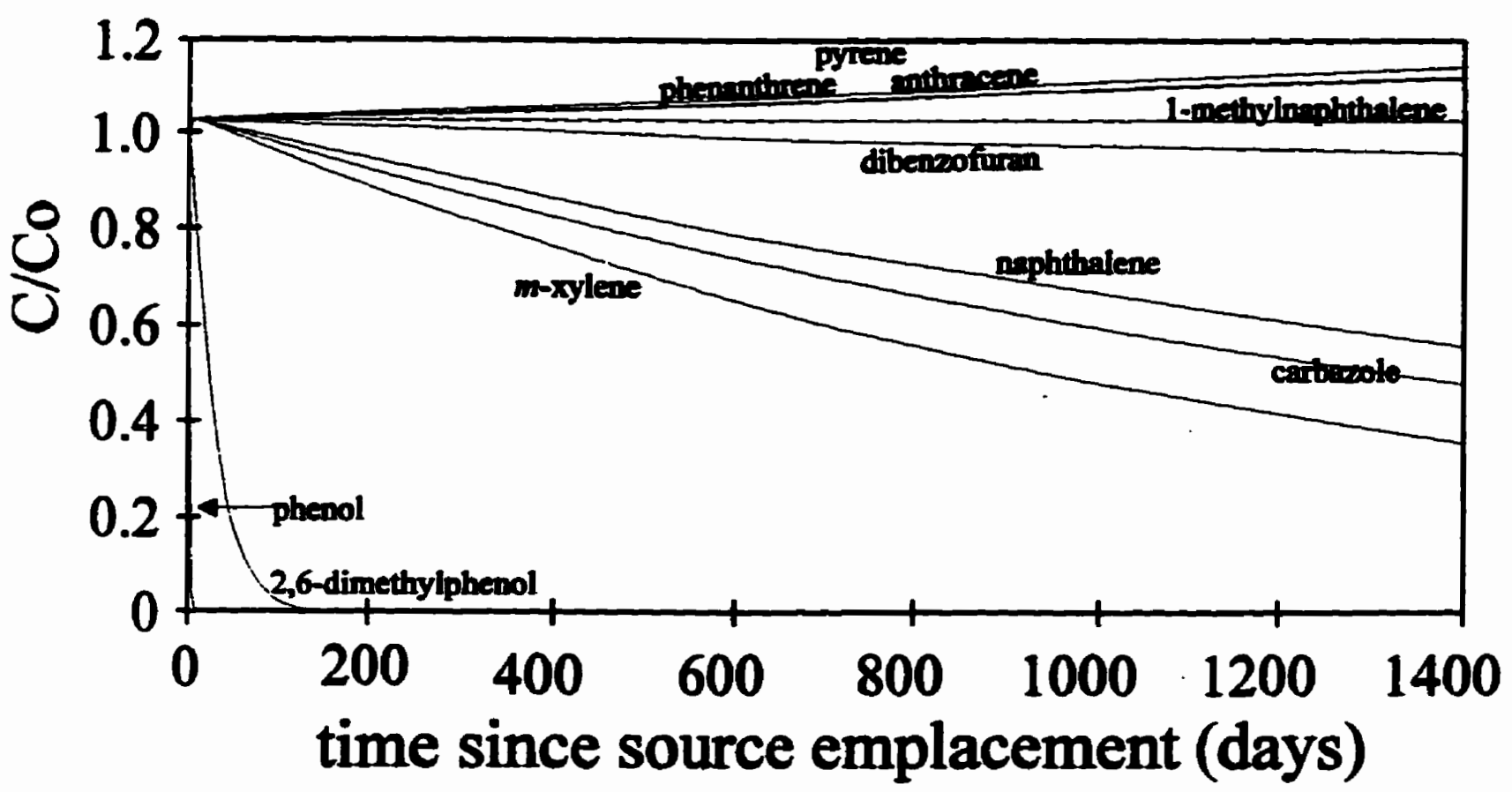

Figure 2.3. Aqueous concentration of selected creosote compounds in groundwater leaving the emplaced source; predicted according to model based on Raoult's law (see text for model assumptions). 
In general, the analytical procedure imparted a positive bias to measurements in the upper end of the concentration range and a negative bias in the lower end of the range. The total range of these biases was $+1-10 \%$. However, phenol results showed a larger and consistently negative bias in the range of $50 \%$, indicative of poor recovery for this compound. Correction of groundwater results for phenol recovery was not performed because the initial source mass of phenol was well known (a measured quantity was added to the creosote) and phenol disappeared to trace mass relatively rapidly, as discussed qualitatively later in this chapter and quantitatively in Chapter 3. Analytical results for other compounds were not corrected for percent recovery since they were generally within acceptable ranges. Measurement precision, evaluated as percent standard error, did not display an apparent trend with respect to solute concentration.

Samples were extracted and analyzed as rapidly as possibly. However, due to the large number of samples associated with the synoptic monitoring, holding times for some unextracted samples and extracted samples were as long as one week and four months, respectively. The largest delays in extraction and analysis were experienced for the 62 day synoptic monitoring event, since it incorporated the largest number of samples. An experiment to evaluate the effect of holding times over this range, for both water samples and sample extracts, indicated that systematic bias was not introduced. Through another experiment it was determined that sample concentrations were not significantly decreased by sorption to the teflon tubing in the multi-level samplers or the sampling manifold. Apparently, changes were minimized by the sampling procedure that included $50 \mathrm{~mL}$ purge prior to sample collection.

Evaluation of field blanks, composed of deionized water flushed through the manifold sampler after sample collection, indicated some potential for cross-contamination due to the manifold sampler. In a small portion $(<10 \%)$ of field blanks, there was evidence that naphthalene was introduced from the previous sample, although it was not noted for any other compounds. Where carry over of naphthalene occurred, concentrations in the blank were typically $1 \%$ of that in the previous sample. Results indicated that some minor, apparent "smearing" of the naphthalene plume may occur due to cross-contamination. The effect on naphthalene plume mass calculation should be much less than $1 \%$ since the carried over concentrations should be low, and should only be an issue at the periphery of the plume. Results from samples collected in series at the same monitoring points indicated that solute concentrations were not sensitive to the volume of groundwater recovered for sampling.

\section{RESULTS AND DISCUSSION}

\section{General}

The following is a qualitative description of plume development for chloride and six organic compounds: phenol, $m$-xylene, naphthalene, dibenzofuran, phenanthrene, carbazole and 1methylnaphthalene. Plume data are presented in three different formats to assist in visualization of plume behaviour. First, a series of horizontal plan-view diagrams are shown that depict contours based on the maximum concentration detected at each multilevel sampler (Figures 2.4 through 
2.8) for chioride and the first four organic compounds listed above. These contours have been produced with the GEOSOFT (Geosoft Inc., 1994) contouring package. Contours start at $x=2.7$ $\mathrm{m}$ from the source since steep concentration gradients at closer proximity to the source make it difficult to determine contour locations.

Figures 2.9 and 2.10 are plots of plume concentration versus distance from the source. These concentrations have been normalized to the cross-sectional area of the source in the plane approximately normal to the direction of groundwater flow $\left(6.8 \mathrm{~m}^{2}\right)$. This is defined as the $y-z$ plane, where the $z$ axis is vertical and the $y$ axis is horizontal and perpendicular to groundwater flow. The $x$-axis is orthogonal to the $y-z$ plane and is therefore approximately parallel to groundwater flow. The normalized concentrations shown in the figures were calculated through a three step procedure: 1) three dimensional plume data was interpolated to a regularly-spaced three dimensional grid, using the ENTEC (Surpac Inc., 1995) geostatistical package, 2) concentrations for each $\boldsymbol{x}$ step in the grid were then added to yield a concentration for each $\boldsymbol{x}$ value in the grid, 3) these values for concentration $(C)$ as a function of $x$, were then normalized to the cross-sectional area of the source by dividing the source cross-sectional area (in the $y-z$ plane) by that of a grid cell. Conceptually, this removes the effects of transverse horizontal and vertical dispersion from the plume data. Each of these curves is calculated using all of the dissolved concentration results for the given set of synoptic data. Inspection of the curves provides an indication of the longitudinal distribution of plume mass and the area under the curves indicative of the quantity of dissolved mass in the plume. Additional details on the gridding procedure are provided in Chapter 3. Time series data for two monitoring points within the plume $(x=6.7 \mathrm{~m}$, $y=0.55 \mathrm{~m}$ and $z=3.30 \mathrm{~m} ; x=24.85 \mathrm{~m}, y=4.45 \mathrm{~m}$ and $z=3.60 \mathrm{~m}$ ) are shown in Figure 2.11 .

\section{Chloride}

The $\mathrm{NaCl}$ added to the source during emplacement produced a plume of conservative tracer (chloride) that was used to evaluate average linear groundwater velocity. Velocity estimates determined through the current study were used primarily for comparison with earlier estimates to evaluate whether the latter were reasonable for current use. At 55 days, the chloride plume was showing signs of detaching from the source, indicating that the source $\mathrm{NaCl}$ was becoming depleted (Figure 2.4). By 439 days the chloride plume was completely detached and was migrating as a discrete slug. The longitudinal distribution of chloride concentration is shown in Figure 2.9 and, assuming that the maximum chloride concentration in source leachate occurred at early time, average linear groundwater velocity $(v)$ can be estimated according to:

$$
v=x \cdot t
$$

where $x=$ distance at which $C / C_{o}=0.5$ (estimated by data interpolation) and $t$-time of synoptic monitoring (days after source emplacement). In this case, $C_{o}$ is estimated as the maximum concentration observed on the curve $\left(C_{\max }\right)$. The estimate of groundwater velocity based on the 


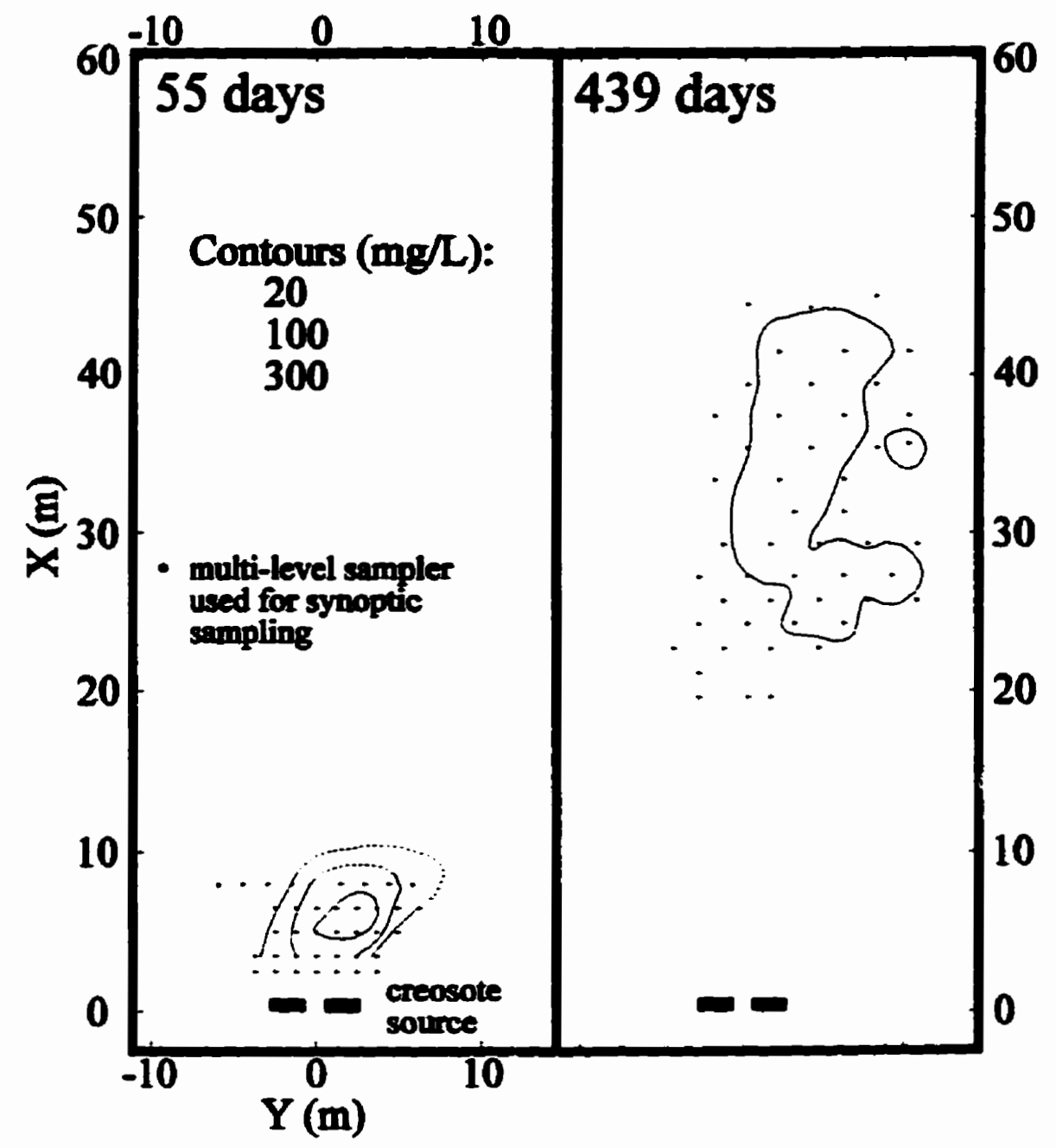

Figure 2.4. Contour plans of chloride concentrations at two different times after source emplacement; each plan indicates the multi-level samplers that were monitored; contours are based on the maximum concentration at each sampler. 


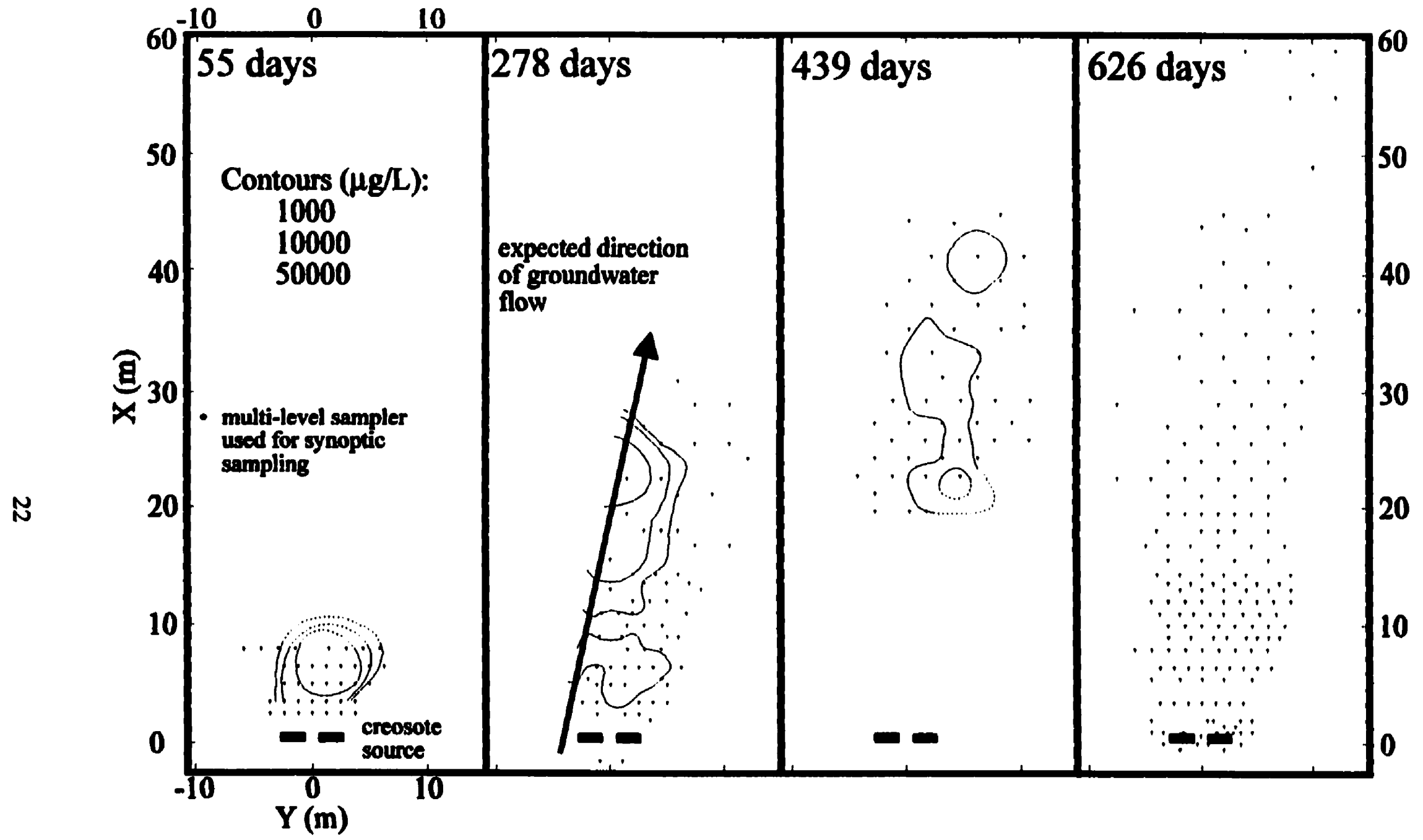

Figure 2.5. Contour plans of phenol concentrations at four different times after source emplacement; each plan indicates the multi-level samplers that were monitored; contours are based on the maximum concentration at each sampler. 


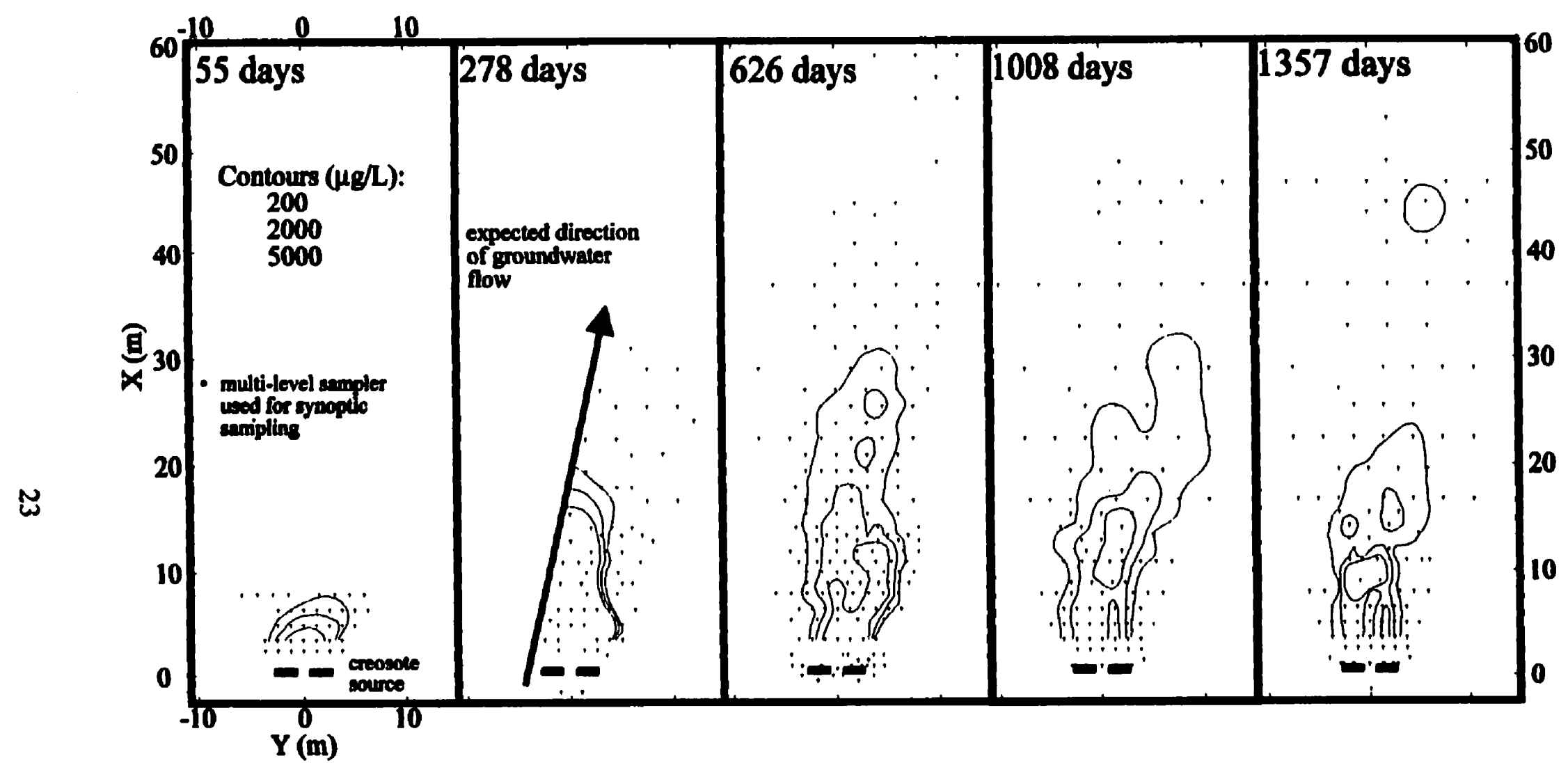

Figure 2.6. Contour plans of $m$-xylene concentrations at five different times after source emplacement; each plan indicates the multi-level samplers that were monitored; contours are based on the maximum concentration at each sampler. 


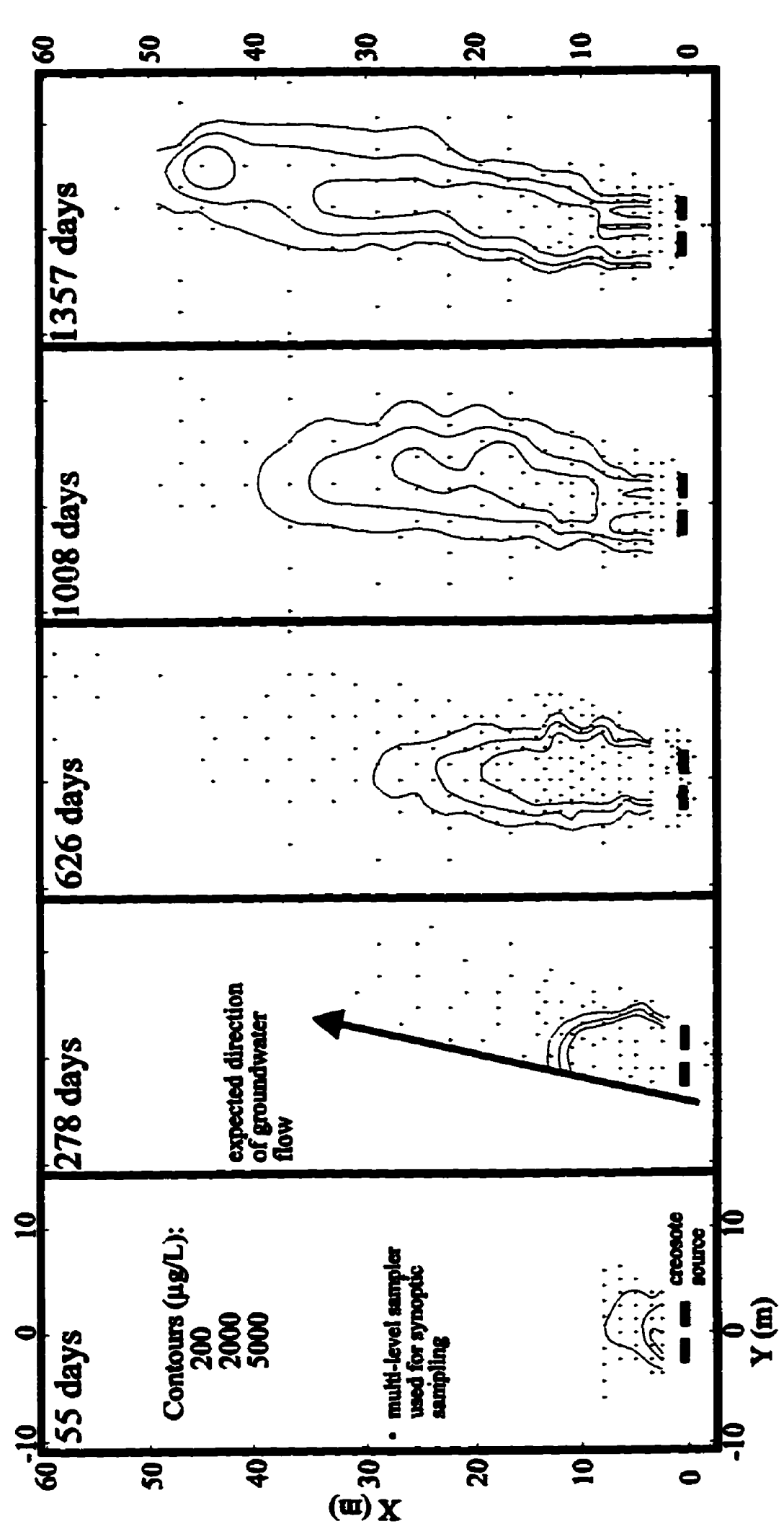

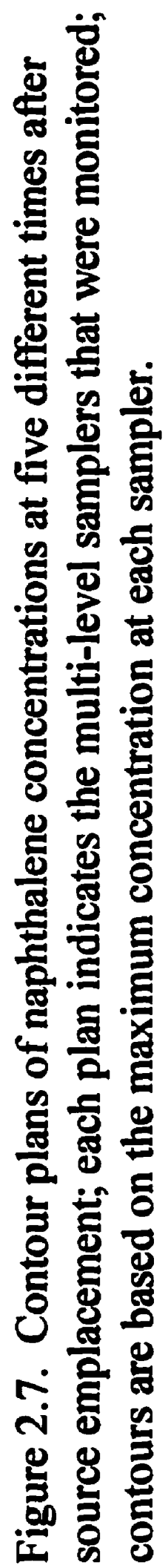




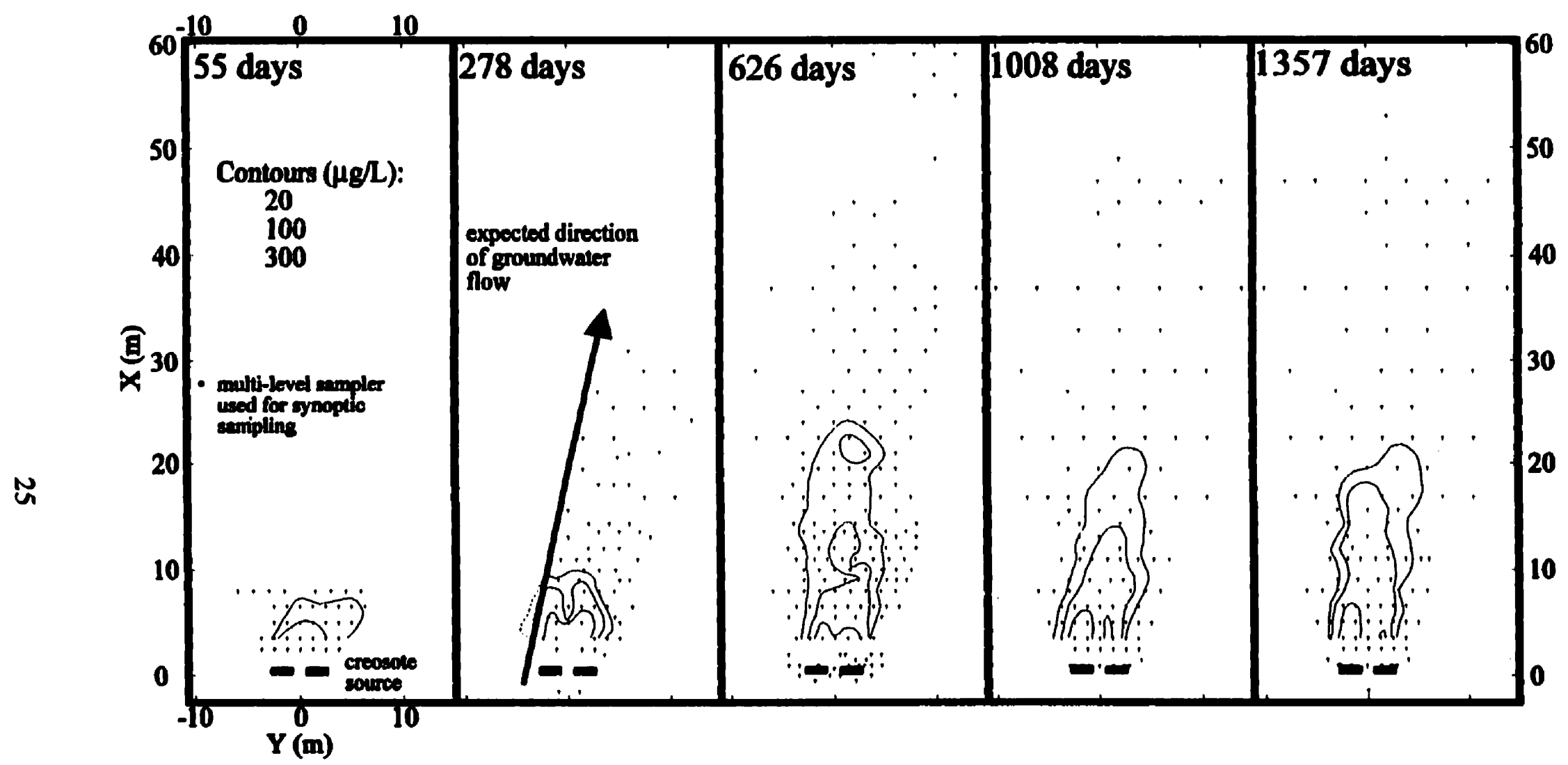

Figure 2.8. Contour plans of dibenzofuran concentrations at five different times after source emplacement; each plan indicates the multi-level samplers that were monitored; contours are based on the maximum concentration at each sampler. 


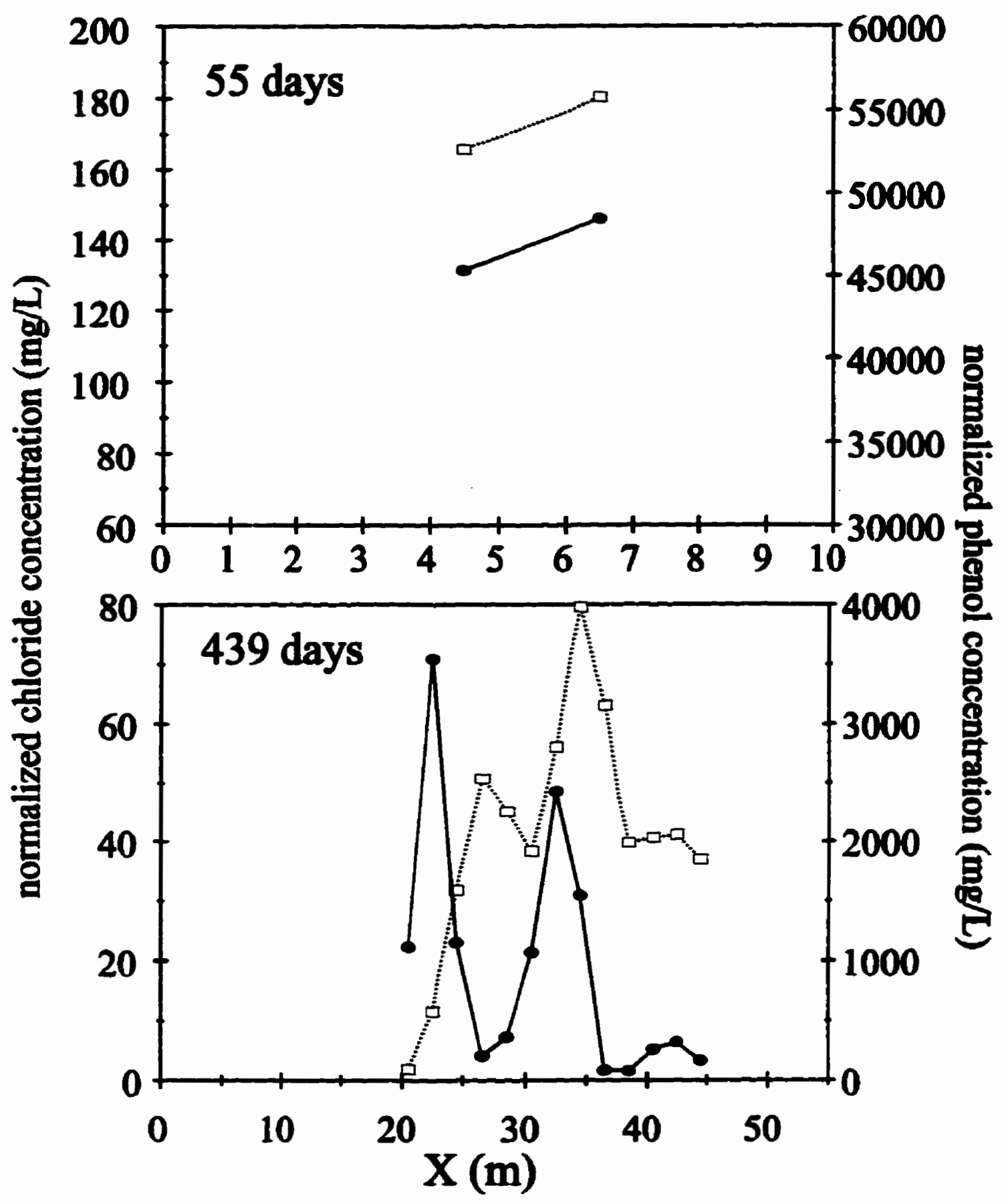

Figure 2.9. Profiles of dissolved chloride $(-(-))$ and phenol $(-\rightarrow)$ concentrations at 55 and 439 days after source emplacement; concentrations are normalized to the cross-sectional area of the creosote source (in the $\mathrm{Y}-\mathrm{Z}$ plane); profiles are in the approximate direction of groundwater flow. 


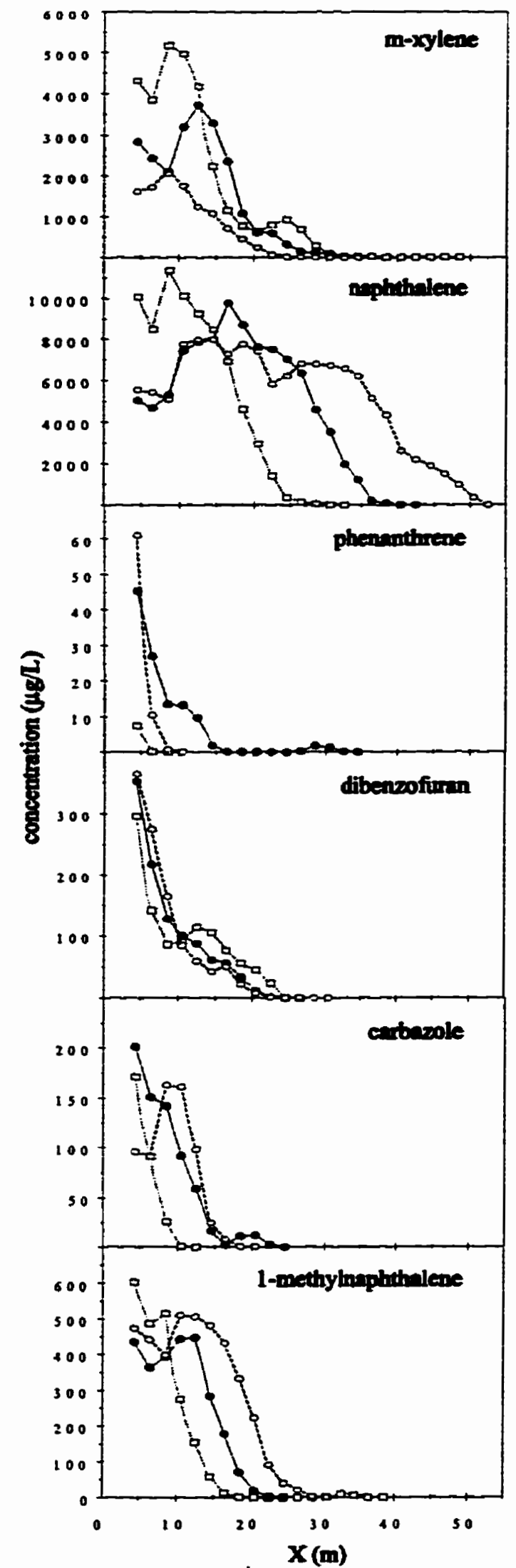

Figure 2.10. Profiles of dissolved concentrations of selected piume organics at 626 days $(\square), 1008$ days $\longleftarrow$ ) and 1357 days $(-\infty)$ after source emplacement, concentrations are normalized to the cross-sectional area of the creosote source (in the $\mathrm{Y}-\mathrm{Z}$ plane); and profiles are in the approximate direction of groundwater flow. 

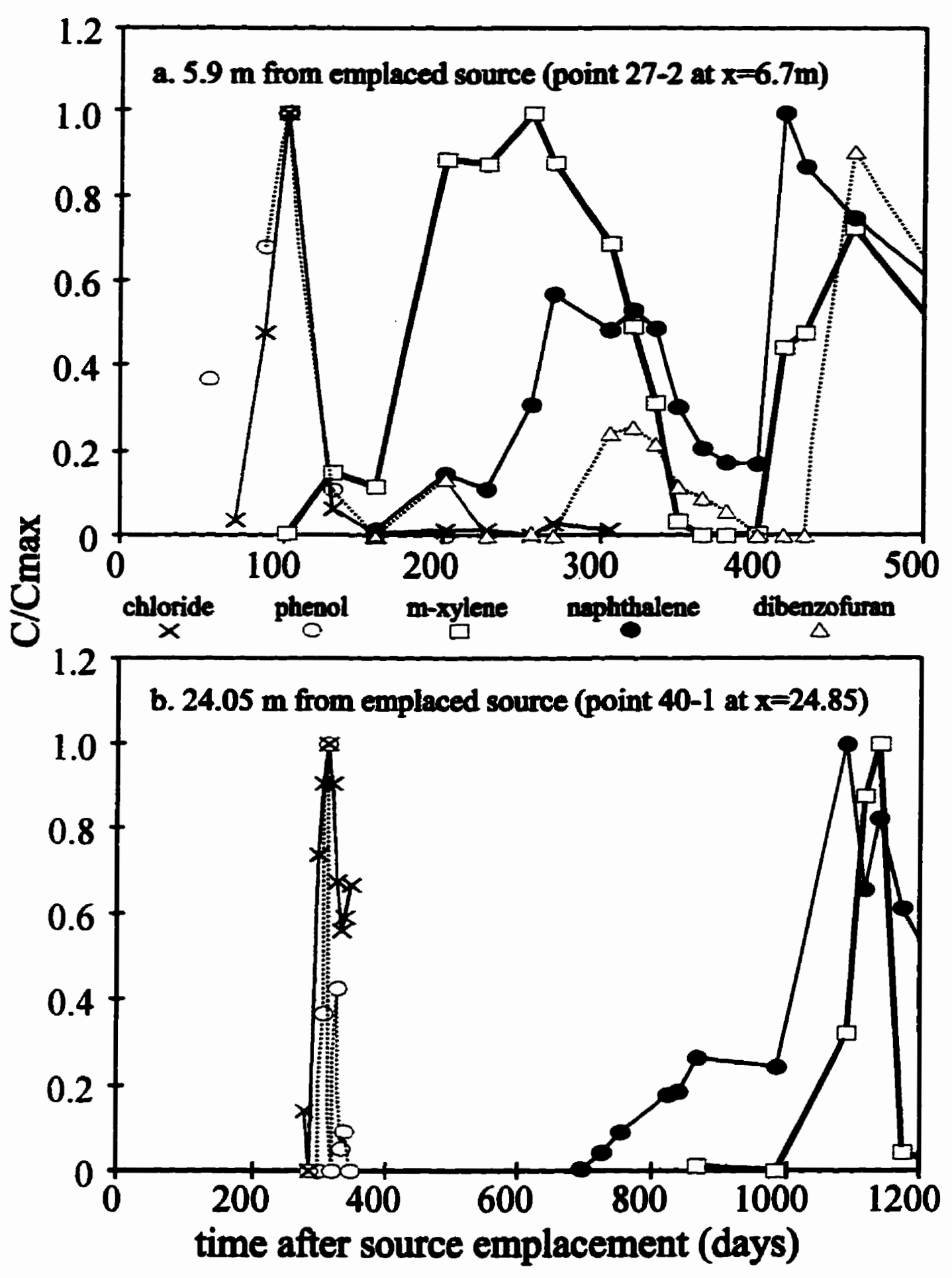

Figure 2.11. Time series plots for chloride and selected organics concentrations at two monitoring points downgradient of the emplaced creosote source. 
longitudinal chloride distribution profile at 439 days was $0.085 \mathrm{~m} /$ day and the parameter values used to calculate this velocity are shown in Table 2.6. Groundwater velocity was also estimated with chloride time series data from two selected points, shown in Figure 2.11. Again, equation (2.4) is used to calculate velocity; however, in this case $x$ was constant (at each point) while $t$ varied. Results, shown in Table 2.6, indicate a velocity of $0.066 \mathrm{~m} /$ day based on data from $x=6.7$ $\mathrm{m}$ and $0.083 \mathrm{~m} /$ day using data from $x=7.3 \mathrm{~m}$.

Table 2.6. Summary of data for estimation of average linear groundwater velocity

\begin{tabular}{lcc}
\hline Data type & $\begin{array}{c}\text { Approximate point at } \\
\text { which } C / C_{\text {max }}=0.5 \\
\text { occurs }\end{array}$ & $\begin{array}{c}\text { Corresponding velocity } \\
\text { estimate (m/day) }\end{array}$ \\
\hline $\begin{array}{l}\text { Synoptic monitoring at } \\
t=439 \text { days after source emplacement } \\
\text { (Figure 2.9) }\end{array}$ & $x=38 \mathrm{~m}^{1}$ & 0.085 \\
$\begin{array}{l}\text { Time series at } x=6.7 \mathrm{~m}^{1} \\
\text { (Figure 2.11) }\end{array}$ & $t=89$ days & 0.066 \\
$\begin{array}{l}\text { Time series at } x=24.85 \mathrm{~m}^{1} \\
\text { (Figure 2.11) }\end{array}$ & $t=290$ days & 0.083 \\
\hline
\end{tabular}

1 the downgradient boundary of the emplaced source occurs at $x=0.8 \mathrm{~m}$

Of the two types of measurements used to estimate $v$ above, the synoptic data (plume position at 439 days) are considered most appropriate for estimation of the plume-scale type of parameters that are of interest in this study: time series results are more susceptible to small-scale aquifer variability. However, all the velocity estimates are in reasonable agreement with estimates from Mackay et al. (1986), Hubbard et. al. (1994), and Patrick (1986), shown in Table 2.1. All three of these studies were based on injected plumes and were therefore able to utilize a preferred velocity estimation approach based on centre of plume mass, described by Freyberg (1986). Consequently, velocity determinations from previous studies at this site appear to be reasonable for application to the current study. Average linear groundwater velocity was subsequently used to estimate mass flux from the source to the plume (Chapter 3 ).

Phenol

As shown in Figure 2.3, phenol concentration in source leachate was expected to decrease relatively rapidly. This is due to the high solubility of this compound which causes it to be quickly depleted from the source. This is confirmed by Figure 2.5 which shows that by 55 days the 
phenol plume started to detach from the source. By 278 days, the plume migrated as a discrete slug and by 439 days, further spreading and a decrease in peak concentrations had occurred.

Extrapolation of the plume trajectory to 626 days would suggest that a significant portion of the plume should have been encountered in synoptic samples collected at this time; however, only isolated traces of phenol were detected. This suggests that the decrease in plume concentrations observed from 278 to 439 days continued, to the point that the compound was almost completely transformed at 626 days.

Comparison of the longitudinal distribution of phenol mass with that of chloride (Figure 2.9) supports the suggestion that significant transformation of phenol is occurring along the groundwater flow path. At 55 days, peak normalized concentrations of phenol and chloride are $48461 \mu \mathrm{g} / \mathrm{L}$ and $180 \mathrm{mg} / \mathrm{L}$, respectively. Later, at 439 days, it was apparent that the mass distribution of these two plumes is still similar in shape. However, peak chloride concentration had decreased to $79.6 \mathrm{mg} / \mathrm{L}$, approximately $44 \%$ of the 55 day concentration. Meanwhile, peak phenol concentration had decreased more sharply, to approximately $7 \%(3550 \mu \mathrm{g} / \mathrm{L})$. A quantitative mass balance is presented in Chapter 3.

Data from the two time series sampling points (Figure 2.11) indicate that the advancement of the phenol plume compared closely with chloride and therefore that the former was not significantly sorbed to Borden sand. However, synoptic data from 439 days (Figure 2.9) provides a more detailed basis for evaluating a retardation factor $(R)$ for phenol. The similar, but slightly offset, curves of phenol and chloride mass distribution at 439 days indicate a slight degree of phenol retardation, which can be estimated according to:

$R=d_{c} / d_{p h}$

where $d_{c l}$ the distance from the source to a given point on the chloride curve and $d_{p h}$ is the distance from the source to the corresponding point on the phenol curve. This method provides an $R$ estimate for phenol of approximately 1.05 . However, given the evidence of phenol mass loss, this can be considered an upper limit for $R$ : mass loss from the front of the plume would cause an apparent decrease in the rate of plume migration.

\section{M-xylene}

Comparison of time series curves for $m$-xylene and chloride (Figure 2.11) show that the migration of the former was apparently retarded with respect to the conservative tracer (chloride). At monitoring point $27-2$ ( $x=6.7 \mathrm{~m} ; 5.9 \mathrm{~m}$ from source) an $R$ of approximately 2.5 was indicated by the lag between the curves which, in this case, is estimated by:

$R=t_{C} t_{\text {org }}$

where $t_{C l}=$ time required to reach maximum chloride concentration and $t_{\text {org }}=$ time required to 
reach maximum organic compound concentration. Meanwhile, a similar calculation for 40-1 $(\mathrm{x}=24.85 \mathrm{~m})$ yielded an $R$ of 3.7 . This increase in $R$ was probably due to transformation of $m$ xylene along the flow path, to the degree that the plume migration is curtailed. Inspection of the $m$-xylene plume contour plans (Figure 2.6) shows that plume reached a maximum observed extent at 627 days and then the plume front was observed to withdraw back toward the source at 1008 days and further again at 1358 days.

The observation of $m$-xylene plume drawback, is also shown by the plot of longitudinal mass distribution in Figure 2.10. This figure also shows a decrease in plume mass over time, providing evidence of transformation. Evidence of decreasing $m$-xylene flux into the plume is shown by lower concentrations at the influent end of the plume (which has been delineated at $x=2.7 \mathrm{~m}$ ). This trend is in general agreement with the expected trend of $m$-xylene dissolution, shown in Figure 2.3. These observations suggest that the plume has drawn back due to a combination of $m$-xylene transformation within the plume and decrease in $m$-xylene influx from the source. That is, the rate of flux decreased to below the rate of plume transformation, leading to a decrease in the size and mass of the plume.

\section{Naphthalene}

The plan views of naphthalene plume development (Figure 2.7) show a relatively consistent rate of plume growth and, as of 1358 days, the plume was still expanding. Comparison of longitudinal mass distribution profiles (Figure 2.10) shows that plume mass also increased during this period. The near-source concentrations on the profiles are evidence that the strength of source leachate, with respect to naphthalene, had decreased between each of the last three snapshots, which is in general agreement with the trend predicted by Raoult's law (Figure 2.7). However, it is evident that there was apparently still sufficient flux of naphthalene into the plume to support plume expansion.

Time series sampling results (Figure 2.11) show that naphthalene was retarded with respect to chloride migration. At the point closest to the source $(x=6.7 \mathrm{~m})$, the arrival of the naphthalene peak lagged behind that of the chloride peak, indicating an $R$ of approximately 2.6 . Further downgradient $(x=24.05 \mathrm{~m})$ this factor increased to 3.5 , indicating that the forward progression of the naphthalene plume may have decreased somewhat with time, although this is not apparent in the plume contour plans.

\section{Dibenzofuran}

Time series monitoring at $x=6.7 \mathrm{~m}$ shows that the migration rate of dibenzofuran is slower than that of chloride by an $R$ of 3.12. The compound was not detected at the other selected time series monitoring point. Plan views of the dibenzofuran plume (Figure 2.8) show that it did not migrate appreciably in the two year duration between the last three synoptic monitoring events and this 
observation is also supported by the longitudinal concentration profiles in Figure 2.10. The profiles also show that dibenzofuran flux into the plume was similar at the time of the last two synoptic sampling events, which is consistent with the nearly constant rate of source dissolution predicted by Raoult's law and shown in Figure 2.8. The lower flux indicated for 627 days in Figure 2.10 may be due to the higher $R$ and the associated lag time required for the plume to migrate into the monitored zone. There is strong evidence that the dibenzofuran plume is at steady state, where mass flux into the plume is balanced by mass transformation within the plume.

\section{Phenanthrene}

This compound was more highly retarded than naphthalene and $\boldsymbol{m}$-xylene and was not detected at the two selected time series sampling points. Near source concentrations of phenanthrene in the longitudinal concentration profiles (Figure 2.10) indicate that flux of this compound into the plume increased over the last three snapshots. This probably has less to do with an increase in source strength than with the highly retarded plume front gradually moving into the monitored zone. After relatively rapid expansion of the phenanthrene plume from 627 to 1008 days, it was observed to recede and decrease in mass, as of $\mathbf{1 3 5 8}$ days.

\section{Carbazole}

Longitudinal profiles of carbazole concentration in Figure 2.10 show that the plume advanced over the period from 627 to 1008 days but remained relatively stable from 1008 to 1358 days. Unlike dibenzofuran, carbazole loadings into the plume have not been consistent over the monitoring period. Near-source concentration reached an observed maximum of approximately $200 \mathrm{ug} / \mathrm{L}$ at 1008 days and then decreased to approximately $100 \mathrm{ug} / \mathrm{L}$ at 1358 days, which is generally consistent with the decline in source leachate concentration predicted by Raoult's law (Figure 2.3). The low near-source concentration at 627 days may be due to a lag time required for the plume to migrate into the monitored zone.

\section{1-Methylnaphthalene}

Development of the plume for this compound followed a trend similar to that of naphthalene. Inspection of plume longitudinal profiles in Figures 2.10 indicate that plume position advanced and plume mass increased at a relatively consistent rate over the monitoring period. Also similar to naphthalene, the rate of flux into the plume decreased over time although to a lesser degree than naphthalene. This dissolution trend is generally consistent with the Raoult's law prediction. However, in comparison to naphthalene, the rate of plume migration was slower. 


\section{CHAPTER 3 \\ MASS BALANCE}

\section{BACKGROUND}

The occurrence of compound transformation is often a critical issue in the evaluation of organic plumes. Even relatively slow rates of transformation may impose significant limitations on the extent of plume migration, compared to the situation where decreases in organic solute concentration are due only to sorption and hydrodynamic processes. The environmental risk associated with groundwater contamination is closely related to the distance to which contamination may migrate away from the source, and evaluation of this distance requires an estimate of the rate and nature of solute transformation. Methods for evaluating plume transformation are generally based on some form of mass balance, using one-, two-, or threedimensional data, depending on the simplicity of plume and source geometry and the nature of the study.

Probably the most widely applied technique of evaluating plume transformation is a one dimensional approach whereby solute concentrations are monitored along an inferred groundwater flowline. In order to attribute a portion of an observed concentration decrease to compound degradation, the effects of hydrodynamic dilution and sorption must be taken into account. Barker et al. (1986) studied a landfill leachate plume in North Bay, Ontario, and compared concentrations of selected organics along an assumed groundwater flow line to evaluate relative rates of organics degradation. Using this technique, they concluded that under anaerobic conditions $o$-xylene was transformed more rapidly than ethyl benzene and that significant degradation of trimethylbenzene occurred only in the last third of the plume, where strictly anaerobic conditions may not persist. The authors pointed out that comparison between organics and a conservative tracer in the plume (chloride) could have provided a means of calculating an absolute rate constants. However, evidence that the chloride input flux did not vary with that of the organics meant that there was no basis for comparison at down gradient plume monitoring points.

Lyngkilde and Christensen (1992a) were able to use chloride concentrations to correct organics concentrations for hydrodynamic dilution in their study of a landfill leachate plume in Vejen, Denmark. They determined that major degradation of selected organics (BTEX compounds, naphthalenes, C-3 benzenes, benzene isomers, bicyclo compounds and chlorophenols) occurred within $50 \mathrm{~m}$ of the landfill. Most of the organics were not detected within $100 \mathrm{~m}$. Using a conceptually similar approach, Baedecker et al. (1993) evaluated the behaviour of dissolved volatile organic carbon (DVOC) composed primarily of BTEX compounds, as part of an intensive characterization of an oil spill in Bemidji, Minnesota. DVOC was compared with $\mathrm{Ca}^{2+}$, which was conservative along part of the flowpath, and to a modelled conservative tracer. They concluded that a marked decrease in DVOC along a $41 \mathrm{~m}$ flowpath was mostly due to plume biodegradation, and not dispersion. 
Ehrlich et al. (1982) used sodium as a conservative tracer in a study of a coal tar distillation and wood-treating plant in St. Louis Park, Minnesota. By comparison, they determined that the concentration of phenolic compounds decreased dramatically, by a factor of approximately 150 in a groundwater migration distance of $430 \mathrm{~m}$ while sodium decreased by a factor of only four. Moderate degradation was indicated for naphthalene, with concentration decreasing by a factor of 10. Goerlitz et al. (1985) reported on an extensive characterization of groundwater contamination at a wood-preserving facility in Pensacola, Florida. They compared organics concentrations in samples collected at increasing distances from the source and determined that phenol and single-substituted phenols (2-, 3- and 4-methyl phenol) decreased disproportionately to PAHs. They attributed this behaviour to more rapid environmental biodegradation of the former and they also concluded that higher substituted phenols (2,4- and 3,5-dimethyl phenol) were essentially conservative at the site since they behaved similar to chloride. (Godsy et al., 1992).

The two-dimensional approach to plume mass balance involves determination of total plume mass passing through one or more transverse sections and then comparison of mass between the source and a section, or between two sections. Thierrin et al. (1995) used a unique two dimensional approach for determining plume mass loss. A solution of deuterated benzene, toluene, $p$-xylene and naphthalene was injected into a groundwater plume that originated from an underground gasoline storage tank. Injection water also contained bromide as a conservative tracer. The researchers monitored the passage of injected solutes past a row of multi-level piezometers located $17 \mathrm{~m}$ from the injection point. By comparing bromide and organics mass, they were able to evaluate for organics transformation and to estimate degradation rates.

In a similar approach Barbaro et al. (1992) injected two slugs of gasoline-contacted water into a landfill leachate plume under anaerobic, denitrifying conditions. One slug was amended with nitrate to evaluate the effect of denitrification on biodegradation of aromatics. Plume mass was calculated by integrating solute breakthrough curves at sequential rows of multilevel samplers. Results for BTEX compounds showed complete disappearance of toluene from the unamended plume within five metres of the injection point while the other compounds were recalcitrant under these conditions. Transformation of ethyl benzene and xylenes was detected in the nitrateamended plume and benzene was recalcitrant in both plumes, within the monitored distance.

The three dimensional mass balance approach requires monitoring data from the entire plume, to compare total plume mass at two or more times. Three previous studies have utilized dense three dimensional monitoring to formulate mass balances for injected plumes within the current study area. Mackay et al. (1986) injected water containing five halogenated organic chemicals and two inorganic tracers (chloride and bromide) into the Borden aquifer. The mass of the two inorganic tracers remained relatively constant (Freyberg, 1986). Total plume mass was also conserved with respect to carbon tetrachloride and tetrachloroethylene, while three of the organics (bromoform, 1,2-dichlorobenzene and hexachlorethane) showed evidence of significant transformation (Curtis et al., 1986). Barker et al. (1987) followed the degradation of an injected plume of benzene, 
toluene and xylene isomers (BTX). They observed essentially complete degradation of all BTX compounds within 434 days after injection, and observed similar disappearance rates for all aromatics except benzene, which was the only compound to persist after 270 days. Hubbard et al. (1994) conducted simultaneous injection of three organic solute plumes composed of water that had contacted: 1) gasoline only 2) gasoline and methyl-tertiary butyl ether (MTBE) and 3) gasoline and methanol. Over a sixteen month monitoring period, MTBE exhibited no mass loss while only one percent of the methanol remained at the end of the monitoring period. The BTEX compounds were degraded in all plumes with the most rapid rate noted for toluene and the slowest for benzene.

MacIntyre et al. (1993) conducted a three dimensional mass balance of an injected plume containing benzene, $p$-xylene, naphthalene and $o$-dichlorobenzene, at Columbus Air Force Base in Mississipi. Significant mass loss was determined for all four organics with the most rapid rate noted for $p$-xylene and the slowest rate for o-dichlorobenzene.

The purpose of this component of the current study is to evaluate a complex organic plume for plume-scale evidence of mass transformation. A main objective of the study design was to simulate a scenario typical of many contaminated industrial sites: a fixed source of organic contamination that dissolves slowly into groundwater over time. Implementation of this emplaced source experiment under controlled field conditions made it possible to formulate compound mass balances from relatively dense three dimensional data, not typically possible at an actual contaminated site.

\section{METHODS}

\section{General approach}

Perhaps the most straightforward approach for quantifying compound transformation is to compare initial mass in the source with mass remaining at various times in the sorbed, dissolved and residual (within the source) phases. These values could then be used to estimate the amount of transformed mass for a given compound at any time $(t)$ after source emplacement according to:

$M_{T}=M_{0}-\left(M_{R}+M_{p}\right)$

where $M_{T}=$ transformed mass, $M_{0}=$ initial mass of compound in the creosote source (at $\left.\mathrm{t}_{0}\right), M_{R}=$ mass of compound remaining in source (in residual creosote) at time $=t ; M_{P}=$ total mass associated with the plume $=M_{D}+M_{S}: M_{D}=$ dissolved mass in the plume and $M_{S}=$ sorbed mass associated with the dissolved plume.

However, there are difficulties in applying this approach. $M_{0}$ is well known, since the original quantity of creosote added to the source was measured and the creosote composition was determined by analysis. But, since $M_{R}$ can be so large compared to other components in the mass 
balance $\left(M_{n}, M_{D}\right.$ and $\left.M_{S}\right)$, the magnitude of error in the estimate of the former may be in the same range as expected values for the latter components. This is illustrated by Table 3.1 which shows the confidence interval of data from source cores collected at 626 days after emplacement, compared to dissolved plume mass $\left(M_{D}\right)$. It is apparent that the uncertainty in estimating $M_{R}$ (by coring the source) is potentially much greater than $M_{D}$, for most compounds. The largest discrepancy tends to occur for the most hydrophobic compounds, since their lower solubility limits the rate at which the dissolved phase increases.

Table 3.1. Summary of analytical data for sand cores collected from the source in conjunction with synoptic sampling at 626 days ( $\mathrm{n}=23$ from six cores).

\begin{tabular}{lccc}
\hline \multicolumn{1}{c}{ Compound } & $\begin{array}{c}\text { Mass in Source } \\
(\mathrm{g})\end{array}$ & $\begin{array}{c}95 \% \text { Confidence Interval }^{2} \\
(\mathrm{~g})\end{array}$ & $\begin{array}{c}\text { Dissolved Plume }^{(\mathrm{g})} \\
\text { phens }\end{array}$ \\
\hline m-xylene & 0 & 0 & trace \\
naphthalene & 530 & 78 & 135 \\
phenanthrene & 5453 & 397 & 333 \\
dibenzofuran & 9709 & 472 & 0.033 \\
carbazole & 2879 & 174 & 4.65 \\
1-methylnaphthalene & 934 & 61 & 1.30 \\
\hline
\end{tabular}

1 based on average concentration $(\mathrm{mg} / \mathrm{kg}) \times$ mass of source sand $(-6018 \mathrm{~kg})$; data in Appendix 2

$2 \quad$ expressed as "equivalent" source mass based on Confidence Interval (mg/kg) $x$ mass of source sand

3 from dissolved phase monitoring; presented here for comparison purposes; more complete results in Table 3.3.

Consequently, an alternative mass balance approach has been used, where $M_{T}$ was estimated for the periods ( $\Delta t^{\prime}$ 's) between synoptic sampling events only on the basis of dissolved phase concentrations, sorption characteristics and groundwater flux, according to:

$$
M_{T}=M_{F}-\Delta M_{P}
$$

and

$$
F=A \int_{t_{2}}^{t_{1}} F d
$$

where $F=$ mass flux into the plume for the given compound $\left(\mathrm{M} / \mathrm{T} / \mathrm{L}^{2}\right) ; A=$ the cross-sectional area of the source transverse to the direction of groundwater flow $\left(\mathrm{L}^{2}\right)$ and $M_{F}=$ the quantity of mass input over the integration period (M). Figure 3.1 is a diagram illustrating the various components 


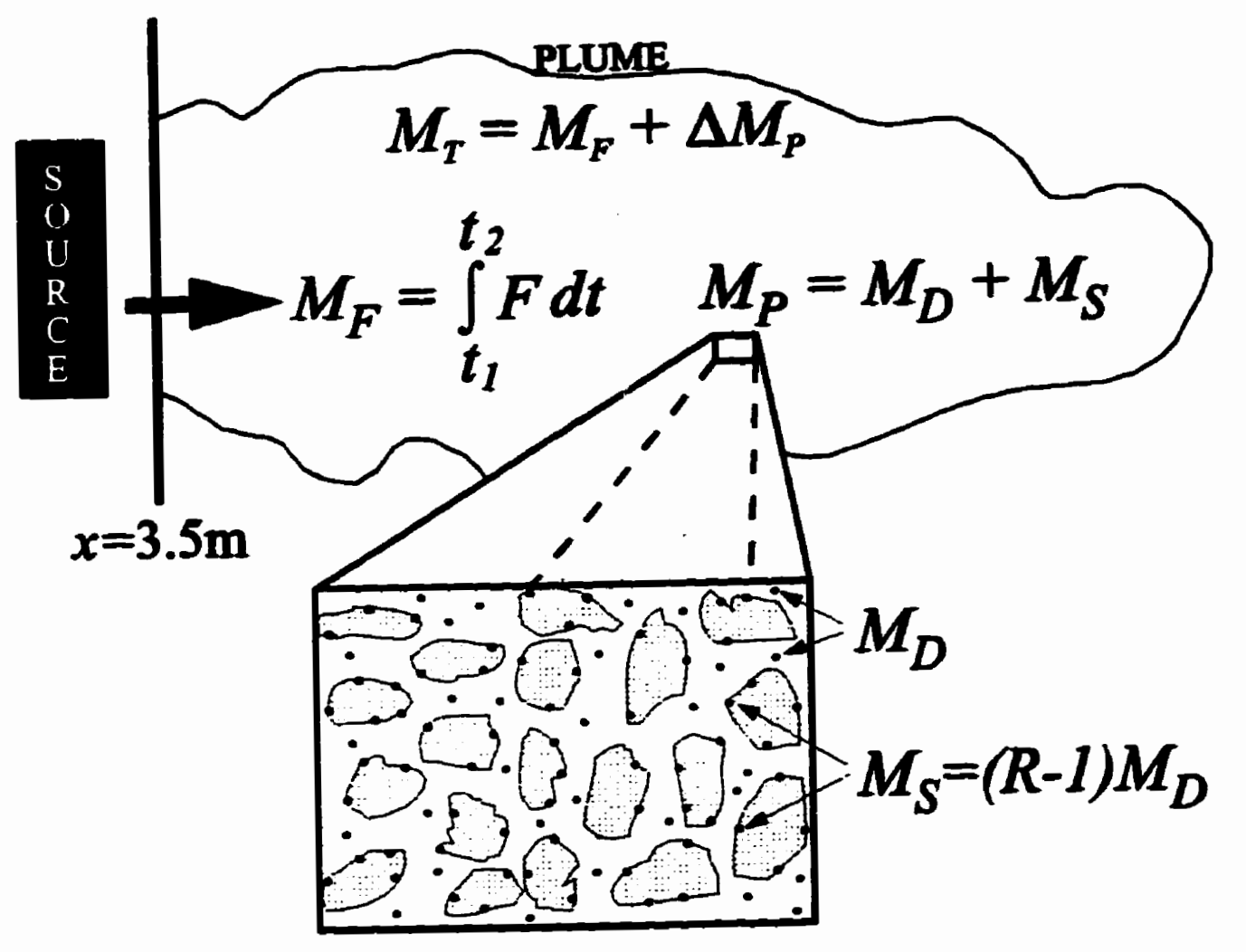

Figure 3.1. Conceptualized cross-section showing the main components of the mass balance for the plume from the emplaced creosote source. 
of this mass balance approach. As shown, the balance relates to that portion of the plume located at $x>3.5 \mathrm{~m}$. The plume has been defined in this way to avoid the large errors associated with interpolation of concentration data nearer the source, where lateral concentration gradients are relatively large. The following describes methods for quantifying each component of the mass balance.

\section{Dissolved mass $\left(M_{D}\right)$}

Synoptic plume sampling was conducted at six times since source emplacement $(t=55,278,439$, 640,1008 and 1357 days) and sufficient plume coverage for calculation of total dissolved mass was obtained for the latter four of these. A description of the analytical methodology is given in Chapter 2 with additional details provided in Appendix 4 and detailed data provided in Appendix 2.

Dissolved mass has been calculated using a three dimensional interpolation routine in the ENTEC (Surpac Inc., 1995) geostatistical program whereby irregularly spaced three dimensional data are interpolated to a regularly spaced array of three dimensional cells. In the approach used, the influence of each data point on the interpolated cell value is proportional to the inverse of the cubed distance between the data point and the point at the centre of each cell. The search radius for inclusion of data points into calculation of a given cell point is specified on the basis of the dimensions of an ellipsoid centred at the cell point. In this case, the major axis of the ellipsoid was oriented in the $x$-direction and had a length of $16 \mathrm{~m}$. The minor axis of the ellipsoid was eight metres, oriented in the $y-z$ plane. Sensitivity analysis of the search radius indicated that this parameter was relatively insensitive, due to the cell point weighting function based on the inverse of distance cubed. Grid $x, y$ and $z$ spacings of two, one and $0.5 \mathrm{~m}$, respectively, were selected as optimal on the basis of a sensitivity analysis that showed that smaller spacings did not cause significant changes to calculated dissolved mass.

In many cases, low but non-zero, organics concentrations were detected at top and/or bottom sampling points, indicating incomplete plume closure. Where this occurred, the location of the plume periphery was approximated by adding "dummy" points of zero concentration at the top and/or bottom of the given multi-level sampler at the typical vertical interval of the sampling network $(20 \mathrm{~cm})$. Review of vertical concentration profiles in these cases generally indicated that the error introduced into plume mass calculations by this procedure should be negligible because: 1) concentration trends generally decreased towards the top and bottom indicating that these extreme points were approaching the plume periphery and 2) the top and/or bottom of the plume was observed at other multi-level piezometer locations.

Similarly, where the plume was detected at either end of a row of multilevel samplers, points of zero concentration were added at a horizontal distance equal to the spacing of the samplers in the given row. Review of plume distributions along the given row, and nearby rows, indicated that the locations of these zero points were reasonable and, therefore that the associated error should 
be negligible. In general the mass estimate error introduced by incomplete plume closure should be minor since the placement locations of the added points was reasonable on the basis of proximal data, and also because large majority of plume mass is distributed in the highly concentrated central core of the plume, rather than the periphery, where concentrations tend to be low.

After grid interpolation, dissolved plume mass for a given compound was calculated according to:

$M_{D}=n \Delta x \Delta y \Delta z \Gamma C$

where $n=$ aquifer porosity, $C=$ concentration at the cell centre point $\left(g / \mathrm{m}^{3}\right) ; \Delta x, \Delta y$ and $\Delta z$ are grid spacings in the $x, y$ and $z$ dimensions, respectively (m). A value for $n$ of 0.33 was used on the basis of parameter evaluation by Mackay et al. (1986).

Previous studies carried out at the current study site have used conservative tracers to evaluate the accuracy of plume mass estimates based on samples from the groundwater monitoring network. These studies indicated that plume mass was in reasonable agreement with initial mass and, therefore, that samples from the monitoring network can provide a reasonable estimate of plume mass. Freyberg (1986) reported on results of co-injection of bromide and chloride with organic compounds, followed by plume monitoring over 1038 days (bromide) and 647 days (chloride). Plume mass determinations for bromide $(n=13)$ showed that the average and variance of relative mass estimates were 0.89 and 0.20 , respectively. Similar measures for the chloride plume $(n=12)$ were 0.90 and 0.16 , respectively. Patrick (1986) co-injected chloride with BTEX compounds and subsequently determined chloride plume mass at five times over 108 days. The average mass of the plume relative to injected mass was $\mathbf{0 . 7 8}$. In a similar experiment, Hubbard et al. (1994) co-injected chloride with three different mixtures of methanol and gasoline compounds. Chloride plume masses, determined at either four or five times over a period of 476 days, showed a range in average relative mass from 0.88 to 1.11 for the three plumes. Although a conservative tracer (granular $\mathrm{NaCl}$ ) was initially placed in the source used for the current study, it was not suitable for evaluating the accuracy of plume mass estimates: initial mass was not known. However, conservative tracer results from the three previous studies discussed above provide evidence that the monitoring approach can provide a reasonable estimate of dissolved mass.

\section{Sorbed Mass $\left(M_{S}\right)$}

Due to the hydrophobic nature of the compounds in creosote, a degree of sorption will occur from the aqueous phase to aquifer solids. Furthermore, depending on the hydrophobicity of the compound and the sorbent properties of the aquifer, the quantity of sorbed mass in a given volume of aquifer can be substantially greater than the mass in solution. Consequently, sorbed mass has potential to be an important component of the mass balance. Sorption is often approximated as a linear reversible equilibrium process, also known as sorption ideality, whereby dissolved concentration $(C)$ is related to sorbed concentration $(\Omega)$ by a solids partitioning 
coefficient $\left(K_{d}\right)$, as follows:

$S=K_{d} C$

where $K_{d}$ is in units of $\mathrm{L}^{3} / \mathrm{M}$ and can be used to calculate a retardation factor $(R)$ by:

$R=l+(\rho / \eta) K_{d}$

where $\rho=$ bulk density of the porous medium $\left(M / L^{3}\right)$. Sorbed mass can then be estimated with $R$, according to:

$M_{s}=(R-1) M_{D}$

Although the assumption of sorption ideality is often applied, laboratory and field experiments have often provided evidence of solute behaviour that deviates from ideality. A thorough review of processes that can lead to these types of deviations is provided by Brusseau and Rao (1989). These authors discuss the significant effects of physical phenomena, such as rate limitations due to diffusion of solute into low hydraulic conductivity particle aggregates or laminae. They also point out the difficulty in estimating the parameters that may control these processes.

In the current study, it was assumed that sorption was reasonably approximated by the assumption of ideality, on the basis of previous studies at the site. Curtis et al. (1986) compared laboratory sorption data and field results from an injected plume of bromoform, carbon tetrachloride, tetrachloroethylene, 1,2-dichlorobenzene and hexachlorethane injected at the Borden site. They estimated $K_{d}$ by sorption batch testing and by monitoring the migration rate of the piume centre-of-mass. It was concluded that the close agreement of these methods was evidence that the behaviour of the study compounds could be approximated by sorption ideality, although the authors noted that the methods agreed more closely when the plume was near the source. A similar comparison was conducted by Hubbard et al. (1994) for BTEX compounds in a plume of dissolved gasoline injected at the same site. This group of compounds also showed behaviour that was generally consistent with sorption ideality.

Actual values of $K_{d}$ for the study compounds were estimated by three different methods: 1) empirical correlation 2) laboratory batch test and 3) comparison of groundwater and aquifer solids from within the plume. The first method is widely used and is described by Karickhoff (1984). The basis of this approach is that the the organic fraction $\left(f_{o c}\right)$ is the primary sorbate for hydrophobic compounds in subsurface systems. Further, the partitioning relationship is likened to partitioning of dissolved hydrophobic compounds from an aqueous phase to a liquid organic phase. Accordingly, sets of empirical relationships have been developed to equate partitioning of an organic compound between octanol and water (described by $\boldsymbol{K}_{\text {oww }}$ ), to a partitioning between water and a given earth material (described by $K_{d}$ ), according to:

$\log K_{d}=a \log K_{o w}+\log f_{o c}+b$ 
where $a$ and $b$ are empirically derived coefficients. In this case, coefficients for $a$ and $b$ of 0.72 and 0.49 were used and were obtained from empirical correlation experiments conducted by Schwarzenbach and Westall (1981) which included two representative compounds (naphthalene and pyrene) from the seven studied in this experiment. Table 3.2 shows a compilation of $K_{d} s$ calculated by this method for the compounds of interest in the Borden study. An $f_{o c}$ value of 0.0002 was used, on the basis of measurements by Mackay et al. (1986), as shown in Table 2.1. Values for logK $K_{\text {ow }}$ are shown in Table 2.4.

Sorption coefficients determined by laboratory batch testing are also provided in the table. To conduct these tests, Borden sand was placed in $100 \mathrm{~mL}$ glass bottles, in contact with serial dilutions of creosote-saturated water. Sorption was inferred from the difference in aqueous concentration between bottles containing sand and bottles with no sand (controls). A relatively low sand to liquid ratio was used $(\sim 0.2 \mathrm{~g} / \mathrm{mL})$ so that the aqueous concentrations of the more hydrophobic compounds would remain well above analytical detection limits. However, since this sand:solution ratio did not produce significant changes in aqueous concentrations for the least hydrophobic of the study compounds ( $m$-xylene and phenol), $K_{d} s$ were not determined for these compounds. Detailed methodology and results from laboratory batch tests are provided in Appendix 8.

Table 3.2. Estimates of solids partitioning coefficients $\left(K_{d}^{\prime} \mathbf{s}\right)$ for selected compounds and Borden sand.

\begin{tabular}{|c|c|c|c|c|c|}
\hline \multirow[t]{2}{*}{ Compound } & \multirow[t]{2}{*}{ Batch test } & \multirow{2}{*}{$\begin{array}{l}\text { In situ } \\
\text { measurement }\end{array}$} & \multirow{2}{*}{$\begin{array}{c}\text { Based on } \\
\log K_{\text {ow }}^{1}\end{array}$} & \multicolumn{2}{|c|}{ Borden tracer tests } \\
\hline & & & & $\begin{array}{r}\text { Patrick } \\
\text { (1986) }\end{array}$ & $\begin{array}{c}\text { Hubbard et al. } \\
\text { (1994) }\end{array}$ \\
\hline phenol & - & - & $0.01(1.05)$ & $\overline{-}$ & - \\
\hline m-xylene & - & - & $0.11(1.6)$ & 0.13 & $0.06-0.09$ \\
\hline naphthalene & $0.22(2.2)^{2}$ & 0.09 & 0.16 & - & - \\
\hline phenanthrene & $1.80(10.87)$ & 4.7 & 1.11 & - & - \\
\hline dibenzofuran & $0.67(4.67)$ & 1.26 & 0.57 & - & - \\
\hline carbazole & $0.83(5.55)$ & 0.94 & 0.29 & - & - \\
\hline 1-m.naphthalene & $0.24(2.31)$ & 0.41 & 0.37 & - & - \\
\hline $\log K_{d}=0.7$ & $K_{o w}+\log f_{\alpha}+$ & (Schw & $h$ and & & \\
\hline
\end{tabular}

For the third method of $K_{d}$ estimation, aquifer cores were collected at three plume locations. Each location was within $30 \mathrm{~cm}$ (horizontally) of multi-level samplers that showed consistent aqueous concentrations over at least three adjacent sampling levels $(40 \mathrm{~cm})$. Entire $30 \mathrm{~cm}$ core sections $(5$ $\mathrm{cm}$ diameter) from the middle of this range were analysed for selected creosote compounds. $K_{d}$ 's 
were then calculated for each compound at each location by re-arranging equation (3.5) to:

$K_{d=} S / C$

where, in this case, $C=$ mean aqueous concentration from the three sampling points that span the vertical range of the adjacent core $\left(\mathrm{M} / \mathrm{L}^{3}\right)$ and $S$ is sorbed concentration $(\mathrm{M} / \mathrm{M})$ calculated according to:

$S=\left(\boldsymbol{F}_{T}-w M \boldsymbol{F}_{T} C\right) \boldsymbol{F}_{S}$

where $M_{T}=$ total mass of compound in sample $(M) ; M_{T}=$ total mass of sample $(M) ; M_{S}=$ mass of solids in sample and $w=$ sample water content (fraction).

$M C_{T}$ was quantified by extracting the entire cored interval three times with dichloromethane, concentrating the combined extracts in a roto-evaporator and then injecting into a Gas

Chromatograph, using a method similar to that for extracts from aqueous samples, as discussed in Chapter 2. Complete results of core analysis are provided in Appendix $9 . K_{d}$ s calculated by this technique are shown in Table 3.2. Values were not calculated for $m$-xylene and phenol: dissolved concentrations of the former compound showed a relatively large degree of vertical variation at the time of coring ( 1245 days after source emplacement) and the latter had essentially disappeared from the monitoring network.

$K_{d}$ 's determined by the empirical method have been used for estimating sorbed compound masses for $m$-xylene and phenol, in subsequent mass balance calculations. In the case of $\boldsymbol{m}$-xylene, this value was in the range of $K_{d}$ 's derived at the field site through earlier tracer tests by Patrick (1986) and Hubbard et al. (1994), shown in Table 3.2. For phenol, both the empirical correlation approach and comparison of migration rates for the phenol plume relative to the chloride plume (Chapter 2) indicate that this compound undergoes only minor sorption in Borden sand. It will be shown that in the context of the mass balance, the quantity of sorbed phenol mass was minor compared to dissolved mass, initial source mass and transformed mass.

Results from the laboratory batch method were used for the five remaining compounds: naphthalene, phenanthrene, dibenzofuran, 1-methylnaphthalene and carbazole. Earlier field studies at the site have shown that batch results compared reasonably well with observed plume behaviour. Although none of the five compounds were used in these field studies, a wide range of compounds are represented (nonchlorinated aromatics, chlorinated aromatics and aliphatics) and behaviour is expected to be similar. Batch testing provided $K_{d}$ s in the range of the empirical correlation method for the first four compounds listed above, providing an indication that the sorption coefficients are reasonable. For carbazole, the two methods provided significantly different results. The carbazole $K_{d}$ value derived from batch testing is used in mass balance calculations, on the basis that it is expected to be more site-specific. 
The method of evaluating sorption by comparing sorbed and aqueous phase concentrations from within the plume is conceptually the most attractive of the three methods since it is site-specific and should also allow for the relatively long time that some of the compounds have been in contact with the aquifer. However, the analytical uncertainty inherent in the analysis of sorbed concentrations is difficult to quantify. Consequently, while the approach showed promise, additional method development is required to apply the results. This method provided the largest estimate of sorbed mass for all compounds except naphthalene.

\section{Mass Flux $(F)$}

Mass entering the plume has been estimated relative to an up gradient plume boundary defined at $x=3.5 \mathrm{~m}$ ( $2.7 \mathrm{~m}$ from the source), which is the location of a row of multilevel samplers. The boundary has been defined at this location because steep concentration gradients at closer proximity to the source lead to large errors in data interpolation. Mass flux across the plume boundary at a given time $(t)$ is given by:

$F=q C_{y z}$

where $q=$ groundwater flux $(\mathrm{L} / \mathrm{T})=v n$, and $C_{y z}=$ plume concentration at $x=3.5 \mathrm{~m}$, integrated

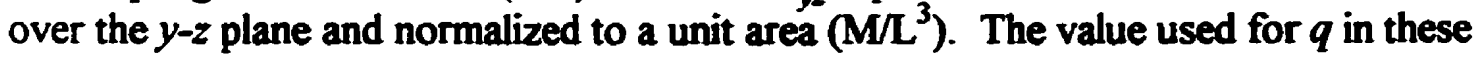
calculations was $0.03 \mathrm{~m} /$ day on the basis of past site work that evaluated average linear groundwater velocity $(v)$ and porosity $(n)$. As discussed in Chapter 2, data from past studies and the current study indicate that $v$ in the vicinity of the study site is relatively consistent over time. In addition, expected changes to the local flow regime caused by the presence of the more permeable source sand were evaluated with the model FLOWPATH (Waterloo Hydrogeologic Software, 1994) according to source and ambient hydraulic parameters described in Chapter 2. Model results, shown in Figure 3.2, indicated that minimal perturbation of flowlines was expected in the immediate vicinity of the source and that flowlines (and therefore $v$ ) should return to the up gradient configuration within two metres down gradient of the source. This conclusion is supported by time series and synoptic data for a conservative tracer (chloride) placed in the source. They show that groundwater moved into the monitoring network from the vicinity of the source at a velocity that is in reasonable agreement with past studies conducted at the site (Chapter 2).

$C_{y z}$ was determined for each synoptic sampling event, with plume concentrations collected from a row of multilevel samplers located at $x=3.5 \mathrm{~m}$, by approximating the following:

$C_{y z}=(I /(\Delta y \Delta z)) \int-_{\infty}^{\infty} \int-\infty{ }^{-\infty} C(y, z) d y d$,

where $\Delta y \Delta z=$ the area over which the integrated concentration is expressed. The approximation was calculated using trapezoidal quadrature, first in the $z$ direction and then in the $y$ direction, with the limits of both integrations set outside the plume periphery. This method is similar to that 


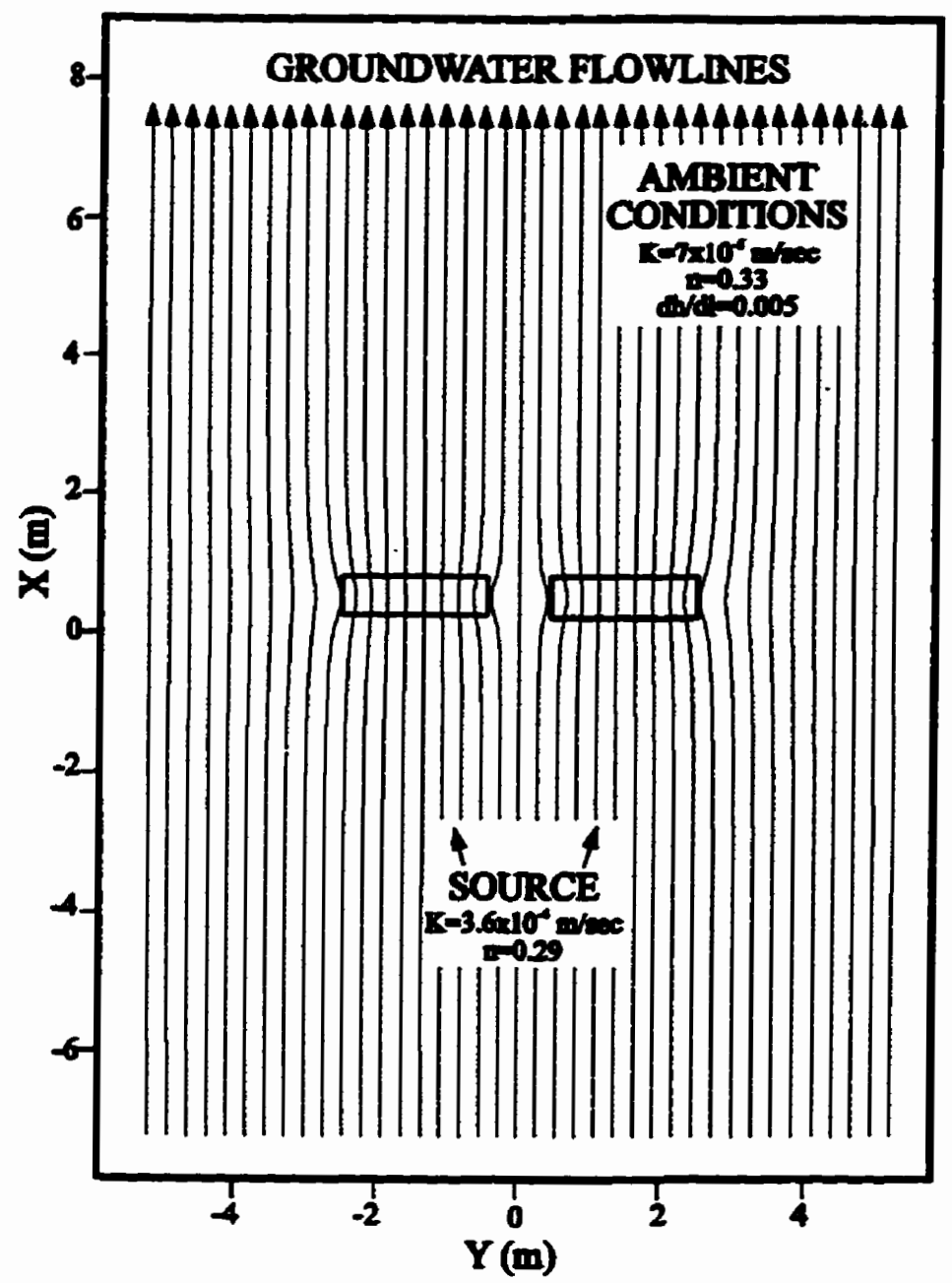

Figure 3.2. Plan view of groundwater flowlines in the vicinity of the emplaced creosote source, as generated by the FLOWPATH model. Results indicate that the source has negligible effect on flowlines beyond $2 \mathrm{~m}$ downgradient of the source. 
used by Freyberg (1986) in an earlier study at the same site, except in the earlier work it was applied only in the $z$ direction. Application in the $z$ direction is conducted by application of the following for each multilevel sampler in the row

$$
C_{z}=\frac{1}{2 I} \sum_{z=1}^{n-1}\left(z^{i+1}-z\right)\left(C^{i+1}+C^{i}\right)
$$

where $C_{z}=$ vertically integrated concentration for each multilevel sampler $\left(\mathrm{M} / \mathrm{L}^{3}\right), I_{z}=$ vertical thickness over which the integrated concentration is expressed (unit $L$, in this case), $z^{I}=z$ coordinate of bottom sampling point $(\mathrm{L})$ and $C^{t}=$ concentration at bottom sampling point. The subsequent approximation in the $y$ direction is calculated with:

$$
C_{y z}=\frac{1}{2 I_{y}} \sum_{i=1}^{n-1}\left(y^{i+1}-y^{i}\right)\left(C_{z}^{i+1}+C_{z}^{\prime}\right)
$$

where $C_{y z}=$ horizontally ( $y$ direction) and vertically integrated concentration for the row of multilevel samplers $\left(\mathrm{M} / \mathrm{L}^{3}\right), I_{y}=$ horizontal width over which the integrated concentration is expressed (unit $\mathrm{L}$, in this case), $y^{l}=y$ coordinate of the westernmost multilevel sampler (L) and $C_{z}^{l}=$ vertically integrated concentration for the westernmost multilevel sampler. For both equations, the limits of the approximation are unimportant, as long as they extend beyond the plume periphery.

Total compound mass entering the plume between synoptic sampling events $\left(M_{F}\right)$ is given by:

$$
M_{F}=\int_{t_{1}}^{t_{2}} F d t=q \int_{t_{1}}^{t_{2}} C_{t=} d t
$$

where $q$ is outside the integral since it assumed to be constant. This expression was approximated by:

$M_{F}=\left(q\left(C_{y=}{ }^{1}+C_{y z}{ }^{2}\right) \Delta t\right) / 2$

which further assumes that interpolation of $C_{y z}$ between synoptic sampling events can be reasonably approximated by a straight line. Profiles of dissolved concentration versus distance from the source, shown in Figure 2.10, indicate that mass influx to the plume has changed gradually over time, and that straight line interpolation of mass flux was a reasonable assumption. Application of a dissolution model based on Raoult's law, discussed in Chapter 2 and shown in Figure 2.3, also suggested that a straight line approximation was reasonable. 


\section{MASS BALANCE RESULTS}

Mass balance results for the seven selected compounds are summarized in Table 3.3 and transformation rates, expressed in terms of half lives, are provided in Table 3.4. Half-lives have been estimated with an iterative model where total plume mass $\left(M_{\text {TOT }}\right)$ at the end of a given time step was calculated on the basis of dissolved mass $\left(M_{D}{ }^{l}\right)$, according to:

$M_{\text {TOT }}{ }^{\prime}=M_{D}{ }^{\prime}(R-1)+M_{D} e^{-\lambda \Delta t}$

where the superscript " $I$ " indicates parameter values at the end of the time step, $\lambda$ is the first order decay constant (time $e^{-1}$ ) which is related to half life $\left(t_{1 / 2}\right)$ by $t_{1 / 2}=\ln (2) / \lambda$ and $\Delta t$ is the length of the time step. First order decay has been assumed for the purpose of describing plume mass loss because many field and laboratory studies of biodegradation have found that it is reasonably described as a first order process. (e.g., Barker et al., 1987; MacIntyre et al., 1993; Nielsen et al., 1996; Wilson et al., 1990; Thierrin et al., 1995). The first term on the right hand side of equation (3.17) provides an estimate of sorbed mass and the second term represents dissolved mass at the end of the time step, after compound transformation has occurred. Calculation of $M_{\text {TOT }}{ }^{l}$ by this equation assumes that only the dissolved phase undergoes transformation. $M_{D}{ }^{\prime}$ in this model was evaluated by:

$$
M_{D}^{1}=\frac{q \Delta t\left(C_{y z}^{0}+\Delta C_{y z}\right)}{R}+M_{D}^{0}
$$

where the first term on the right hand side accounts for the new mass added to the plume over the time step, incorporating sorption of a portion of that mass through division by $R$. For the first time step $C_{y z}{ }^{0}$ is calculated by integration of plume monitoring data from $x=3.5 \mathrm{~m}$ and $\Delta C_{y z}$ is the change in $C_{y z}$ over the time step assuming linear interpolation between synoptic monitoring events. $M_{D}{ }^{0}$ is initially obtained from synoptic monitoring data for the whole plume and, for subsequent time steps is calculated according to:

$M_{D}^{0}=M_{T}^{l} / R$

\section{DISCUSSION}

Mass balance calculations indicate that all the selected compounds undergo transformation in the plume. However, estimates of transformed mass and transformation half-lives are generally considered more reliable where little change occurs in mass flux between synoptic sampling events. Large changes in mass flux have more potential to introduce error since the flux may deviate significantly from the assumption of linear interpolation. 
Table 3.3. Summary of mass balance input data and results for the emplaced creosote source plume.

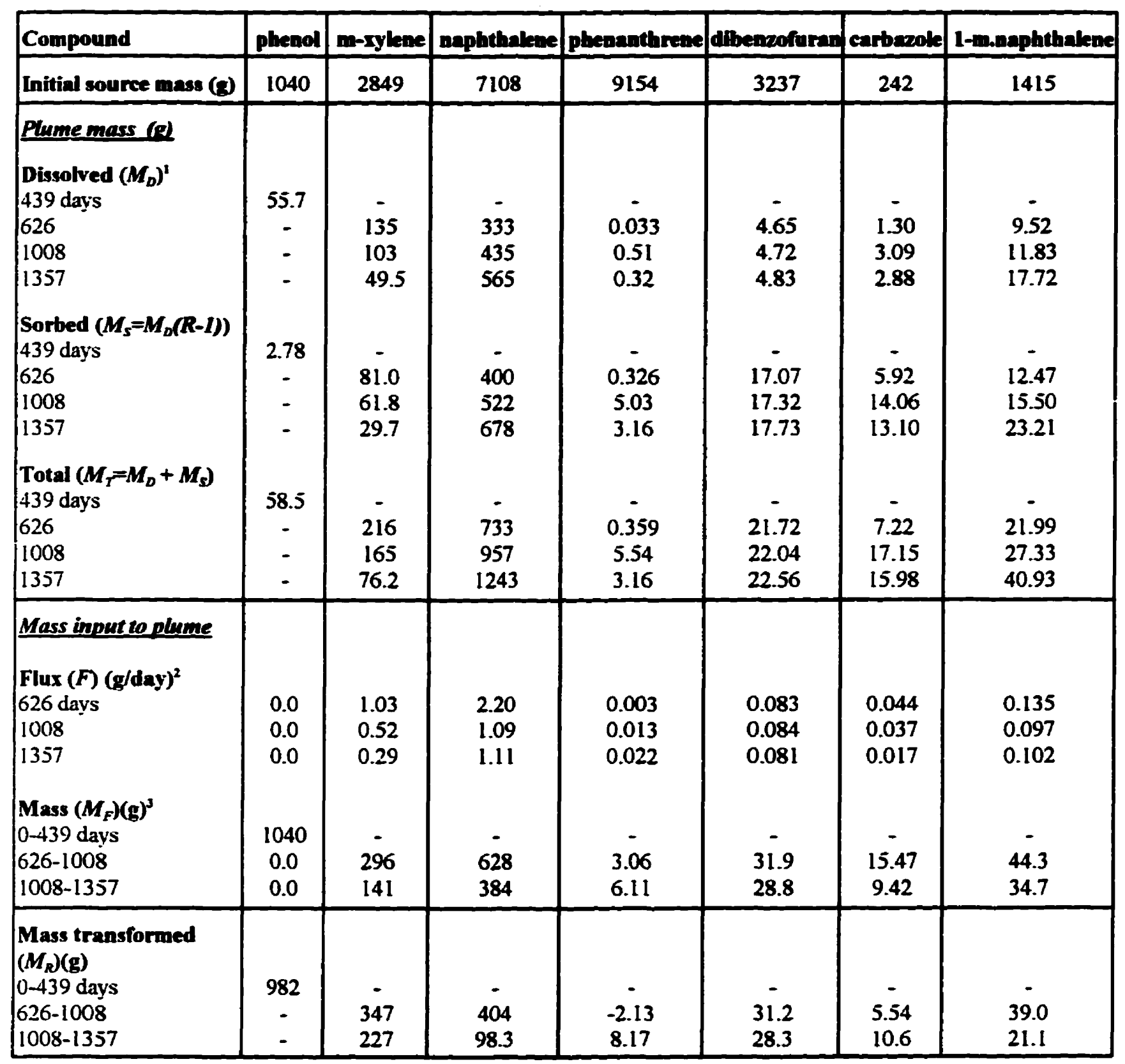

Calculated from synoptic monitoring data

$F=q C_{y z}$, where $q=v / n, v=0.091 \mathrm{~m} / \mathrm{day}, n=0.33$ and $C_{y z}$ is calculated with synoptic monitoring data from the base of the plume (defined at $x=3.5 \mathrm{~m}$ ).

Assumes linear interpolation of $F$ between synoptic monitoring events 
Table 3.4. Half-lives (in days) for selected plume compounds

\begin{tabular}{lccc}
\hline Monitoring period: & $0-439$ days & $626-1008$ days & $1008-1357$ days \\
\hline phenol & 99 & - & - \\
m-xylene & - & 95 & 78 \\
naphthalene & - & 265 & 1215 \\
phenanthrene & - & not available & 11 \\
dibenzofuran & - & 40 & 41 \\
carbazole & - & 110 & 71 \\
l-methylnaphthalene & - & 78 & 173 \\
\hline
\end{tabular}

For $m$-xylene, naphthalene, phenanthrene and l-methylnaphthalene, estimates of transformed mass and transformation rates were considered most reliable for the period between the last two monitoring events (from 1008-1357 days). Estimates of carbazole transformation were considered most reliable for the period between 626 and 1008 days since mass flux estimates were comparable at the beginning and end of this period. The decrease in carbazole flux at 1357 days is probably due to depletion of carbazole from the source. Mass flux of dibenzofuran was comparable for the last three synoptic sampling events and, consequently, estimates of mass transformation are considered reliable for the two intervening periods.

Mass balance quantification confirmed the qualitative observations of plume behaviour in Chapter 2. Dibenzofuran formed the most stable plume, with nearly constant rates of mass flux between the last three synoptic sampling events (Table 3.3) and relatively constant dissolved mass. The dibenzofuran plume approached steady state over the study period, where mass flux was approximately balanced by mass transformation. The rate of dibenzofuran transformation is relatively constant over time and the interaction of mass flux and transformation are serving to keep the plume from advancing further. Presumably, the dibenzofuran plume expanded until the mass flux rate was matched by the plume-scale rate of transformation. To the author's knowledge this is the first conclusive observation of an organic plume approaching a steady state condition that does not involve groundwater discharge. The data from this site illustrate that natural transformation processes can halt the advancement of an organic plume, even in the presence of an ongoing source.

Carbazole was also not expected to advance further since the transformed mass is greater than the mass input, for the last monitoring period (1008-1357 days). However, in this case plume growth is limited by a decrease in mass flux into the plume, rather than an increase in the overall rate of mass transformation. As shown in Table 3.3, the original source concentration of carbazole was the lowest of the selected compounds, and the aqueous solubility of carbazole is relatively large: $238.6 \mathrm{mg} / \mathrm{L}$, as shown in Table 2.4. Consequently, the decrease in carbazole flux between the last two synoptic sampling events was attributed to significant source depletion with respect to this compound. 
A similar process has controlled the development of the $m$-xylene plume. This plume reached a maximum observed plume mass at 626 days and was subsequently observed to shrink, as the rate of mass flux decreased to below the rate of mass transformation. The rapid decrease in the rate of mass flux is attributable to the relatively large solubility of $\mathbf{m}$-xylene which caused it to become depleted from the source. With more constant flux, the $m$-xylene plume would likely have approached steady state over the monitoring period, similar to dibenzofuran.

For naphthalene, the rate of mass accumulation exceeds the rate of transformation and, given the relatively small magnitude of the latter, the plume is expected to grow considerably larger before the two rates approach a balance. Similarly, the 1-methylnaphthalene mass flux also exceeds transformation and it is expected that significant additional plume growth will occur.

Mass balance results indicate that almost all of the original mass of phenol in the source (approximately one kilogram) has been transformed: by day 439, the phenol plume contained only six percent of the mass originally present in the source. Interpretation of the phenol mass balance is relatively straightforward because unlike the other selected compounds, phenol was rapidly and completely leached from the source early in the study period. Furthermore, it was subjected to only minor sorption to aquifer solids. The rate calculated for this transformation ( $t_{1 / 2}=99$ days) is considered a minimum, since it was assumed that all phenol was instantaneously leached from the source into the plume at the beginning of the experiment.

Unlike the other compounds, phenanthrene flux has continued to increase up to the last synoptic sampling event. This is attributed to the higher retardation factor for this compound, which has increased the time required for it to break through to the monitored portion of the plume. The large relative difference in flux rates between days 626 and 1008 have caused large error in mass flux calculations for the intervening period, giving rise to a negative estimate for the quantity of mass transformed. The estimate of phenanthrene mass transformation for the subsequent period (1008-1357 days) is more reasonable but may still contain significant error due to the assumption of linear interpolation between flux measurements. Perhaps the most straightforward evidence of phenanthrene transformation is demonstrated by the decrease in total plume mass between 1008 and 1357 days. Total mass decreased from 5.54 to $3.16 \mathrm{~g}$ and, even without considering flux input over this period, this represents a half life of $t_{1 / 2}=49$ days. 


\section{CHAPTER 4 \\ BIODEGRADATION INDICATORS}

\section{BACKGROUND}

A central issue at many sites where groundwater is affected by organic contaminants is whether contaminants are undergoing some degree of transformation as they migrate in groundwater. Even a relatively small rate of transformation may impose considerable limits on the extent of plume growth, which can lessen the potential for human or environmental exposure to plume contaminants. Many types of organic contaminants can be utilized in the metabolic pathways of naturally existing subsurface microorganisms, as discussed in a number of review articles. Atlas (1988) reviewed the potential of petroleum hydrocarbon for microbial biodegradation and concluded that microbes capable of of mediating this process were widespread in the environment. Mueller et al. (1989) provided a similar review for polycyclic aromatic hydrocarbons (PAHs), phenolic compounds and N-, S- and $\mathbf{O}$ - heterocyclics: the three main compound types found in creosote. They cited a wide range of studies that documented the biodegradability of these compounds. In general, biodegradation of non-chlorinated organics like those discussed above, is favoured under more oxidizing conditions. However, a host of studies, as outlined in several review articles (e.g., Grbic-Galic, 1989; Grbic-Galic, 1990a; Bollag and Kaiser, 1991), have shown that PAHs, monoaromatic, phenolic and heterocyclic compounds have been observed to biodegrade under various anaerobic conditions (denitrifying, sulphate-reducing, methanogenic and fermentative).

While the ability of naturally occurring microbes to mediate transformation of nonchlorinated organics is well supported by laboratory studies there are limitations on direct transferral of these results to the plume-scale. Evaluation of organic plume biodegradation is made difficult by the complexity of transport and degradation processes in a dynamic groundwater system and, as noted by Madsen (1991), are further complicated by problems inherent in formulating a mass balance in spatially heterogeneous media typically found in the field. Madsen reviewed several studies of field biodegradation and noted that these were usually based on multiple lines of independent evidence. These studies started with field evidence of contaminant mass loss and then compared relevant parameters inside and outside the contaminated zone to evaluate for the type of changes that would be expected if the mass loss was microbially mediated.

The portion of the study discussed in this chapter is conceptually similar to the "typical" approach in that it starts with field-scale evidence of plume transformation: the mass balance formulated in Chapter 3. Consequently, the study design provides novel and reliable evidence for the important first step of determining in situ biodegradation - evidence of mass loss - against which other types of evidence can be evaluated. Since appreciable transformation of several plume compounds was indicated, additional lines of investigation have been followed to evaluate for biodegradation. Laboratory microcosms were used to determine whether the study compounds are biodegradable under simplistic laboratory conditions. Groundwater at the field site was monitored for electron 
acceptors and organic acids to evaluate changes expected with biodegradation. In addition, aquifer solids were examined for indications of microbial adaptation in response to the presence of the plume.

\section{LABORATORY MICROCOSMS}

\section{Methods}

A microcosm experiment was conducted to determine if indigenous aquifer microorganisms from within the creosote plume were capable of metabolizing compounds in an aqueous solution representative of the creosote plume. The experiment was conducted under aerobic conditions since this was expected to be most favourable for biodegradation of the selected compounds and therefore the most likely to produce observable mass loss. In the field, it is expected that the core of the plume is depleted in oxygen, and that expectation is borne out by field measurements presented later in this paper. Consequently, the microcosm redox condition was probably most representative of field conditions on the periphery of the plume, where it mixes with background groundwater.

The microcosms were $1100 \mathrm{~mL}$ glass bottles sealed with O-ring tap stopcocks (J. Young Scientific Glassware Ltd.). The bottles were also equipped with side arms capped with mininert valves to allow for repeated sampling while maintaining an atmospheric seal. All equipment used during the microcosm setup was sterilized and aseptic techniques were employed throughout the experiment. The sand used in the microcosms was obtained from a relatively concentrated portion of the plume, approximately $3.3 \mathrm{~m}$ downgradient of the creosote source, at the location shown on Figure 4.1, 1246 days after source emplacement. Plume data indicated that sand at this location was exposed to six of the seven selected compounds. The exception was phenol: this compound had completely leached from the source and passed by this point approximately two years earlier. Sand core was obtained in $5 \mathrm{~cm}$ diameter aluminum core barrels (sterilized prior to use) by the wireline coring method described by Zapico et al. (1987). In the laboratory, sand was extruded from the core barrel in a sterile air flow cabinet and accumulated in sterile 1L largemouth jars. After mixing, $140 \mathrm{~g}$ of core material was added to each of three of the microcosm bottles that had been designated as sterile controls. These bottles, and their contents, were sterilized by autoclaving for one hour on three separate occasions over a five day period. Unsterilized core material was added directly to six bottles to compose six active microcosms.

The aqueous solution used in the microcosms was prepared by contacting uncontaminated Borden groundwater with a creosote mixture similar to that used in the field: $6 \mathrm{~g} \mathrm{~m}$-xylene and $0.95 \mathrm{~g}$ carbazole added to $150 \mathrm{~mL}$ of raw creosote. The creosote mixture was placed in a $6 \mathrm{~L}$ erlenmeyer flask with $4 \mathrm{~L}$ of Borden groundwater and stirred for 48 hours on a magnetic stirrer. At the end of this period, creosote contacted groundwater was carefully siphoned off and diluted with clean Borden groundwater by approximately two times. Each of the nine microcosms received $380 \mathrm{~mL}$ of this solution and the three control microcosms also received $3.4 \mathrm{~mL}$ each of a 


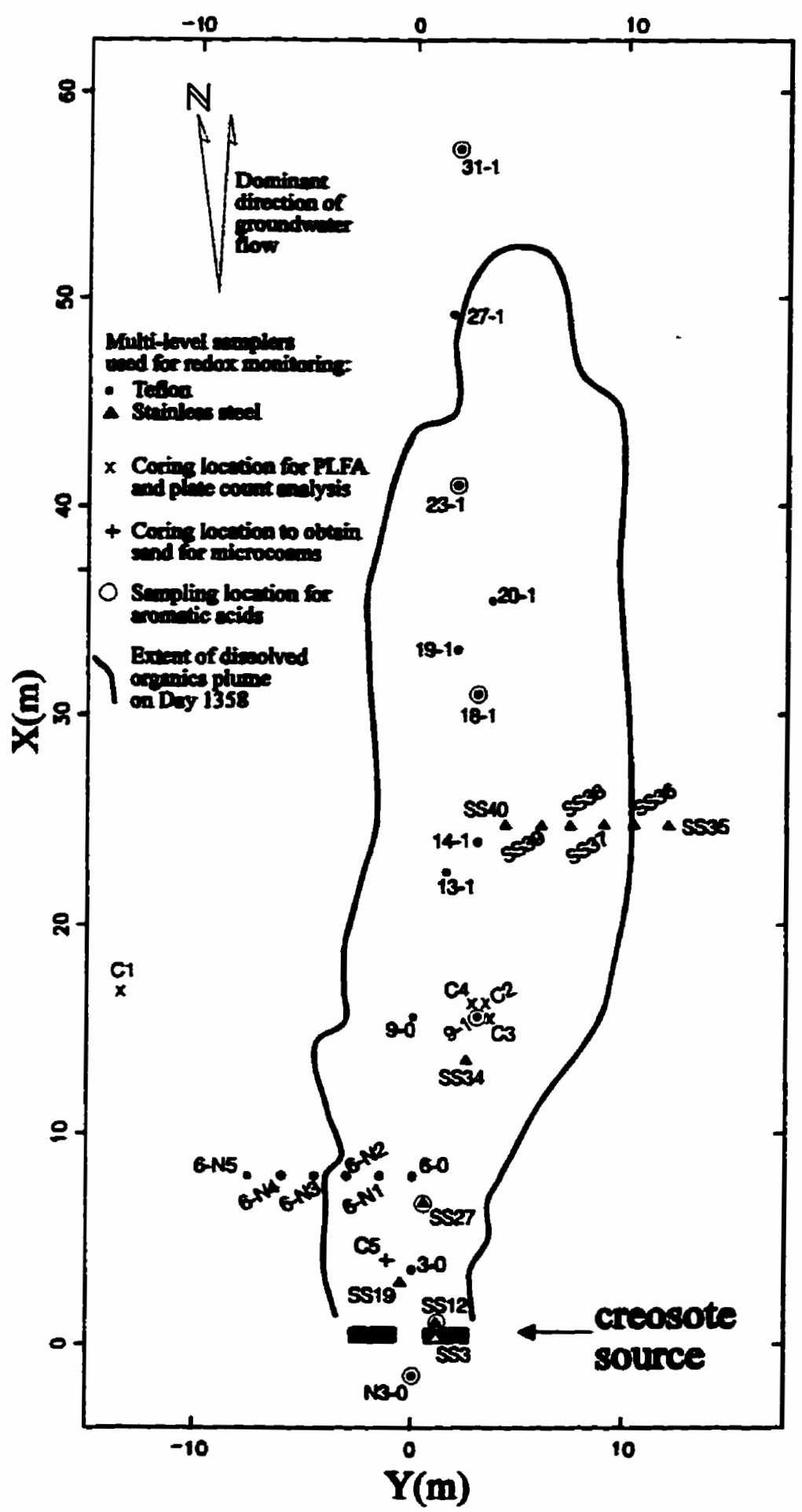

Figure 4.1. Plan showing plume monitoring locations for selected redox parameters, aromatic acids and microbiological indicator parameters; locations are shown in relation to plume position as of Day 1357. 
$10 \%$ sodium azide solution, for additional inhibition of microbial activity. Three of the six active microcosms were amended with $6.9 \mathrm{~mL}$ of a modified Bushnell Haas nutrient medium consisting of the following in distilled water $(/ \mathrm{L}): 1.0 \mathrm{~g} \mathrm{~K}_{2} \mathrm{HPO}_{4}, 1.0 \mathrm{~g} \mathrm{KH}_{2} \mathrm{PO}_{4}, 1.0 \mathrm{~g} \mathrm{NH}_{4} \mathrm{NO}_{3}, 0.2 \mathrm{~g}$ $\mathrm{MgSO}_{4} \cdot 7 \mathrm{H}_{2} \mathrm{O}, 0.02 \mathrm{~g} \mathrm{CaCl}_{2} \cdot 2 \mathrm{H} 2 \mathrm{O}, 0.005 \mathrm{~g} \mathrm{FeCl}_{3}$ (Mueller et al., 1991). The remaining three active microcosms were not amended with nutrients.

After completion, and prior to sample collection, the microcosms each contained approximately $660 \mathrm{~mL}$ of headspace air, which was intended to provide ample oxygen for maintenance of aerobic conditions. This was verified by headspace monitoring for oxygen over the experimental period which indicated that headspace oxygen concentration remained at, or near, atmospheric levels. The necks of three of the microcosms were broken by the pressure of the teflon stopcocks (two on day 0 and one on day 5). Contents of these microcosms were transferred, for the remainder of the experiment, to bottles similar in design but with only $800 \mathrm{~mL}$ capacity. The lower capacity did not have a significant effect on headspace oxygen content.

Microcosms were incubated at $10^{\circ} \mathrm{C}$ under static conditions and sampling was conducted at the following times after initial setup: 1, 5, 8, 15, 29 and 132 days. At each of these times, the microcosms were monitored for aqueous phase concentrations of selected creosote compounds and also for oxygen and carbon dioxide content of headspace gas. At the end of the experiment, total aerobic viable cell counts were determined for aquifer material from each microcosm to evaluate the effectiveness of sterilization procedures. The analytical method used for aqueous phase creosote compounds was identical to that used for plume sampling, discussed above. Headspace gas concentrations were analyzed with a gas partitioner equipped with a thermal conductivity detector. Total plate counts were determined by standard preparation techniques and were incubated under aerobic conditions, at 22-23 degrees $C$ for 14 days. Additional details on microcosm setup, headspace analysis and plate count analysis are provided in Appendix 10.

Results

Early and final concentrations of selected creosote compounds are shown in Table 4.1 and detailed results are provided in Appendix 10. Early concentrations were comparable between the control and the active microcosms and were therefore not appreciably affected by the sterilization procedure. Comparison of control and active concentrations indicate that biodegradation of phenol, naphthalene, dibenzofuran, carbazole and 1-methylnaphthalene occurred without the addition of nutrients but that biodegradation proceeded to a lower end concentration when nutrients were added. M-xylene and phenanthrene do not show appreciable biodegradation without nutrient addition. However, with nutrient addition the former compound is nondetectable at the end of the experiment and the latter shows appreciable biotransformation. Time series plots of the microcosm data (Figure 4.2) indicate that the rate of biodegration is most rapid for naphthalene and carbazole, both with and without nutrient addition. This diagram also clearly shows biodegradation of all compounds in the microcosms amended with nutrients and recalcitrance of $m$-xylene and phenanthrene when nutrients were not added. 


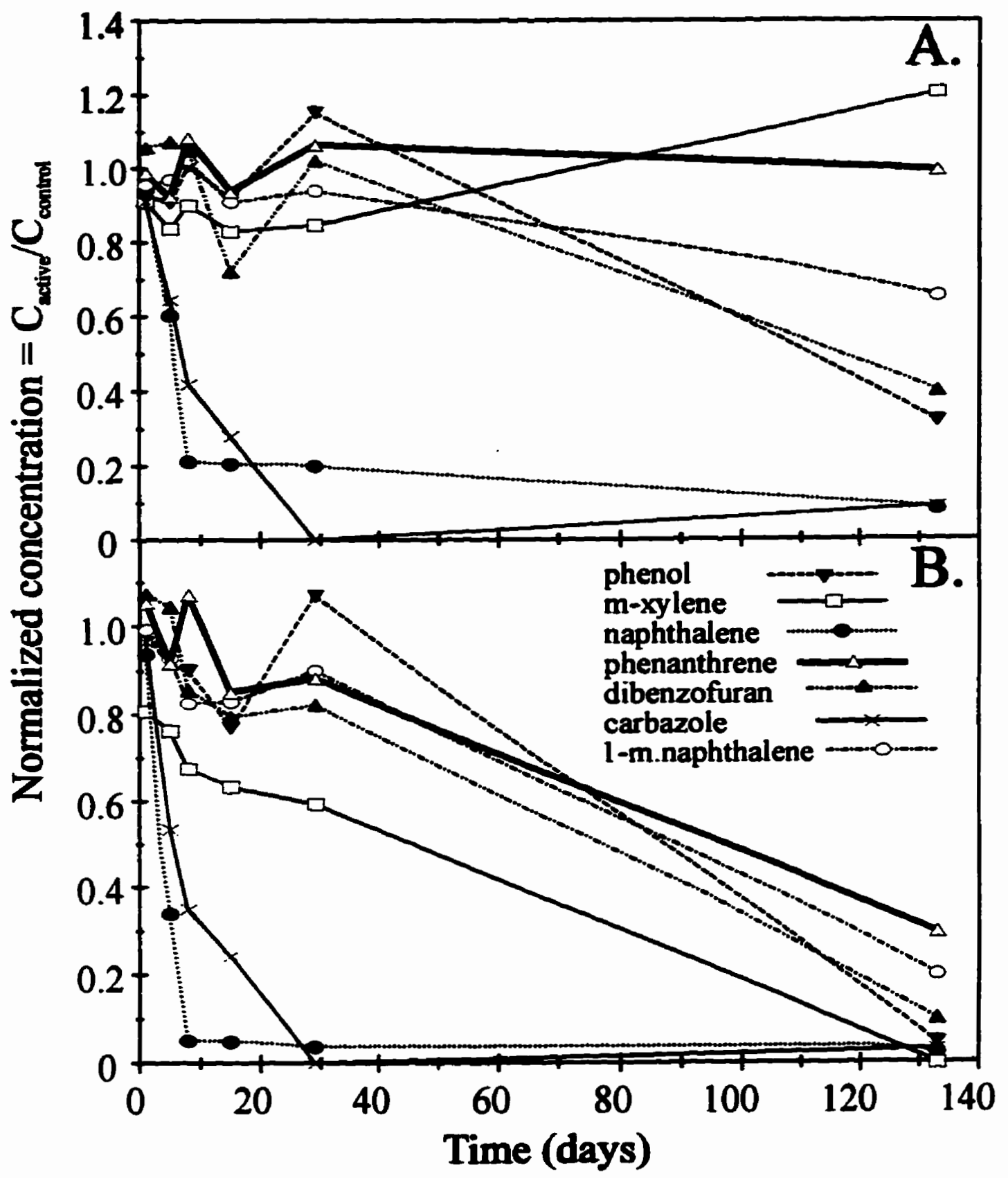

Figure 4.2. Plot of average normalized concentrations $(n=3)$ in aerobic microcosms without nutrient addition (A.) and with nutrients added (B.). 
Table 4.1. Mean aqueous concentrations ( $\mathrm{ppb} ; \mathrm{n}=3$ ) at the beginning and end of the microcosm experiment; microcosms were composed with Borden groundwater and aquifer sediments collected from within the dissolved creosote plume.

\begin{tabular}{lrrrrrr}
\hline Compound & \multicolumn{2}{c}{ Sterile Control } & \multicolumn{2}{c}{$\begin{array}{c}\text { Active / } \\
\text { no nutrients }\end{array}$} & \multicolumn{2}{c}{$\begin{array}{c}\text { Active / } \\
\text { nutrient addition }\end{array}$} \\
\cline { 2 - 7 } & Day I & Day 133 & Day I & Day 133 & Day l & Day 133 \\
\hline phenol & 6686 & 6785 & 6190 & 2161 & 6582 & 312 \\
m-xylene & 2897 & 1622 & 2636 & 1963 & 2344 & 0 \\
naphthalene & 3646 & 2841 & 3439 & 231 & 3419 & 73 \\
phenanthrene & 284 & 211 & 280 & 210 & 299 & 63 \\
dibenzofuran & 473 & 295 & 501 & 118 & 509 & 30 \\
carbazole & 451 & 291 & 412 & 26 & 436 & 9 \\
l-methylnaphthalene & 394 & 210 & 376 & 138 & 391 & 42 \\
\hline
\end{tabular}

Ample headspace oxygen persisted throughout the experiment to maintain aerobic conditions: from $22.34 \%$ to a minimum of $17.42 \%$, as shown in Appendix 10 . Headspace carbon dioxide results, summarized in Figure 4.3, indicate an accumulation of this gas in the active microcosms. These results can only be considered qualitatively since this gas may have originated from biodegradation of creosote compounds other than those analyzed. Also, the results have not been normalized for the variation in headspace size between the two different size microcosms. However, they represent additional qualitative evidence of microbial utilization of creosote compounds in the active microcosms.

Total plate count results for microcosm sand after the last set of aqueous and headspace samples are summarized in Table 4.2, and detailed results are provided in Appendix 10. These are based on an incubation time of 14 days but compare closely to another set that was incubated for 24 days. The sterilization and microbial inhibition measures used for the control microcosms were effective: plate counts for the control microcosms are low $(<10 \mathrm{CFU} / \mathrm{g})$ or nondetectable while the active microcosms are several orders of magnitude higher, ranging from $10^{6}$ to $10^{7} \mathrm{CFU} / \mathrm{g}$. This further supports the suggestion that the compound transformation determined for the active microcosms is due to microbial metabolism. 


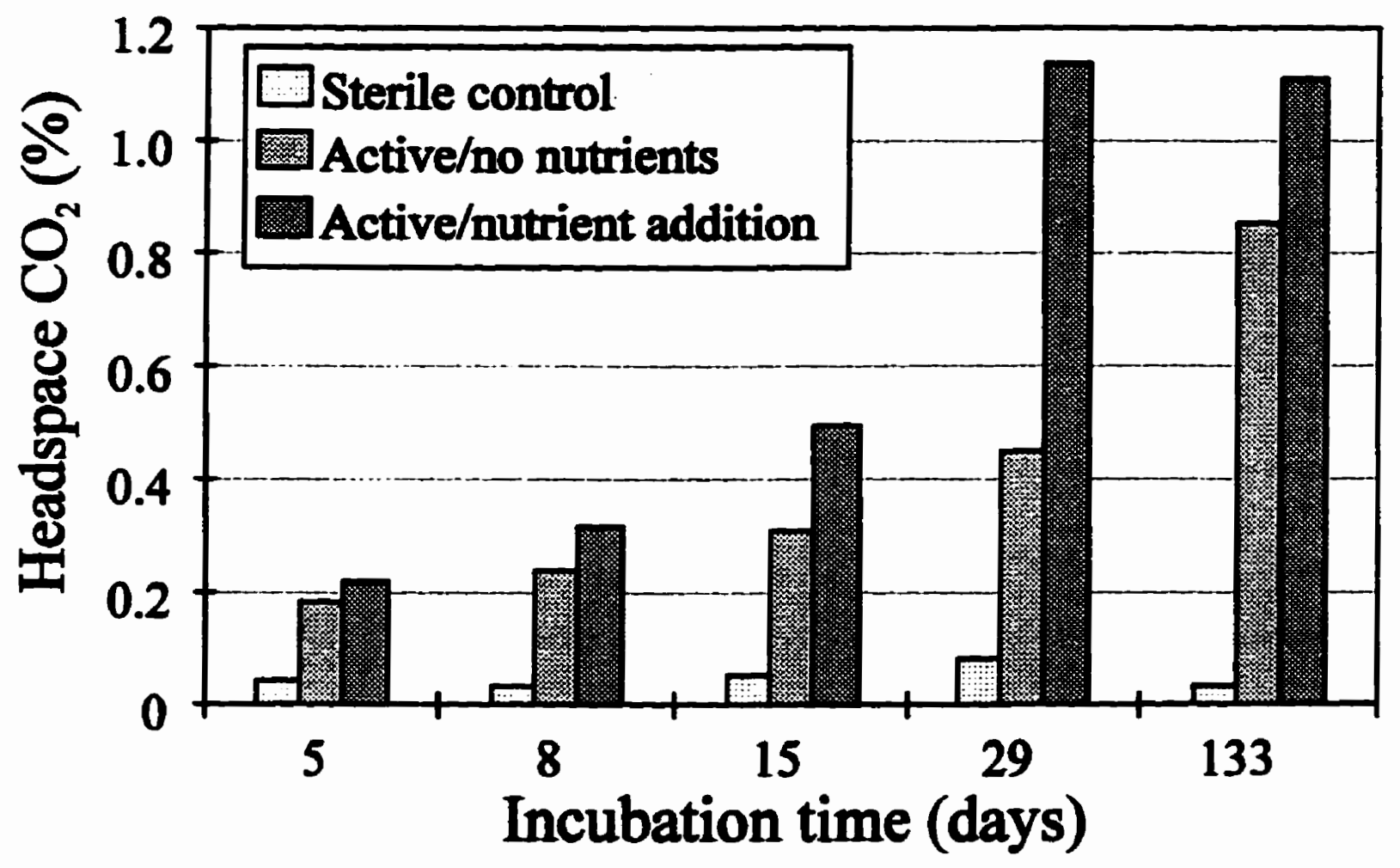

Figure 4.3. Bar graph of $\mathrm{CO}_{2}$ concentration in aerobic microcosms under three different treatments ( $n=3$ for each). 
Table 4.2. Results of plate count analysis for sand samples collected from microcosms at the end of the experiment.

\begin{tabular}{|c|c|c|c|}
\hline \multirow[t]{2}{*}{ Microcosm No. } & \multirow[t]{2}{*}{ Treatment } & \multicolumn{2}{|c|}{ Plate Count (CFU/g dwt; $n=3$ ) } \\
\hline & & Mean & S.D. \\
\hline 1 & Control & 0.0 & 0 \\
\hline 2 & “ & 8.3 & 12 \\
\hline 3 & cs & 8.3 & 5.9 \\
\hline 4 & Active/no nutrients & $2.4 \times 10^{7}$ & $9.2 \times 10^{6}$ \\
\hline 5 & c & $1.9 \times 10^{7}$ & $1.5 \times 10^{6}$ \\
\hline 6 & « & $2.1 \times 10^{7}$ & $2.1 \times 10^{6}$ \\
\hline 7 & Active/nutrient addition & $6.1 \times 10^{6}$ & $3.1 \times 10^{6}$ \\
\hline 8 & 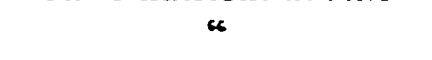 & $6.3 \times 10^{6}$ & $9.0 \times 10^{5}$ \\
\hline 9 & “6 & $6.1 \times 10^{6}$ & $3.5 \times 10^{5}$ \\
\hline
\end{tabular}

\section{PLUME REDOX CONDITIONS}

\section{Background}

The creosote plume was sampled for selected parameters related to utilization of electron acceptors (dissolved oxygen, $\mathrm{Fe}^{2+}, \mathrm{Mn}^{2+}, \mathrm{NO}_{3}{ }^{-}, \mathrm{NH}_{4}^{+}, \mathrm{SO}_{4}{ }^{2-}$, and methane) to evaluate for the type of changes that would be expected with biodegradation of creosote organics. In laboratory studies, microbial utilization of various aromatic, phenolic and heterocyclic compounds has been linked with dissolved oxygen utilization, denitrification (Major et al., 1988; Kuhn et al., 1988; Hutchins et al., 1991), iron reduction (Lovley et al., 1989) sulphate reduction (Haag et al., 1991; Edwards, et al., 1992) and methanogenesis (Godsy et al., 1992; Edwards and Grbic-Galic, 1994; Adrian and Suflita, 1994).

A range of field studies have presented geochemical evidence that is consistent with the laboratory results. Dissolved oxygen depletion has been documented for a number of petroleum hydrocarbon plumes (e.g., Chiang et al., 1989; Wilson et al., 1990; Barker et al., 1987) and has been related to biodegradation. In an extensive characterization an oil spill site at Bemidji, Minnesota, Bennet et al. (1993) delineated several characteristic redox zones. They determined that groundwater upgradient of the spill was oxygenated and that dissolved oxygen became slightly depressed as petroleum hydrocarbons began to mix with groundwater. As organic carton increased, groundwater became anoxic, methane occurred, dissolved iron and manganese increased and redox potentials indicated strongly reducing conditions. Similar redox trends were observed through intensive characterization studies at a creosote-contaminated site in Pensacola, Florida (Mattraw and Franks, 1986), and in a landfill leachate plume containing dissolved organics in Vejen, Denmark (Lyngkilde and Christensen, 1992b). 
Methods

Monitoring of redox-sensitive parameters was conducted at multilevel samplers within, and just outside the creosote plume, at the locations shown on Figure 4.1. Samples were collected within one week after the last two synoptic sampling events (1008 and 1357 days). Dissolved oxygen was analyzed in the field using CHEMetrics ampoules (CHEMetrics Incorporated) for the appropriate concentration range: 0-12 ppm, 0-1 ppm and 0-100 ppb. These $10 \mathrm{~mL}$ glass ampoules are under negative pressure and are partially filled with $4 \mathrm{~mL}$ of a Rhodazine-D solution. The tip of the ampoule is broken off when it is submerged in the sample, so that a portion of the sample is drawn into the ampoule and mixed with the solution. The colour change is proportional to dissolved oxygen content. White et al. (1990) described a down-hole technique whereby CHEMetrics ampoules were determined to be reliable for measuring low levels of dissolved oxygen in groundwater. Since the monitoring network for the current study consisted mostly of samplers constructed of small-diameter tubing (3.2 $\mathrm{mm} \mathrm{O.D.),} \mathrm{a} \mathrm{method} \mathrm{was} \mathrm{developed}$ for above ground measurement. A peristaltic pump was attached to a small ( $18 \mathrm{~mL})$ glass bottle through one port of a two-port suction head. The other port was attached to the sampler tube, causing groundwater to be drawn into the bottle when vacuum was applied. After purging of at least two tubing volumes, the suction head was removed from bottle and an ampoule was inserted. The tip of the ampoule was then broken off, drawing in a portion of the sample. Trials showed that it was possible to make repeatable readings of nondetectable dissolved oxygen by this method, using a low range ampoule $(0-100 \mathrm{ppb})$. This indicated that the method did not result in significant contamination of the sample with atmospheric oxygen.

Samples for the remaining inorganic parameters $\left(\mathrm{Fe}^{2+}, \mathrm{Mn}^{2+}, \mathrm{NO}_{3}^{-}, \mathrm{NH}_{4}^{+}\right.$and $\mathrm{SO}_{4}{ }^{2-}$ ) were recovered in a manner similar to that used for dissolved oxygen. Groundwater was pumped into a $60 \mathrm{~mL}$ glass bottle using a suction head and a peristaltic pump prior to splitting into two subsamples. The first subsample, for anion analysis, was poured directly into a $20 \mathrm{~mL}$ plastic sample bottle. The second, for analysis of cations, was drawn into a plastic syringe and then filtered into a $20 \mathrm{~mL}$ plastic bottle using a $0.2 \mu \mathrm{m}$ syringe filter, and then acidified with reagent grade $\mathrm{H}_{2} \mathrm{SO}_{4}$. Analysis for chloride was conducted as a safeguard against mistakenly identifying leachate samples as either background groundwater (no creosote compounds) or creosote plume water (reducing conditions). The leachate plume underlies the creosote plume (Figure 2.2) and is known to be relatively elevated in the chloride and sulphate (Nicholson et al., 1983).

Methane samples were recovered by a technique similar to the other types of samples: a suction head connected to a peristaltic pump. However, in the case of methane an $18 \mathrm{~mL}$ glass bottle was used and a crimp-top cap was applied directly to the bottle after purging. An effort was made to leave no headspace in the bottle but small bubbles were occasionally present. For analysis, a 15 $\mathrm{mL}$ aliquot was drawn from the sample bottle into a $30 \mathrm{~mL}$ glass syringe, followed by $15 \mathrm{~mL}$ of air. The syringe was then shaken and allowed to equilibrate for three hours. A 5-6 mL aliquot of the gas phase was then injected into a gas chromatograph equipped for flame ionization detection. Additional details on sampling methodology for redox-related parameters is provided in Appendix 11. 
Results

Data for selected redox-sensitive parameters and chloride are summarized in Table 4.3 and provided in detail in Appendix 11. Dissolved oxygen concentration averaged 0.13 within the creosote plume and $2.47 \mathrm{mg} / \mathrm{L}$ in background samples. A summary of statistical comparisons is provided in Table 4.4 and indicates that this difference was statistically significant and supports the expectation that dissolved oxygen has been consumed within the plume through the biodegradation of plume organics. Distributions of dissolved oxygen along two cross-sections are shown in Figure 4.4. The results shown in this diagram are associated with synoptic monitoring of dissolved creosote compounds conducted at day 1358 and the outline of the organics plume is indicated for comparison. The two cross-sections show that dissolved oxygen concentrations measured within the plume were less than or equal to $0.6 \mathrm{mg} / \mathrm{L}$, while concentrations outside generally ranged from 1.0 to $4.0 \mathrm{mg} / \mathrm{L}$. An area of depressed dissolved oxygen concentration was evident in the lower levels of samplers 6-N2 through 6-N5 and is considered to be attributable to influence from the underlying anaerobic landfill leachate plume.

Table 4.3. Summary of data for selected redox and geochemical indicator parameters; all concentrations are in $\mathrm{mg} / \mathrm{L}$.

\begin{tabular}{lcccccccccc}
\hline Parameter & \multicolumn{3}{c}{ Creosote Plume } & \multicolumn{3}{c}{ Background } & \multicolumn{3}{c}{$\begin{array}{c}\text { Landfill Leachate } \\
\text { Plume }\end{array}$} \\
\cline { 2 - 11 } & mean & n & s.d. & mean & n & s.d. & mean & n & s.d. \\
\hline Dissolved $\mathbf{O}_{2}$ & 0.13 & 63 & 0.16 & 2.47 & 18 & 0.99 & 0.12 & 12 & 0.20 \\
$\mathrm{Fe}^{2-}$ & 0.20 & 53 & 0.97 & 0.03 & 5 & 0.03 & 0.63 & 14 & 1.04 \\
$\mathrm{Mn}^{2+}$ & 0.13 & 53 & 0.16 & $<0.05$ & 5 & 0 & 0.024 & 14 & 0.04 \\
$\mathrm{NO}_{3}^{-}$ & 1.51 & 53 & 4.69 & 2.35 & 5 & 3.08 & 5.97 & 14 & 12.70 \\
$\mathrm{NH}_{4}^{-}$ & 0.21 & 53 & 0.25 & 0.62 & 5 & 0.42 & 0.22 & 14 & 0.22 \\
$\mathrm{SO}_{4}^{2-}$ & 11.57 & 53 & 4.19 & 14.1 & 5 & 3.79 & 46.23 & 14 & 30.37 \\
Methane $^{2-}$ & 0.036 & 53 & 0.104 & 0.001 & 2 & 0.001 & 0.034 & 13 & 0.050 \\
$\mathrm{Cl}^{-}$ & 1.93 & 14 & 0.52 & 4.04 & 3 & 3.13 & 26.75 & 6 & 10.96 \\
\hline
\end{tabular}

Note: nondetectable concentrations were handled as "zeroes" in calculations.

If biodegradation proceeds in the oxygen depleted portion of the plume then changes would be expected in the concentration of alternate electron acceptors. Indeed, a decrease in average nitrate concentration was noted from background groundwater to the plume, which would be consistent with nitrate utilization. However, both $\mathrm{NO}_{3}{ }^{-}$and $\mathrm{NH}_{4}^{+}$, which comprise a redox couple, displayed a high degree of variability and there was no significant difference between the plume and background averages. Behaviour of other redox-sensitive parameters was more consistent with expected trends. Average concentrations of reduced iron and manganese were significantly different between plume and background samples, with the largest occurring within the plume. Average sulphate concentration decreased significantly from $14.1 \mathrm{mg} / \mathrm{L}$ in background 


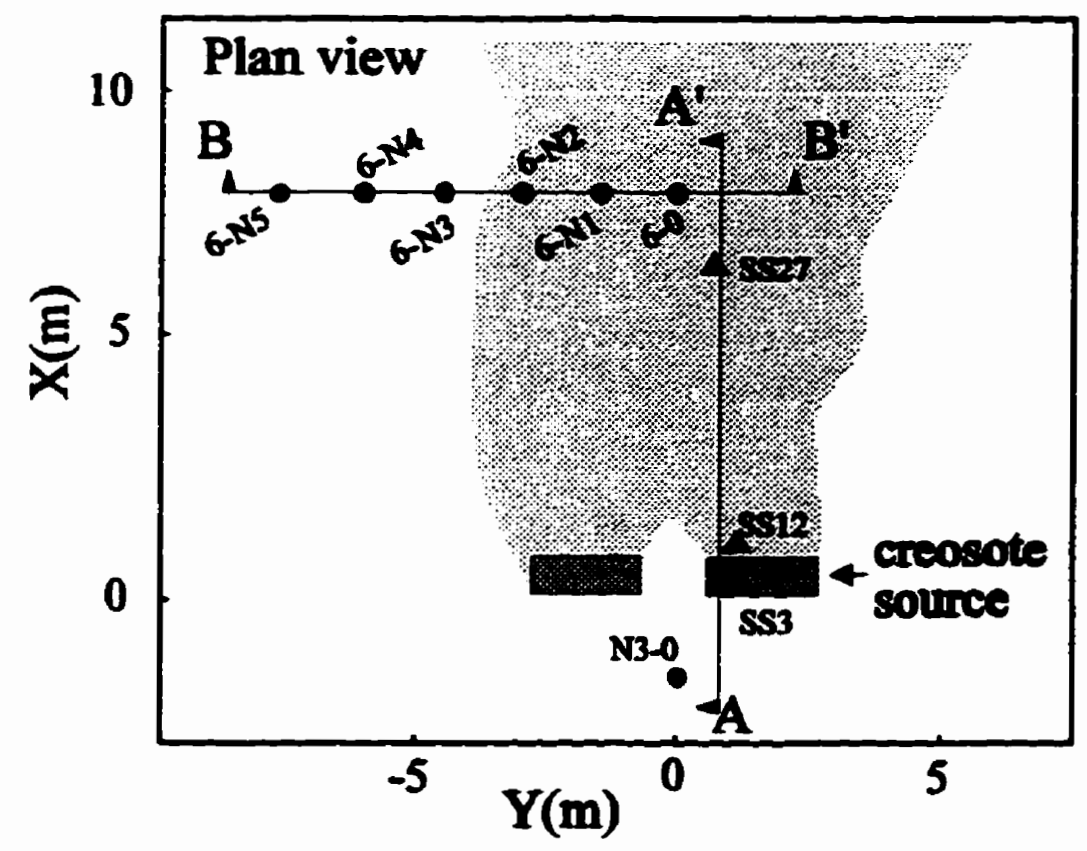

Multi-level samplers used for dissolved oxygen monitoring:

- Tefion

- Strinless steel

Extent of dissolved organies plume on Day 1358

is Dissolved oxygen

03 sampling point and concentration (mg/L)
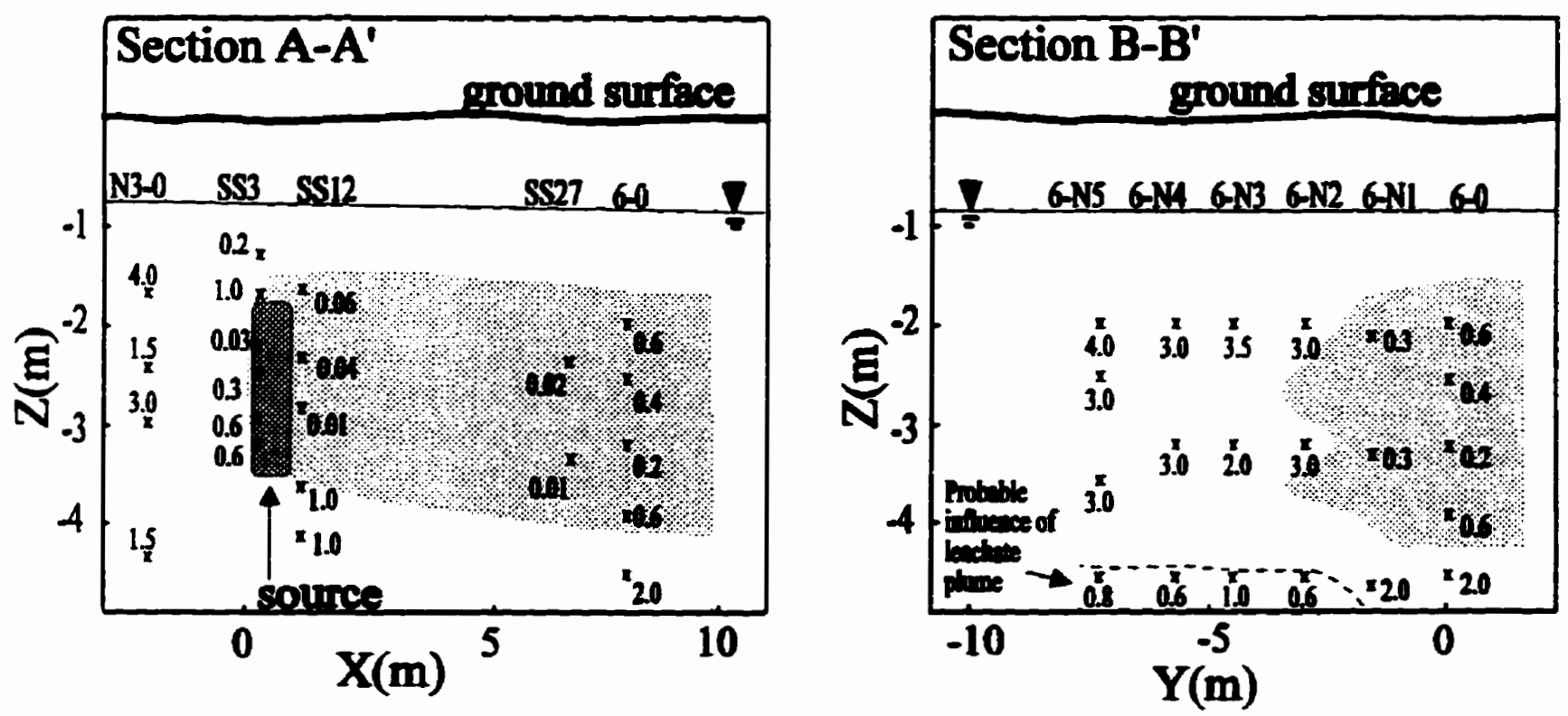

Figure 4.4. Plan showing dissolved oxygen monitoring locations along cross-sections oriented parallel to groundwater flow (A-A') and perpendicular (B-B'); cross-sections show distribution of dissolved oxygen relative to the dissolved creosote plume as of Day 1357. 
groundwater to $11.6 \mathrm{mg} / \mathrm{L}$ in the plume. A small but significant increase in average methane from $0.001 \mathrm{mg} / \mathrm{L}$ outside the plume to $0.036 \mathrm{mg} / \mathrm{L}$ inside is an indication of a minor degree of methanogenic activity within the plume. Results of samples from the landfill leachate plume are also summarized in Table 4.3 and, similar to the organics plume, they are indicative of conditions more reducing than background groundwater. All these samples were obtained from depths of $z=-4.2 \mathrm{~m}$ or greater, which also supports the suggestion that they are more typical of leachate chemistry.

Table 4.4. Results of mean concentration comparison ( $t$-test) for samples collected from within the dissolved creosote plume and background samples collected outside the plume.

\begin{tabular}{lcc}
\hline Parameter & Significant difference at 95\% level & Direction of difference \\
\hline Dissolved $\mathrm{O}_{2}$ & Yes & Background $>$ Plume \\
$\mathrm{Fe}^{2+}$ & Yes & Plume $>$ Background \\
$\mathrm{Mn}^{2+}$ & Yes & Plume $>$ Background \\
$\mathrm{NO}_{3}^{-}$ & No & Not Applicable \\
$\mathrm{NH}_{4}^{+}$ & No & Not Applicable \\
$\mathrm{SO}_{4}^{2-}$ & Yes & Background $>$ Plume \\
$\mathrm{Methane}^{2-}$ & Yes & Plume $>$ Background \\
$\mathrm{Cl}^{-}$ & No & Not Applicable \\
\hline
\end{tabular}

\section{MICROBIOLOGICAL INDICATORS}

\section{Background}

Aquifer samples were collected from inside and outside the creosote plume to evaluate for selected microbial parameters that might be expected to change as a result of plume biodegradation. Several field studies have identified changes in characteristics of microbial populations that have been linked to the introduction of organic compounds to groundwater. In a study conducted at a wood preserving plant in Pensacola, Florida, Godsy et al. (1992) determined that populations of methanogenic bacteria were approximately two orders of magnitude more numerous in pore water and aquifer samples from contaminated areas of the site than in uncontaminated areas. Elevated concentrations of methane within the contaminated areas and the observations of methanogenesis in laboratory microcosms composed with contaminated aquifer material, were evidence that methanogenic microorganisms played a role in the final fate of the contaminants. Madsen et al. (1991) determined that microorganisms from within plume associated with buried coal tar waste were capable of mineralizing naphthalene and phenanthrene over a three week period while those in pristine samples did not. They also determined that protozoa were more abundant within the plume and attributed this to increase in bacterial numbers due to contaminant utilization, and subsequent protozoan grazing on bacteria. 
In the current study, microbial characteristics were compared on the basis of phospholipid fatty acids (PLFA), a component of intact cell membranes. A number of researchers have contributed to the development and testing of methods that relate the quantity of PLFA in sediment samples to microbial biomass (e.g., White et al., 1979; Guckert et al., 1985; Balkwill et al., 1988; Ringelberg et al., 1989) and detailed analytical methods are provided by Dobbs and Findlay (1993). Extensions of this technique have been reviewed by Tunlid and White (1992) and have been used to relate the relative proportions of groups of PLFA compounds to microbial community structure and metabolic status. The summation of the ratio between certain trans and cis PLFAs $(16: 1 \omega 7 \mathrm{t} / 16: 1 \omega 7 \mathrm{c}$ and $18: 1 \omega 7 \mathrm{t} / 18: 1 \omega 7 \mathrm{c})$ has been related to changes in cell membranes of gram negative bacteria in response to environmental stresses. In general, larger values are indicative of toxicity or starvation effects. Similarly, gram negative bacteria convert certain monoenoic PLFAs to cyclopropyl fatty acids, as growth slows from a log to a stationary

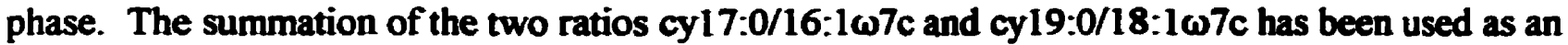
indicator of this process, with larger values indicative of a tendency towards stationary growth.

\section{Methods}

Aquifer cores for analysis of microbial indicator parameters were collected at the four locations shown in Figure 4.1. Coring was done with the wireline method described by Zapico et al. (1987) using five centimeter diameter core barrels rinsed with methanol and $\mathrm{MilliQ} \mathrm{H}_{2} \mathrm{O}$ prior to use. After collection, the cores were split lengthwise and placed in an aseptic hood where subsamples were collected from aquifer material in the central part of the core, not in contact with the core barrel. The core from outside the plume $(\mathrm{C} 1)$ and one from within the plume (C2) were subsampled from 1.4 to $2.6 \mathrm{~m}$ depth, at $20 \mathrm{~cm}$ intervals. The sample from the top of the plume core was at or near the top of the plume and the bottom sample was situated near the vertical centre of the plume. The other two plume cores ( $\mathrm{C} 3$ and $\mathrm{C4}$ ) which were both collected within $40 \mathrm{~cm}$ of $\mathrm{C} 2$, were subsampled near the top, middle and bottom of the cored interval (1.4, 2.0 and $2.6 \mathrm{~m}$ depth, respectively). All samples were analyzed for PLFA by the method presented by Dobbs and Findlay (1993). In addition, aliquots of selected samples were analyzed for total plate counts by standard preparation techniques and incubation under aerobic conditions for $\mathbf{4 0}$ days at $10^{\circ} \mathrm{C}$. Additional details of PLFA and plate count analyses are provided in Appendix 12.

\section{Results}

PLFA and plate count results are summarized graphically in Figure 4.5 and detailed results are provided in Appendix 12. Comparison of PLFA concentrations indicate that microbial biomass is greater within the plume than in background aquifer material, presumably due to greater microbial growth because of the large organic carbon source that the plume represents. PLFA data from both within and outside the plume do not show a strong trend with respect to depth. However, plate counts, expressed in the form of CFU/g dry weight aquifer material, decrease sharply with depth for the cores collected within the plume. Although plate counts were not determined for 

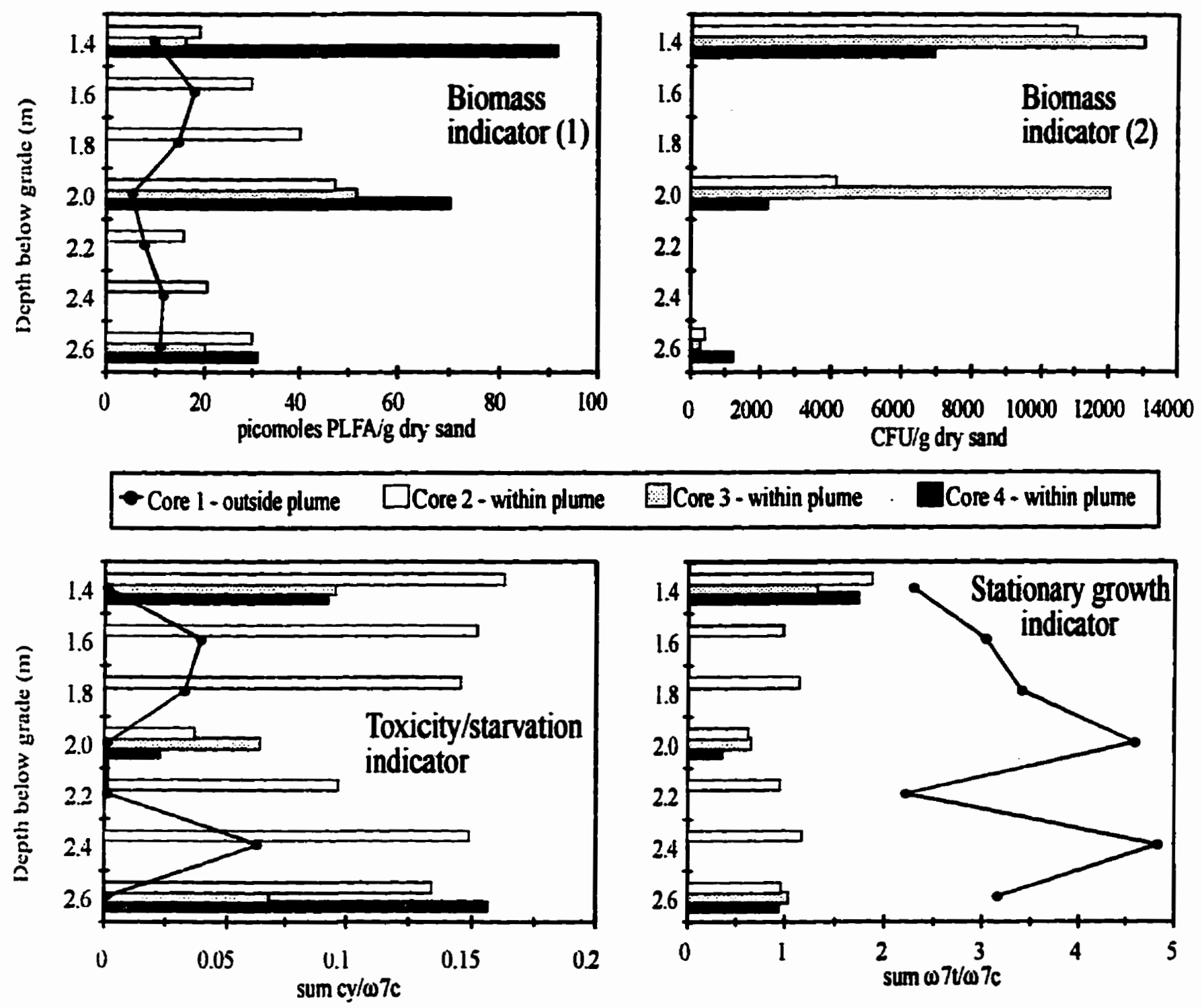

$\square$ Core 3 - within plume . Core 4 - within plume

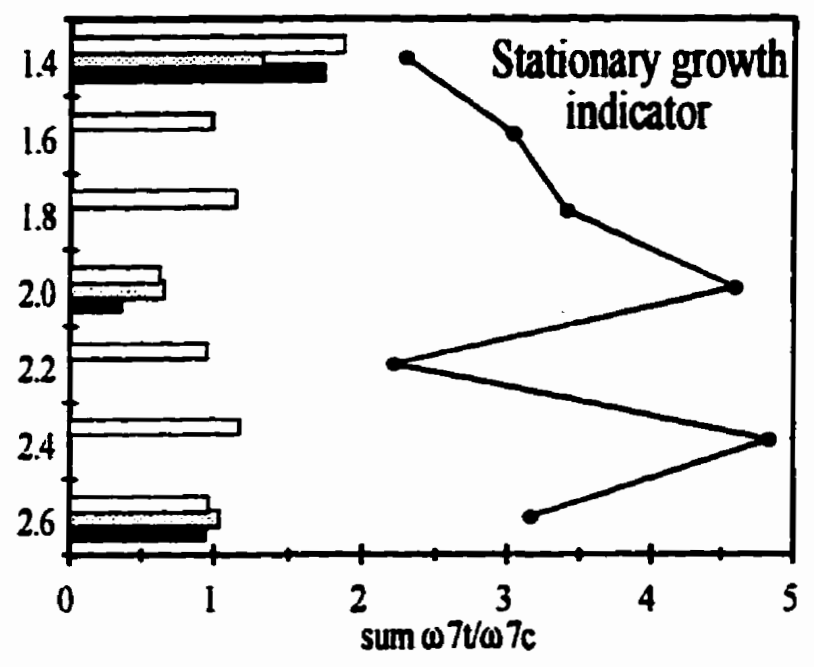

Figure 4.5. Indicators of microbial numbers and status in aquifer material collected from within and outside the dissolved creosote plume; "Total Biomass" has been evaluated by the concentration of PLFA (1) and by total plate count (2); the "Toxicity or starvation" indicator is determined as the sum of the ratio between certain groups of fatty acids $(16: 1 \omega 7 \mathrm{t} / 16: 1 \omega 7 \mathrm{c}+18: 1 \omega 7 \mathrm{t} / 18: 1 \omega 7 \mathrm{c})$; indication of microbial conformity to a "Stationary growth" phase is indicated by the sum of another pair of ratio (cyl7:0/16:107e + cy19:0/18:107c). 
background samples, an extensive evaluation of microbial characteristics in uncontaminated zones of the aquifer at a nearby location by Barbaro et al. (1994) generally showed the same trend of decreasing plate counts with depth. These researchers suggested that the trend was related to a similar trend in dissolved oxygen, which increased closer to the water table. Since the plate count analyses by Barbaro et al (1994) and that conducted for the current study were performed under aerobic conditions, they have provided preferential detection of aerobic microorganisms. Consequently, while the PLFA data from the current study indicated an increase in microbial biomass due to the presence of the plume, plate count data may be more affected by the trend in dissolved oxygen, which generally decreases with depth both inside and outside the plume. The PLFA results indicated that the decrease in aerobic microbes was generally accompanied by an increase in anaerobic microbes, resulting in no strong trends in biomass with depth.

Figure 4.5 also shows results for the PLFA ratio used as an indicators of microbial toxicity/starvation and stationary growth. Results for the former provide evidence that microorganisms within the plume are generally under more environmental stress than those in background core. This could be due to a degree of toxicity associated with plume compounds or may be indicative of an imbalance between organic sources and nutrients within the plume. The ratio used to indicate the tendency towards stationary growth are distinctly larger in the background core: evidence that the status of the Gram negative community tends towards stationary growth phase outside the plume and log growth within the plume. This is consistent with a larger turnover rate within the plume due to biodegradation of plume compounds and with the larger biomass, as indicated by total PLFA concentration.

\section{ORGANIC METABOLITES}

\section{Background}

Groundwater in the vicinity of the plume was evaluated for selected organic acids that might be expected to be formed through microbially mediated oxidation of plume compounds. These intermediate products, often termed "metabolites", are formed as biodegradation of the parent compound proceeds towards complete mineralization. Several studies have documented the groundwater occurrence of organic acids and other intermediate products associated with biodegradation of plumes from sources such as petroleum fuels, coal tar and creosote. Cozzarelli et al. (1995) detected a wide range of aliphatic and aromatic acids associated with a plume from a gasoline spill in Galloway Township, New Jersey. Through associated microcosm work, these researchers concluded that two of the simpler aromatic acids (benzoic and phenylacetic acid) were susceptible to further oxidization under nitrate reducing conditions while those with two or more methyl groups were resistant. Barcelona et al. (1995) detected aliphatic and aromatic acids in groundwater as transformation products at several petroleum hydrocarbon-contaminated sites, through a reconnaissance level survey. The authors suggested these acids were present in large enough concentrations to significantly influence geochemical processes, such as mineral solubility and trace metal availability, that may influence subsequent biodegradation. Aliphatic and aromatic 
acids were also detected in association with the Pensacola creosote plume (Goerlitz et al., 1985). Quinolinone, another metabolite, was detected in both the Pensacola creosote plume and in work previously reported for the current site (Fowler et al., 1994). This compound is a ketone of the $\mathrm{N}$-heterocyclic compound quinoline, which is a significant constituent in creosote. Bennet et al. (1986) determined that quinolinone was produced as a metabolite of quinoline by aerobic microorganisms from the Pensacola site. At the Bemidji site, Cozzarelli et al. (1994) attributed the observed accumulation of organic acids in groundwater to biodegradation of aromatic parent compounds in the anoxic portion of the plume. The authors compared the Bemidji plume to those at Halloway and Pensacola, concluding that depletion of electron acceptors through microbially mediated oxidation of parent aromatics allowed the buildup of organic acids.

\section{Methods}

Groundwater samples for aromatic acids were collected 1486 days after source emplacement, at the five multilevel samplers shown in Figure 4.1. Two or more levels were sampled at some locations, for a total of nine samples. Collection, extraction and analysis were conducted according to procedures provided by Barcelona et al. (1995). In addition, aliquots of each sample were analyzed for selected creosote compounds, according to the method described in Chapter 2. Creosote compounds and aromatic acids included in the analytical suite are listed in Table 4.5.

Table 4.5. List of aromatic acids and creosote compounds analyzed to evaluate for the presence organic metabolites.

\begin{tabular}{ll}
\hline Creosote compounds (25) & Aromatic acids (11) \\
\hline m-xylene, phenol, naphthalene, phenanthrene, & benzoic acid, 2-methylbenzoic acid, \\
dibenzofuran, carbazole, 1-methylnaphthalene, & methyl salicylate, 3-methylbenzoic acid, \\
o-cresol, p+m-cresol, 2,6-dimethylphenol, & 4-methylbenzoic acid, \\
2,4+2,5-dimethylphenol, 2,3-dimethylphenol, & 2,6-dimethylbenzoic acid, \\
3,5-dimethylphenol, indole+2-methylnaphthalene, & 2,5-dimethylbenzoic acid, \\
biphenyl, acenaphthylene, & $2,3+3,5$-dimethylbenzoic acid, \\
acenaphthene, dibenzofuran, & 2,4,6-trimethylbenzoic acid, \\
fluorene, anthracene, fluoranthene, pyrene & 3,4-dimethylbenzoic acid \\
\hline
\end{tabular}

Results

A summary of results for organic acid and selected redox-related parameters is provided in Table 4.6 and complete acids results are given in Appendix 13. Samples from N3-0 were collected upgradient of the creosote source and were intended to provide data representative of background conditions. For the most part, results indicate that this location is not affected by the plume: creosote compounds and $\mathrm{Fe}^{2+}$ were not detected, dissolved oxygen was greater than $1 \mathrm{mg} / \mathrm{L}$, and 
$\mathrm{SO}_{4}{ }^{2-}$ was relatively elevated. However, the presence of relatively low, but detectable, concentrations of aromatic acids at this location was unexpected. These acids may be due to a widespread low level presence of aromatic acids in background groundwater or may be due to inadvertent contamination of samples after collection.

Table 4.6. Results of groundwater samples used to evaluate for the presence of creosote plume metabolites: aromatic acids, creosote compounds and selected redox indicators.

\begin{tabular}{ccccccc}
\hline $\begin{array}{c}\text { Multilevel } \\
\text { sampler }\end{array}$ & $\begin{array}{c}\text { Sample } \\
\text { point } \\
\text { depth } \\
(\mathbf{m})\end{array}$ & $\begin{array}{c}\text { Total } \\
\text { aromatic } \\
\text { acids } \\
(\mathrm{ppb})\end{array}$ & $\begin{array}{c}\text { Total } \\
\text { creosote } \\
\text { compounds } \\
\text { (ppb) }\end{array}$ & $\begin{array}{c}\text { Dissolved } \\
\text { Oxygen } \\
\text { (ppm) }\end{array}$ & $\begin{array}{c}\text { Fe }^{2+} \\
\text { (ppm) }\end{array}$ & $\begin{array}{c}\text { SO4 }^{2-} \\
\text { (ppm) }\end{array}$ \\
\hline N3-0 & 1.8 & 42 & 0 & $>1$ & $<0.05$ & 15.4 \\
N3-0 & 3.0 & 278 & 0 & $>1$ & $<0.05$ & 14.1 \\
SS12 & 2.93 & 149 & 11834 & $<0.01$ & 0.06 & 9.29 \\
SS27 & 2.4 & 753 & 5535 & 0.02 & 0.12 & 11.0 \\
SS27 & 3.3 & 18312 & 1528 & 0.01 & 0.42 & 13.1 \\
$9-1$ & 1.8 & 128 & 0 & 0.4 & $<0.05$ & 15.7 \\
$9-1$ & 2.4 & 2694 & 3724 & 0.5 & 0.19 & 12.9 \\
$9-1$ & 3.6 & 356 & 11508 & 0.5 & 0.24 & 10.6 \\
$18-1$ & 3.2 & 1509 & 6050 & 0.4 & 0.08 & 10.8 \\
\hline
\end{tabular}

Data for field blank samples are not available for further evaluation, due to a laboratory accident. However, the absence of detectable organic acids in a laboratory procedure blank indicates that contamination did not occur during extraction and analysis. For the purpose of interpreting the organic acids data set, it has been assumed that concentrations up to the maximum detected in the upgradient monitoring points (278 ppb) are due to processes unrelated to creosote compounds. Total aromatic acids detected on the shallow level of 9-1, located $14.8 \mathrm{~m}$ downgradient of the source, were below this concentration, indicating that this point is probably in the upper periphery of the plume. The lack of detectable creosote compounds and $\mathrm{Fe}^{2+}$, as well as relatively elevated $\mathrm{SO}_{4}{ }^{2-}$, are further evidence that this location is above the plume, while the depressed dissolved oxygen concentration $(0.4 \mathrm{mg} / \mathrm{L})$ shows some degree of plume influence.

The aromatic acids concentration at SS12, located immediately downgradient of the source, is also within the background range, providing evidence that the higher concentrations detected in other plume samples originated from biodegradation of plume compounds and not from the original creosote. If the aromatic acids originated from the creosote source, relatively high concentrations would be expected at SS12, since it contained the highest concentrations of creosote compounds. It might be concluded, from the absence of elevated aromatic acids at SS12, that biodegradation had not occurred by the time the plume migrated to this near-source point. However, the relative depletion of both dissolved oxygen $(<0.01 \mathrm{mg} / \mathrm{L})$ and $\mathrm{SO}_{4}{ }^{2-}$ 
$(9.29 \mathrm{mg} / \mathrm{L})$ as well as the slightly elevated $\mathrm{Fe}^{2+}$ concentration $(0.06 \mathrm{mg} / \mathrm{L})$ indicated significant biodegradation. If this is the case, then aromatic acids have been metabolized concomitantly with the parent organics, and have therefore not accumulated.

Five other samples have been drawn from within in the plume, as indicated by detectable creosote compounds, and all of these show distinct evidence biodegradation and buildup of aromatic acids. Organic acids concentrations within this group range from $356 \mathrm{ppb}$ at $9-1$ ( $3.6 \mathrm{~m}$ depth) to a maximum of $18,312 \mathrm{ppb}$ at SS27 (3.3 m depth). The concentration at the latter location is more than an order of magnitude larger than that of total detected creosote compounds at the same location and almost twice that of detected creosote compounds at SS12, the most highly concentrated area of the plume. These samples also show concentrations of redox-sensitive parameters that are also consistent with plume biodegradation: dissolved oxygen and $\mathrm{SO}_{4}{ }^{2-}$ concentrations were below background and $\mathrm{Fe}^{2+}$ was somewhat elevated, as indicated by comparison with N3-0. 


\section{CHAPTER 5 \\ CONCEPTUAL MODEL OF PLUME BEHAVIOUR}

\section{INTRODUCTION}

A common trait shared by many non-halogenated hydrocarbons (e.g., PAHs, monoaromatics, phenolics and heterocyclics, as well as aliphatics and alicyclics compounds) is that they are susceptible to oxidation by microbial action. Furthermore, the tendency for biodegradation generally increases with the oxidizing potential of the aqueous setting. A generalized conceptual model for natural attenuation of these type of compounds in groundwater has gradually developed over the past two decades. Conclusions pertaining to the natural evolution of redox zones in groundwater (Champ et al., 1979) have been extended to anthropogenic sources of biodegradable carbon, in the form of landfills (Baedecker and Back, 1979a; Baedecker and Back, 1979b; Nicholson et al., 1983). With mounting evidence showing the environmental biodegradability of non-halogenated hydrocarbons (Alexander, 1985; Atlas, 1981), the concept of redox zonation was extended to groundwater plumes evolving from petroleum products (e.g., Bennet et al., 1993) and other organic mixtures containing similar ranges of compounds (e.g., Mattraw and Franks, 1986). Furthermore, it has been recognized that biodegradation of these chemicals is favoured under more oxidizing conditions (Lee et al., 1988; Grbic Galic, 1990a; MacFarland and Sims, 1991). Also contributing to this framework was the development and application of mathematical modelling (Borden and Bedient, 1986; MacQuarrie et al., 1990), which elucidated the important role of dispersion in plume biodegradation.

The purpose of this chapter is to evaluate certain aspects of the conceptual model for plumes that are susceptible to microbially-mediated oxidation, in the context of the study plume. The general effect of biodegradation on plume behaviour is demonstrated by comparison with a non-degrading solute. Then the distribution and utilization of electron acceptors are compared to the conceptual model. In doing so, the relative importance of aerobic biodegradation versus biodegradation utilizing alternate electron acceptors was evaluated. In addition, total electron acceptor utilization was balanced against observed organics mass loss to test the applicability of the conceptual model, based on this assumption. The discrepancy in this balance is discussed in terms of organic intermediates (metabolites). 


\section{CONCEPTUAL MODEL}

Figure 5.1(a) illustrates the development of an organic plume from a source that dissolves gradually into groundwater moving through the source. An expression that is often applied to describe the movement of a solute in groundwater is the advection-dispersion equation (Bear, 1972):

$$
D_{i j} \frac{\partial^{2} C}{\partial x^{2}}-v_{i} \frac{\partial C}{\partial x}-R \frac{\partial C}{\partial t}=0
$$

where $C=$ concentration $\left(M / L^{3}\right) ; D=$ the dispersion coefficient $\left(L^{2} / T\right) ; v=$ average linear groundwater velocity $(\mathrm{L} / \mathrm{T})$; and $t=$ time. Since the types of solutes that are of interest in this paper typically possess a degree of hydrophobicity, this equation is shown in a form that can represent the effect of sorption ideality on transport, as provided by the last term. In this term, $R=$ a retardation factor $=I+(\rho / \eta) K_{d}$ where $\rho=$ bulk density of the porous medium $\left(\mathrm{M} / \mathrm{L}^{3}\right) ; \eta=$ aquifer porosity (dimensionless); and $K_{d}=$ aqueous/solids partitioning coefficient $\left(\mathrm{L}^{3} / \mathrm{M}\right)$. Sorption will tend to decrease the apparent velocity of a solute in groundwater in proportion to $R$ such that:

$R=v_{g w^{\prime}} v_{s}$

where $v_{g w}=$ average linear groundwater velocity and $v_{s}=$ apparent solute velocity. In the absence of mass transformation, the outward development of the plume would continue under the influence of advection and dispersion. The latter process would cause a gradual decrease in concentration along the flowpath over relatively long migration distances. With mass transformation associated with biodegradation, decreases in concentrations occur over a shorter distance. Biodegradation also results in depletion of the most readily utilized electron acceptors within the plume. Since these most favourable electron acceptors are still present in groundwater just ouside the plume, the degree to which they participate in subsequent plume biodegradation becomes controlled by the rate at which mixing occurs on the plume periphery, due not only to outward flux of the plume but also to inward flux of the electron acceptor. This dispersive process is represented by the dispersion tensor term in Equation (5.1). In the three dimensional case, where flow is entirely in the $x$-direction, this simplifies to three dispersion coefficients:

$D_{L}=\alpha_{L} v+D^{*}$

$D_{T H}=a_{T H} v+D^{*}$

$D_{\pi v}=\alpha_{\pi v} v+D^{*}$

Where the subscripts $L, T H$ and $T V$ indicate parameters that apply in directions parallel to flow, transverse to flow in the horizontal $(x-y)$ plane and transverse to flow in the vertical $(x-z)$ plane, respectively; $\alpha=$ dispersivity of the porous medium $(L)$; and $D^{*}=$ effective diffusion coefficient of the solute $\left(\mathrm{L}^{2} / \mathrm{T}\right)$. 
a. Plume growth: dissolution, atvection, dispersion

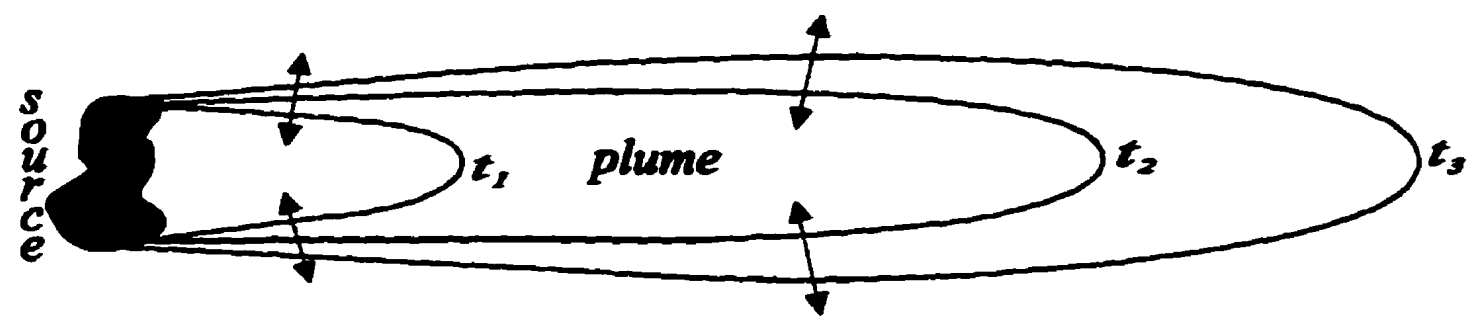

b. Redox zonation of primary electron soceptors

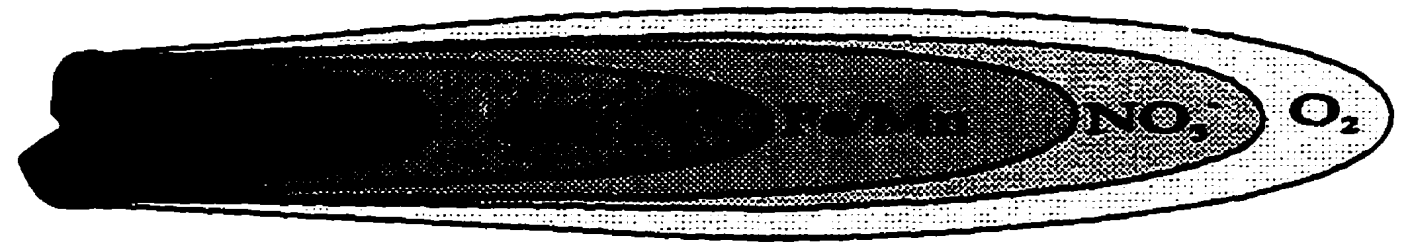

c. Zonation of biodegradation potential

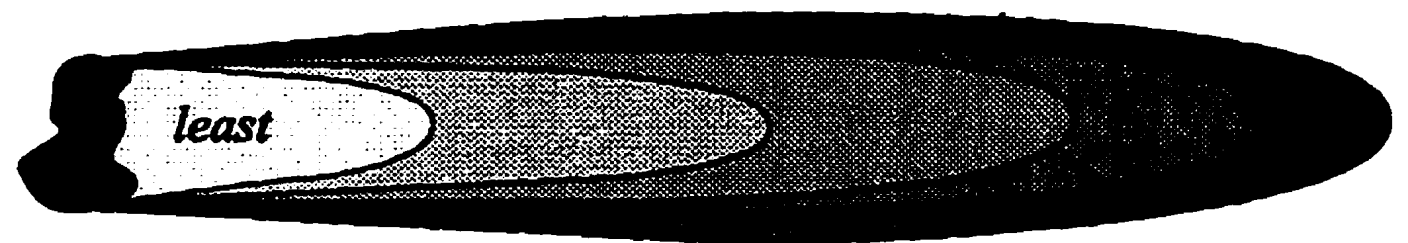

d. Steady state phume configuration

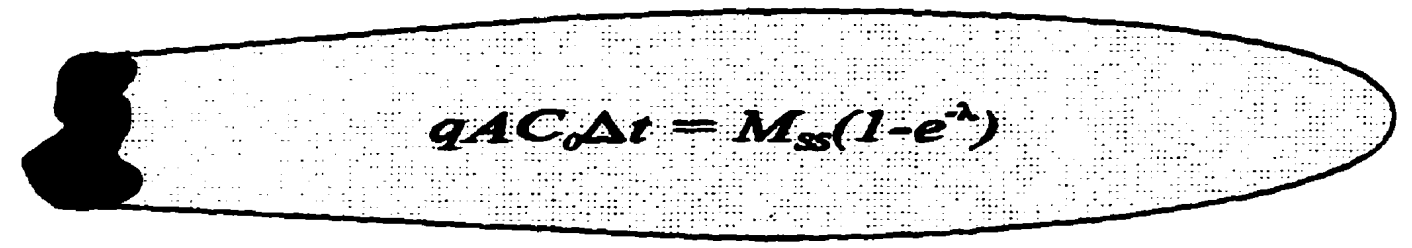

Figure 5.1. Plan-view illustration of the conceptual model for natural attenuation of aerobically biodegradable organics in groundwater: a) plume expands through dissolution from an organic source followed by advection, outward dispersive mixing of plume organics and inward dispersive mixing of mobile electron acceptors; b) preferential utilization of electron acceptors causes redox zonation; c) general dependance of biodegradation on redox condition causes zonation of biodegradation potential; d) plume stabilizes as the overall rate of organic mass transformation approaches the rate of source dissolution. 
This peripheral mixing leads to a zonation of redox conditions according to the sequence of reactions proposed by Stumm and Morgan (1970) and shown in Table 5.1, where depletion of an electron acceptor leads to a decrease in oxidizing potential, leading to utilization of the next available electron acceptor in the sequence. In a dynamic groundwater system containing an biodegradable organic plume, these reactions lead to the zonation shown in Figure 5.1(b). These processes have been discussed in a hydrogeological context by Baedecker and Back (1979b), in conjunction with the development of redox zones downgradient of a landfill. A more recent landfill study (Lyngkilde and Christensen, 1992b) provided detailed delineation of these characteristic redox zones. Zonation has also been shown to develop in biodegradable plumes from other organic sources, such as creosote (Mattraw and Franks, 1986) and petroleum hydrocarbons (Bennet et al., 1993).

Table 5.1. Standard Reduction Potentials and Free Energy Changes for Half Cell Reactions of primary electron acceptors.

\begin{tabular}{|c|c|c|c|}
\hline $\begin{array}{l}\text { Sequence }{ }^{1} \text { of } \\
\text { Electron Acceptors }\end{array}$ & Half cell reaction ${ }^{2}$ & $\underset{(V)}{E_{H}^{0}(W)^{2,3}}$ & $\begin{array}{c}\Delta G_{H}^{0}(W)^{24} \\
(\mathrm{~kJ} / \mathrm{mol})\end{array}$ \\
\hline Oxygen & $\mathrm{O}_{2}(\mathrm{~g})+4 \mathrm{H}^{-}+4 \mathrm{e}^{-}=2 \mathrm{H}_{2} \mathrm{O}$ & +0.81 & -78.3 \\
\hline Nitrate & $2 \mathrm{NO}_{3}^{-}+12 \mathrm{H}^{+}+10 \mathrm{e}^{-}=\mathrm{N}_{2}(\mathrm{~g})+6 \mathrm{H}_{2} \mathrm{O}$ & +0.74 & -71.4 \\
\hline Manganese & $\begin{array}{l}\mathrm{MnO}_{2}(\mathrm{~s})+\mathrm{HCO}_{3}^{-}\left(10^{-3} \mathrm{M}\right)+3 \mathrm{H}^{+}+2 \mathrm{e}^{-}= \\
\mathrm{MnCO}_{3}(\mathrm{~s})+2 \mathrm{H}_{2} \mathrm{O}\end{array}$ & +0.52 & -50.2 \\
\hline Iron & $\begin{array}{l}\mathrm{FeOOH}(\mathrm{s})+\mathrm{HCO}_{3}^{-}\left(10^{-3} \mathrm{M}\right)+2 \mathrm{H}^{+}+\mathrm{e}^{-}= \\
\mathrm{FeCO}_{3}(\mathrm{~s})+2 \mathrm{H}_{2} \mathrm{O}\end{array}$ & -0.05 & +4.6 \\
\hline Sulphate & $\mathrm{SO}_{4}^{-2}+9 \mathrm{H}^{+}+8 \mathrm{e}^{-}=\mathrm{HS}^{-}+4 \mathrm{H}_{2} \mathrm{O}$ & -0.22 & +21.3 \\
\hline Carbon dioxide & $\mathrm{CO}_{2}(\mathrm{~g})+8 \mathrm{H}^{+}+8 \mathrm{e}^{-}=\mathrm{CH}_{4}(\mathrm{~g})+2 \mathrm{H}_{2} \mathrm{O}$ & -0.25 & +23.5 \\
\hline
\end{tabular}

Electron acceptors utilized earlier in the redox sequence are generally the most energetic, as indicated by the free energy changes for electron acceptors provided in Table 5.1. Consequently, the development of redox zonation also causes zonation in the potential for biodegradation. As shown in Figure 5.1(c), the most rapid rates are expected at the plume periphery, where dissolved organic compounds mix with oxygenated background groundwater. Rates are expected to increase inward, to a minimum in the methanogenic core. 
Several field studies of dispersion in sand and gravel aquifers (e.g., Sudicky et al, 1983;

Garabedian et al., 1991; van der Kamp et al., 1994) have shown that $\alpha_{L}$ is large relative to $\alpha_{T H}$ and that the latter is large relative to $\alpha_{T *}$. Consequently, for biodegradable organic compounds, the potential for biodegradation (per unit area) is expected to be largest at the plume front, moderate on the sides of the plume and lowest at the upper and lower boundaries. It therefore follows that the relative importance of biodegradation at these three general plume locations (front, transverse horizontal periphery and transverse vertical periphery) will depend on plume configuration and on aquifer dispersivities $\left(\alpha_{L}, \alpha_{T H}\right.$ and $\alpha_{T V}$, respectively).

An extension of this concept may apply if the plume appoaches steady state as shown in Figure 5.1(d), which is the condition where the mass input to the plume over a given time period $(\Delta t)$ is equal to the mass biodegraded in the plume:

$q A C_{0} \Delta t=M_{s s}\left(1-e^{-\lambda}\right)$

where $q=$ Darcy flux $(\mathrm{L} / \mathrm{T}) ; A=$ cross-sectional area of the source transverse to groundwater flow direction $\left(\mathrm{L}^{2}\right) ; C_{0}=$ source concentration $\left(\mathrm{M} / \mathrm{L}^{3}\right) ; M_{S S}=$ steady state plume mass $(\mathrm{M})$; and $\lambda$ =plume-scale decay constant $(1 / T)$. As steady state is approached, biodegradation in the front region of the plume is expected to decrease, since the forward edge has ceased to advance. At this point, the relative importance of biodegradation on the sides of the plume is expected to increase.

\section{RESULTS AND DISCUSSION}

\section{General Effect of Plume Biodegradation}

The code BIO3D was used to evaluate the behaviour of the plume on the basis of several simplifying assumptions. This numerical model is capable of simulating biodegradation limited by the concentration of a mobile electron acceptor and utilizing Monod kinetics. The original code was developed in 2-D by MacQuarrie et al. (1990) and later developed to 3-D by Frind et al. (1989). Some of the parameter values related to aquifer properties and the source zone used in the model are shown in Table 5.2. A sensitivity analysis showed that dispersion in the z-direction (vertical transverse) had only a minor effect on plume transformation, due to the relatively small value for $\alpha_{T v}$ in the Borden aquifer: $10^{-10} \mathrm{~m}$ according to Sudicky et al. (1983). This value is several orders of magnitude smaller than $\alpha_{T H}$ and is in the range of molecular diffusion. Consequently the model was configured in quasi-2D by setting up the grid with only one element in the $z$-direction, where $\Delta z$ was equal to the vertical thickness of the source $(1.7 \mathrm{~m})$. Additional details of this analysis are provided in Appendix 14. 
Table 5.2. Model input parameters

\begin{tabular}{lcc}
\hline \multicolumn{1}{c}{ Parameter } & Value & Source \\
\hline Porosity & 0.33 & Mackay et al. (1986) \\
Ground water velocity & $0.091 \mathrm{~m} /$ day & Mackay et al. (1986) \\
Longitudinal dispersivity & $0.36 \mathrm{~m}$ & Freyberg (1986) \\
Transverse horizontal & $0.039 \mathrm{~m}$ & Freyberg (1986) \\
dispersivity & & \\
Source concentration & $0.4 \mathrm{mg} / \mathrm{L}$ & this study \\
Source configuration & 2 lobes $\times 2 \times 1.7 \mathrm{~m}$ & this study \\
Background D.O. & $2.5 \mathrm{mg} / \mathrm{L}$ & this study \\
Source D.O. & $0 \mathrm{mg} / \mathrm{L}$ & this study \\
Retardation factor & 4.67 & this study \\
\hline
\end{tabular}

The modelling analysis described herein was targeted at dibenzofuran. Monitoring and mass balance calculations for this compound have shown that it essentially reached steady state by 626 days after source emplacement, as illustrated in Figure 2.8. This distinctive behaviour provides a convenient benchmark for comparison with model results. Figure 5.2(a) shows the observed configuration of the dibenzofuran plume at 626, 1008 and 1357 days, as indicated by the by the position of the $0.02 \mathrm{mg} / \mathrm{L}$ contour at each of these times. Model results for a compound with source and sorption characteristics similar to those of dibenzofuran, but undergoing no biodegradation, are shown in Figure 5.2(b). Comparison of these results show, as expected, that the modelled plume continued to expand throughout this period, consistent with the suggestion that the actual dibenzofuran plume had stabilized due to transformation of dibenzofuran.

Figure 5.2(c) shows results intended to provide a preliminary indication of the relative importance of aerobic respiration in dibenzofuran transformation. This analysis used identical parameter values to those used previously with the exception that biodegradation was included. Background dissolved oxygen was set at a value representative of the study site $(2.5 \mathrm{mg} / \mathrm{L})$ and kinetic terms were adjusted so that biodegradation was essentially instantaneous in the presence of oxygen. Dissolved oxygen in groundwater flowing from the source was set at zero, on the basis of field measurements that showed oxygen to be depleted at locations immediately downgradient of the source. A value of $\mathbf{2 . 5 7}$ was used for the stoichiometric ratio between oxygen and dibenzofuran, according to the reaction:

$\mathrm{C}_{12} \mathrm{H}_{8} \mathrm{O}+13.5 \mathrm{O}_{2}-12 \mathrm{CO}_{2}+4 \mathrm{H}_{2} \mathrm{O}$

which assumes complete mineralization of the organic compound. As shown in 5.2(c), the modelled location of the dibenzofuran plume at 1357 days was less extensive than the nonbiodegrading case but has migrated substantially further than the observed plume. The former observation was expected since 


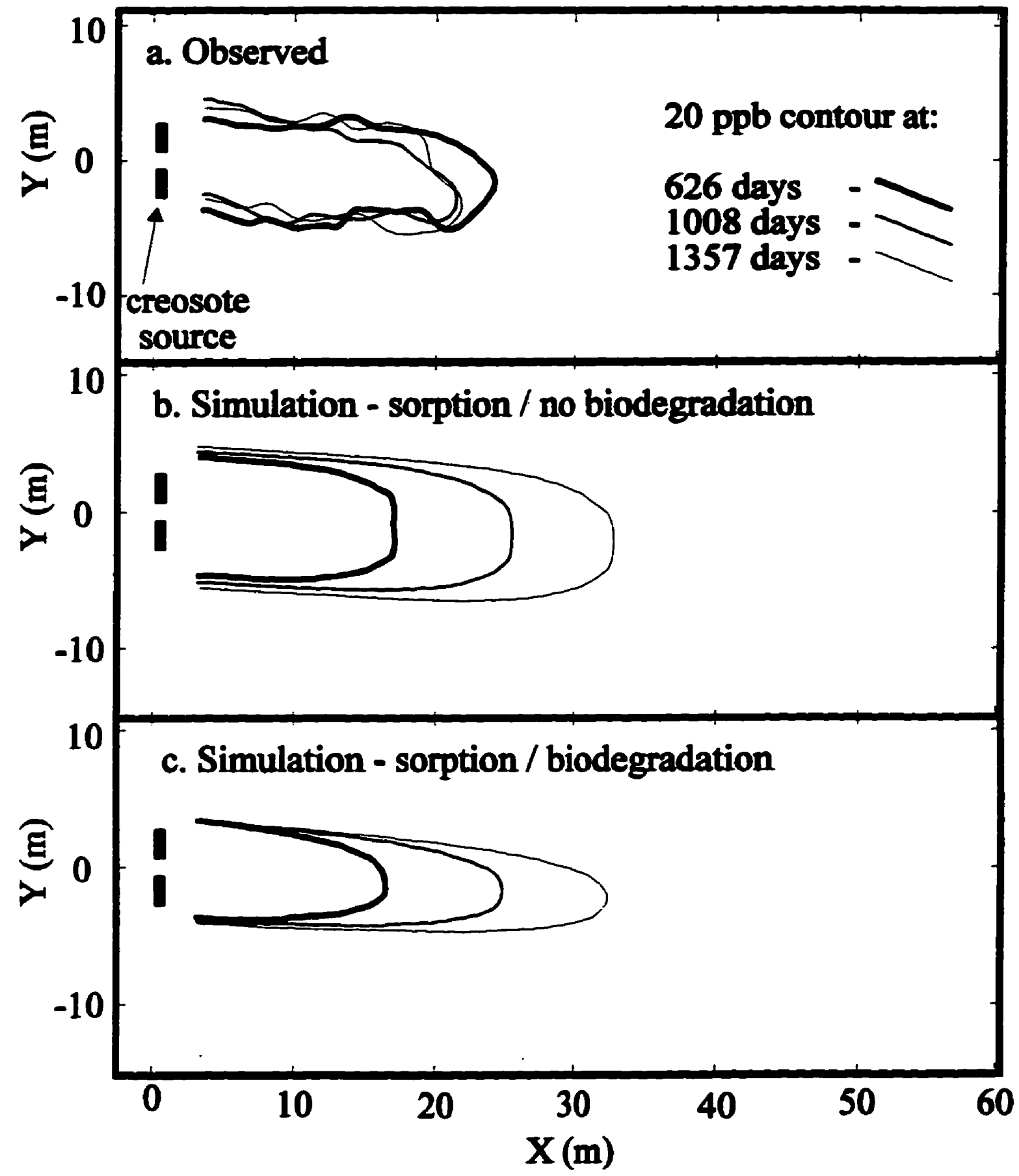

Figure 5.2. Configuration of the $20 \mathrm{ppb}$ contour for the dibenzofuran plume at 626,1008 and 1357 days according to plume monitoring (a.) and simulations with BIO3D (b. and c.). 
biodegradation tends to limit migration. However, the latter was more surprising since dibenzofuran makes up a relatively small proportion of total plume mass. As shown in Table 5.3, the mass of dibenzofuran in the plume ranged from one to two percent of total mass for the seven compounds represented in the table. Since the plume also contained compounds that are not represented in the table, the actual proportion of plume organics comprised of dibenzofuran was even smaller. Obviously, the exposure of dibenzofuran to oxygen under the modelled conditions was unrealistically high, yet the observed plume was still less extensive than predicted by the model. There are at least two potential explanations for this: 1) a significant portion of the biodegradation utilized other electron acceptors, 2) biodegradation did not proceed to complete mineralization so that the stoichiometric ratio used for dibenzofuran transformation is unrealistically large. These are discussed in the following.

Table 5.3. Masses of selected compounds in the emplaced creosote source plume; includes dissolved phase (from monitoring) and sorbed phase (estimated from $K_{d}$ 's).

\begin{tabular}{lccc}
\hline Compound & $\begin{array}{c}626 \\
\text { days }\end{array}$ & $\begin{array}{c}1008 \\
\text { days }\end{array}$ & $\begin{array}{c}1357 \\
\text { days }\end{array}$ \\
\hline$m$-xylene & 216.0 & 164.7 & 76.2 \\
phenol & trace & trace & trace \\
naphthalene & 732.9 & 957.2 & 1242.8 \\
phenanthrene & 0.359 & 5.54 & 3.16 \\
dibenzofuran & 21.72 & 22.04 & 22.56 \\
carbazole & 7.22 & 17.15 & 15.98 \\
l-methylnaphthalene & 21.99 & 27.33 & 40.93 \\
\hline
\end{tabular}

\section{Electron Acceptors}

A summary of data related to electron acceptors $\left(\mathrm{O}_{2}, \mathrm{NO}_{3}{ }^{-}\right.$and $\left.\mathrm{SO}_{4}{ }^{2-}\right)$ or reduced products $\left(\mathrm{Fe}^{2+}\right.$, $\mathrm{Mn}^{2+}$ and $\mathrm{CH}_{4}$ ) is shown in Figure 5.3 in the form of a comparison between groundwater inside and outside the plume. In general, concentrations changed in a manner that is consistent with the conceptual model of aerobic biodegradation on the periphery of the plume and more reducing conditions within the plume: $\mathrm{O}_{2}, \mathrm{NO}_{3}{ }^{-}$and $\mathrm{SO}_{4}{ }^{2-}$ decreased while $\mathrm{Fe}^{2+}, \mathrm{Mn}^{2+}$ and $\mathrm{CH}_{4}$ concentrations increased. This is evidence that alternate electron acceptors play a significant role in biodegradation of the plume.

An indication of electron acceptor distribution along a plume cross-section oriented approximately parallel to groundwater flow is shown in Figure 5.4. Although there are too few data points to provide detailed delineation, it is apparent that some redox zonation occurs but that well-defined zonation has not developed. Dissolved oxygen was depleted within the plume, although low concentrations persisted throughout. $\mathrm{NO}_{3}^{-}$was persistent at appreciable concentrations, even though evidence exist that less energetic oxidants were utilized: $\mathrm{Fe}^{2+}, \mathrm{Mn}^{2+}$ 


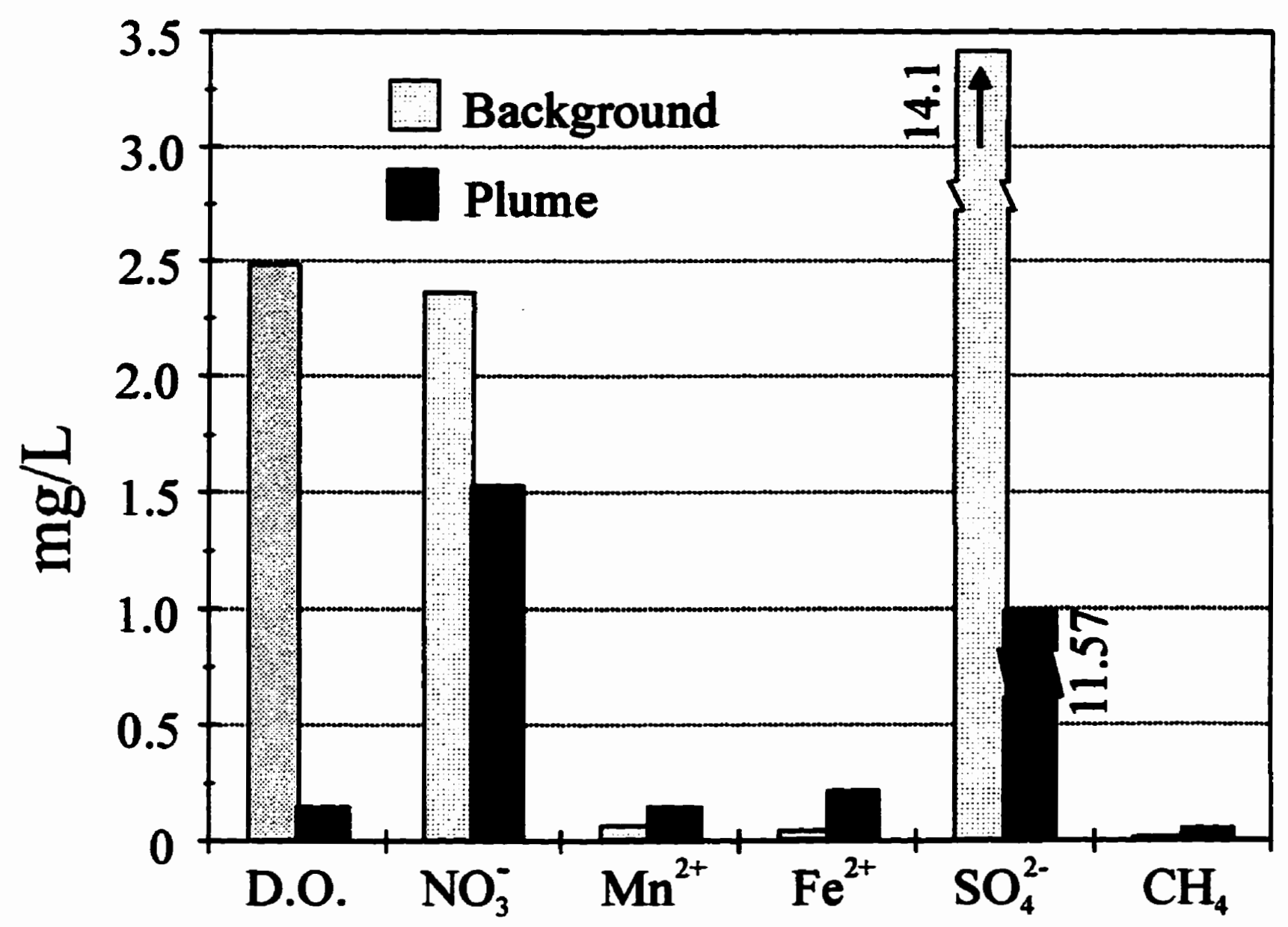

Figure 5.3. Average concentrations of redox-related parameters in background and plume groundwater; samples were collected following synoptic plume sampling at 626, 1008 and 1357 days after source emplacement. 

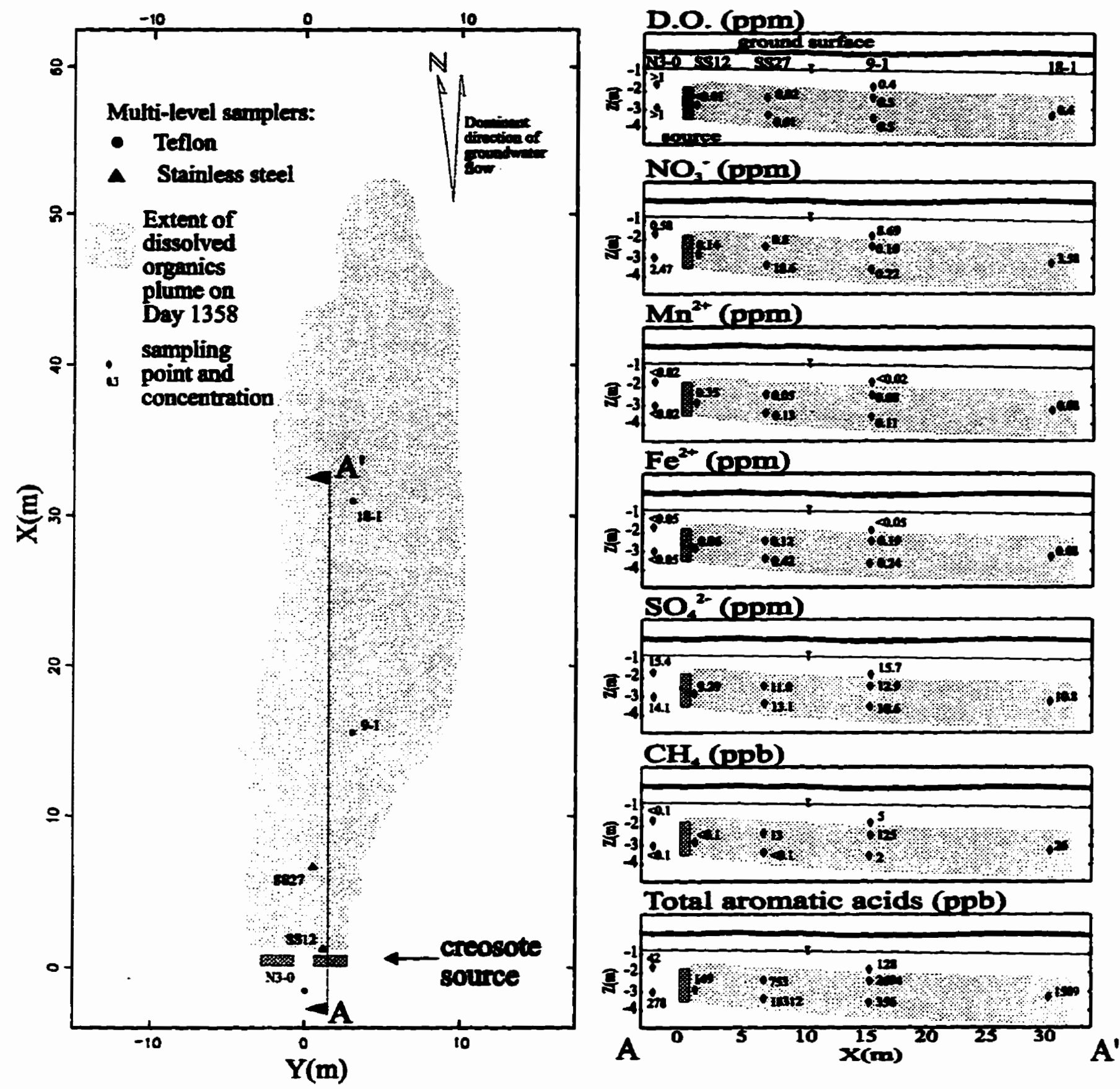

Figure 5.4. Distribution of oxidation reactants and products at 1460 days after source emplacement; plan view on left shows areal locations of selected multi-level samplers; cross-sections on right side show point sampling locations and associated concentrations. 
and $\mathrm{CH}_{4}$ have generally increased while $\mathrm{SO}_{4}{ }_{4}^{2-}$ decreased. In general, the distribution of electron acceptors indicated that plume biodegradation had not achieved equilibrium, with persistence of more energetic electron acceptors at locations where less energetic acceptors had apparently been utilized. This may be due to transient conditions with respect to flow direction as well as aquifer heterogeneities, which may cause mixing of electron acceptors, rather than sequential depletion, as would be expected along a consistent flow path. Evidence of substantial variability in groundwater flow direction was determined by Linderfelt (pers. comm) and is shown in Figure 5.5.

The overall significance of various electron acceptors in plume biodegradation was estimated by expressing concentration change as a stoichiometric potential for biodegradation. Table 5.4 shows the stoichiometric ratio between oxygen and six plume organics and illustrates the relatively small range in these values, from 2.57 to 3.24 . Ratios for other electron acceptors are not shown, but these generally show the same relative range in values, with respect to the six compounds shown, and other creosote compounds. On that basis, the values for dibenzofuran have been used as an approximation for all plume compounds; these values are shown in Table 5.5. The total mass of individual electron acceptors $\left(M_{O X}\right)$ involved in biodegradation over a given period of time $(t)$ was estimated according to:

$M_{O X}=\left(A_{\text {ALAX }}-A_{S R d}\right) q t \Delta C_{O X}$

where $A_{\text {MLAT }}=$ maximum plume cross-sectional area perpendicular to groundwater flow $\left(\mathrm{L}^{2}\right) ; A_{S R C}$ $=$ cross-sectional area of plume influent $\left(\mathrm{L}^{2}\right) ; q=$ Darcy flux $(\mathrm{L} / \mathrm{T}) ; \Delta C_{O X}=$ change in average concentration of oxidation reactant or product from background to plume $\left(M / L^{3}\right)$, as estimated from the average concentrations depicted in Figure 5.3. This approach asssumes that plume mixing does not occur substantially beyond the lateral boundaries of the plume: a reasonable assumption considering the small $\alpha_{T H}$ and $\alpha_{T V}$ values determined for this site. The expected loss of organics associated with the change in electron acceptor was calculated on the basis of stoichiometric ratios for dibenzofuran, and assumed complete mineralization, as per the reactions shown in Table 5.5 .

Table 5.4. Oxidation reactions involving oxygen and selected creosote compounds.

\begin{tabular}{lcc}
\hline Compound & Reaction & $\begin{array}{c}\text { Stoichiometric mass ratio } \\
\left(\mathrm{O}_{2} \text { :organic }\right)\end{array}$ \\
\hline m-xylene & $\mathrm{C}_{8} \mathrm{H}_{10}+10.5 \mathrm{O}_{2}-8 \mathrm{CO}_{2}+5 \mathrm{H}_{2} \mathrm{O}$ & 3.17 \\
naphthalene & $\mathrm{C}_{10} \mathrm{H}_{8}+13 \mathrm{O}_{2}-10 \mathrm{CO}_{2}+4 \mathrm{H}_{2} \mathrm{O}$ & 3.24 \\
dibenzofuran & $\mathrm{C}_{12} \mathrm{H}_{8} \mathrm{O}+13.5 \mathrm{O}_{2}-12 \mathrm{CO}_{2}+4 \mathrm{H}_{2} \mathrm{O}$ & 2.57 \\
phenanthrene & $\mathrm{C}_{14} \mathrm{H}_{10}+16.5 \mathrm{O}_{2}-14 \mathrm{CO}_{2}+5 \mathrm{H}_{2} \mathrm{O}$ & 2.96 \\
carbazole & $\mathrm{C}_{12} \mathrm{H}_{8} \mathrm{~N}+15.5 \mathrm{O}_{2}-12 \mathrm{CO}_{2}+4 \mathrm{H}_{2} \mathrm{O}+\mathrm{NO}_{3}^{-}$ & 2.97 \\
l-m.naphthalene & $\mathrm{C}_{11} \mathrm{H}_{10}+13.5 \mathrm{O}_{2}-11 \mathrm{CO}_{2}+5 \mathrm{H}_{2} \mathrm{O}$ & 3.04 \\
\hline
\end{tabular}




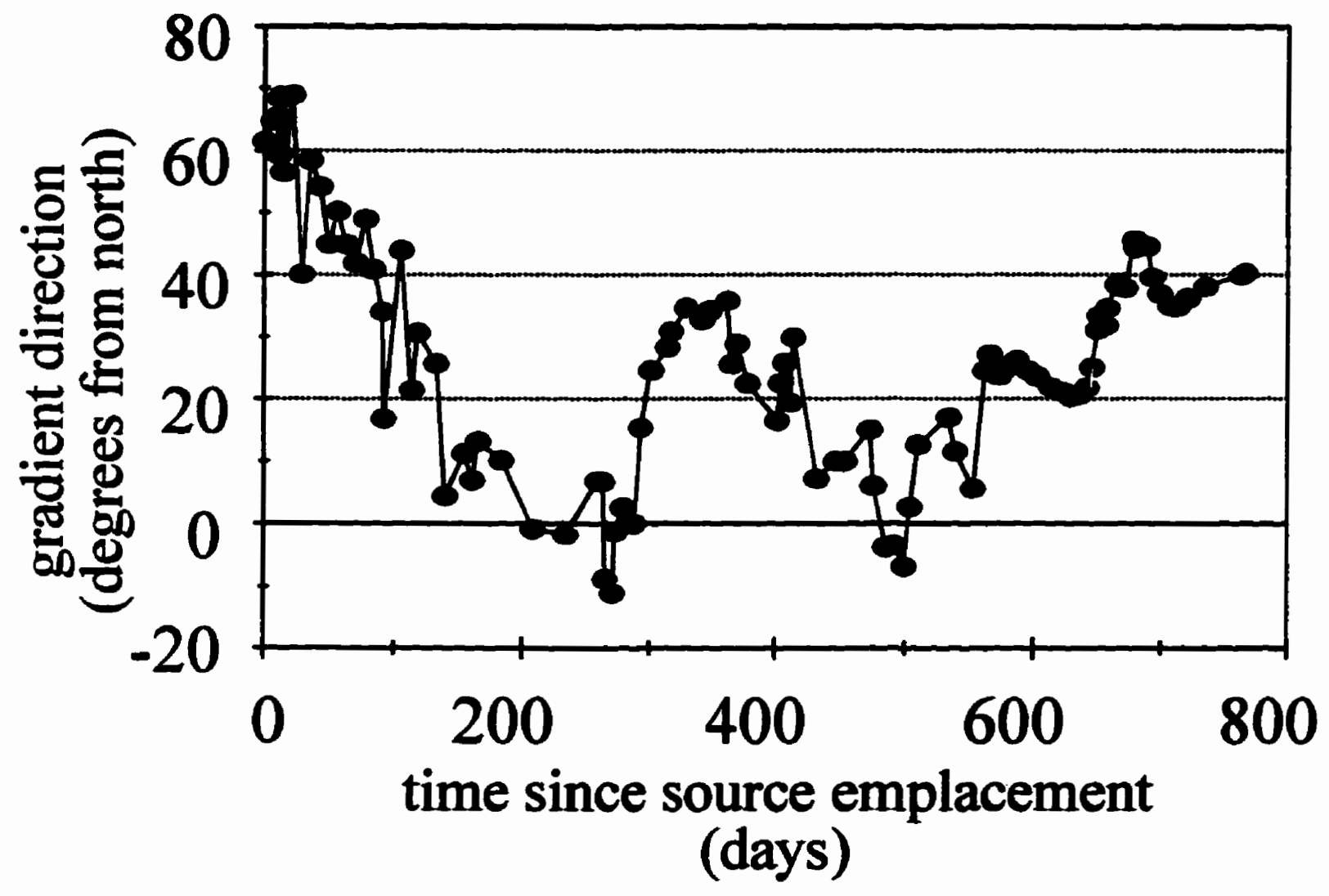

Figure 5.5. Variation in direction of groundwater hydraulic gradient at the emplaced creosote source site; data from Linderfelt (pers. comm.). 
Calculation results are summarized in Table 5.6. They indicate that $\mathrm{O}_{2}$ was the most important electron acceptor, accounting for just over half of the expected biodegradation, closely followed by $\mathrm{SO}_{4}^{2-}$ (approximately 40\%). Although average $\mathrm{NO}_{3}^{-}$concentration differed between the plume and background (Figure 5.3), associated biodegradation was not calculated, since the change was determined to be statistically insignificant (see Chapter 4). Biodegradation coupled to production of $\mathrm{Fe}^{2+}$ and $\mathrm{Mn}^{2+}$ was apparently minor in this plume, with each accounting for less than $1 \%$ of the total. Methanogenesis accounted for a slightly larger proportion, approximately $3 \%$.

Table 5.5. Overall oxidation reactions involving dibenzofuran and primary electron acceptors.

\begin{tabular}{lcc}
\hline $\begin{array}{l}\text { Process } \\
\text { (monitored parameter) }\end{array}$ & Overall reaction & $\begin{array}{c}\text { Stoichiometric } \\
\text { mass ratio } \\
\text { (parameter:organic) }\end{array}$ \\
\hline $\begin{array}{l}\text { aerobic respiration } \\
\left(\mathrm{O}_{2}\right)\end{array}$ & $\mathrm{C}_{12} \mathrm{H}_{8} \mathrm{O}+13.5 \mathrm{O}_{2}-12 \mathrm{CO}_{2}+4 \mathrm{H}_{2} \mathrm{O}$ & 2.57 \\
$\begin{array}{l}\text { denitrification } \\
\left.\left(\mathrm{NO}_{3}\right)^{-}\right) \\
\text {manganese reduction } \\
\left(\mathrm{Mn}^{2+}\right)\end{array}$ & $\mathrm{C}_{12} \mathrm{H}_{8} \mathrm{O}+10.8 \mathrm{NO}_{3}{ }^{-}+10.8 \mathrm{H}^{+}-12 \mathrm{CO}_{2}+4 \mathrm{H}_{2} \mathrm{O}+5.4 \mathrm{~N}_{2}$ & 3.98 \\
$\begin{array}{l}\text { iron reduction } \\
\left(\mathrm{Fe}^{2+}\right)\end{array}$ & $\mathrm{C}_{12} \mathrm{H}_{8} \mathrm{O}+27 \mathrm{MnO}_{2}+54 \mathrm{H}^{+}-12 \mathrm{CO}_{2}+31 \mathrm{H}_{2} \mathrm{O}+27 \mathrm{Mn}^{2+}$ & 8.82 \\
$\begin{array}{l}\text { sulphate reduction } \\
\left(\mathrm{SO}_{4}{ }^{2-}\right) \\
\text { methanogenesis } \\
\left(\mathrm{C} \mathrm{H}_{3}\right)\end{array}$ & $\mathrm{C}_{12} \mathrm{H}_{8} \mathrm{O}+54 \mathrm{Fe}(\mathrm{OH})_{3}+108 \mathrm{H}^{+}-12 \mathrm{CO}_{2}+139 \mathrm{H}_{2} \mathrm{O}+54 \mathrm{Fe}^{2+}$ & 17.93 \\
\hline
\end{tabular}

A comparison of the total stoichiometric change in electron acceptors vs. the total observed change in six plume compounds is provided in Table 5.6. Results for the plume compounds have been determined through detailed plume mass balance as discussed in Chapter 3 . Laboratory quantification for 19 additional creosote compounds (see Appendix 2) indicated that these six represented more than half of the plume mass. However, assuming that oxidation proceeds to complete mineralization, as per the reactions in Tables 5.4 and 5.5, then the change in electron acceptors accounted for only approximately one quarter of the mass transformation calculated for the six compounds. This discrepancy is even more significant considering that additional transformation of other compounds had probably also occurred. The most probable explanation for this discrepancy is that transformation does not necessarily proceed to complete mineralization but may result in production of intermediate products (metabolites). Consequently, compound disappearance requires lower than expected quantities of electron acceptors. The production of metabolites within the plume was discussed in Chapter 4 and is further discussed below, in the context of the conceptual model. 
Table 5.6. Balance of oxidation reactants or products against mass loss of creosote compounds; for period from 626 to 1357 days after source emplacement.

\begin{tabular}{|c|c|c|c|}
\hline $\begin{array}{l}\text { Oxidation } \\
\text { reactant } \\
\text { or product } \\
\end{array}$ & $\begin{array}{c}\text { Total estimated } \\
\text { change due to plume } \\
\text { (g) }\end{array}$ & $\begin{array}{l}\text { Associated change expected } \\
\text { in plume organics } \\
\text { (g) }\end{array}$ & $\begin{array}{l}\text { Observed loss of } 6 \\
\text { plume organics } \\
\text { (g) }\end{array}$ \\
\hline $\mathrm{O}_{2}$ & -477.7 & 185.9 & - \\
\hline $\mathrm{NO}_{3}^{-}$ & -172.0 & $\mathrm{na}^{2}$ & - \\
\hline $\mathbf{M n}^{2+}$ & +16.4 & 1.9 & - \\
\hline $\mathrm{Fe}^{2+}$ & +34.8 & 1.9 & - \\
\hline $\mathrm{SO}_{4}^{2-}$ & -516.5 & 133.8 & - \\
\hline $\mathrm{CH}_{4}$ & +7.2 & 11.3 & - \\
\hline see te & Total forivation & 334.8 & 1220.6 \\
\hline
\end{tabular}

Organic Metabolite Distributions

Metabolic oxidation of aromatic, heterocyclic and phenolic compounds is known to involve breakdown of the parent compound through sequences of partially-oxidized intermediate products (Grbic-Galic, 1989 and 1990a; Smith, 1990), sometimes called metabolites. A number of organic metabolites have been detected in the current study plume and were discussed in Chapter 4 . An indication of their distribution, as total aromatic acids, is shown in Figure 5.4. The extent to which the production of metabolites may affect electron acceptor utilization is difficult to quantify because a wide variety of these products may be produced, depending on redox condition and parent compound. In addition, little is known about their environmental persistence. However, the effect of metabolite production on electron acceptor requirement can be demonstrated in a qualitative sense by considering the transformation of naphthalene to salicylic acid, a pathway discussed by Smith (1990). Both parent and intermediate were detected in the study plume, although it is possible that the latter could also originate from other parent compounds. A possible overall reaction to describe this pathway under aerobic conditions is:

$$
\underset{\text { (naphthalenc) }}{\mathrm{C}_{10} \mathrm{H}_{8}}+5 \mathrm{O}_{2}-3 \mathrm{CO}_{2}+1 \mathrm{H}_{2} \mathrm{O}+\underset{\text { (salicylic acid) }}{\mathrm{C}_{7} \mathrm{H}_{6} \mathrm{O}_{3}}
$$

In this reaction, the stoichiometric ratio between $\mathrm{O}_{2}$ and naphthalene is 1.24 , as opposed to 3.24 in the reaction shown in Table 5.4, where naphthalene transformation proceeds to complete mineralization. This illustrates two important points with regard to organic compound biodegradation. First, the potential for parent compound transformation involving a given mass of electron acceptor may be substantially underestimated if complete mineralization is assumed. This 
does not contradict the concept of redox zonation as outlined in the conceptual model. However, it does imply that the redox zones of early electron acceptors $\left(\mathrm{O}_{2}\right.$ and $\left.\mathrm{NO}_{3}{ }^{-}\right)$may be larger than expected: the electron acceptors may penetrate further into the plume than expected, before they are depleted. Second, the production of persistent metabolites adds a degree of complication to predicting and modelling plume configuration as a function of biodegradation and is generally not accounted for in the conceptual model. 


\section{CHAPTER 6 SUMMARY AND CONCLUSIONS}

In general, trends in compound flux from the source were qualitatively consistent with predictions based on Raoult's law (Chapter 2). Selected organic compounds displayed an entire spectrum of plume behaviour. Phenol was completely dissolved from the source relatively early in the study and migrated downgradient as a discrete slug. Minimal detection of phenol in synoptic monitoring at 627 days indicated that this compound was substantially transformed. The $m$-xylene plume was observed to migrate to a maximum observed distance at 627 days and then to recede back toward the source as the rate of flux into the plume decreased to below the rate of plume transformation.

The phenanthrene plume was also observed to recede, although the flux of phenanthrene into the plume continued to increase up to the last synoptic monitoring event. Advancement of the carbazole plume was similar in that the rate of advancement had decreased, or possibly halted, in the most recent snapshot. However, in the case of carbazole, mass flux from the source also decreased, probably in response to source depletion with respect to this compound.

The plume of dibenzofuran remained relatively constant in extent during the last two years of monitoring. A relatively constant rate of dibenzofuran dissolution from the source, and a matching rate of plume transformation are considered the causes of this apparent steady state behaviour. Two of the selected compounds, naphthalene and 1-methylnaphthalene, continued to migrate outward despite apparent decreases in their respective input fluxes over time.

A mass balance exercise (Chapter 3) showed significant rates of plume transformation for all seven compounds selected for detailed analysis in this study. The interaction of mass flux into the plume and mass transformation within the plume caused a range of mass balance results, depending on the comparative magnitudes of these processes. These two processes exerted approximately equal influence on dibenzofuran, with the net effect that the dibenzofuran plume remained approximately static with respect to mass and position, for the last two years of monitoring. This is evidence that an organic plume can stabilize, even in the presence of an ongoing source: the first conclusive field observation of this type, that the author is aware of.

Behaviour of the $m$-xylene plume was characterized by initial mass increase followed by a decrease, as the rate of mass flux into the plume decreased to below the rate of plume transformation. Carbazole mass balance results indicated similar behaviour although the process of reversal occurred more slowly and was probably just beginning during the study period. Rates of mass flux into the plume were in excess of transformation for naphthalene and l-methylnaphthalene and, especially in the case of the former, significant additional plume expansion was expected to occur before these two processes approach a balance. 
For phenol, the rate of mass flux initially far exceeded plume transformation. The former was so large that the source was rapidly depleted with respect to phenol and a discrete slug-like plume was formed that was subjected to transformation as it migrated down gradient. Almost all of the original phenol mass in the source was transformed after $\mathbf{4 3 9}$ days. Transformation was also indicated for phenanthrene, although results were less conclusive. This compound is the most hydrophobic of those reported on herein, and the results highlighted the difficulties in evaluating natural attenuation of highly sorptive organic compounds.

In general, considerable attenuation of organic compound plumes was apparent, well in excess of that which could be expected due to hydrodynamic processes. These results indicate that there is high potential for natural attenuation to remediate plumes composed of these types of compounds, depending on the acceptable distance of plume migration. The results also highlight that attenuation rates are highly compound-specific.

Multiple lines of evidence were used to evaluate whether observed mass loss determined from the mass balance was due to biodegradation (Chapter 4). A microcosm experiment showed that indigenous aquifer microorganisms from within the creosote plume were capable of metabolizing creosote compounds aerobically. Biodegradation was most rapid for naphthalene and carbazole and nutrient addition accelerated the rate for all compounds. Accumulation of carbon dioxide in the headspace of the active microcosms and low plate counts in the controls provided additional evidence that observed compound loss was due to microbial metabolism.

Selected redox-sensitive parameters generally showed the types of changes that would be expected in association with plume biodegradation. Dissolved oxygen and $\mathrm{SO}_{4}{ }^{2-}$ decreased in groundwater within the plume while significant increases were noted for $\mathrm{Fe}^{2+}, \mathrm{Mn}^{2+}$, and methane. The behaviour of $\mathrm{NO}_{3}^{-}$was inconclusive. Further evidence that the observed mass loss was microbially-mediated was provided by accumulation of aromatic acids within the plume.

Biodegradation of plume compounds was also supported by PLFA data. Microbial biomass, evaluated on the basis of PLFA concentration, was greater within the plume, presumably due to plume-supported microbial growth. Furthermore, PLFA composition indicated that the rate of microbial turnover was higher in the plume, although it may also show that the plume was somewhat toxic to aquifer microorganisms.

Data from this study and a previous microbiological investigation at the site (Barbaro et al., 1994) indicated that results from standard plate count analysis may be strongly influenced by vertical dissolved oxygen trends, which were present in both contaminated and uncontaminated groundwater. In this scenario, plate counts may be less useful than PLFA to evaluate biomass changes in response to the introduction of organic contaminants.

Modelling dibenzofuran as a sorbing/non-decaying solute supported the suggestion that mass transformation is a strong influence on behaviour of the study plume (Chapter 5). However, modelling results indicated that the observed transformation of this compound was far in excess of 
what could be achieved by aerobic respiration proceeding to complete mineralization. Much of this excess is attributable to utilization of alternate electron acceptors, consistent with the conceptual model and supported by geochemical data. The bulk of the transformation was due to utilization of $\mathrm{O}_{2}$ and $\mathrm{SO}_{4}{ }^{2-}$ as electron acceptors. Nevertheless, the total change in electron acceptors was still not large enough to account for the observed organics loss, if transformation was assumed to proceed to complete mineralization.

Metabolite data provided qualitative evidence that a significant proportion of the organic compound biodegradation did not proceed to complete mineralization (Chapter 5). This conclusion highlights a complication in modelling biodegradation with a stoichiometric approach: transformation of a given mass of organic may require an unexpectedly small quantity of mobile electron acceptors $\left(\mathrm{O}_{2}, \mathrm{NO}_{3}{ }^{-}, \mathrm{SO}_{4}{ }^{2-}\right.$ and $\left.\mathrm{CO}_{2}\right)$ to mix with the plume, and may also account for some of the discrepancy in the electron acceptor mass balance. The effect of this process was large enough, in the study plume, to warrant incorporation of this process into the conceptual model for natural attenuation.

The distribution of dissolved oxygen was generally consistent with the conceptual model for natural attenuation of aerobically biodegradable organic compounds in groundwater (Chapter 5). However, the distribution of alternate electron acceptors within the plume core did not show a strong trend, such as those delineated at other sites. The absence of discrete redox zones may be attributable to fluctuations in groundwater flow direction which may cause instability between zones. Another possible explanation is that rate of alternate electron acceptor utilization was not sufficient to establish discrete redox zones. 


\section{CHAPTER 7 \\ RECOMMENDATIONS}

Following are some general recommendations for additional research related to this work:

1. The persistence of organic metabolites originating from several of the aromatic compounds observed in this investigation should be evaluated under various redox conditions. The purpose of this work would be to evaluate the importance of metabolites generation on a mass balance of electron acceptors versus parent compound disappearance. The study would be primarily based on laboratory experimentation and could be accompanied by plume sampling. Results could have implications for plume biodegradation and modelling.

2. Research should be conducted to develop field methods for determining in situ rates of biodegradation at various discrete plume locations. This type of information may contribute towards an improved understanding of the interaction between dynamic plume processes and plume biodegradation. It may also contribute towards improved prediction of natural attenuation and development of semi-passive methods for plume remediation.

3. Investigation of small-scale changes in microbial ecology in response to the introduction of aerobically biodegradable organic compounds should be undertaken. The purpose of this work would be to evaluate changes on microbial communities in response to new carbon sources and redox conditions, and it would involve both laboratory and field components.

4. Detailed geochemical modelling of the data set used herein should be undertaken to improve the understanding of electron acceptor utilization and distribution. 


\section{REFERENCES}

Adrian, N.R. and J.M. Suflita. 1994. Anaerobic biodegradation of halogenated and nonhalogenated N-, S-, and O-heterocyclic compounds in aquifer slurries. Environmental Toxicology and Chemistry, 13:1551-1557.

Alexander, M. 1985. Biodegradation of organic chemicals. Environmental Science and Technology, 18:106-111.

Atlas, R.M. 1981. Microbial degradation of petroleum hydrocarbons: an environmental perspective. Microbiology Reviews, 45:180-209.

Atlas, R.M. 1988. Biodegradation of Hydrocarbons in the Environment. Proceedings of a Conference on Reducing Risks from Environmental Chemicals through Biotechnology, July 19-23 in Seattle, Washington, pp. 211-223.

Baedecker, M.J., I.M. Cozzarelli, R.P. Eganhouse, D.I. Siegel and P.C. Bennett. 1993. Crude oil in a shallow sand and gravel aquifer - III. Biogeochemical reactions and mass balance modeling in anoxic groundwater. Applied Geochemistry, 8:569-586.

Baedecker, M.J. and W. Back. 1979a. Modern marine sediments as a natural analog to the chemically stressed environment of a landfill. Journal of Hydrology, 43:393-414.

Baedecker, M.J. and W. Back. 1979b. Hydrogeological processes and chemical reactions at a landfill. Ground Water, 17:429-437.

Balkwill, D.L., F.R. Leach, J.T. Wilson, J.F. McNabb and D.C. White. 1988. Equivalence of microbial biomass measures based on membrane lipid and cell wall components, adenosine triphosphate and direct counts in subsurface aquifer sediments. Microbial Ecology, 16:73-84.

Barbaro, J.R., J.F. Barker, L.A. Lemon and C.I. Mayfield. 1992. Biotransformation of BTEX under anaerobic, dentrifying conditions: Field and laboratory observations. Journal of Contaminant Hydrology, 11:245-272.

Barbaro, S.E., H.J. Albrechtsen, B.K. Jensen, C.I. Mayfield and J.F. Barker. 1994. Relationship between aquifer properties and microbial populations in the Borden aquifer. Geomicrobiology Journal, 12:203-219.

Barcelona, M.J., J. Lu and D.M. Tomczak. 1995. Organic acid derivatization techniques applied to petroleum hydrocarbon transformations in subsurface environments. Ground Water Monitoring and Remediation, Spring: 114-124. 
Barker, J.F., J.S. Tessman, P.E. Plotz and M. Reinhard. 1986. The organic geochemistry of a sanitary landfill leachate plume. Journal of Contaminant Hydrology, 1:171-189.

Barker, J.F., G.C. Patrick and D. Major. 1987. Natural attenuation of aromatic hydrocarbons in a shallow sand aquifer. Ground Water Monitoring Review, Winter: 64-71.

Bear, J. 1972. Dynamics of fluids in porous media. American Elsevier, New York.

Bennet, J.L., D.M. Updegraff, W.E. Pereira and C. Rostad. 1986. Microbial transformation of quinoline in soil at a hazardous-waste site near Pensacola, Florida. In: Movement and fate of creosote waste in ground water, Pensacola, Florida: U.S. Geological Survey Toxic WasteGround-Water Contamination Program, H.C. Mattraw and B.J. Franks eds. USGS Water-Supply Paper 2285, pp. 27-31.

Bennett, P.C., D.E. Siegel, M.J. Baedecker and M.F. Hult. 1993. Crude oil in a shallow sand and gravel aquifer - I. Hydrogeology and inorganic geochemistry. Applied Geochemistry, 8:529-549.

Berry-Spark, K.L., J.F. Barker, K.T. MacQuarrie, D. Major, C.I. Mayfield and E.A. Sudicky. 1988. The behaviour of soluble petroleum product-derived hydrocarbons in groundwater. Petroleum Association for the Conservation of the Canadian Environment. PACE Phase III Report No. 85-3.

Bjerg, P.L., K. Rugge, J.K. Pedersen and T. Christensen. 1995. Distribution of redox-sensitive groundwater quality parameters downgradient of a landfill (Grindsted, Denmark). Environmental Science and Technology, 29:1387-1394.

Bollag, J.M. and J.P. Kaiser. 1991. The transformation of heterocyclic aromatic compounds and their derivatives under anaerobic conditions. Critical Reviews in Environmental Control, 21(3,4):297-329.

Borden, R.C. and P.B. Bedient. 1986. Transport of dissolved hydrocarbons influenced by oxygenlimited biodegradation 1. Theoretical development. Water Resources Research, 22:1973-1982.

Brusseau, M.L. and P.S.C. Rao. 1989. Sorption nonideality during organic contaminant transport in porous media. CRC Critical Reviews in Environmental Control, 19:33-98.

Champ, D.R., J. Gulens and R.E. Jackson. 1979. Oxidation-reduction sequences in ground water flow systems. Canadian Journal of Earth Sciences, 16:12-23.

Chiang, C.Y., J.P. Salanitro, E.Y. Chai, J.D. Colthart and C.L. Klein. 1989. Aerobic biodegradation of benzene, toluene and xylene in a sandy aquifer - data analysis and computer modelling. Ground Water, 27:823-834. 
Cherry, J.A., R.W. Gillham, E.G. Anderson and P.E. Johnson. 1983. Migration of contaminants in groundwater at a landfill: A case study. 2. Groundwater monitoring devices. Journal of Hydrology, 63:31-49.

Cline, P.V., J.J. Delfino and P.S.C. Rao. 1991. Partitioning of aromatic constituents into water from gasoline and other complex solvent mixtures. Environmental Science and Technology, 25:914-920.

Cozzarelli, I.M., M.J. Baedecker, R.P. Eganhouse and DF. Goerlitz. 1994. The geochemical evolution of low-molecular-weight organic acids derived from the degradation of petroleum contaminants in groundwater. Geochimica et Cosmochimica Acta., 58:863-877.

Cozzarelli, I.M., J.S. Herman and M.J. Baedecker. 1995. Fate of microbial metabolites of hydrocarbons in a coastal plain aquifer: the role of electron acceptors. Environmental Science and Technology, 29:458-469.

Curtis, G.P., P.V. Roberts and M. Reinhard. 1986. A natural gradient experiment on solute transport in a sand aquifer. 4. Sorption of organic solutes and its influence on mobility. Water Resources Research, 22:2059-2067.

Devlin J.F., 1994. Enhanced in situ biodegradation of carbon tetrachloride and trichloroethene using a permeable wall injection system. Ph. D. Thesis, University of Waterloo, Waterloo, Ontario.

Dobbs, F.C. and R.H. Findlay. 1993. Analysis of microbial lipids to determine biomass and detect the response of sedimentary microorganisms to disturbance. In: Handbook of methods in aquatic microbial ecology, Lewis Publishers, pp. 347-358.

Edwards, E.A., L.E. Wills, M. Reinhard and D. Grbic-Galic. 1992. Anaerobic degradation of toluene and xylene by aquifer microorganisms under sulfate-reducing conditions. Applied and Environmental Microbiology, 58:794-800.

Edwards, E.A. and D. Grbic-Galic. 1994. Anaerobic degradation of toluene and 0 -xylene by a methanogenic consortium. Applied and Environmental Microbiology, 60:313-322.

Ehrlich, G.G., D.F. Goerlitz, E.M. Godsy and M.F. Hult. 1982. Degradation of phenolic contaminants in ground water by anaerobic bacteria: St.Louis Park, Minnesota. Ground Water, 20:703-710.

Findlay, R.H. and F.C. Dobbs. 1993. Quantitative description of microbial communities using lipid analysis. In: Handbook of methods in aquatic microbial ecology, Lewis Publishers, pp. 271284. 
Forsey, S. In progress. In situ chemical oxidation of creosote residuals. Ph. D. Thesis, University of Waterlo, Waterloo, Ontario.

Fowler, M.G., P.W. Brooks, M. Northcott, M.W.G. King, J.F. Barker and L.R. Snowdon. 1994. Preliminary results from a field experiment investigating the fate of some creosote components in a natural aquifer. Organic Geochemistry, 22:641-649.

Freyberg, D.L. 1986. A natural gradient experiment in solute transport in a sand aquifer 2. Spatial moments and the advection and dispersion of nonreactive tracers. Water Resources Research, 22:2031-2046.

Frind, E.O., E.A. Sudicky and J.W. Molson. 1989. Three-dimensional simulation of organic transport with aerobic biodegradation. In: Proceedings of the Symposium held during the Third IAHS Scientific Assembly, Baltimore, MD, May. IAHS Publ. Dev. no. 185, 89-96.

Garabedian, S.P., D.R. LeBlanc, L.W. Gelhar and M.A. Celia. 1991. Large-scale natural gradient tracer test in sand and gravel, Cape Cod, Massachusetts, 2, Analysis of tracer moments for a nonreactive tracer. Water Resources Research, 27(5):911-924.

Ghiorse, W.C. and D.L. Balkwill: Enumeration and morphological characterization of bacteria indigenous to subsurface environments. 1983. Developments In Industrial Microbiology, 24:213239.

Godsy, E.M., D.F. Goerlitz and D. Grbic-Galic. 1992. Methanogenic biodegradation of creosote contaminants in natural and simulated groundwater ecosystems. Ground Water, 30:232-242.

Goerlitz, D.F., D.E. Troutman, E.M. Godsy and B.J. Franks. 1985. Migration of wood-preserving chemicals in contaminated groundwater in a sand aquifer at Pensacola, Florida. Environmental Science and Technology, 19:955-961.

Grbic-Galic, D. 1989. Microbial degradation of homocyclic and heterocyclic aromatic hydrocarbons under anaerobic conditions. Developments In Industrial Microbiology, 30:237-253.

Grbic-Galic, D. 1990a. Anaerobic microbial transformation of nonoxygenated aromatic and alicyclic compounds in soil, subsurface, and freshwater sediments. In: Soil Biochemistry - Volume 6, eds. J.M. Bollag and G. Stotzky, Marcel Dekker, Inc. New York and Basel. pp. 117-189.

Grbic-Galic, D. 1990b. Methanogenic transformation of aromatic hydrocarbons and phenols in groundwater aquifers. Geomicrobiology Joumal, 8:167-200.

Guckert, J.B., C.P. Antworth, P.D. Nichols and D.C. White. 1985 Phospholipid ester-linked fatty acid profiles as reproducible assays for changes in prokaryotic community structure of estuarine sediments. FEMS Microbiology Ecology, 31:147-158. 
Haag, F., M. Reinhard and P.L. McCarty. 1991. Degradation of toluene and p-xylene in anaerobic microcosms: evidence for sulfate as a terminal electron acceptor. Environmental Toxicology and Chemistry, 10:1379-1389.

Hubbard, C.E., J.F. Barker, S.F. O'Hannesin, M. VanderGriendt and R.W. Gillham. 1994. Transport and fate of dissolved methanol and methyl-tertiary-butyl-ether, and monoaromatic hydrocarbons in a shallow sand aquifer. American Petroleum Institute Publication No. 4601.

Hutchins, S.R., G.W. Sewell, D.A. Kovacs and G.A. Smith. 1991. Biodegradation of aromatic hydrocarbons by aquifer microorganisms under denitrifying conditions. Environmental Science and Technology, 25:68-76.

Karickhoff, S.W. 1984. Organic pollutant sorption in aquatic systems. Journal of Hydraulic Engineering, 110:707-735.

King, M.W.G., H. Malcolmson and J.F. Barker. 1995. Groundwater plurne development from a complex organic mixture. API and NGWA Conference - Petroleum Hydrocarbons and Organic Chemicals in Ground Water: Prevention, Detection and Restoration, November 2-4, Houston, Texas.

Klecka, G.M., J.W. Davis, D.R. Gray and S.S. Madsen. 1990. Natural bioremediation of organic contaminants in ground water: Cliffs-Dow Superfund site. Ground Water, 28:534-543.

Kuhn, E.P., J. Zeyer, P. Eicher and R.P. Schwarzenbach. 1988. Anaerobic degradation of alkylated benzenes in denitrifying laboratory aquifer columns. Applied Environmental Microbiology, 54:490-496.

Lee, L.S., M. Hagwall, J.J. Delfino and P.S.C. Rao. 1992a. Partitioning of polycyclic aromatic hydrocarbons from diesel fuel into water. Environmental Science and Technology, 26:2104-2110.

Lee, L.S., P.S.C. Rao and I. Okuda. 1992b. Equilibrium partitioning of polycyclic aromatic hydrocarbons from coal tar into water. Environmental Science and Technology, 26:2110-2115.

Lee, M.D., J.M. Thomas, R.C. Borden, P.B. Bedient, C.H. Ward and J.T. Wilson. 1988. Biorestoration of aquifers contaminated with organic compounds. CRC Critical Reviews in Environmental Control, 18:29-89.

Linderfelt, W.R. and J.L. Wilson. 1994. Field study of capture zones in a shallow sand aquifer. In: Transport and Reactive Processes in Aquifers, Dracos \& Stauffer (eds.), Balkema, Rotterdam, pp. 289-294.

Linderfelt, W.R., personal communication, October-December, 1996. 
Lovley, D.R., M.J. Baedecker, D.J. Lonergan, I.M. Cozzarelli, E.J.P. Phillips and D.I. Siegel. 1989. Oxidation of aromatic contaminants coupled to microbial iron reduction. Nature, 339:297300.

Lyngkilde, J. and T.H. Christensen. 1992a. Fate of organic contaminants in the redox zones of a landfill leachate pollution plume (Vejen, Denmark). Journal of Contaminant Hydrology, 10:291307.

Lyngkilde, J. and T.H. Christensen. 1992b. Redox zones of a landfill leachate pollution plume (Vejen, Denmark). Journal of Contaminant Hydrology, 10:273-289.

MacFarlane, D.S., J.A. Cherry, R.W. Gillham and E.A. Sudicky. 1983. Migration of contaminants in groundwater at a landfill: a case study 1 . Groundwater flow and plume delineation. Journal of Hydrology, 63:1-29.

McFarland, M.J. and R.C. Sims. 1991. Thermodynamic framework for evaluating PAH degradation in the subsurface. Ground Water, 29(6):885-896.

MacIntyre, W.G., M. Boggs, C.P. Antworth and T.B. Stauffer. 1993. Degradation kinetics of aromatic organic solutes introduced into a heterogenous aquifer. Water Resources Research, 29:4045-4051.

MacKay, D., W.Y. Shiu, A. Maijanen and S. Feenstra. 1991. Dissolution of non-aqueous phase liquids in groundwater. Journal of Contaminant Hydrology, 8:23-42.

MacKay, D., W.Y. Shiu and K.C. Ma. 1992. Illustrated Handbook of Physical-Chemical Properties and Environmental Fate for Organic Chemicals, v. 1-4. Lewis Publishers.

Mackay, D.M., D.L. Freyberg and P.V. Roberts. 1986. A natural gradient experiment on solute transport in a sand aquifer 1 . Approach and overview of plume movement. Water Resources Research, 22:2017-2029.

Mackay, D.M. and J.A. Cherry. 1989. Groundwater contamination: Pump-and-Treat remediation. Environmental Science and Technology, 23:630-636.

MacQuarrie, K.T.B., E.A. Sudicky and E.O. Frind. 1990. Simulation of biodegradable organic contaminants in groundwater 1. Numerical formulation in principal directions. Water Resources Research, 26:207-222.

Madsen, E.L. 1991. Determining in situ biodegradation. Environmental Science and Technology, 25:1663-1673. 
Madsen, E.L., J.L. Sinclair and W.C. Ghiorse. 1991. In situ biodegradation: microbiological patterns in a contaminated aquifer. Science, 252:830-833.

Major, D., C.I. Maytield and J.F. Barker. 1988. Biotransformation of benzene by denitrification in aquifer sand. Ground Water, 26:8-14.

Mattraw H.C., Jr. and B.J. Franks (eds.). 1986. Movement and Fate of Creosote Waste in Ground Water, Pensacola, Florida: U.S. Geological Survey Toxic Waste-Ground-Water Contamination Program. Water Supply Paper 2285.

Mueller, J.G., P.J. Chapman and P.H. Pritchard. 1989. Creosote-contaminated sites - their potential for bioremediation. Environmental Science and Technology, 23:1197-1201.

National Research Council. 1993. Insitu bioremediation:when does it work?. National Academy Press, Washington, D.C..

Nicholson, R.V., J.A. Cherry and E.J. Reardon. 1983. Migration of contaminants in groundwater at a landfill: A case study. Journal of Hydrology, 63:131-176.

Neilsen, J.S. and S.S. Hansen. 1992. Fate and transport of creosote compounds under natural conditions in a sand aquifer at CFB Borden, Ontario, Canada. 33-Point Project, Department of Environmental Engineering, Technical University of Denmark.

Nielsen, P.H., P.L. Bjerg, P. Nielsen, P.Smith and T.H. Christensen. 1996. In situ and laboratory determined first-order degradation constants of specific organic compounds in an aerobic aquifer. Environmental Science and Technology, 30:31-37.

Patrick, G.C.. 1986. A natural gradient tracer experiment of dissolved benzene, toluene and xylenes in a shallow sand aquifer. M.Sc. Thesis, University of Waterloo.

Priddle, M.W. and K.T.B. MacQuarrie. 1994. Dissolution of creosote in groundwater: an experimental and modelling investigation. Journal of Contaminant Hydrology, 15:27-56.

Pritchard, P.H. and A.W. Bourquin. 1983. The use of microcosms for evaluation of interactions between pollutants and microorganisms. In: Advances in Microbial Ecology, ed. K.C. Marshall. Plenum Press, New York and London, pp. 133-214.

Ringelberg, D.B., J.D. Davis, G.A. Smith, S.M. Pfiffiner, P.D. Nickels, J.M. Henson, J.T. Wilson, M. Yates, D.H. Kampbell, H.W. Read, T.T. Stocksdales and D.C. White. 1989. Validation of signature polar lipid fatty acid biomarkers for alkane-utilizing bacteria in soils and subsurface aquifer materials. FEMS Microbiology Ecology, 62:39-50. 
Rivett, M.O. 1995. Soil-Gas signatures from volatile chlorinated solvents: Borden field experiments. Ground Water, 33:84-98.

Schwarzenbach, R.P., P.M. Gschwend and D.M. Imboden. 1993. Environmental Organic Chemistry. Wiley-Imterscience, New York.

Schwarzenbach, R.P. and J. Westall. 1981. Transport of nonpolar organic compounds from surface water to groundwater. Laboratory sorption studies. Environmental Science and Technology, 15:1361-1367.

Shiu, W.Y., A.L.Y. Ng and D.M. Mackay. 1988. Preparation of aqueous solutions of sparingly soluble organic substances: ii. Multicomponent systems - hydrocarbon mixtures and petroleum products. Environmental Toxicology and Chemistry, 7:125-137.

Smith, M.R. 1990. The biodegradation of aromatic hydrocarbons by bacteria. Biodegradation, 1:191-206.

Starr, R.C., J.A. Cherry and E.S. Vales. 1991. Sealable joint sheet pile cutoff walls for preventing and remediating groundwater contamination. Ontario Ministry of the Environment Technology Transfer Conference, November 25-26, Toronto, Ontario.

Stumm, W. and J.J. Morgan. 1970. Aquatic Chemistry. Wiley Interscience, New York.

Sudicky, E.A., J.A. Cherry, and E.O. Frind. 1983. Migration of contaminants in groundwater at a landfill: A case study, 4, A natural gradient dispersion test. Journal of Hydrology, 63:81-108.

Sudicky, E.A. 1986. A natural gradient experiment on solute transport in a sand aquifer: Spatial variability of hydraulic conductivity and its role in the dispersion process. Water Resources Research, 22:2069-2082.

Therrin, J., G.B. Davis and C. Barber. 1995. A ground-water tracer test with deuterated compounds for monitoring in situ biodegradation and retardation of aromatic hydrocarbons. Ground Water 33:469-475.

Tunlid, A. and D.C. White. 1992. Biochemical analysis of biomass, community structure, nutritional status and metabolic activity of the microbial communities in soil. In: Soil Biochemistry, J.M. Bollag and G. Stotzky, eds., vol. 7. Marcel Dekker, Inc., pp. 229-262.

van der Kamp, G., L.D. Luba, J.A. Cherry and H. Maathuis. 1994. Field study of a long and very narrow contaminant plume. Ground Water, 32:1008-1016.

White, D.C., W.M. Davis, S. Nickels, J.D. King and R.J. Bobbie. 1979. Determination of the sedimentary microbial biomass by extractable lipid phosphate. Oecologia, 40:51-62. 
Wilson, J.T., J.F. McNabb, D.L. Balkwill and W.C. Ghiorse. 1983. Enumeration and characterization of bacteria indigenous to a shallow water-table aquifer. Ground Water, 21:134142.

Wilson, B.H., J.T. Wilson, D.H. Kampbell, B.E. Bledsoe and J.M. Armstrong. 1990.

Biotransformation of monoaromatic and chlorinated hydrocarbons at an aviation gasoline spill. Geomicrobiology Journal, 8:225-240.

Zapico, M.M., S. Vales and J.A. Cherry. 1987. A wireline piston core barrel for sampling cohesionless sand and gravel below the water table. Ground Water Monitoring Review, Summer:74-82. 


\section{APPENDIX 1 \\ MULTI-LEVEL SAMPLER SPECIFICATIONS}

Table Al.1 Specifation of multi-level samplers added to the monitoring network through the current study.

\begin{tabular}{lrrrrr}
\hline Sampler & $\begin{array}{c}x \\
\text { coord. }\end{array}$ & $\begin{array}{c}y \\
\text { coord. }\end{array}$ & $\begin{array}{c}\text { Depth of first } \\
\text { sampling level } \\
\text { (m below grd) }\end{array}$ & $\begin{array}{c}\text { No. of } \\
\text { levels }\end{array}$ & $\begin{array}{c}\text { Vertical } \\
\text { spacing (m) }\end{array}$ \\
\hline $6-\mathrm{N} 5$ & 8.00 & -7.50 & 2.00 & 14 & 0.2 \\
$6-\mathrm{N} 5$ & 8.00 & -6.00 & 2.00 & 14 & 0.2 \\
9B-N4 & 16.75 & -7.00 & 1.80 & 14 & 0.2 \\
9B-N3 & 16.75 & -5.00 & 1.80 & 14 & 0.2 \\
$13-\mathrm{N} 4$ & 22.50 & -10.50 & 1.80 & 14 & 0.2 \\
$13-\mathrm{N} 3$ & 22.50 & -7.50 & 1.80 & 14 & 0.2 \\
$17-\mathrm{N} 3$ & 29.00 & -10.50 & 1.80 & 14 & 0.2 \\
$17-\mathrm{N} 2$ & 29.00 & -7.50 & 1.80 & 14 & 0.2 \\
$21-\mathrm{N} 3$ & 37.00 & -10.00 & 1.80 & 14 & 0.2 \\
$21-\mathrm{N} 2$ & 37.00 & -6.00 & 1.80 & 14 & 0.2 \\
$26-\mathrm{N} 2$ & 47.00 & -9.00 & 1.80 & 14 & 0.2 \\
$26-\mathrm{N} 1$ & 47.00 & -5.00 & 1.80 & 14 & 0.2 \\
\hline
\end{tabular}

Note 1: $x$-y convention is reverse of Hubbard et al. (1994)

Note 2: Hubbard (pers. comm.) stated that the depths of the shallow sampling levels are incorrectly shown in Hubbard et al. (1994) and should be $2.8 \mathrm{~m}$ rather than $1.8 \mathrm{~m}$ for the following samplers:

* 24-4

* $25-1,2,3,4,5$

* 26-0, $1,2,3,4,5$

* $27-1,2,3,4,5$

* $28-1,2,3,4,5$

* 29-1, 4, 5

* $30-1,2,3,4,5$

* 31-1, 2, 3, 4, 5

* 32-1, 2, 3, 4, 5 


\section{APPENDIX 2 \\ SYNOPTIC SAMPLING DATA}

Data on dissolved creosote concentrations from the six synoptic sampling events are provided on the computer diskette in the envelope attached to versions of this thesis on file at the University of Waterloo. Data are in field-formatted ASCII and reside in six files (filename.txt), named for the time (days) of each sampling event $(55,278,439,626,1008,1357)$. Data for the first two events were originally reported in Malcolmson (1992) and Nielsen and Hansen (1992), respectively.

A field protocol for the last event (1357 days) is attached.

Data from source cores (sand) collected in conjunction with the 626 day synoptic sampling event are provided in Table 1. Data for cores collected at 55 and 278 days were provided by Malcolmson (1992) and Nielsen and Hansen (1992), respectively. 


\section{FATE AND TRANSPORT OF CREOSOTE IN GROUNDWATER PhD Research Project - Mark King \\ Collaction of Samples for Day 1357 Snapshot \\ Written: May 1995}

\section{General}

A set of groundwater samples will be collected from approximately every second row of bundle piezometers at the emplaced creosote site. A list of piezometers to be sampled is attached as Table 1. A sample will be collected from all levels (typically 14) of each piezometer in the list. The collection period is expected to last five days. The emplaced source (ie., soil with sorbed and residual creosote) will not be cored in conjunction with this snapshot.

\section{Sampling Methods}

Samples will be collected with the manifold sampling system that has been developed in past studies done at this site. At least $50 \mathrm{~mL}$ will be purged from each sampling tube prior to sample collection. Samples will be collected in $18 \mathrm{~mL}$ gless bolles which will be sealed with crimp-top lids immediately after sampling. Samples will be stored in refijgeratod containers and kept imverted until analyzed.

The field team will consist of three to four people working with two sampling manifolds. Two people will work with the sampling manifolds and the remaining person will prepere bundle piezometers and sample bottles in advance of the samplers. The latter will do the following:

- determine which tube represents which level at each location

- replace misaing lovel tago

- replace the tubing beck in the ceaing aftor the samples are collected

- re-mark woll casings

- tape and label sample bottes

- miscellaneous running around.

The following QAQC procedures will be used for the creceste sampling:

A) Field blanks - approximately 10 of these will be collected throughout the creosote sampling period. Samples will be then with the manifold sampling system using a procedure identical to that used for piezometer sampling, ie., $50 \mathrm{ml}$ purge, etc.. They will be drawn from a bottle of deionized water that will be present throughout the sampling period. These samples will not be identified to the analytical laboratory.

B) Travel blanks - will be collected every tine a fiald blank is collected. They will be poured directly from the deionized water boltte, is., without uaing the sampling manifold. These samples will also not be identified to the analytical laboratory.

C) Field duplicates - approximately 10 of these will be collected over the sampling poriod.

D) Holding Time" samples - the purpose of these is to evaluate the eflect of excesaive holding times on sample inteority. To compose them, a 1-L semple will be collected from near the source (NS-) at 3 00-7, and further from the source (FS-) at 11-0-6. Each 1-L sample will be apportioned to $4818 \mathrm{~mL}$ sample bottles in the field labelled NS-1 through 8 and FS-1 through 8 (in triplicate). Samples 1 through 4 (total of 24 semples including tiplicates and both NS- and FS-) will be extracted, in sequence, approcimately every fouth day over the entire extraction period. They will all be analyzed on the same day, as earty as poseible in the analytical period. A record of the date of extraction and analycis will be kept. Samples 5 through 8 (NS- and FS-) will all be extracted at the same time, as 
early as possible in the extraction period (probably May 16). They will be stored with the normal snapshot extracts and will be analyzed in sequence, every third week over the entire analysis period. with a record kept of the date of analysis.

E) Method blanks - will be composed during laboratory analysis. They will be drawn from the deionized water used to rinse the gless syinge between subsampling of the samples. One method blank will be composed for each bundle piezometer (generally one every fourteen samples), as per the standard analytical protocol developed for creosote analyais

F) Spiked standards - also as per the standard protocol, spiked standards will be composed during analycis and will be identical to method blanks except that a mesaured amount of chemical standards will be added to each sample. One spike will be composed for each bundle piezometor.

\section{Sorme Problems to Avoid}

Experience in previous snapahots indicates that there is great potential for errors during collection of such a large number of samples. Some pitfalls to be aware of:

Improperty placed septa (silicon side towards the water) will result in erroneously low analytical data. Punishment for commitiing an error of this type will be switt and cruel.

- Labels may be missing on some of the bundle piezometers tubes. If there is doubt with regard to assignment of levels to tubing, it should be noted on the monitoring sheet.

Lab and field QAOC samples are extremely important and should be composed according to the frequency stated earlier.

\section{Schedule}

The following schedule is projected for field and laboratory work associated with the snapshot sampling program:

- Collection May 15 - May $19 \quad$ John, Jim, Mark, Kin on first day

- Extraction May $16-$ May $30 \quad \mathrm{Km}$, Kothorine, Michelle, Steve

- Analysis May 31 - Sept $1 \mathrm{Km}$

Some final notes re the woek in the field:

- work will proceed rain or thine, so bring some rein gear and warm clothes

- initial accommodation will be at one of the lumurious, three star motals located on the famous Wellwwy of the Stars" in beectiful downtown Angus. Later in the woek we may move to the Waterioo trailer park at Borden if the opportunity arises, so bring a sleeping bed

- you will be reimbursed for your food expenses, so bring some cash

- complimentary bug sproy and sun-block will be provided

- we will travel to the stite together earty Mondoy morning in an Earth Sci. wehiche 


\section{List of Materials}

$-18 \mathrm{ml}$ hypovials (as many as available up to 2000)

- teflon septa (2000)

- metal caps (2000)

- sample tape (15 rolls)

- sample pens (3)

- latex gloves (one box large, one box medium)

- waste bottles (total capacity of 200 litres)

- organic free water for blanks (10 litres)

- magic marker (for marking casing)

- masking tape

- sandwich bags (for samples, 200)

- 1-L brown amber sample bottles (6)

\section{List of Equipmant}

- sampling manifolds (2)

- battery (2, including Kim's)

- ice packs (as many as possible)

- coolers (7)

- crimper (a good one!)

- peristaltic pumps (Kims + one DC, one AC)

- well stocked tool kit

- 200 foot extension cord

- suction head (for taking single samples from stainiess bundles) 


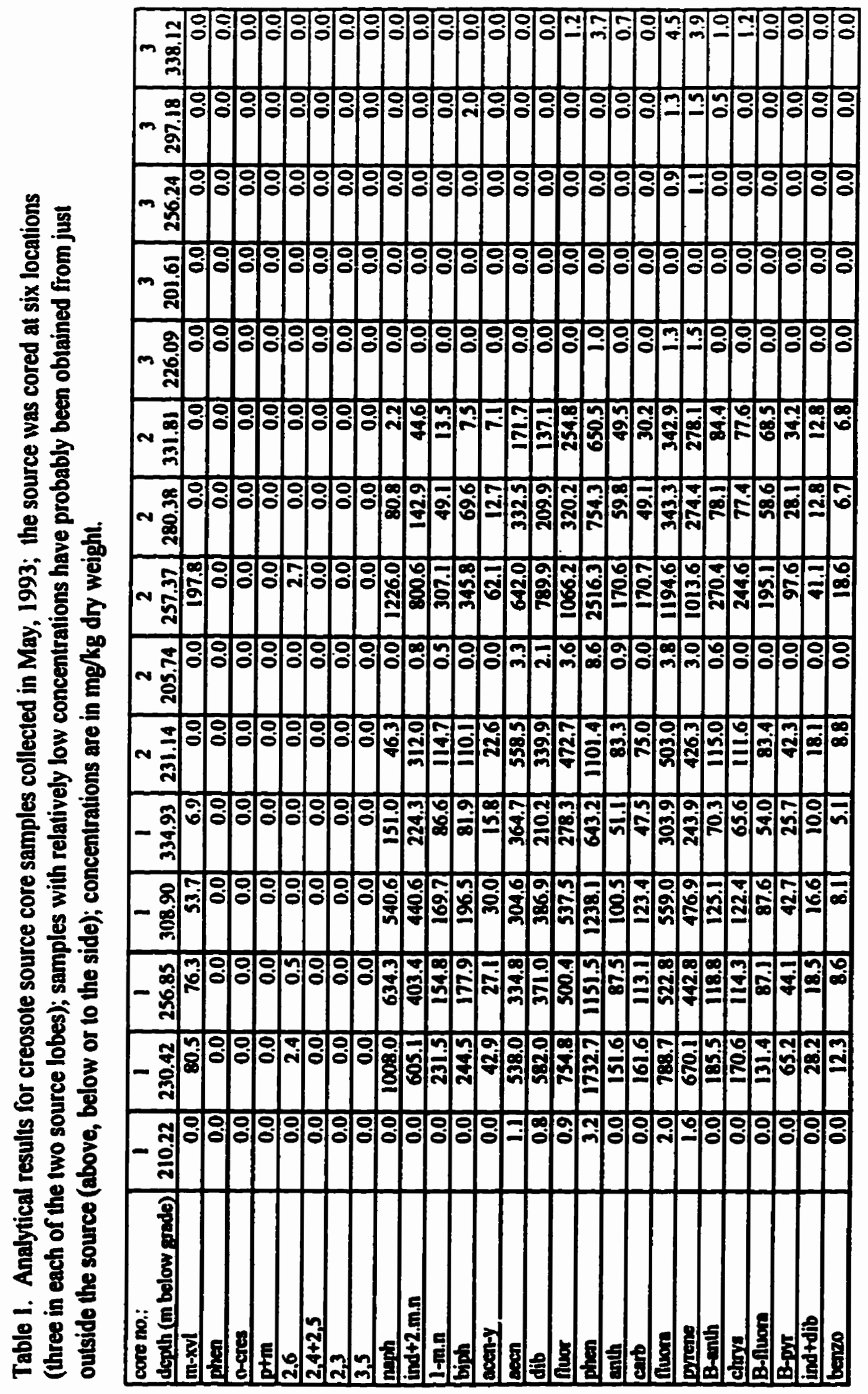




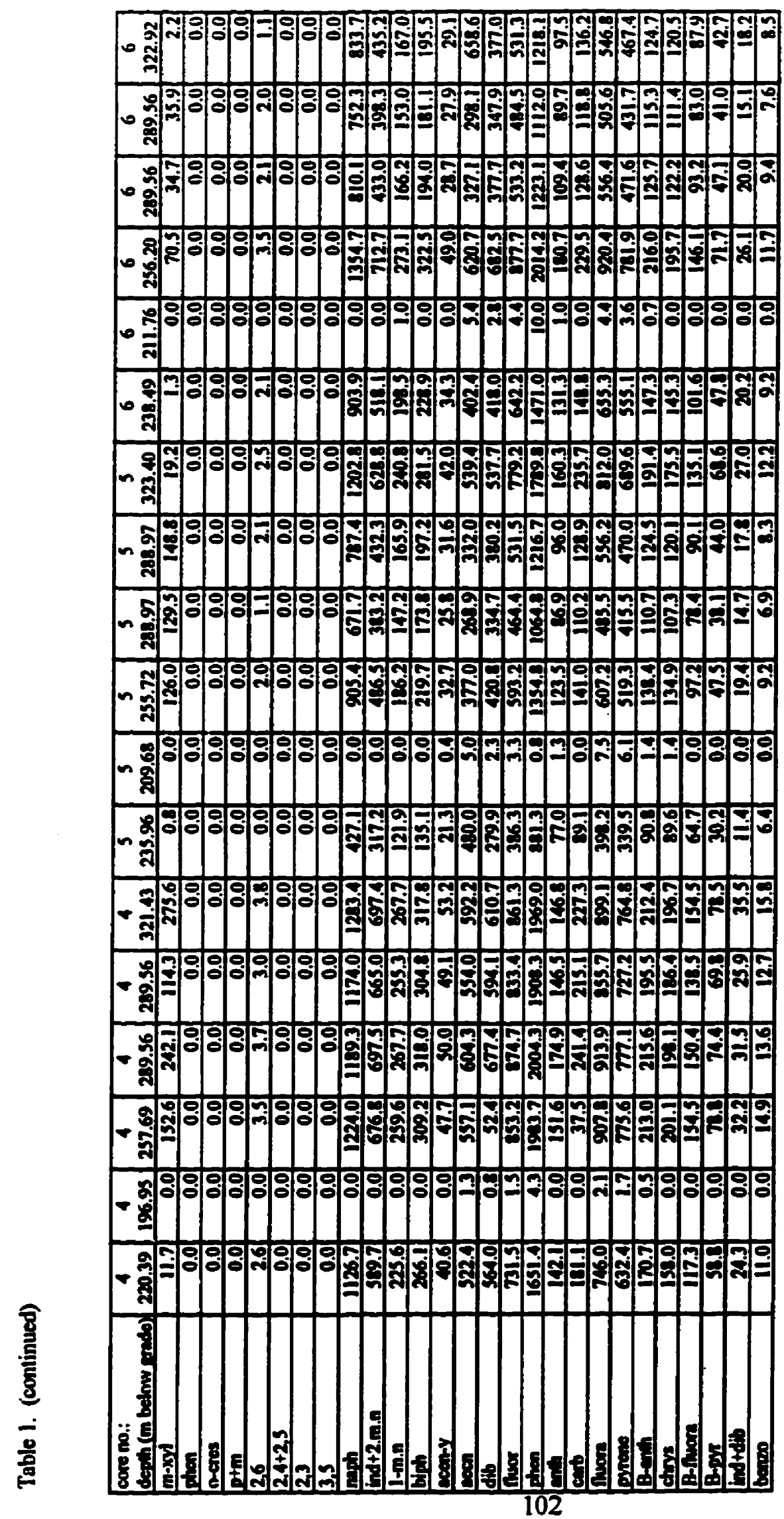




\section{APPENDIX 3 \\ TIME SERIES SAMPLING DATA}

Times series data on dissolved creosote concentrations are provided on the computer diskette in the envelope attached to versions of this thesis on file at the University of Waterloo. Data are in field-formatted ASCI in the file "timeser.txt". Time series data for the period up to 347 days after source emplacement were originally reported by Nielsen and Hansen (1992). 


\author{
APPENDIX 4 \\ Creosote Analytical Protocol: Original Method and Modifications \\ by \\ Kim Hamilton and Mark King
}

\title{
1.0 INTRODUCTION
}

An analytical protocol has been developed for the quantification of selected creosote compounds in water, sand and pure creosote. The method was developed during the spring and summer of 1991 and was first used for analysis of actual samples in the summer of 1991. Since it was developed the method has undergone several modifications to improve accuracy and sensitivity. All samples quantified for creosote compounds before spring 1993 were analyzed according to the original protocol while more recent anahysis has been performed according to the modified methodology. In the following, both methods are described in detail. Differences between the two methods are indicated in the section describing the modifications.

\subsection{ORIGINAL METHOD}

\section{Overview}

This method was developed to anabyze for the following creosote components: $m$-xylene, phenol, p-cresol, 2,6-dimethyl phenol, naphthalene, anthracene, phenanthrene, dibenzofuran, carbazole, pyrene, indole, 2-methyl naphthalene and 1-methyl naphthalene; however, it mey be validated for other compounds as required.

\section{Chemicals and Solvents}

M-xylene, 99\% purity, BDH

Phenol, $99+\%$ purity, Aldrich

P-cresol, 99\% purity, Sigma

2,6-dimethyl phenol, $99 \%$ purity, Sigma

Naphthalene, 99+\% purity, Sigma

Phenanthrene, $98+\%$ purity, Sigma

Anthracene, $99+\%$ purity, Sigma

Dibenzofuran, $99+\%$ purity, Aldrich

Carbazole, 95\% purity, Sigma

Pyrene, $99+\%$ purity, Aldrich

Indole, 99+\% purity, Aldrich

2-methylnaphthalene, $98 \%$ purity, Addrich

1-methylnaphthalene, $88 \%$ purity, Addrich

Dichloromethane

Methanol

Sodium Chloride

Hydrochloric Acid 


\section{Preparation of Stock Standards}

Two stocks are composed because it has been reported that some phenolic compounds degrade over time in the presence of some of the more hydrophobic compounds.

\begin{tabular}{|c|c|c|c|}
\hline Stock A: & $\begin{array}{l}\text { m-xylene } \\
\text { phenol } \\
\text { p-cresol } \\
\text { 2,6-dimethyl phenol } \\
\text { naphthalene } \\
\text { phenanthrene }\end{array}$ & $\begin{array}{l}\text { Stock B: } \\
\text { carbazole }\end{array}$ & $\begin{array}{l}\text { dibenzofuran } \\
\text { pyrene } \\
\text { indole } \\
\text { 2-methyinaphthalene } \\
\text { thalene }\end{array}$ \\
\hline
\end{tabular}

To make the stocks, accurately weigh out approximately $80 \mathrm{mg}$ of each compound (80-90 ul of the liquids) into a $100 \mathrm{ml}$ volumetric flask. Add $80 \mathrm{mi}$ MoCl2, sonicate to dissolve all compounds. Make to the mark with MeCl2. This gives a stock of about $800 \mathrm{mg} / \mathrm{l}$ of each compound. Store tightly sealed in the freezer in a brown bottle. Because p-cresol is a semi-liquid, it is easier to waigh it directly into the volumetric flask instead of using a weighing vessel. M-xylene and 1methylnaphthalene are both liquids. 2-methylnaphthalene is a moist solid and may be weighed on a paper or directly into the volumetric flask.

\section{Preparation of Calibration Standards}

Dilute $1 \mathrm{ml}$ of the above stock solutions into $100 \mathrm{ml}$ MeC2, shake well. Store standards in the freezer in a brown bottle until ready to use. Transfer approximately $1 \mathrm{ml}$ to an autosampler vial when needed. The concentrations of these standards will be $100 \mathrm{th}$ that of the stock solutions $(8 \mathrm{mg} / \mathrm{l})$.

\section{Preparation of Spikes}

Spikes are prepared to check the efficiency of compound extraction from water. Prepare a second set of stock standards of $40 \mathrm{mg}$ of each compound into $100 \mathrm{ml}$ MeOH. For $45 \mathrm{ml}$ samples add $45 \mathrm{ml}$ organic free water, $1 \mathrm{ml} 1 \mathrm{~N} \mathrm{HCl}$ and $9 \mathrm{~g} \mathrm{NaCl}$ to a $60 \mathrm{ml}$ crimp top vial, crimp and shake. Spike in 60ul of each of the above stock solutions. For $13 \mathrm{ml}$ samples, add $13 \mathrm{ml}$ organic free water, $0.5 \mathrm{ml} 1 \mathrm{~N} \mathrm{HCl}$ and $3 \mathrm{~g} \mathrm{NaCl}$ to a $20 \mathrm{ml}$ crimp top vial, crimp and shake. Spike in $20 \mathrm{ul}$ of each of the above stock solutions. Follow the extraction procedure for the appropriate sample size. Spikes are prepared at the same time as samples.

\section{Preparation of Elanks}

The purpose of blanks is to check the solvent, $\mathrm{NaCl}$ and $\mathrm{HCl}$ for contamination. They are prepared in an identical manner to the spikes except that the standard compounds are not added to the solvent. 


\section{Preparation of 800 Standard}

The $\mathbf{8 0 0}$ sample is run to help identify compounds that do not have standards and for monitoring long term performance of the Gas Chromatograph (CC). It is so named because it is composed using creosote that was collected at the Borden field site at $8 \mathrm{AM}$ on the moming that the creosote source was installed. Make a $100 u$ creosole / $100 \mathrm{ml}$ MeC12 dilution. Store in the freezer in a brown bottle until needed. Transfer approximately $1 \mathrm{ml}$ into an autosampler vial for analysis.

\section{Preparation of Groundwater Samples}

Typical sample sizes and associated quantities of amendments and solvents used in sample preparation are provided in Table 1. Depending on the size of the sample, use a syringe to transfer either 13, 45 or $100 \mathrm{ml}$ of sample to a clean, crimp top vial of appropriate size. Add $\mathrm{NaCl}$ and $1 \mathrm{~N}$ $\mathrm{HCl}$, crimp, shake by hand to dissolve salt. $\mathrm{Add} \mathrm{MeCl}_{2}$ with syringe through the septa, shake 20 min at 350-400rpm. Remove approximately $1 \mathrm{ml}$ of $\mathrm{MeCl}_{2}$ by inverting the vial and inserting a syringe, transfer to an autosmapler vial.

Table 1. Quantities of amendments and solvent used for water sample extraction

\begin{tabular}{|c|c|c|l|}
\hline \hline Sample Size & wt. NaCl & vol $1 \mathrm{~N} \mathrm{HCl}$ & vol MoCl \\
\hline & & & \\
\hline $13 \mathrm{ml}$ & $3 \mathrm{~g}$ & $0.3 \mathrm{ml}$ & $1.0 \mathrm{ml}$ \\
\hline $45 \mathrm{ml}$ & $9 \mathrm{~g}$ & $1.0 \mathrm{ml}$ & $3.0 \mathrm{ml}$ \\
\hline $100 \mathrm{ml}$ & $0 \mathrm{~g}$ & $0 \mathrm{ml}$ & $3.0 \mathrm{ml}$ \\
\hline
\end{tabular}

\section{Sample Sequence}

A sample run starts with analysis of: a blank

800 sample

Then, after every 10 samples, run a spike, Standard A, Standard B and an 800 sample. 


\section{Gas Chromatograph Information}

Helium carrier $30 \mathrm{~cm} / \mathrm{sec}$

Helium makeup gas $24 \mathrm{~m} / \mathrm{min}$

Hydrogen $30 \mathrm{ml} / \mathrm{min}$

Air $300 \mathrm{~m} / \mathrm{min}$

Injector temperature: 275 degrees C

FID temperature: 325 degrees $C$

Oven: 40 degrees $C$ for $0.5 \mathrm{~min}, 15$ degrees $C$ to 300 degrees $C$, hold $2 \mathrm{~min}$

Separation time: 19.33

Injection size: 2ul

Attenuation: $\mathbf{2}^{2}$

Quantification

A response factor (Rf) is obtained for each of the standard compounds by injecting $2 u$ of the calibration standard and the following equation:

$$
R f(m g / / a r e a)=\frac{\text { standard concentration }(m g / 1)}{\text { peak area }}
$$

The concentration of analyte in water $\left(C_{m}\right)$ is calculated using the peak area, $R f$ and the fraction of compound recovered $(R)$ through anahysis of the spike. Also, the result incorporates the sample volume (SV) and the volume of $\mathrm{MeCl}_{2}$ injected into the sample:

$$
C_{m q}(m g / l)=\frac{\text { peak area }-R f(m \circ / \text { Varea })}{(S V(m)) / M e C l 2(m))-R}
$$

Concentration data for spikes are examined for the degree of compound recovery. If recovery is between $80 \%-120 \%$, as it typically is for most compounds, a recovery correction factor is not applied to the data. However, in the case of phenol recovery is typically around $50 \%$. An R of 0.5 is therefore used for phenol to correct for the poor recovery.

\section{Method Detection Limit:}

These are summarized in Table 2.

\section{Additional Information}

Peaks were identified for some additional compounds including: acenaphthene, fluorene, methyl phenanthrene, fluoranthene, naphthacene and chrysene. However, a source of standards for these compounds was not found during development of the original protocol. Instead, concentrations were estimated using the average of the response factors for phenanthrene and anthracene. Similarty, the average of the response factors for indole, 2-methyl naphthalene and 1-methyl naphthalene was used as an approximation of the response factor for biphenyl and for subsequent quantification. 


\section{Analysis of Pure Creosote Samples}

To analyze pure creosote: dissolve a 10 ul subsample in $10 \mathrm{ml}$ dichloromethane. After agitation, transfer $1 \mathrm{ml}$ of the solution to a $2 \mathrm{ml}$ glass hypovial for injection into the GC. Concentrations in pure creosote $\left(C_{\text {croo }}\right)$ are quantified using the following:

$$
C_{\text {eno }}(u g / m l)=\text { peak area * Rf(mg/Varea) * (MeCl2(ul)/creo(ul)) }
$$

\section{Analysis of Sand (Source) Samples}

The mass of sand that has been sampled and extrected has varied between sample sets. However, the basic analytical procedure is the same. A measured mass of creosote/water wet sand is sampled directly into a glass hypovial (capacity varied between sample sets). The sample is rinsed in the original sample bottle at least three times with dichloromethane. All dichloromethane is collected and evaporated to less then $3 \mathrm{ml}$. The solvent volume is measured and $2 \mathrm{ml}$ is placed in an autosampler vial for GC injection.

In order to express compound concentrations on a dry sand weight basis, the sand sample is weighed before solvent extraction and then weighed again affer extraction to determine the fraction of the bulk soil mass due to moisture and creosote. Concentrations in sand $\left(C_{\text {ande }}\right)$ are calculated using the concentrated solvent volume (CSV) and the dry sample weight (DSW) by the following:

$$
C_{\text {mend }}(\mathrm{mg} / \mathrm{kg})=\frac{\text { peak areg } * \text { Rf }(\mathrm{mg} / \text { Nareg }) \cdot \operatorname{csV} 0}{\operatorname{DSW}(\mathrm{kg})}
$$

\subsection{METHOD MODIFICATIONS}

\section{Overviow}

The method has been modified to increase analytical capabilities and to improve performance. Modifications are summarized as follows:

1) Instead of making the stock solutions by adding pure form compounds to solvent, ampules of pre-measured compounds in solvent are now used for certain compounds. A pro-mix of 16 PAHs is used as well as pro-mixes of m-xylene, dibenzofuren and 2-methylnaphthalene.

2). Due to the pre-mixes mentioned in 1), direct analysis for severel PAHs has been added to the protocol, including: acenaphthene, fluorene, fluoranthene, chrysene, acenaphthylene, benzo(o)anthracene, benzo(b)filuoranthene, benzo(k)fluoranthene, benzo(a)pyene, indeno $(1,2,3-c, d)$ pyrene and dibenzo $(a, h)$ pyrelene. The first four of these were analyzed through the original protocol but quantification was indirect since it made use of response factors estimated from other compounds.

3) Direct analysis for biphenyl was added. Previoushy, biphenyl concentrations were estimated using response factors for other compounds. 
4) Capability was also added for analysis of 2 cresols (o-cresol and $m$-cresol) and four dimethylphenols $(2,4 \mathrm{dmp}, 2,6 \mathrm{dmp}, 2,3 \mathrm{dmp}$ and 3,5 dmp. Standards for these are composed using pure compounds and not pre-mixed ampules.

5) It was recognized that several compounds elute together and are therefore indistinguishable from each other by this protocol. Included in this group are the following pairs: $p+m$ cresol, $2,4+2,5$ dmp. indole +2 -methylnaphthalene, benzo(b)fluoranthene + benzo(k)fluoranthene, indeno(1,2,3-c,d)pyrene + dibenzo $\left(a_{2} h\right)$ anthracene. Combined concentrations are determined for these pairs.

6) Calibration standards are now injected into water (organic free) and extracted back out instead of direct analysis in solvent. The new procedure is more representative of the way that actual samples are extracted and therefore diminishes concerns regarding compound recovery.

7) As a result of 6), it is no longer necessary to correct phenol concentrations for recovery.

6) An interrial standard (2-fluorobiphenyl) was incorporated to account for possible inaccuracies in sample and/or solvent measurement.

Results evaluating analytical bias versus sample concentration are shown in Table 3. As discussed above, analysis of several parameters was subject to certain limitations, including: a) use of estimated response factors b) co-elution with other compounds and c) exclusion from the original protocol. Subsequent interpretation was primarily focussed on compounds that were not subject to these limitations, as summarized in Table 4.

\section{Preparation of Stock Standards}

\section{Stock A:}

- phenol

- $\quad$ all cresols (3)

- all dimethylphenols (5)

Weigh to five decimal places $5 \mathrm{mg}$ of each compound (10ul of the liquids) and place in a $50 \mathrm{ml}$ volumetric flask. Add $20 \mathrm{ml}$ MeOH, sonicate to dissolve all the compounds. Make to the mark with MeOH. This gives a stock of about $100 \mathrm{ug} / \mathrm{ml}$ of each compound. Store tightly sealed in the freezer in a brown bottle.

Stock B:

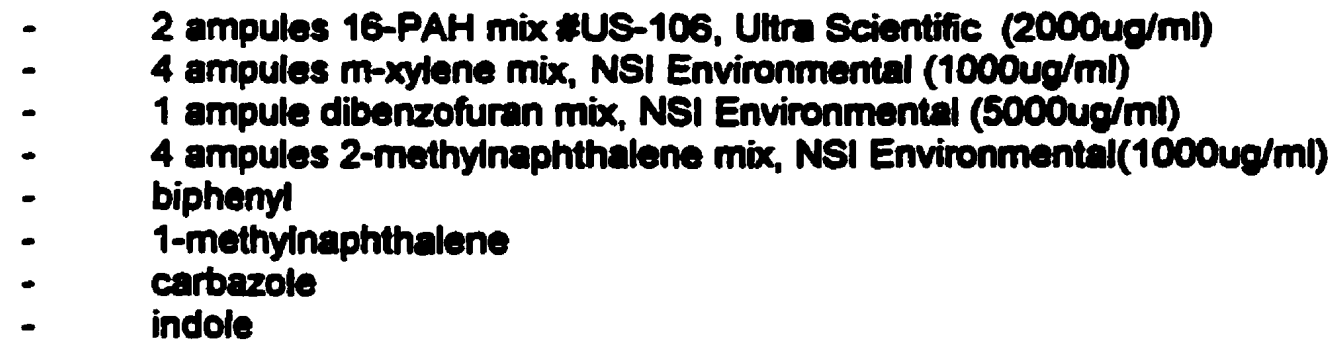

Combine in a $50 \mathrm{ml}$ volumetric flask the above mix's (rinse ampules with MeOH, add rinses) and $4 \mathrm{mg}$ (weighed to five decimal places) of each of the individual compounds (4). Add $35 \mathrm{ml}$ MeOH and 
sonicate to dissolve all compounds, make to volume with $\mathrm{MeOH}$. This gives a stock of approximately $80 \mathrm{ug} / \mathrm{ml}$. This solution is then divided into $50-1 \mathrm{ml}$ amber ampules, sealed, and stored in the freezer.

\section{Preparation of Calibration Standards}

100ul of each of the above stock solutions are spiked in water and extracted as a sample. Check standards are prepared at $1 / 10$ th the concentration.

In contrast to the original method, spikes are not required since the calibration standards are composed in water.

\section{Preparation of Blanks}

A solvent blank is composed by adding $\mathrm{MeCl}_{2}$ to an autosampler vial and is intended to verify the integrity of the solvent. A method blank is a sample composed of organic free water only. It is extracted in a manner identical to a regular sample and is intended as a check for accidental laboratory contamination.

\section{Preparation of 800 Standard}

Procedure is identical to the original protocol.

\section{Preparation of Groundwater Samples}

To the appropriate size of clean, dry crimp top vials add the listed amounts of $\mathrm{NaCl}$ and $1 \mathrm{~N} \mathrm{HCl}$ from Table 1. Transfer the sample using a 30mt glass syninge from its original vial to the vial with the salt and acid, crimp, shake by hand to dissolve the salt. Add the appropriate amount of $\mathrm{MeCl}_{2}$ (containing 10ppm of the internal standard 2-lluorobipheny), shake $15 \mathrm{~min}$ at 350-400 $\mathrm{pm}$. Remove about $1 \mathrm{ml}$ of dichloromethane by inverting the vial and withdrawing the solvent into a syringe. Transfer the solvent to an autosampler vial and analyze. The intemal standard is used to account for any sample volume or injection volume changes thet may occur.

\section{Sample Sequence}

A sample run starts with: solvent blank method blank standard check standard 800 sample

After every 10th sample: standard check standard

After every 20th sample: blank

800 standard 


\section{Gas Chromatograph information}

Identical to the original protocol with one exception:

Injection size: 3ul (previously 2ul)

\section{Quantification}

All standards and samples are normalized to the area of one standard (usually the first one run for a batch of samples) using the areas obtained from the internal standards (IS) and the following equation:

corrected peak area = peak area * is area from standard: 1

is area from sample

The response factors (RI) for each analyte are calculated using the following equation:

$$
R f=\frac{\text { standard concentration }}{\text { peak area }}
$$

For each analyte, the concentration of analyte in water $C_{m p}$ is calculated using:

$$
C_{\infty}=\text { corrected peak area } \bullet \mathbf{R f}
$$

Method Detection Limits

These are shown in Table 2.

Analysis of Pure Creosote Samples

Identical to original protocol

Analysis of Sand (Source) Samples

Identical to original protocol 
Table 2. Method Detection Limits for Creosote Compounds in Water (all concentrations are ug/)

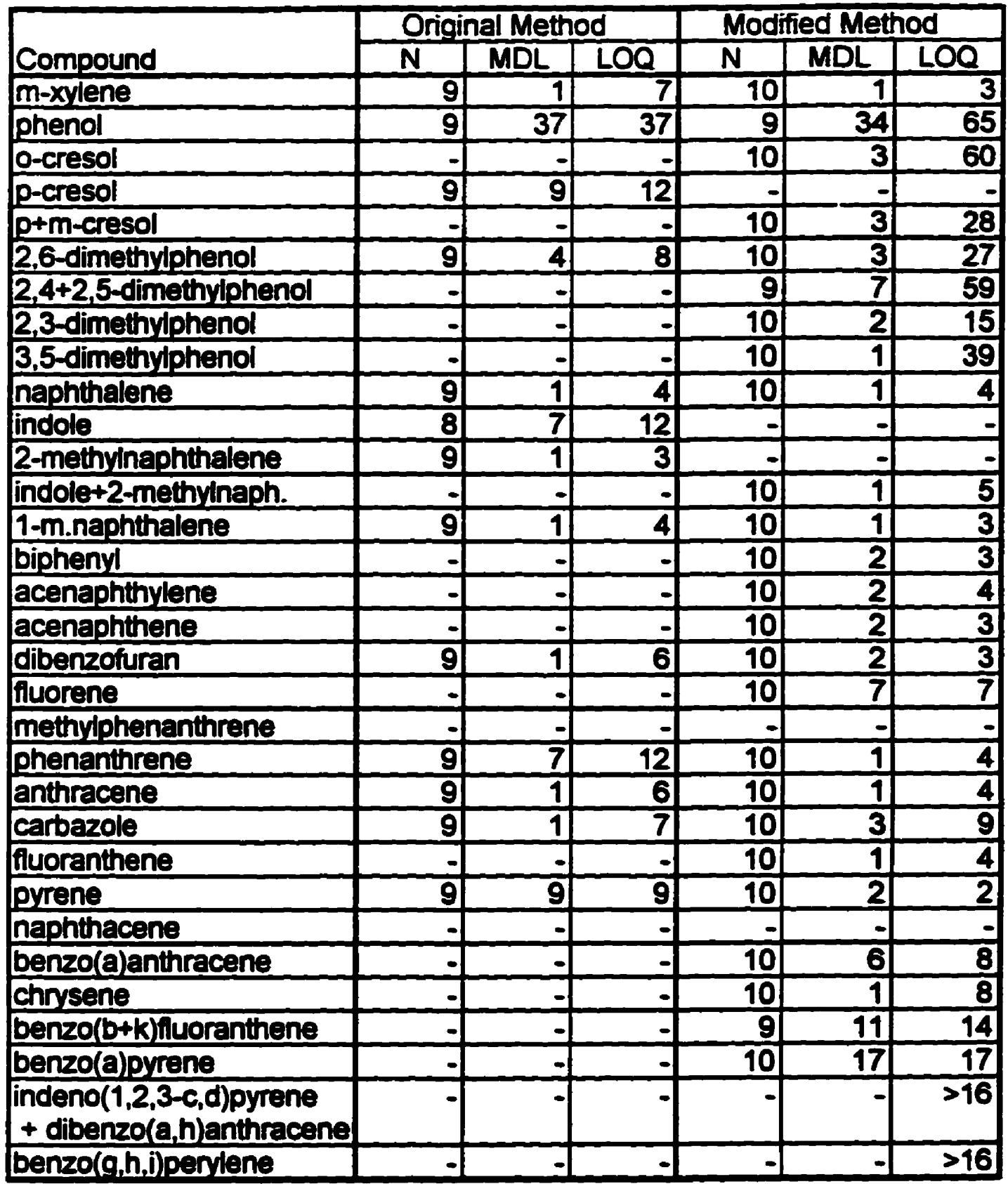

Comments:

1. $\mathbf{N}$ - number of replicates used in analysis

2. MDL - Method Detection Limit; calculated based on standard deviation

3. LOQ - Limit of Quantification, smallest quantity that can be reliably determined

4. All concentrations have been rounded to the nearest ugh 
Table 3. Evaluation of analytical bias on the basis of spiked concentration versus percent error; spiked concentrations are averages of triplicate results.

\begin{tabular}{|l|rr|rr|lr|rr|}
\hline & $\mathrm{mg} / \mathrm{l}$ & \% error & $\mathrm{mg} / \mathrm{l}$ & \% error & $\mathrm{mg} / \mathrm{l}$ & \% error & $\mathrm{mg} / \mathrm{l}$ & \% error \\
\hline m-xylene & 0.923 & -0.28 & 0.231 & -3.54 & 0.115 & -3.09 & 0.058 & -1.53 \\
phenol & 1.636 & -5.00 & 0.409 & -8.70 & 0.204 & 3.73 & 0.102 & -3.20 \\
2,6-dmp & 1.209 & 3.32 & 0.302 & -0.75 & 0.151 & -1.32 & 0.076 & 0.96 \\
naphthalene & 0.819 & 0.31 & 0.205 & 4.32 & 0.102 & -3.79 & 0.051 & -3.02 \\
1-m.naph & 0.890 & -4.20 & 0.222 & -1.93 & 0.111 & 4.29 & 0.056 & 3.76 \\
dibenzofuran & 0.979 & 1.44 & 0.245 & 2.20 & 0.122 & 1.17 & 0.061 & -3.42 \\
phenanthrene & 0.819 & 0.11 & 0.205 & -2.42 & 0.102 & -4.18 & 0.051 & -4.42 \\
anthracene & 0.819 & -1.07 & 0.205 & 1.19 & 0.102 & 2.36 & 0.051 & 3.33 \\
carbazole & 1.808 & 3.63 & 0.452 & 3.41 & 0.226 & 4.02 & 0.113 & 4.76 \\
pyrene & 0.819 & 2.36 & 0.205 & 4.24 & 0.102 & 4.67 & 0.051 & 4.14 \\
\hline
\end{tabular}


Table 4. Evaluation of data record for analytical parameters

\begin{tabular}{|c|c|c|c|c|}
\hline Compound & $\begin{array}{l}\text { Estimated } \\
\text { response } \\
\text { factors } \\
\text { used for } \\
\text { eariy data }\end{array}$ & $\begin{array}{c}\text { Compounds } \\
\text { that } \\
\text { co-elute }\end{array}$ & \begin{tabular}{|c|} 
Not \\
analyzed \\
in \\
original \\
protocol
\end{tabular} & $\begin{array}{l}\text { Recommended } \\
\text { for focus } \\
\text { of interp'n } \\
\text { and } \\
\text { discussion }\end{array}$ \\
\hline m-xylene & & & & $x$ \\
\hline phenol & & & & $\bar{x}$ \\
\hline o-cresol & & & $\bar{x}$ & \\
\hline p-cresol & & $\bar{x}$ & & \\
\hline$p+m-c r e s o l$ & & $\bar{x}$ & $\bar{x}$ & \\
\hline 2.6-dimethyiphenol & & & & $\bar{x}$ \\
\hline 2,4+2,5-dimethylphenol & & $\bar{x}$ & $\bar{x}$ & \\
\hline 2.3-dimethydphenol & & & $\bar{x}$ & \\
\hline 3.5-dimethylohenol & & & $\bar{x}$ & \\
\hline naphthalene & & & & $\bar{x}$ \\
\hline indole & & $\bar{x}$ & & \\
\hline 2-methyinaphthalene & & $\mathbf{x}$ & & \\
\hline indole+2-methyinaph. & & $\underline{x}$ & $\underline{\mathbf{X}}$ & \\
\hline 1-m.naphthalene & & & & $\mathbf{x}$ \\
\hline biphenyl & $\mathbf{x}$ & & & \\
\hline acenaphthylene & & & $\bar{x}$ & \\
\hline acenaphthene & $\bar{x}$ & & & \\
\hline dibenzofuran & & & & $\bar{x}$ \\
\hline fluorene & $\bar{x}$ & & & \\
\hline methylphenanthrene & $\bar{x}$ & & & \\
\hline phenanthrene & & & & $\bar{x}$ \\
\hline anthracene & & & & $\mathbf{x}$ \\
\hline carbazole & & & & $\underline{x}$ \\
\hline fluoranthene & $\bar{x}$ & & & \\
\hline pyrene & & & & $\bar{x}$ \\
\hline naphthacene & $\underline{x}$ & & & \\
\hline benzo(a)anthracene & & & $\underline{\mathbf{x}}$ & \\
\hline chrysene & $\mathbf{x}$ & & & \\
\hline benzo $(b+k)$ fluoranthene & & $\underline{x}$ & $x$ & \\
\hline benzo(a)pyrene & & & $\bar{x}$ & \\
\hline $\begin{array}{l}\text { indeno }(1,2,3-c, d) \text { pyrene } \\
+ \text { dibenzo(a,h)anthracene }\end{array}$ & & $\mathbf{x}$ & $\mathbf{x}$ & \\
\hline benzo(g,h,i)perylene & & & $\underline{x}$ & \\
\hline
\end{tabular}


APPENDIX 5

CHARACTERIZATION OF THE STUDY CREOSOTE

Conducted by Paul Brooks

Institute for Sedimentary and Petroleum Geology

Calgary, Alberta 
The coal tar oupplied (Wäterloo) was separated into 2 fractions; viz. hydrocarbons and resine (ooe scheme 1). Each fraction was examined by GC-MS.

A further sample of the coal tar was treated with a dilute solution of NaOH and extracted with dichloromethane (see scheme 2). The residual aqueous layer was actdified with HCl and extracted again with dichloromethane. This provided an "acid" fraction of the coal tar which was subsequently examined by GC-MS.

An allquot of the coal tar was shaken very gently in excess water (Bee schene 3 ). No exulsion was evident. An allquot of the aqueous layer was carefully taken, acldified and extracted with dichloromethane. This provided a readily water soluble frection of the coal tar. Ihis fraction was examined by GC-MS.

Prsurets

\section{A) Hydrocarbons}

Flgure 1 abowe the Total Ion Current (TIC) of the hydrocarbon fraction from coal tar. The trace has been annotated with compound Identities beeed on data base 1ibrary searching and on the basie of retention times compared to the literature. Naturally, tho major components are polynuclear aromatice (PNA's) with a minor contribution from oulphur containing compounds ouch as benzothiophene and dibenzothiophene. Table 1 liots the major components present in the fraction.

$\begin{array}{lc}\text { Compound } & \text { conce } \\ \text { Indene } & + \\ \text { naphthalene } & ++++ \\ \text { 2-methyl naphthalene } & ++ \\ \text { l-nethyl naphthalene } & ++ \\ \text { acenaphthalene } & + \\ \text { blphenyl } & + \\ \text { dinethylnaphthalenes } & + \\ \text { dibenzofuran } & ++ \\ \text { Eluorene } & +++ \\ \text { phenanthrene } & +++ \\ \text { anthracene } & ++ \\ \text { nethyl phenanthrenes } & ++ \\ \text { cyclopenta phenanth. } & ++ \\ \text { fluoranthene } & +++\end{array}$

$\begin{array}{lc}\text { Compound } & \text { cone } \\ \text { pyrene } & ++ \\ \text { benzo (a) fluorene } & + \\ \text { benzophenanth. } & ++ \\ \text { chryeene } & ++ \\ \text { benzofluoranthene }(\mathrm{b})+ \\ \text { benzofluoranthene }(\mathrm{B})+ \\ \text { benzo( } \mathrm{+}) \text { pyrene } & + \\ \text { benzo(A)pyrene } & + \\ \text { perylene } & + \\ \text { benzperylenes } & +\end{array}$




\section{B) Resin Eraction}

Figure 2 shows the TIC of the "resin" fraction of the coal tar. The trace is extremely complex. The trace has been annotated with tentative identifications of the major compones:s based on library search of the full mass epectra. Thus, the fraction appears to be dominated by phenols and substituted phenols and by nitrogen containing PNA's. Table 2 sumarizes the major components present.

\section{Compound}

phenol

methyl phenola

dimethyl phenols

ethyl, methyl phenole

isoquinoline

benzene acetonitrile

methylquinolines

dimethylquinolines

methyl indole

carbazole

benzoguinolines

nitroeocarbazole?

anthracene carbonitrile

fluorene carbonitrile

fluranthereamine

benzocarbazoles

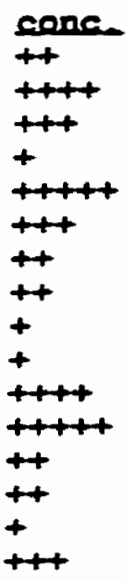

c) Acid fraction

Fibure 3 shows the IIC for the "ecld" fraction from the coal tar. As previously, the trace 18 annotated with the Identifications of the major components besed on l1brary search and GC retention time. The major components appear to be phenol, oubetituted phenola and oxygen containing compounde. Table 3 lists the major components Identified.

compound

phenol

2-nethyl phenol

4-methyl phenol

dimethyl phenols

ethyl, methyl phenol

naphthalenole or

phenyl furans

methyl naphthalenols.

biphenyl-ols

dibenzofuranola

phenanthrenole

benzonapthofurane

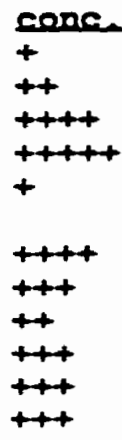




\section{D) Water soluble components}

After gently shaking an aljquot of coal tar with water, the aqueous layer was acidified and extracted with dichloronethane. The reaulting mixture was examined by ce-ms. The TIC obtained is skown in fleure 4 annotated with compound identifications based on library search and ge retention time. The major components are phenol, methyl phenols and dimethyl phenols. The major component eluting early in the chromatogram is posaibly benzonitrile, based on ribraxy search. The unly PNA detected, which was only present in minor concentrations, was naphthalene. 


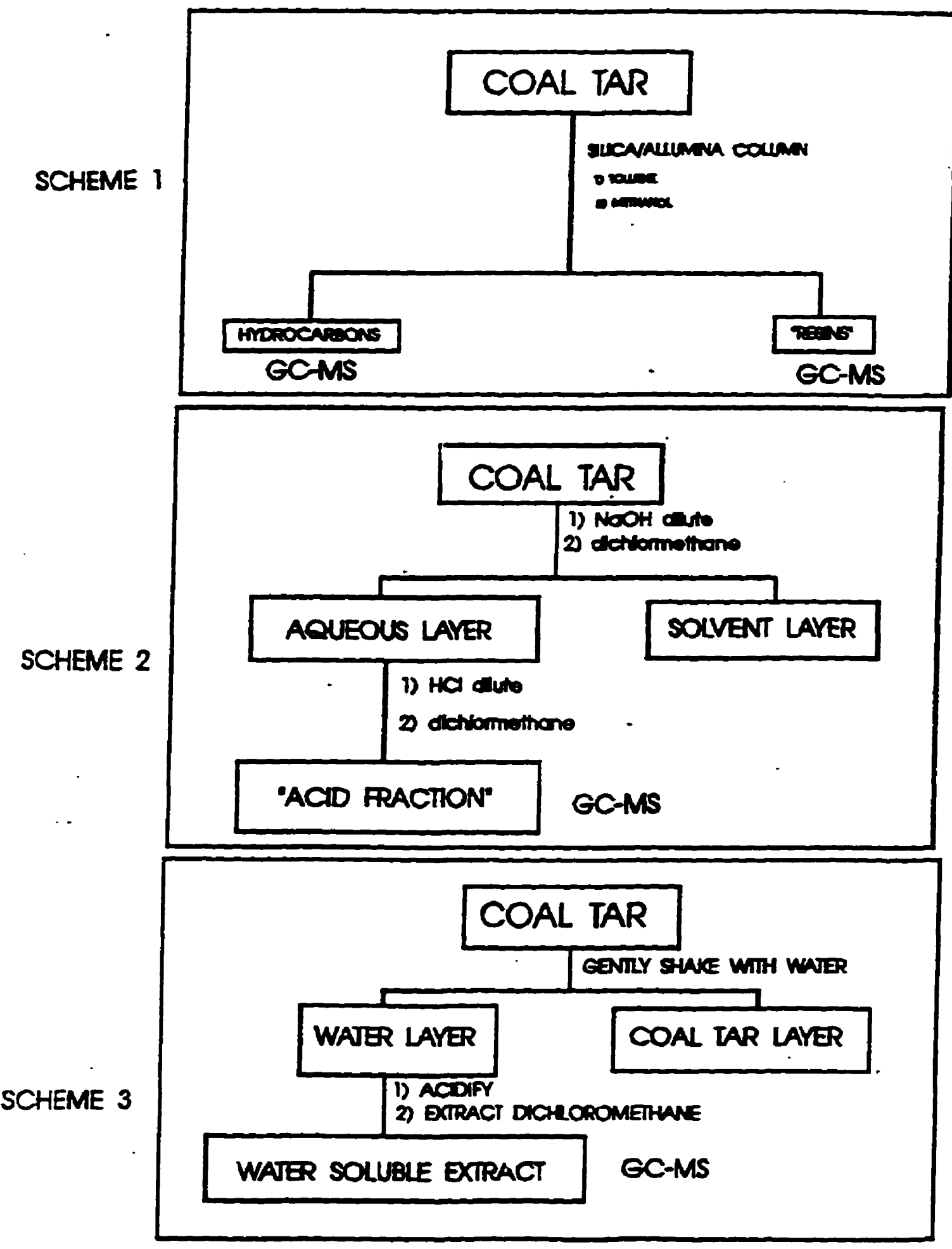




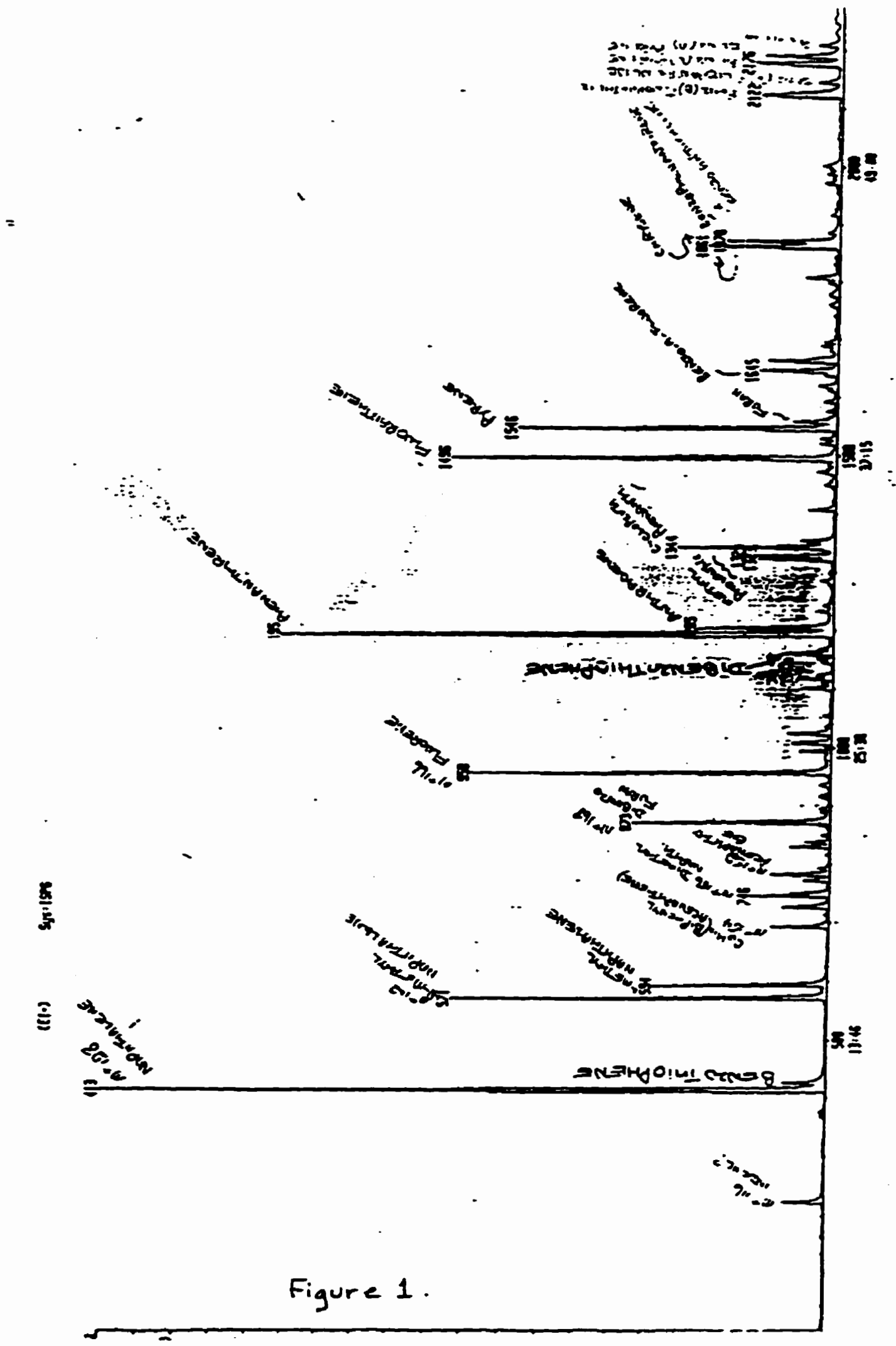




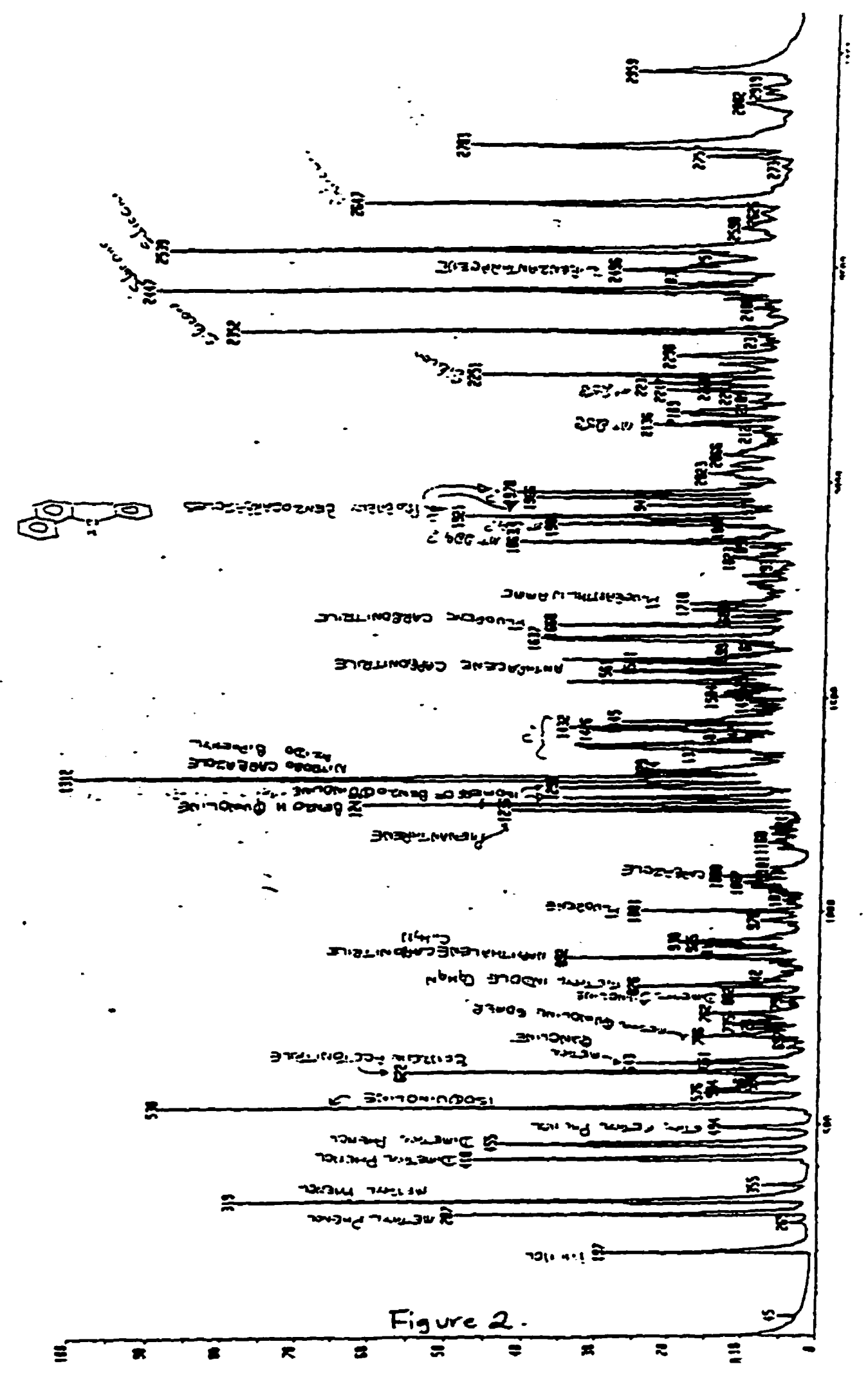




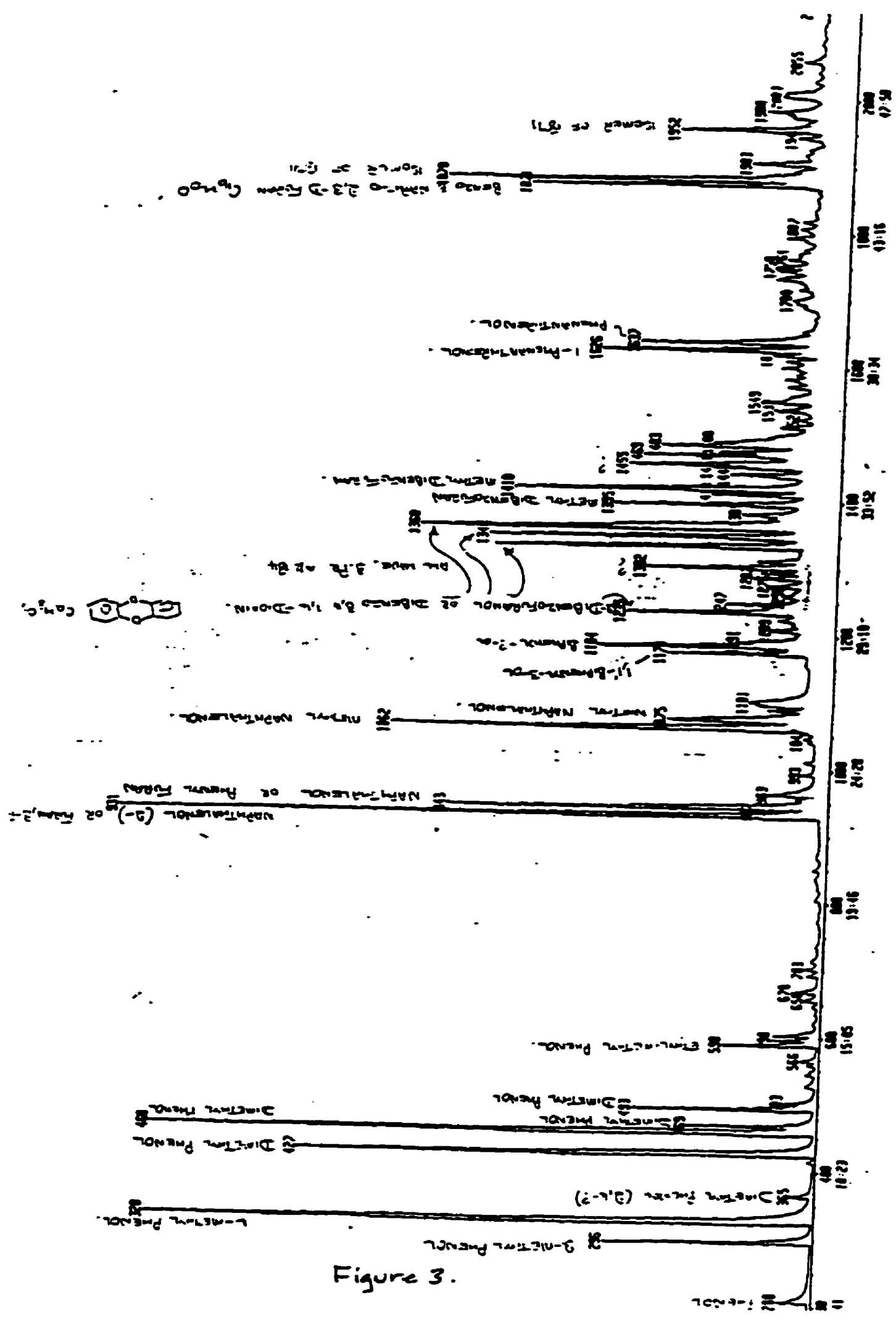


종로

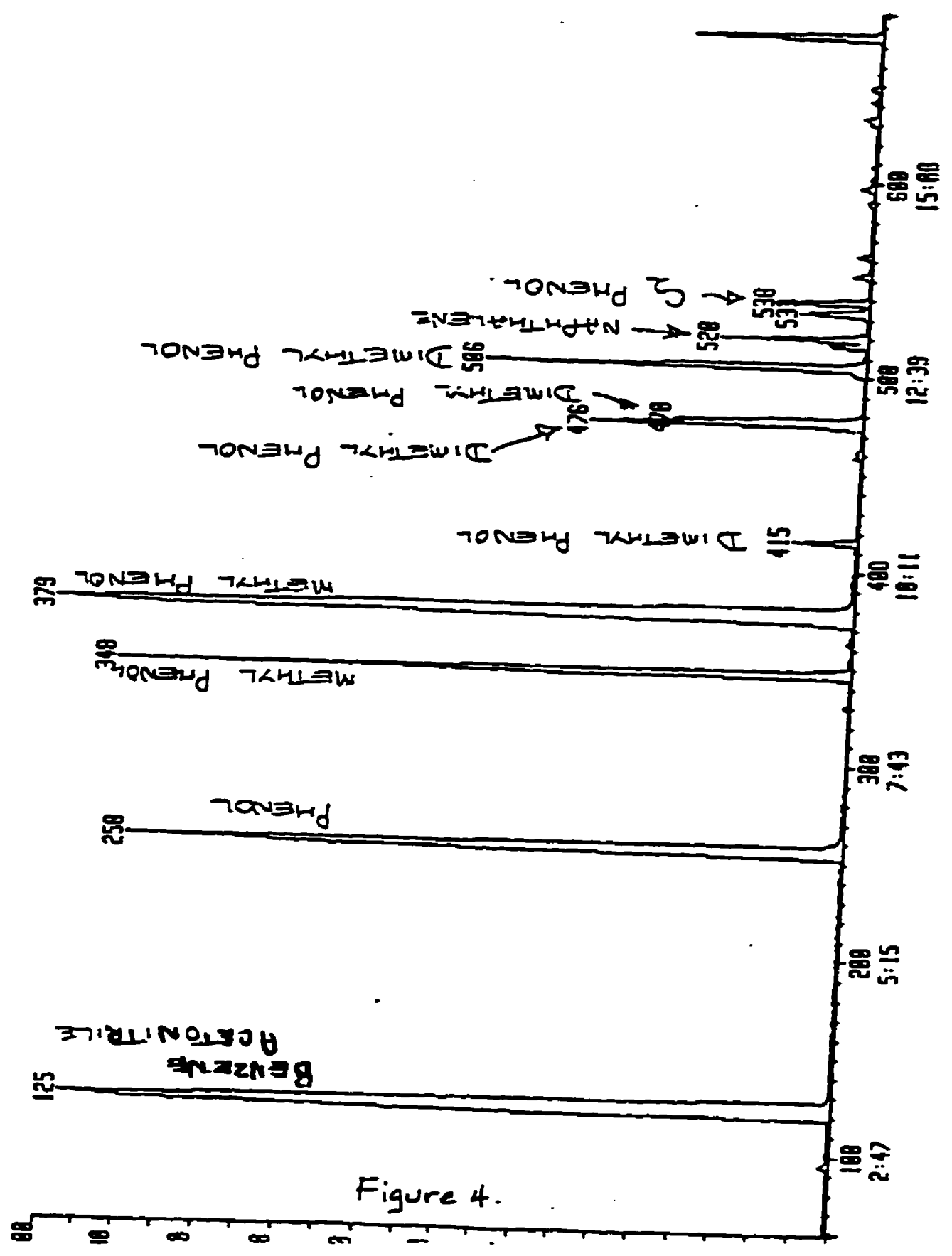




\section{APPENDIX 6 \\ Paper Presented at the APINGWA Conference \\ November, 1994, Houston}




\title{
Groundwater Plume Development from a Complex Organic Mixture
}

\author{
Mark King', Heather Malcolmson ${ }^{2}$ and James Barker' \\ 'Waterloo Centre for Groundwater Research \\ 2 Gartner Lee Limited
}

\begin{abstract}
Creosote is a complex mixture of polycyclic aromatic hydrocarons (PAHs), heterocyclic compounds and phenolic compounds. The fate of plumes originating from mixtures of this type is complicated since the solubility and environmental persistence of the constituent compounds can vary greatly. The purpose of the present study is to evaluate the applicability of Raoult' $s$ law as a dissolution model for creosole at a field research site. In the laboratory component of this work, batch tests were conducted to evaluate the ideality of creosote. Batch results were then used to calculate expected initial concentrations of creosote compounds at a field site where a volume of soil containing coal tar creosote was emplaced below the water table. Transfer of organic compound mass from creosote to groundwater was characterized with intensive sampling of the developing plume and the dissolving source.
\end{abstract}

Results indicated that Raoult's law provides a reasonable prediction of batch test concentrations for 4 of the 10 compounds evaluated. A dissolution model based on Raoult's law and the concentrations from the batch test also provided a reasonable predietion of dissolution from the field source for three of four compounds evaluated. It was noted that throughout the source, concentrations of the more soluble compounds ( $m$-xylene and naphthalene) became more variable with time while the degree of variability remained constant for the less soluble compounds (dibenzofuran and phenanthrene). The evolving ratios between compounds were predictable with the Raoult's law model. However it was necessary to extend the model well past the duration of the field experiment in order to fit all the data. This was taken as an indication that creosote was heterogeneously distributed in the original source. The increase in variability of the more soluble compounds with time has implications for evaluating mass flux from complex organic sources at contaminated industrial sites. 


\section{INTRODUCTION}

Many products and wastes derived from coal and oil contain varying amounts of PAHs. heterocyclic compounds and phenolic compounds. Examples of these types of materials include coal tar, creosote and petroleum sludges. They are often complex in composition. with constituents that vary widely in aqueous solubility. As a result, a complex groundwater plume may evolve if these types of mixtures are allowed to enter the environment. Typically. it is difficult to predict the resulting groundwater composition since the complete composition of the parent material is often not known. Also, even if the parent material is fully characterized the equilibrium solubilities of the constituent compounds may not be predietable by the simplifying assumption of ideal behoviour. Since many of these compounds are aerobically biodegradable (Meuller et.al. 1989) a first step in evaluating the environmental risk associated with a contamination occurrence may involve investigation of the expected degree natural attenuation (Salanitro. 1993). In erder to do this, it is necessary to evaluate the mass flux of contaminants to groundwater.

The research discussed in this paper is part of the Coal Tar Organies in Groundweter Program at the Waterloo Centre for Groundwater Research and is ongoing. The objective of the overall program is to conduct research on the environmental behaviour of mixtures of coal tar organies, specifically, PAHs, heterocyclic compounds and phenolic compounds. In this paper we present results from a laboratory batch dissolution test and from an emplaced creosote source at a field site. Creosote was selected as the source material because it is comprised of a wide range of PAHs, heterocyclic and phenolic compounds that are found in other coal- and oit-derived mixtures. In the following, we compare laboratory and field dissolution results with predietions according to Raoult's law and provide preliminary conclusions. The purpose of this comparison is to evaluate the validity of applying Raoult law as a dissolution model for creosote at field research site and other sites where groundwater is affected by similar mixtures. For the research site, this work will be used to estimate the mass flux of creosote compounds to groundwater so that the rate and processes of natural attenuation can be evaluated.

\section{BACKGROUND}

\section{Creosote Properties-and Environmental Significance}

Creosote is used as an industrial wood presenvative and is a thin oily liquid that may vary from yellow-green to black in colour. Since it has a density slightly greater than that of water and is composed of hydrophobic compounds it is classitied as a dense nonaqueous phase liquid (DNAPL). In general, creosote may consists of up to 200 chemicals (Meuller et.al., 1989): approximately $85 \%$ PAHs, $10 \%$ phenolic compounds and $5 \%$ oxygen-, sulphur-, and nitrogenheterocyelie compounds. The solubility of the components varies by several orders of magnitude.

In Canada, it is reported that creosote contemination of soils, groundwater and surface waters is known to be occuming at 24 past or present creosole-handling sites (Environment Canada. 1993). There are currently 27 ereosote wood treatment facilities in operation in the country. It is estimated that almost 1400 wood treatment facilities. half of which are inactive. exist in the United States (USEPA, 1992). More than 60 of these sites are on the USEPA National

Priorities (Superfund) List. 


\section{Dissolution}

When a compound partitions between two phases to the degree that equilibrium is reached the chemical activities for the compound are, by definition, equal in both phases such that (Lane and Loehr, 1992):

$a_{1}=x_{1}^{*} b_{1}^{2}=x_{1}^{0} b_{1}^{0}$

where $a$, is the activity of solute $i$, the superseripts a and 0 signify the aqueous and organic phases, respectively, $x$, is the mole fraction of $i$ in exch phase and $b_{b}$ is the activity coeficient of $i$ in each phase. A simplification of this equilibrium relationship is often applied on the basis of Raoult's law. This law wes originally used to define the linear relationship between partial pressure of a component in the heedspace over a multi-component liquid and the mole fraction of that component in the liquid (Laidler and Meiser, 1982). In terms of partial pressure, a liquid is termed "idear" if it conforms to this linear relationship. For the extension of Raoult's law to partitioning between an aqueous phase and an organic mixture, components in the mixture are said to display ideal behaviour if they partition linearly to the aqueous phase in proportion to the product of their mole frastion in the organic phase and their mole fraction solubility.

Two assumptions are inherent in this approach (Mackay. et.al. .1991): 1) the activity coefficient in the organic phase is equal to unity - a reasonable approximation for organic mixtures composed of compounds with similar chemical properties and 2) the inverse of the solute activity coefficient is equal to the mole fraction solubility of the component. Incorporating these two assumption into equation 1 gives the mole fraction form of Raoult's law:

$x_{1}^{*}=x_{1}^{0} x_{i}^{*}$

where $X_{i}^{\prime}$ is the mole fraction solubility of for the pure component in contact with water. Rewriting equation 2 in terms of the concentration gives (Shiu et.al., 1988):

$C_{1}^{\prime}=x_{1}^{0} S_{1}$

where $C_{1}$ is aqueous concentration and $S_{1}$ is the pure component liquid solubility. For compounds that are solid in pure form and at the temperature of the given system, liquid phase solubility can be calculated with the following (Shiu et.al., 1988):

$S_{1}=S_{s} \exp \left[6.8\left(T_{\mu} / T-1\right)\right]$

where $S_{3}$ is solid solubility, $T_{m}$ is melting point $\left({ }^{\circ} \mathrm{K}\right)$ and $T$ is the system temperature. For compounds that are solid at the system temperature, the calculated liquid solubility will be greater than the solid solubility but, as pointed out by Mackay et.al. (1991), the dissolved concentration can never exceed the latter. Values of these parameters for the compounds selected for this study are provided in Table 1.

Several studies have examined the degree to which various compositionally complex organic mixtures conform the Raoult's law model of ideal behaviour. Cline et.al. (1991) determined, through batch-type testing, that a variety of $\mathbf{3 0}$ gasolines conformed closely to Raoult's law. Aqueous partitioning of eight PAHs from four different diesel fuels was investigated with batch tests by Lee et.al. (1992a) and determined to be well described (within a factor of two) by 
Table 1. Creosote compound properties and initial concentrations

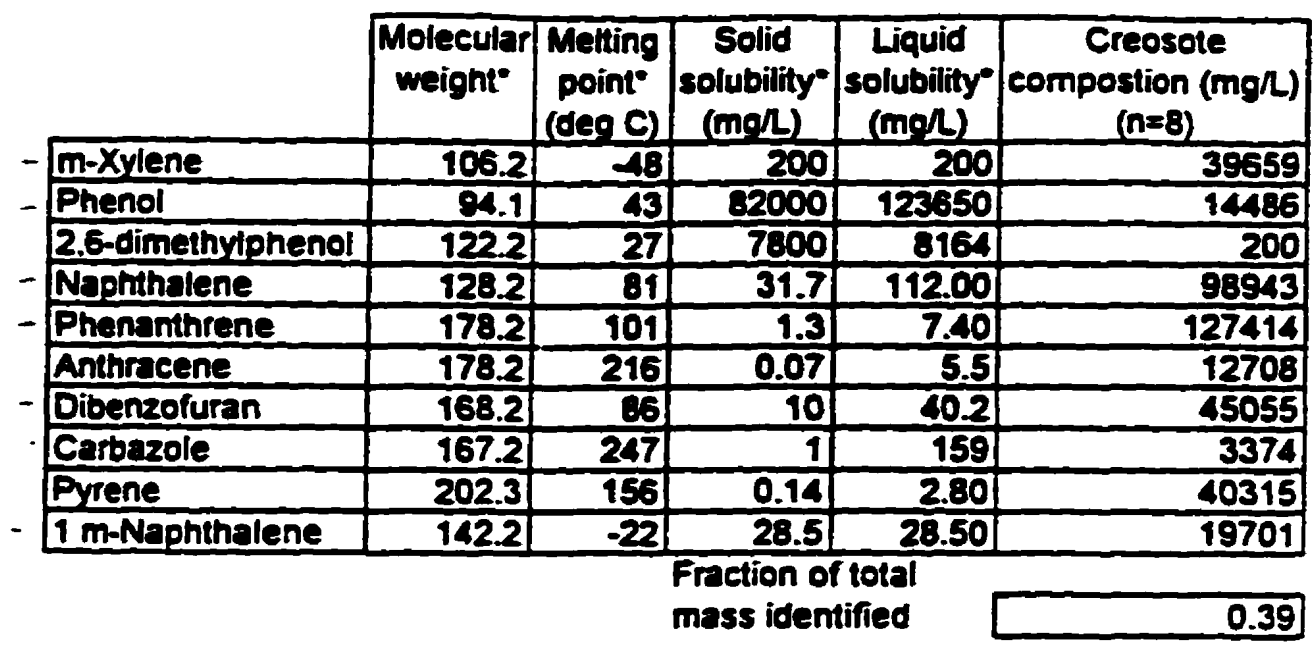

- Information sources: WCGR. 1992 and Versehueren, 1983.

-* all analysis performed after amendment with additional compounds (m-xylene. carbazole, p-cresol and phenol)

assuming ideal behaviour. A similar degree of agreement was reponted by Lee et.al. (1992b) for partitioning of PAHs from eight different coal tar samples. also with batch tests. Priddle and MacQuarrie (1994) examined the solubility of ereosote with a dynamic column experiment. In a mass transfer rate test. five of the seven PAHs for which they analyzed conformed to ideal behaviour within a factor of two.

\section{Field Site}

The field research site is located at Canadian Forces Base (CFB) Borden, approximately 80 $\mathrm{km}$ northwest of Toronto. Ontario, Canada. It is in an unused sand pit situated approximately $350 \mathrm{~m}$ north of a municipal landfill that operated from 1970 to 1976. Several hydrogeological studies have been conducted at this location over the past 15 years. Geology and local-scale hydrogeology of the area was diseussed in detail by Macfarlane et.al. (1983). Mackay et.al. (1986) discussed the hydrogeology and groundwater quality in the sand pit area. Only details relevant to dissolution of the creosote source are discussed here.

The site is underlain by an unconsolidated sand aquifer consisting of medium- to fine-grained sand. These deposits are glaciolacustrine in origin and grade into sitts and clays at a depth of approximately nine metres. Macfarlane et. al. (1983) and Nicholson et. al. (1983) discussed the inorganic chemistry and migration of a groundwater leachate plume originating from the landill and underlying the sand pit study site at depths ranging from 6 to $7 \mathrm{~m}$. From the water table to the top of the leachate plume, groundwater is largely unaffected by the landfill. A study of the groundwater flow system in the sand pit was conducted by Linderfelt et.al. (1994) at the same time as the study reported herein. They reported that the water table in the sand pit ranges from the surface 10 a depth of approximately $1.5 \mathrm{~m}$. Estimates of hydraulic conductivity by Mackay et. al. (1986) indicated a mean value of approximately 
$7 \times 10^{-5} \mathrm{~m} / \mathrm{s}$. Mean porosity was estimated at 0.33 and the average linear velocity of groundwater was estimated at $0.091 \mathrm{~m} /$ day

Chemistry of background groundwater (unaffected by the leachate plume) at the site was examined by Nicholson et. al. (1983) and Mackay et. al. (1986). The groundwater is relatively hard with low dissolved organic carbon. Dissolved oxygen was determined to be variable but in general the aquifer was aerobic with oxygen ranging up to $8.5 \mathrm{mg} / \mathrm{L}$. Data collected through this study indicate that the dissolved oxygen concentration in groundwater directly upgradient of the creosote source is 2 to $3 \mathrm{mg} / \mathrm{h}$.

\section{METHODS}

\section{Sample Analysis}

The analytical methodology used for the study was developed for quantification of creosote compounds in groundwater, sand and pure creosote. Compounds selected for analysis were intended to be representative of the main groups of compounds found in creosote and included two phenolic compounds (phenol and 2,6-dimethylphenol), five PAHs (naphthalene, 1-methylnaphthalene, anthracene, pyrene and phenanthrene) and two heterocyclic compounds (carbazole and dibenzofuran). M-xylene was included in the analytical suite as a representative petroleum hydrocarbon. Analysis was also conducted for several other compounds but due to the variable availability of analytical standards, only the 10 compounds above were analyzed throughout the whole experimental period.

Groundwater samples (typically $45 \mathrm{~mL}$ ) were prepared by adding $9 \mathrm{~g} \mathrm{NaCl}$ to promote partitioning of all analytes and $1 \mathrm{~mL} 1 \mathrm{~N} \mathrm{HCl}$ to enthance partitioning of phenolic compounds. Samples were then capped and allowed to stand for 24 hours before addition of $3 \mathrm{~mL}$ dichloromethane. Samples were then placed. on a shaker for 20 minutes at 350-400 $\mathrm{pm}$ and $1 \mathrm{~mL}$ of the solvent was removed to an autosampler vial. Pure creosote was prepared for analysis by dissolving a $10 \mathrm{uL}$ sample in $10 \mathrm{~mL}$ dichloromethane and then transferring $1 \mathrm{~mL}$ to an autosampler vial.

Extracts were injected into a Hewlett Packard 5890 Gas Chromatograph (GC) equipped with an HP7673A autosampler and Flame lonization Detector (FID). The temperature program for analysis was as follows: $40^{\circ} \mathrm{C}$ for $0.5 \mathrm{~min}, 15^{\circ} \mathrm{C} / \mathrm{min}$ to $300^{\circ} \mathrm{C}$, hold $10 \mathrm{~min}$. The carrier gas was helium with a flow rate of approximately $24 \mathrm{~mL} / \mathrm{min}$. The injector temperature from the GC was $275^{\circ} \mathrm{C}, \mathrm{FID}$ temperature was $325^{\circ} \mathrm{C}$ and injection volume was $3 \mathrm{uL}$.

\section{Creosote Characterization}

Raw creosote was amended with certain compounds so it would be more widely representative of typical composition (Meuller, 1989). To $69.5 \mathrm{~kg}$ of creosote the following compounds were added: $0.45 \mathrm{~kg}$ carbazole, $0.50 \mathrm{~kg}$ p-cresol, $1 \mathrm{~kg}$ phenol. M-xylene was also added $(3 \mathrm{~kg})$. to provide a compound representative of petroleum hydrocarbon sites. The density of the modified creosote was $1.03 \mathrm{~g} / \mathrm{mL}$.

\section{Batch Dissolution}

A batch dissolution test was conducted to evaluate the effective solubility (Se) for selected compounds. Additional details of the experiment are provided by Malcolmson (1992). Into 
each of three glass crimp-top hypovials a quantity of creosote was added (between 4.21 and $6.51 \mathrm{~g}$ ) and the remainder of the bottle was filled with background Borden groundwater (between 62.28 and $72.66 \mathrm{~mL}$ ). The bottles were then sealed with teflon-lined caps and stored in the dark at approximately $10^{\circ} \mathrm{C}$ for one month. One water sample was removed from each bottle for analysis of equilibrium aqueous concentration and duplieate samples of creosote were removed from each bottle for analysis of equilibrium creosote concentrations.

\section{Field Source Emplacement and Monitoring}

The creosote source was emplaced hydraulically upgradient of an existing multi-level bundle piezometer network. The system was first used for the joint Stanford-Waterloo natural gradient tracer test conducted from 1982 to 1985 (Mackay et.al., 1986). The network has been modified and added to in subsequent studies, including this one, and the current layout of the near-source piezometers in relation to the creosole source is shown in Figure 1. Since this paper is concemed only with date collected within the source, subsequent description will focus on methodology directly related to the source.

The creosote source, shown in Figure 2, was emplaced on August 28, 1991. Details of the source installation are provided by Malcolmson (1992). Sealable sheet piling (Starr et.al.. 1991) was vibrated into the ground in a rectangular array $(5 \times 1.5 \mathrm{~m})$. The sand inside the sheet piling was excavated after it was dewatered with two shallow dewatering wells. The source sand was mixed with creosote in a cernent mixer at less than $5 \%$ creosote by volume: laboratory testing had indicated that it should be effectively immobile at this concentration. A total of approximately $74 \mathrm{~kg}$ of creosote was added to approximately $5800 \mathrm{~kg}$ of sand.

Borden sand was not used for the source material since the addition of creosote to the sand would result in some decrease in porosity which would inhibit the movement of groundwater through the source. Instead a coarser sand was used, with a hydraulic conductivity of approximately $3.59 \times 10^{-4}$. based on sieve analyses (Devlin, 1994) - approximately 5 times greater than Borden sand. Porosity of the source sand was estimated at 0.31 (Malcolmson. unpublished data) and it was calculated that with the creosote addition the porosity would effectively decrease to approximately 0.29 . Work in progress will provide a detailed evaluation of groundwater flux through the source. However, preliminary hydraulic modelling indicates that the flux rate through the source should be similar to the ambient groundwater flux rate in the vicinity of the monitoring system. On that basis, a total now rate through the source of $0.204 \mathrm{~m}^{3} /$ day $\left(0.030 \mathrm{~m}^{3} / \mathrm{day} / \mathrm{m}^{2}\right.$ cross-sectional area) was used as an estimate of the amount of groundwater contacting the source.

Bundle piezometers were installed within the source at locations shown in Figure 2. Each piezometer consisted of either 11 or 13 monitoring points, separated by a vertieal distance of 20 to $30 \mathrm{~cm}$ and construeted of $3 \mathrm{~mm} 10$ stainless steel tubing. To install the piezometers, a $5 \mathrm{~cm}$ ID aluminum tube fitted with an aluminum tip was vibrated into the.ground with a vibrating hammer. The tip was then knocked out of the end of the aluminum tube and the bundle was inserted. The aluminum tube was then pulled out of the ground allowing the sand to collapse around the bundle.

Samples have been collected from a few selected piezometers within the source on an approximately monthly basis, up to the present. In addition, more intensive "snapshot" sampling of approximately 30 points within the source, was conducted several times since the source was emplaced. In this paper we will discuss a time series of samples collected from 


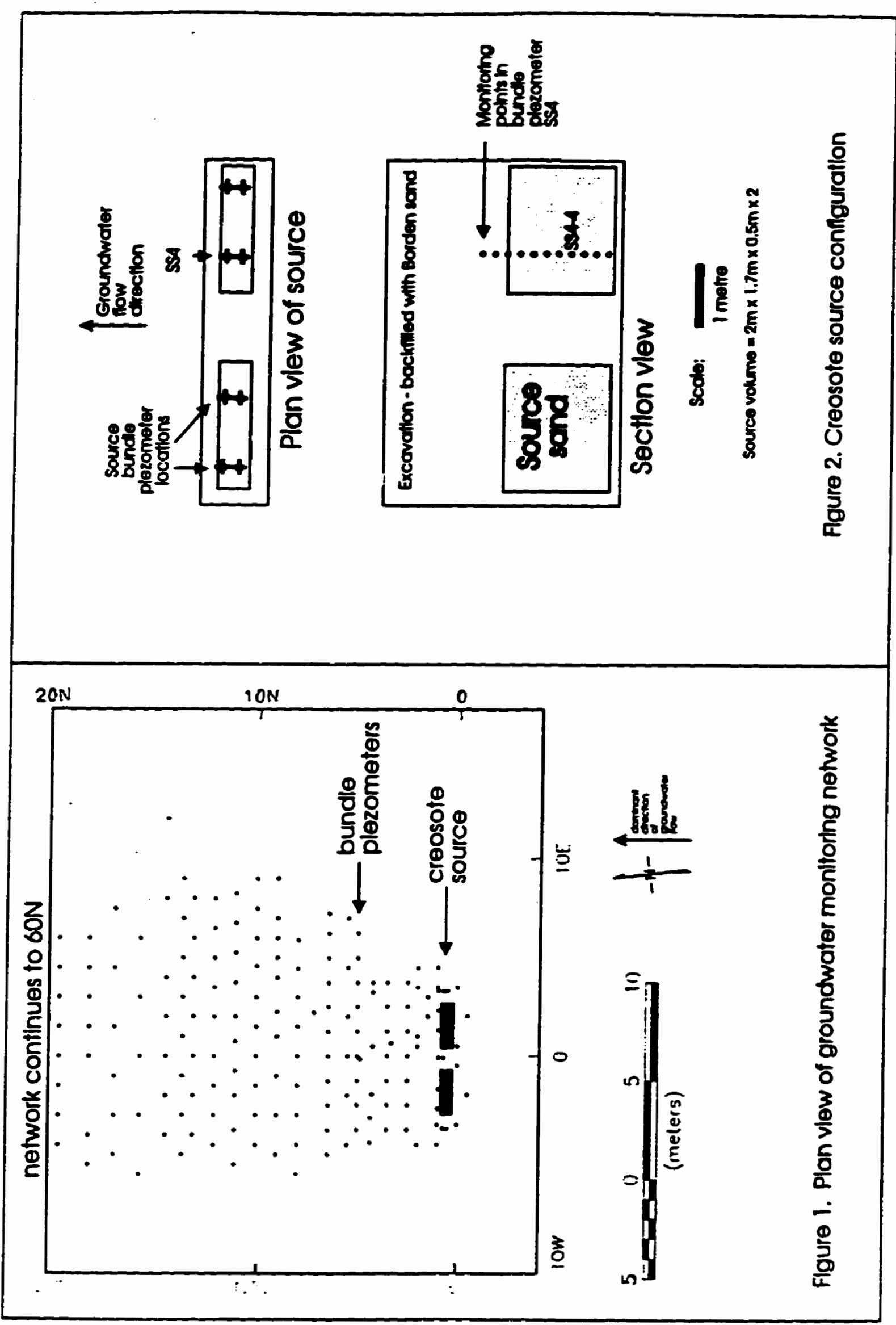


one point (SS4-4 shown on Figure 2) and two sets of snapshot samples collected at 55 and 1008 days. All groundwater samples were collected in $60 \mathrm{~mL}$ hypovials using a suction sampling head. This device is fitted to the top of the sample bottle and contains two ports: an outlet port attached to a suction pump and inlet port attached to a piezometer tube. When a vacuum is drawn on the outlet. groundwater is drawn in through the inlet. At least one tubing volume was purged before sample collection. Additional details on sample collection are provided by Malcolmson (1992) and Nielsen and Hansen (1992).

\section{RESULTS AND DISCUSSION}

\section{Creosote Characterization}

Averages of analytical results for eight samples of the altered creosote (analyzed prior to field source emplacement and after amendment with four additional compounds) are shown in Table 1.

\section{Batch Test}

Results of batch testing were used to calculate $S_{1}$ by rearranging equation 3 . The creosote mole fractions used in this case were those calculated for creosote from the batch test bottles at the end of the equilibration period. A molecular weight of 200 was assumed for the unidentified portion of the creosote. Values for $C_{i}^{;}$were taken directly from analysis of the water. Calculated $S_{1}$ values are shown in Table 2 and are compared with literature values (or those calculated with equation 4 , as required).

Comparison of these two values gives some measure of the ideality of the creosote in terms of dissolution behaviour. Lee et.al. (1992a) use a "factor of two" as a guide for evaluating the agreement between Raoult's law predictions and measured solubility, and hence the ideality of the organic liquid. The ratios between $S_{i} s$ calculated from the batch test results and those from the literature are shown in Table 2. Raoult's law provides a satisfactory predietion (within a factor of (wo) of batch results for $m$-xylene, naphthalene and dibenzofuran but phenol, phenanthrene, carbazole and pyrene are overestimated. Since 2,6-dimethylphenol and anthracene were not detected in the aqueous samples, they were also overestimated. Only 1methylnaphthalene was significantly underestimated by Raoult's law.

\section{Field Results}

Results from batch testing were used as an updated estimate of $S_{1}$ for application to the field data. The product of the batch-derived $S$, and the initial creosote mole fraction (Table 2) was used as the Raoult's law prediction of expected initial effective solubilty (Se) from the freld creosote source. Conceptually, the Se determined in this manner can be considered the expected concentration if a small volume of water contacted a much larger volume of creosote such that the mass loss from the organic phase does not cause a significant change in mole fractions, even for the most soluble compounds. Mole fractions of the initial creosote were again calculated using an assumed average molecular weight of 200 for the unidentified portion of the creosote. Estimates of initial Se (aqueous concentrations in equilibrium with initial creosole) are shown in Table 2. 
Table 2. Comparison of equilibrium aqueous concentrations from literature and application of Raoult's law to batch dissolution results

\begin{tabular}{|c|c|c|c|c|c|}
\hline & \multirow{2}{*}{$\begin{array}{l}\text { Creosote } \\
\text { mole } \\
\text { fractions" }\end{array}$} & \multicolumn{2}{|c|}{ Pure compound solubility (mg $\Omega$} & \multirow{2}{*}{$\begin{array}{l}\text { Ratio } \\
\text { (1) : (2) }\end{array}$} & \multirow{2}{*}{$\begin{array}{c}\text { Aqueous } \\
\text { concentration } \\
\text { in equlibrium } \\
\text { with creosote (mgn) }\end{array}$} \\
\hline & & $\begin{array}{c}\text { (1) } \\
\text { literature } \\
\text { values }\end{array}$ & $\begin{array}{c}\text { (2) } \\
\text { Batch dissolution } \\
\text { lests (n=3) }\end{array}$ & & \\
\hline$m$-xylene & 0.57 & 200 & 225 & 0.9 & 14.4 \\
\hline Phenol & 0.15 & 123650 & 52787 & 2.3 & 1388 \\
\hline 2.6-dimethylphenol & 6.0. & 8164 & not detected & - & $\overline{0}$ \\
\hline Naphthalene & 0.77 & 112 & 89 & 1.2 & 12.4 \\
\hline Phenanthrene & 0.72 & 7.4 & 3.6 & 2.1 & 0.44 \\
\hline Anthracene & 0.07 & 5.5 & not detected & $=$ & $\overline{0}$ \\
\hline Dibenzofuran & 0.27 & 40.2 & 33.9 & 1.2 & 1.6 \\
\hline Carbazole & 0.02 & 159 & 67 & 2.4 & 0.23 \\
\hline Pyrene & 6.1. & 2.80 & 0.98 & 2.9 & 0.03 \\
\hline $1 \mathrm{~m}$-Naphthalene & 0.14 & 28.5 & 71.0 & 0.4 & 1.7 \\
\hline
\end{tabular}

Figure 3 depicts the evolution of Se, based on Raoult's law, for selected creosole compounds ( $m-x y l e n e$, naphthalene, dibenzofuran and phenanthrene) dissolving from the emplaced creosote source. These curves were calculated with a simple spreadsheet model using the assumptions stated above and also assuming that the unidentified portion was insoluble. The value used for groundwater flux through the source was $0.204 \mathrm{~m}^{3} / d a y$, for reasons discussed earlier. For the purposes of the calculations it was assumed that all the groundwater passing through the source during a given time step reaches equilibrium with the creosote, according to equation 3. At the beginning of the next time step, the mass dissolved into the aqueous phase during the previous step was subtracted from the creosote and mole fractions were recalculated. Calculations for the first 100 days were performed with a relatively short time step ( 0.25 days) to minimize the error of this approximation during the time that phenol mole fraction is changing rapidly. The time step was subsequently increased to 5 days since the remaining analyzed compounds are less sensitive to this type of error due to their relatively low solubilities.

Also shown on Figure 3 are groundwater concentrations at level 4 of multi-level piezometer SS4, installed within the creosote source. The location of this monitoring point is shown on Figure 2, denoted as SS4-4. This level was selected for demonstration because it has been monitored consistently and has maintained relatively consistent concentrations. In some other monitoring points small blobs of pure phase creosote have occasionally been recovered during sampling and have caused an apparent, but erroneous, increase in dissolved concentrations. The model based on Raoult's law approximates most of the field data within a factor of two, although considerable scatter is apparent in the measurements. The significant decreases in concentration predicted over the monitoring period for $m$-xylene and naphthalene are evident in the measured data. Dibenzofuran deviates most from the predicted behaviour. Nonideality of the creosote is not considered to be the cause of the 
difference since the pure compound liquid solubility used to calculate the initial Se was determined through batch testing. Rate-limited dissolution (ie., limited by contact time belween groundwater and source creosote) is also not suspected as the cause since it does not appear to have limited concentrations of the other three compounds. although it cannot be ruled out. Potential causes under review include analytical uncertainty in characterization of the initial creosote (eg., co-elution of an unidentified compound with dibenzofuran) and the batch test samples.

Figure 4 shows concentration profiles in SS4 for m-xylene, naphthalene, dibenzofuran and phenanthrene at 55 days and 1008 days after emplacament of the ereosote source. M-xylene and naphthalene show more variable depletion between sample sets. This increase in variability of aqueous concentrations with time, which is best illustrated by the naphthalene profiles, may result from heterogeneous distribution of creosote mass in the source. Care was taken during installation to kecep the creosote-sand mixture consistent. However, cores

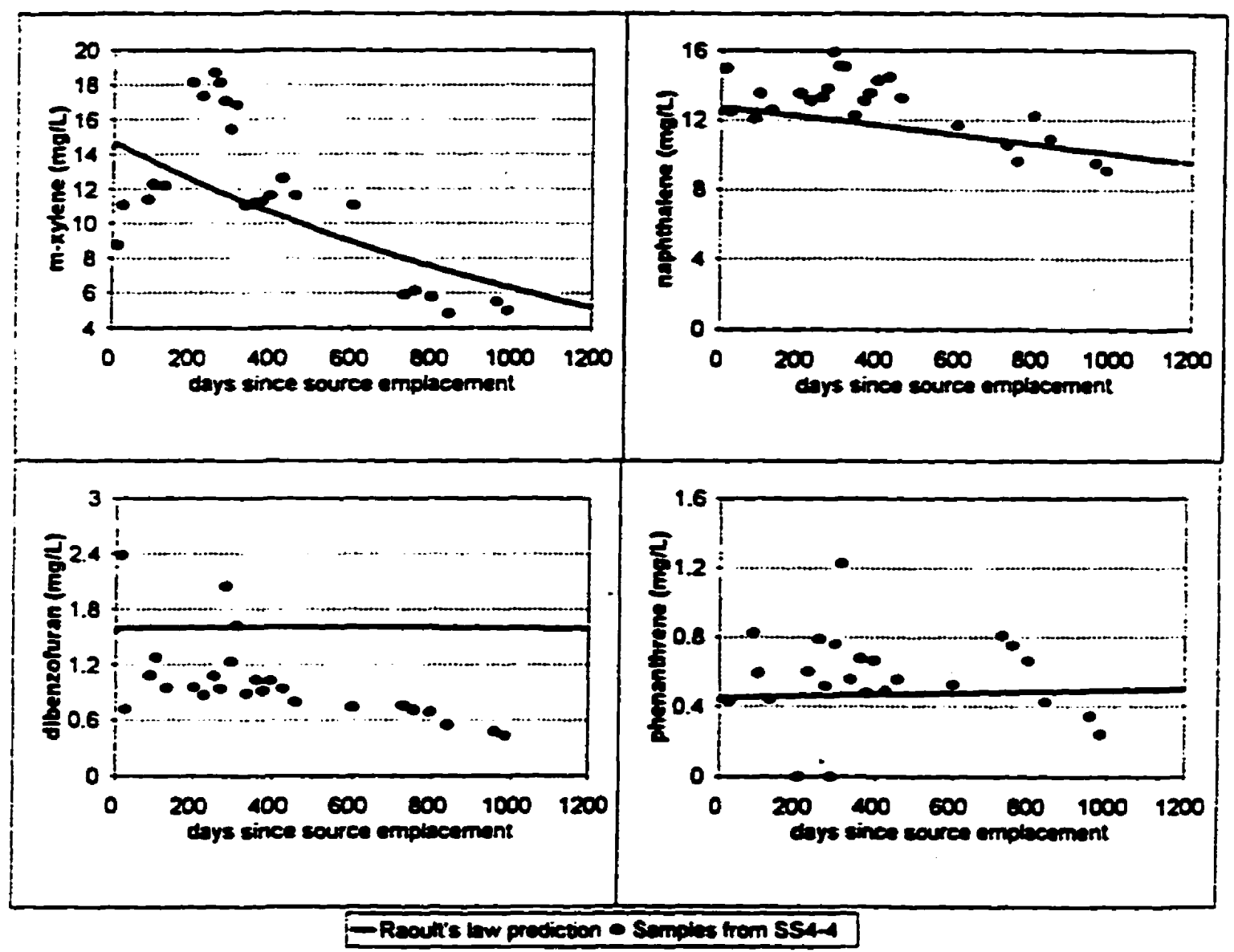

Figure 3. Comparison of dissolved concentrations of creosote compounds ( $m$-xylene. naphthalene. dibenzofuran and phenanthrene) in groundwater within the creosote source with concentrations predicted by applying Raoult's law; values for pure compound liquid solubility have been determined with batch dissolution tests. 
collected from the source shortly after emplacement indicated considerable variability in creosote concentration (data not shown). In portions of the source that contained less creosote mass, higher solubility compounds such as naphthalene and $m$-xylene may have been depleted as indicated by aqueous concentrations that are much lower than expected in some locations. Less spreading was observed for dibenzofuran and phenanthrene concentrations, probably as a result of lower solubility. However, an overall decrease in dibenzofuran was observed and is difficult to explain since the predicted Se stays constant over this period. The obvious decrease in the measured values may indicate that the mole fraction in the initial creosote was less than that indicated by analysis.

A further illustration of increasing variability in aqueous concentrations of the more soluble compounds is shown in Figure 5. The measured data are from samples collected at points throughout the creosote source at 55 days and 1008 days. As shown in the plot of naphthalene vs. dibenzofuran, concentrations of both compounds showed relatively little

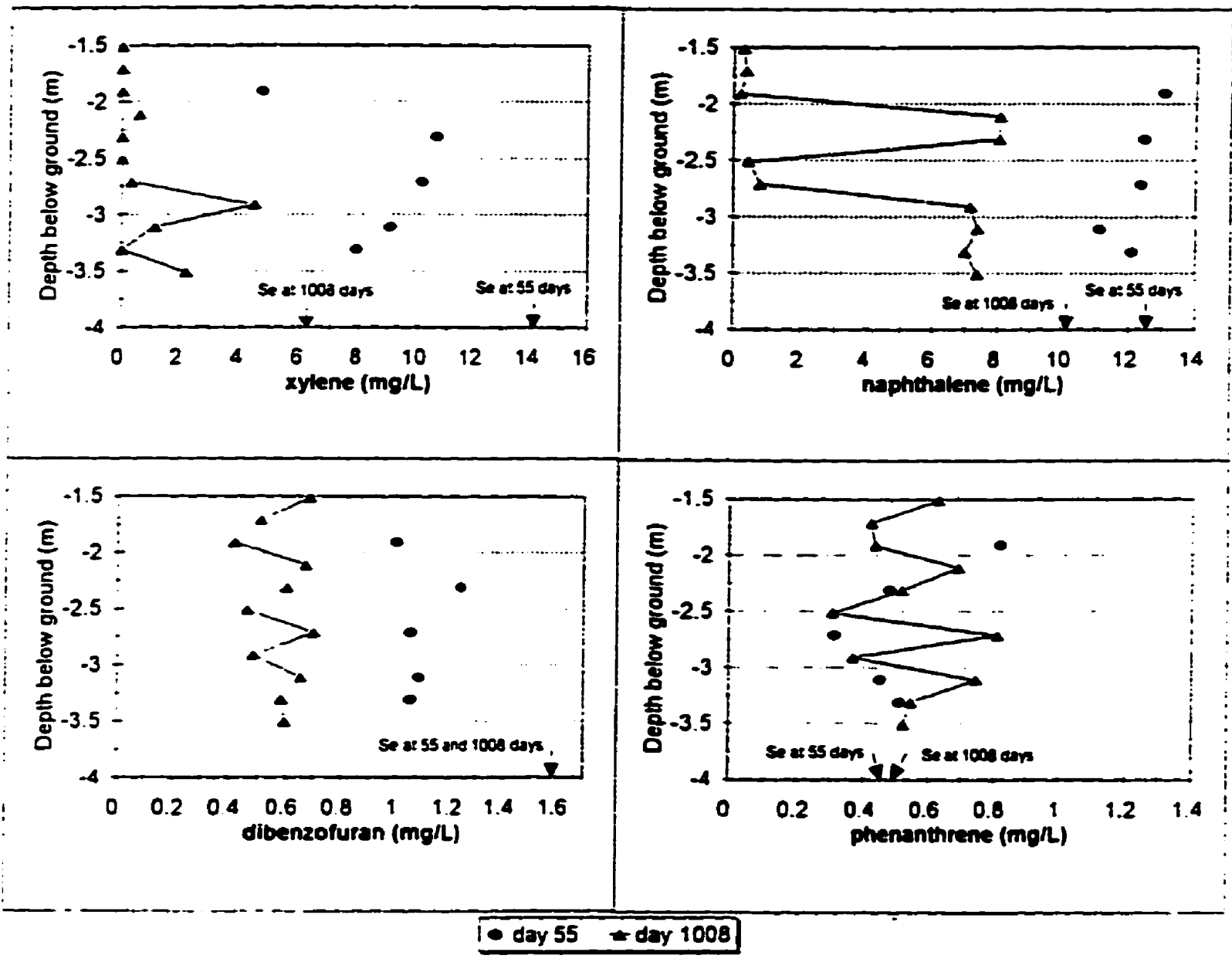

Figure 4. Concentrations of selected creosote compounds in multi-level piezometer SS4 at 55 days and 1008 days after source emplacement: effective solubilities (Se) shown are as predicted by Raoult's law (location of SS4 shown on Figure 2). 
variation throughout the source at 55 days. However, a considerable spread in the naphthalene data became apparent by 1008 days. Dibenzofuran concentration decreased but in a relatively consistent manner. A similar trend is noted for naphthalene vs. phenanthrene. with the exception that no decrease is apparent for phenanthrene after 1008 days.

Figure 5 also shows expected trends of compound ratios according to the Raoult's law model. These have been extended to 10,000 days to cover the range of measured concentrations. As expected, the data for dibenzofuran does not fit the predietion: again it appears that a lower initial mole fraction is indieated for dibenzofuran. However, data for naphthalene vs. phenanthrene and naphthalene vs. m-xylene show a reasonable agreement with the curves. with considerable seatter noted. At 55 days, most of the data points are located in a cluster at the early time part of the curves. However, by 1008 days the data are distributed along most of the length of the curves. Consequently, within the confines of the parameters used in the model, up to 10,000 days would be required for naphthalene and m-xylene concentrations to

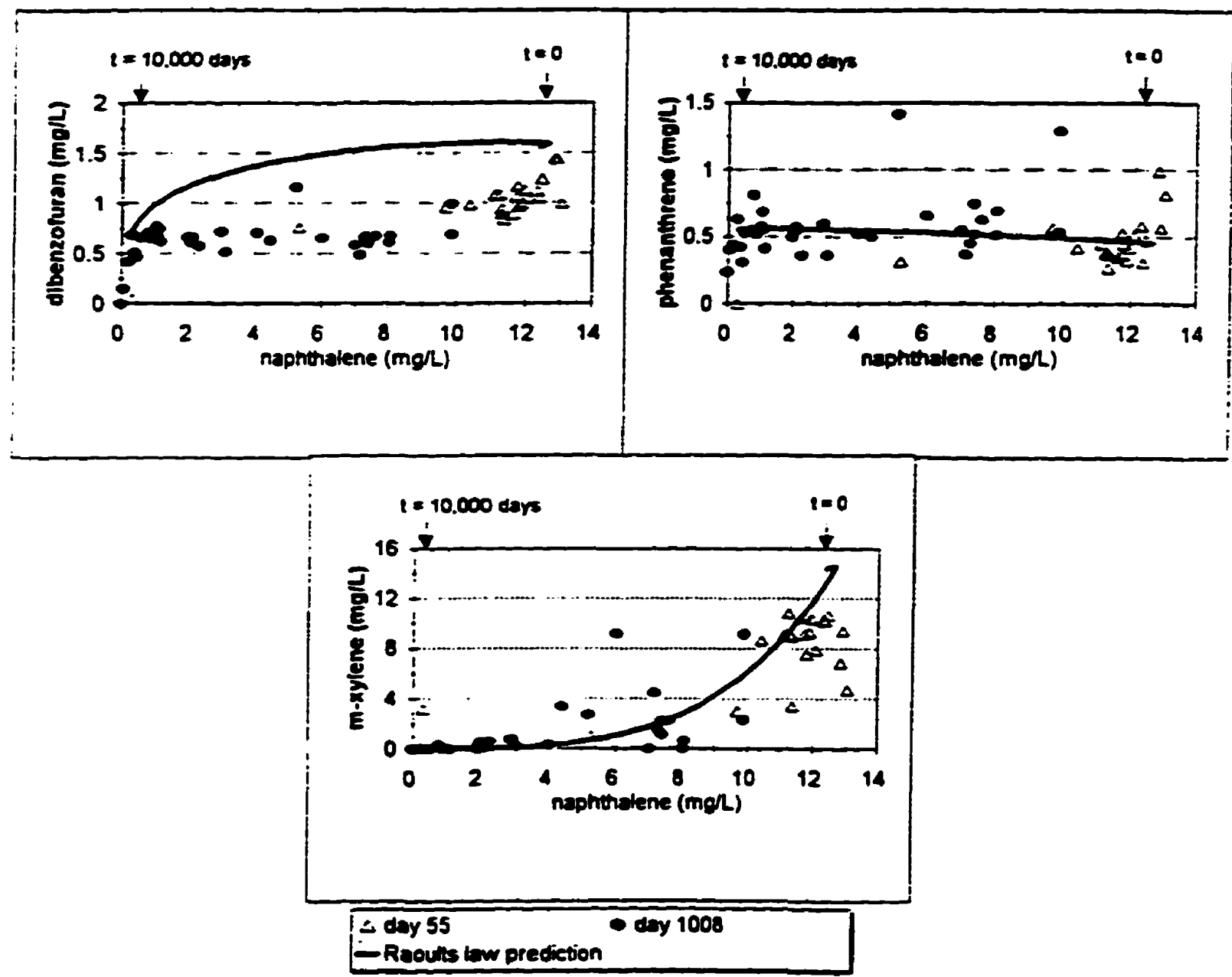

Figure 5. Dissolved naphthalene concentration vs. dibenzofuran. phenanthrene and $m$-xylene in groundwater throughout the creosote source; 55 days and 1008 days after source emplacement: duration of the Raoult's law model simulation is from 0 to 10.000 days (note: the small bend at right end of each curve is due to rapid depletion of the phenol mole fraction). 
decrease as low as those measured in many of the 1008 day samples. This result is consistent with the suggestion of variable initial concentrations of creosote throughout the source, since the effect of lowering the initial concentration is similar to that of extending the period of dissolution. The observation that the more soluble compounds have been depleted by as much as ten times sooner than predicted may therefore indicate that at some locations. initial creosote concentration was an order of magnitude less than expected.

It is notable that with the processes indicated above, it is possible for groundwater migrating out of a contaminant source area to have consistent composition initially even though the NAPL distribution is heterogeneous. With time, zones of low NAPL concentration could become depleted of the more soluble compounds. Composition of groundwater leaving the source would then become variable with respect to the more soluble compounds while concentrations of the less soluble compounds remain relatively consistent. This compositional variability may be important in terms of estimating dissolved mass flux from organic mixtures with a wide range of compound solubilities. On an actual contaminated industrial site, where contact time will typically be much longer, groundwater plumes evolving from these types of mixtures are likely to be even more variable in cross-section: more time is available to magnify the effects of heterogeneous NAPL phase distribution. In such a circumstance, considerable care is required to make a reasonable estimate of contaminant mass flux from a source area into a groundwater plume.

\section{CONCLUSIONS}

Results of dissolution batch tests showed that Raoult's law provided a reasonable estimate (within of factor of 2) of the effective solubility of four of ten analyzed creosote compounds. The effective solubility of m-xylene, naphthalene and phenanthrene in groundwater at one point in the emplaced creosote source was satisfactorily predicted by Raoult's law over a period of 1000 days, but dibenzofuran behaviour was not well predicted. The deviation for dibenzofuran is considered to be related to experimental methodology rather than nonideality of the creosote with respect to this compound.

The degree of variability in groundwater composition throughout the creosote source increased dramatically in a relatively short time for $m$-xylene and naphthalene. It is evident that at many individual points the Raoult's law model would not have provided a satisfactory prediction. Comparison of the Raoult's law model to groundwater concentrations throughout the source provided an indieation that the increasing variability was due to heterogeneous initial distribution of pure phase creosote. This observation has implications for interpreting organic plume data at sites contaminated with complex organic mixtures with a wide range of compound solubilities. 


\section{ACKNOWLEDGEMENTS}

This work was conducted as part of the Coal Tar Organies in Groundwater Program with funding from the Natural Sciences and Engineering Research Council of Canada and Ontario Ministry of the Environment. The efforts of Kim Hamilton in laboratory and field activities have been instrumental over the entire duration of the project and are gratefully acknowledged.

\section{BIOGRAPHICAL INFORMATION}

Mark King is presently a Ph.D. student at the University of Watertoo. His doctoral research involves investigation of the formation and biodegradation of plumes frem complex hydrocarbon sources. Before retuming to university he was a hydrogeologist and project manager at Jacques Whitford Environmental Limited where he was pimarily involved in assessment and remediation of petroleum hydrocarbon contamination. Address: Waterloo Centre for Groundwater Research, B.F. Goodrich Building. University of Waterloo, Watertoo. Ontario, Canada, N2L 3G1. Phone:(519) 885-1211, Ext. 2973 Fax:(519) 725-8720.

Heather Malcolmson completed an M.Sc. at the University of Waterioo in 1992 . The topic of her research was the dissolution of coll tar creosote from a source emplaced below the water table. Since graduation she has worked as a hydrogeologist with Gartner Lee Limited. Address: Gartner Lee Limited, 140 Renfrew Drive, Suite 102, Markham, Ontario, Canada, L3R 683. Phone: (905) 477-8400. Fax:(905) 477-1456.

Jim Barker is a professor at the University of Waterioo and Associate Director of the Waterioo Centre for Groundwater Research. His main research interests pertain to the behaviour of organic contaminants in groundwater including microbial processes. Dr. Barker is supervising research at a number of field sites and has had involvement in the development of groundwater monitoring devices and in situ biorestoration techniques for organic contaminants. Address: Waterloo Centre for Groundwater Research, B.F. Goodrich Building. University of Waterloo, Waterloo, Ontario, Canada, N2L 3G1. Phone:(519) 885-1211. Ext. 2103. Fax:(519) 725-8720.

\section{REFRENCES}

Cline, P.V., J.J. Delíno and P.S.C. Rao, 1991. Pantitioning of aromatic constituents into water from gasoline and other complex solvent mixtures. Environmental Science and Technology, v. 25. p. 914-920.

Devlin, J.F, 1994. Enhanced in situ biodegradation of carbon tetrachloride and trichloroethene using a permeable wall injection system. Ph. D. Thesis, University of Waterloo, Waterioo. Ontario.

Environment Canada, 1993. Creosote-impregnated waste materials. Priority substance list assessment report.

Laidler, K.J. and J.H. Meiser, 1982. Physieal Chernistry. Benjamin/Cummings, Menlo Park. CA.

Lane. W.F. and R.C. Loehr, 1992. Estimating the equilibrium aqueous concentrations of polynuclear hydrocarbons in complex mixtures. Environmental Science and Technology, v. 26, no. 5. p. 983-990. 
Lee. L.S. M. Hagwall, J.J. Delfino and P.S.C. Rao, 1992a. Partitioning of polycyclic aromatic hydrocarbons from diesel fuel into water. Environmental Science and Technology, $v$. 26. no. 11, p. 2104-2110.

Lee, L.S. P.S.C. Rao and I. Okuda, 1992b. Equilibrium partitioning of polycyclic aromatic hydrocartons from coal tar into water. Environmental Science and Technology, v. 26. no. 11, p. $2110-2115$.

Linderfelt, W.R. and J.L. Wilson, 1994. Field study of capture zones in a shallow sand aquifer. In Proceedings of Transport and Reactive Processes in Aquifers, April 11-15, 1994, Zurich, Switzeriand.

MacFarlane. D.S.. J.A. Cherry, R.W. Giliham and E.A. Sudicky, 1983. Migration of contaminants in groundwater a landfill: a case study. Joumal of Hydrology, v. 63. p. 129.

Mackay, D., W.Y. Shiu, A. Maijanen and S. Feenstra, 1991. Dissolution of non-aqueous phase liquids in groundwater. Joumal of Contaminant Hydrology, v. 8. p. $23-42$.

Mackay D.M., D.L. Freyberg. P.V. Roberts and J.A. Cherry, 1986. A natural gradient experiment on solute transport in a sand aquifer 1. Approach and overview of plume movement. Water Resources Research, v. 22, no. 13, p. 2017-2029.

Malcolmson, H, 1992. Dissolution of an emplaced creosote source, CFB Borden, Ontario. Project Master's Report, University of Waterioo, Waterloo, Ontario.

Meuller, J.G., P.J. Chapman and P.H. Pritchard, 1989. Creosole contaminated sites - their potential for bioremediation. Environmental Science and Technology, v. 23, no. 10, p. 1197-1201.

Neilsen, J.S. and-S.S. Hansen, 1992. Fate and transport of creosote compounds in a sand aquifer at CFB Borden, Ontario, Canada. Project Master's Thesis, Technical Universily of Denmark.

Priddle, M.W. and K.T.B. MacQuarrie, 1994. Dissolution of creosote in groundwater: an experimental and modelling investigation. Joumal of Contaminant Hydrology, v. 15. p. 27-56.

Salanitro, J.P. 1993. The role of bioattenuation in the management of aromatic hydrocarbon plumes in aquifers. Groundwater Monitoring Review, Fall, p. 150-161.

Shiu. W.Y., A. Maijanen, A.L.Y. Ng and D. Mackay, 1988. Preparation of aqueous solutions of sparingly soluble organic substances: II. multi component systems - hydrocaron mixtures and petroleum products. Environmental Toxicology and Chemistry, v. 7, p. 125-137.

Starr, R.C., J.A. Cherry and E.S. Vales, 1991. Sealable joint sheet pile cutoff walls for preventing and remediating groundwater contamination. Ontario Ministry of the Environment Technology Transfer Conference. November 25-26, 1991. Toronto. Ontario.

USEPA, 1992. Contaminants and remedial options at wood preserving sites. EPA/600/R-92/182

Verschueren, K., 1983. Handbook of environmental data on organic chemicals. Van Nostrand Reinhold, New York.

Waterloo Centre for Groundwater Research, 1993. Organic properties database, version 3.0. University of Waterloo, Waterloo, Ontario. 


\section{APPENDIX 7 \\ RESULTS FOR QUALITY ASSURANCE AND CONTROL}

1. Effect of sample and extract storage time

2. Field blanks

3. Trip blanks

4. Effect of sorption to teflon sampler tubing

5. Influence of purge volume on groundwater composition 


\section{Effect of sample and extract storage time on analytical chemistry results}

\section{Snapshot \#5, Emplaced Creosote Source}

Mark King / May 19, 1995

\section{Samples collected}

- 24 identical samples from a near source piezometer (3-0-7); sample are indicated by "NS"

- 24 identical samples from a far source piezometer (11-0-6); samples are indicated by "FS"

- NS and FS sets have been split into 8 groups each; each group is composed of triplicate samples, for example the first set is NS-1(1), NS-1(2), NS-1(3)

\section{Errect of Simple Storne Time}

- Samples NS and FS I through 4 will be extracted in sequence, at regular intervals over the extraction period:

\begin{tabular}{|l|l|l|}
\hline Samples & Extraction Date & Analysis Date \\
\hline $\begin{array}{l}\text { NS-1(1), NS-1(2), NS-1(3) } \\
\text { FS-1(1), FS-1(2), FS-1(3) }\end{array}$ & May 19 & $\begin{array}{l}\text { all 24 samples at same time } \\
\text { (around May 31) }\end{array}$ \\
\hline $\begin{array}{l}\text { NS-2(1), NS-2(2), NS-3(3) } \\
\text { FS-2(1), FS-2(2), FS-2(3) }\end{array}$ & May 23 & \\
\hline $\begin{array}{l}\text { NS-3(1), NS-3(2), NS-3(3) } \\
\text { FS-3(1), FS-3(2), FS-3(3) }\end{array}$ & May 26 & \\
\hline $\begin{array}{l}\text { NS-4(1), NS-4(2), NS-4(3) } \\
\text { FS-4(1), FS-4(2), FS-4(3) }\end{array}$ & at end of extraction period & \\
\hline
\end{tabular}

keep a record of exact extraction and analysis dates

\section{Efrect of Extract Storage Time}

- Samples NS and FS 5 through 8 will be extracted at the same time and analyzed in sequence, at regular intervals over the analytical period:

\begin{tabular}{|l|l|l|}
\hline Samples & Extraction Date & Analysis Date \\
\hline $\begin{array}{l}\text { NS-5(1), NS-5(2), NS-5(3) } \\
\text { FS-5(1), FS-5(2), FS-5(3) }\end{array}$ & $\begin{array}{l}\text { all 24 samples at same time } \\
\text { (around May 19 or ASAP) }\end{array}$ & ASAP after extraction \\
\hline $\begin{array}{l}\text { NS-6(1), NS-6(2), NS-6(3) } \\
\text { FS-6(1), FS-6(2), FS-6(3) }\end{array}$ & & June 30 \\
\hline $\begin{array}{l}\text { NS-7(1), NS-7(2), NS-7(3) } \\
\text { FS-7(1), FS-7(2), FS-7(3) }\end{array}$ & & July 31 \\
\hline $\begin{array}{l}\text { NS-8(1), NS-8(2), NS-8(3) } \\
\text { FS-8(1), FS-8(2), FS-8(3) }\end{array}$ & & $\begin{array}{l}\text { at end of analytical period } \\
\text { (around end of August) }\end{array}$ \\
\hline
\end{tabular}

- keep a record of exact extraction and analysis dates 
Table 1. Summary of results from experirment to test the efifect of sample and extract storage time: results are averages of triplicate samples and units are in ug $\mathrm{L}$.

\begin{tabular}{|c|c|c|c|c|c|c|}
\hline & \multicolumn{6}{|c|}{ Extract storage } \\
\hline & \multicolumn{3}{|c|}{ near source } & \multicolumn{3}{|c|}{ far source } \\
\hline & 0 davs & 49 days & 69 days & 0 days & 49 davs & 69 davs \\
\hline $\begin{array}{l}\text { m-xylene } \\
\text { naphthalene }\end{array}$ & $\begin{array}{r}0 \\
1445\end{array}$ & $\begin{array}{r}0 \\
1585\end{array}$ & $\begin{array}{r}0 \\
1285\end{array}$ & $\begin{array}{r}568 \\
16806\end{array}$ & $\begin{array}{r}542 \\
16405\end{array}$ & $\begin{array}{r}587 \\
15148\end{array}$ \\
\hline indole+2-m.naph & 163 & 227 & 176 & 1397 & 1352 & 1338 \\
\hline 1-m.naph & 100 & 204 & 205 & 814 & 801 & 916 \\
\hline acenaphthene & 87 & 100 & 96 & 48 & 46 & 53 \\
\hline dibenzofuran & o] & $\underline{0}$ & 0 & 293 & 300 & 413 \\
\hline
\end{tabular}

\begin{tabular}{|c|c|c|c|c|c|c|c|c|}
\hline & \multicolumn{8}{|c|}{ Sample storage } \\
\hline & \multicolumn{4}{|c|}{ near source } & \multicolumn{4}{|c|}{ Er source } \\
\hline & 0 days & 5 days & 7 days & 10 divs & 0 days & 5 days & 7 days & 10 days \\
\hline m-xylene & & & $\begin{array}{r}0 \\
070\end{array}$ & 0 & 578 & $\begin{array}{r}636 \\
15010\end{array}$ & $\begin{array}{r}549 \\
16267\end{array}$ & 617 \\
\hline naphthalene & $\begin{array}{r}1396 \\
196\end{array}$ & $\begin{array}{r}1138 \\
115\end{array}$ & $\begin{array}{r}979 \\
8 ?\end{array}$ & $\begin{array}{r}1041 \\
87\end{array}$ & $\begin{array}{r}15209 \\
1375\end{array}$ & $\begin{array}{r}15910 \\
1421\end{array}$ & $\begin{array}{r}16367 \\
1326\end{array}$ & 16447 \\
\hline 1-m.naph & $\begin{array}{l}120 \\
190\end{array}$ & $\begin{array}{l}113 \\
136\end{array}$ & $\begin{array}{l}02 \\
72\end{array}$ & $\begin{array}{l}87 \\
97\end{array}$ & 807 & $\begin{array}{r}1421 \\
821\end{array}$ & $\begin{array}{r}1320 \\
834\end{array}$ & 837 \\
\hline acenaphthene & 96 & 96 & 96 & 102 & 47 & so & 52 & 61 \\
\hline dibenzofuran & 0 & 0 & I & 0 & 369 & 387 & 405 & 404 \\
\hline
\end{tabular}



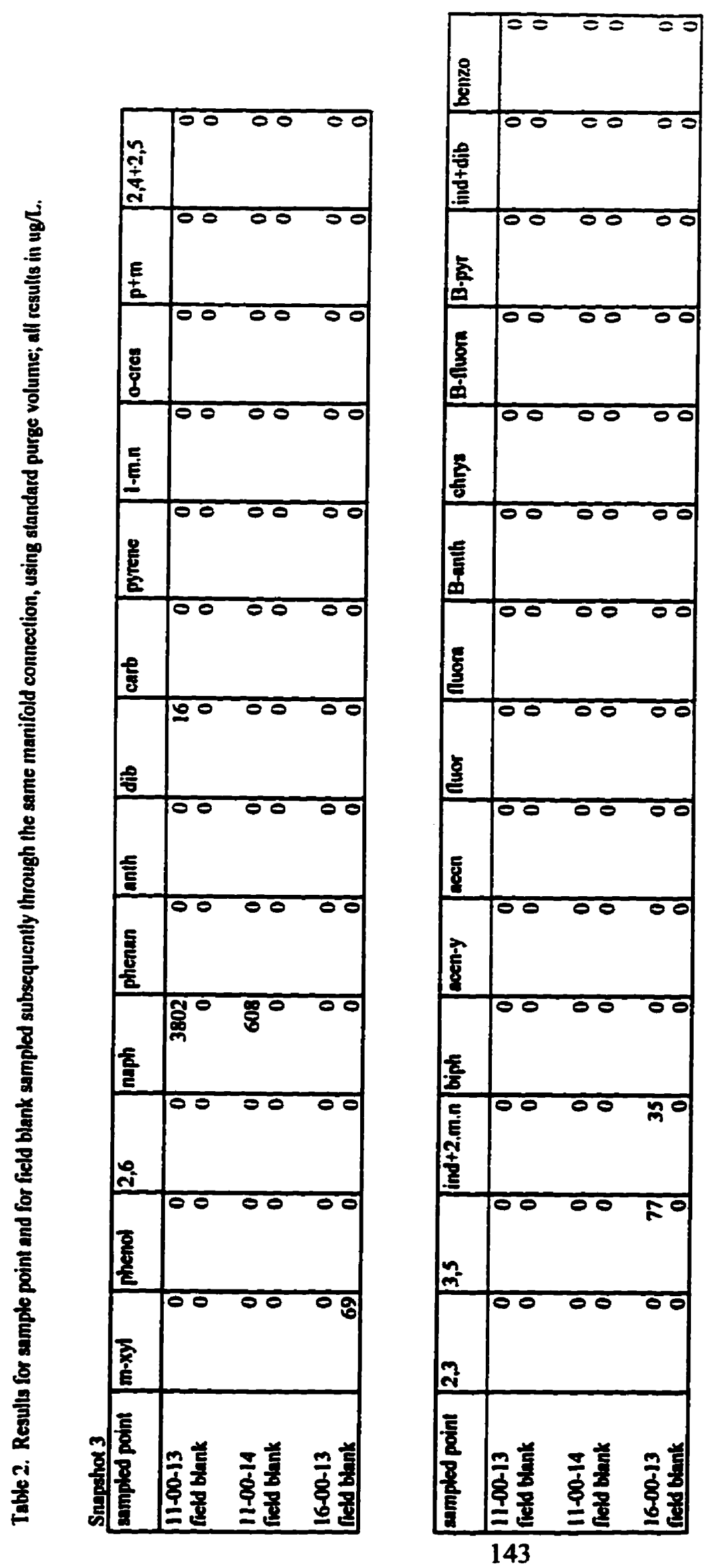


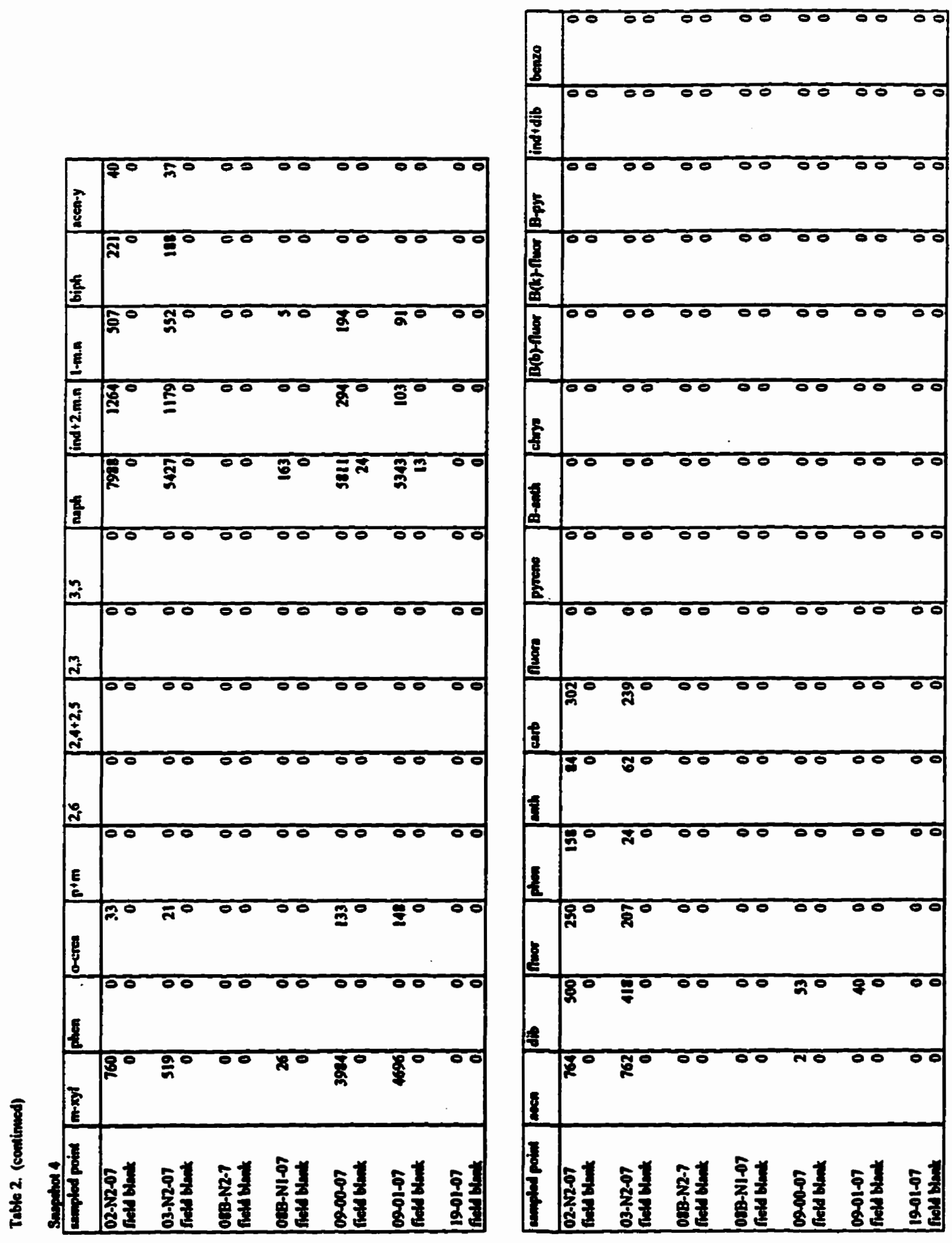




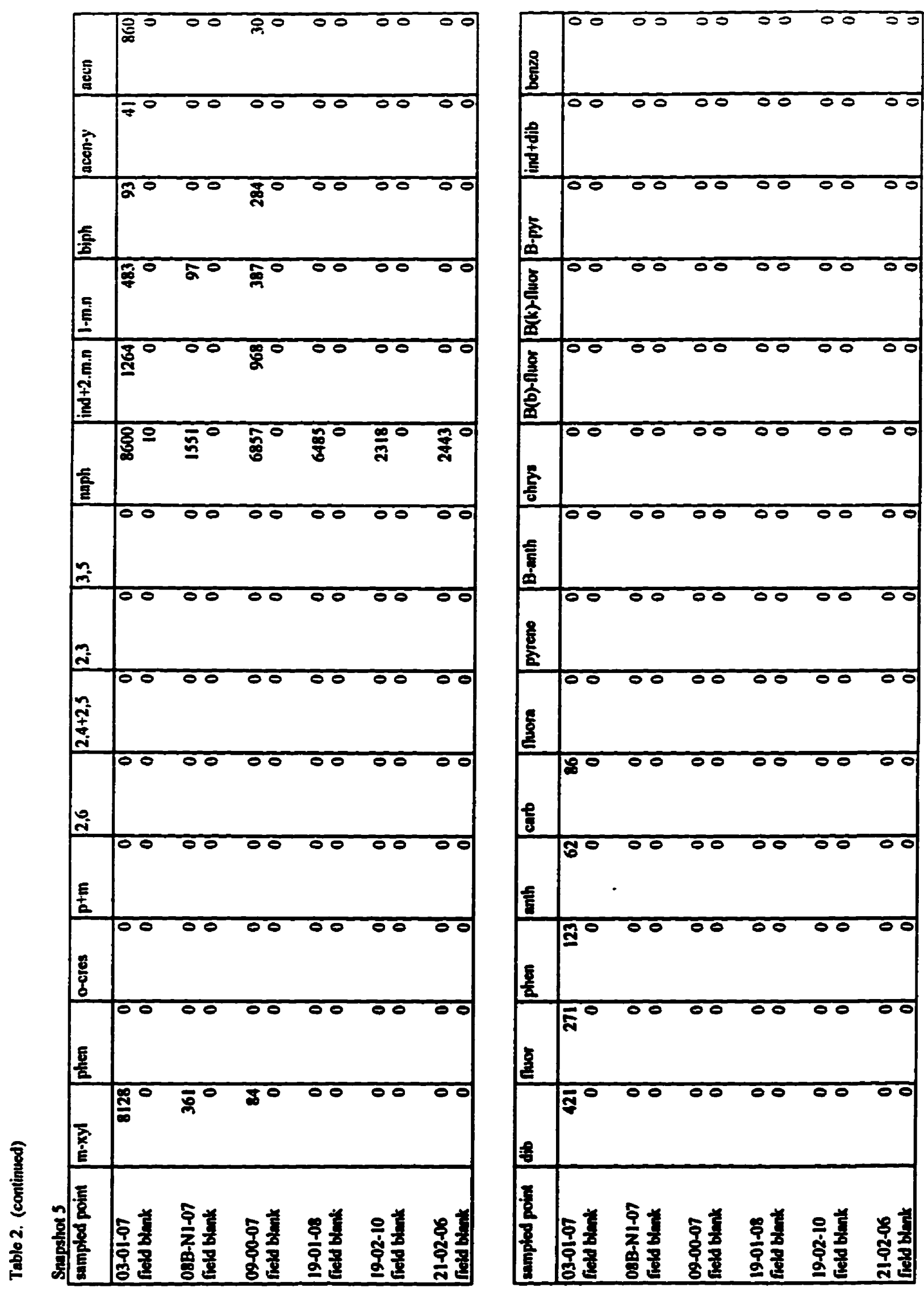




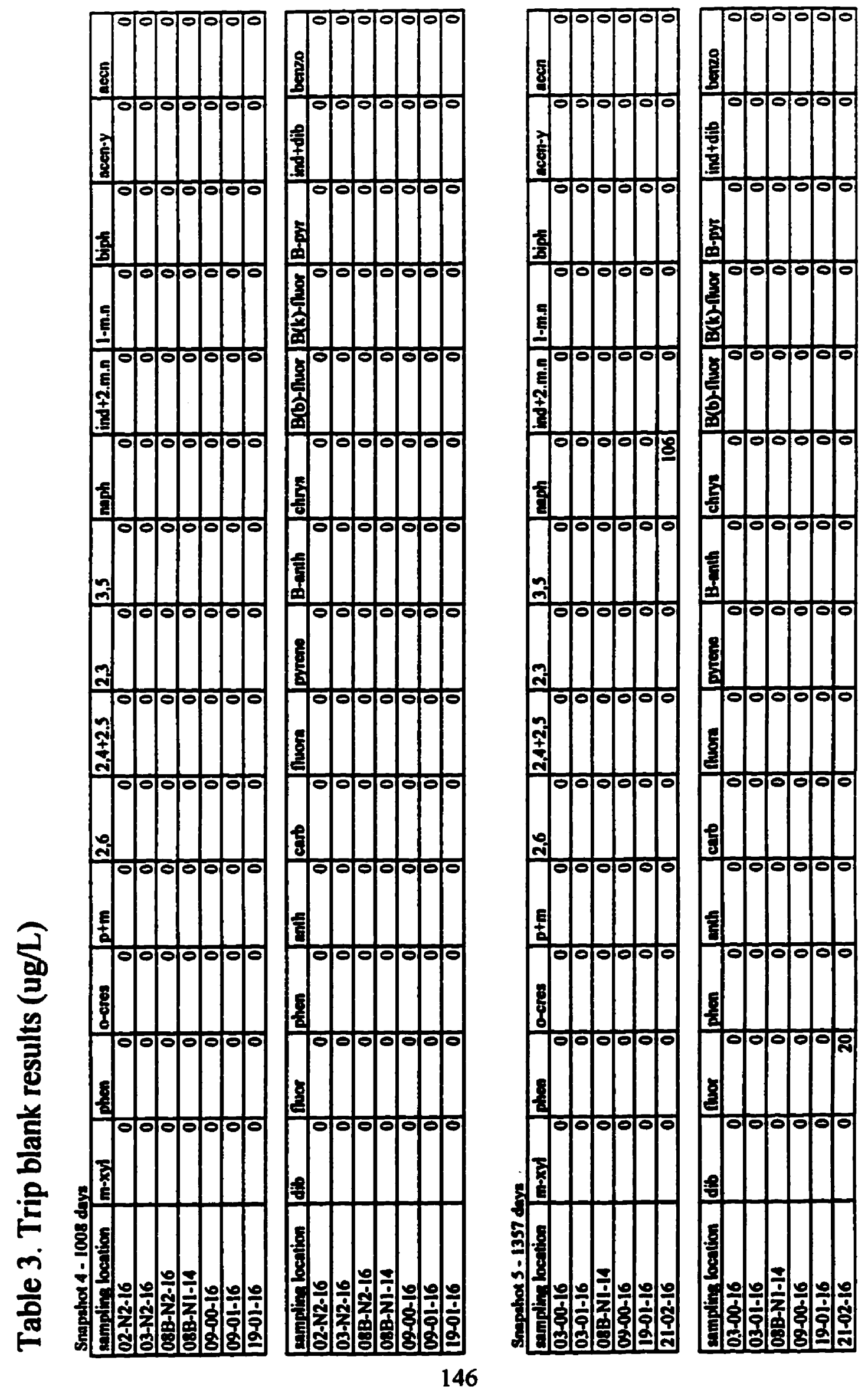


Table 4. Results of samples collected to evaluate whether sorption to teflon piezometer tubing has a significant effect on sample results, bulk sample was recovered from a stainless steel piezometer, then aliquots were either poured direetly into sample bottles or pumped into botules through $4 \mathrm{~m}$ of teflon tubing, pumped samples were preceded by a $50 \mathrm{mI}$ purge,similar to standard field sampling protocol.

\begin{tabular}{|c|c|c|c|c|c|c|c|c|}
\hline \multicolumn{9}{|c|}{ Near-source sampler (SS19-5) } \\
\hline & & Poure & & & & ?umped & & \\
\hline 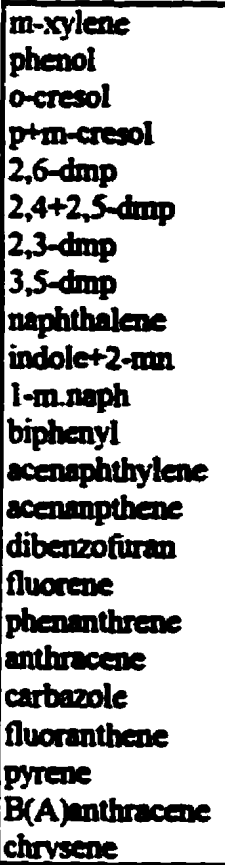 & $\begin{array}{r}0 \\
0 \\
0 \\
0 \\
0 \\
0 \\
0 \\
0 \\
2.42 \\
0.49 \\
0.32 \\
0.19 \\
0.03 \\
0.57 \\
0.24 \\
0.22 \\
0 \\
0.04 \\
0.17 \\
0 \\
0 \\
0 \\
0\end{array}$ & $\begin{array}{r}0 \\
0 \\
0 \\
0 \\
0 \\
0 \\
0 \\
0 \\
2.37 \\
0.48 \\
0.32 \\
0.18 \\
0.03 \\
0.57 \\
0.25 \\
0.22 \\
0 \\
0.04 \\
0.16 \\
0 \\
0 \\
0 \\
0\end{array}$ & $\begin{array}{r}0 \\
0 \\
0 \\
0 \\
0 \\
0 \\
0 \\
0 \\
2.42 \\
0.49 \\
0.32 \\
0.19 \\
0.03 \\
0.57 \\
0.25 \\
0.22 \\
0 \\
0.04 \\
0.18 \\
0 \\
0 \\
0 \\
0\end{array}$ & $\begin{array}{r}0 \\
0 \\
0 \\
0 \\
0 \\
0 \\
0 \\
0 \\
2.4 \\
0.48 \\
0.32 \\
0.19 \\
0.03 \\
0.58 \\
0.24 \\
0.22 \\
0 \\
0.04 \\
0.18 \\
0 \\
0 \\
0 \\
0\end{array}$ & $\begin{array}{r}0 \\
0 \\
0 \\
0 \\
0 \\
0 \\
0 \\
0 \\
2.4 \\
0.49 \\
0.32 \\
0.19 \\
0.03 \\
0.58 \\
0.24 \\
0.21 \\
0 \\
0.03 \\
0.16 \\
0 \\
0 \\
0 \\
0\end{array}$ & $\begin{array}{r}0 \\
0 \\
0 \\
0 \\
0 \\
0 \\
0 \\
0 \\
2.41 \\
0.47 \\
0.32 \\
0.18 \\
0.03 \\
0.57 \\
0.23 \\
0.2 \\
0 \\
0.04 \\
0.17 \\
0 \\
0 \\
0 \\
0\end{array}$ & $\begin{array}{r}0 \\
0 \\
0 \\
0 \\
0 \\
0 \\
0 \\
0 \\
2.48 \\
0.48 \\
0.33 \\
0.19 \\
0.03 \\
0.57 \\
0.25 \\
0.2 \\
0 \\
0.04 \\
0.15 \\
0 \\
0 \\
0 \\
0\end{array}$ & $\begin{array}{r}0 \\
0 \\
0 \\
0 \\
0 \\
0 \\
0 \\
0 \\
2.41 \\
0.48 \\
0.32 \\
0.18 \\
0.03 \\
0.57 \\
0.24 \\
0.21 \\
0 \\
0.04 \\
0.15 \\
0 \\
0 \\
0 \\
0\end{array}$ \\
\hline
\end{tabular}

Ferr-ecource sampler (SS34-5)

\begin{tabular}{|c|c|c|c|c|c|c|c|c|}
\hline & & Pourt & & & & imper & & \\
\hline 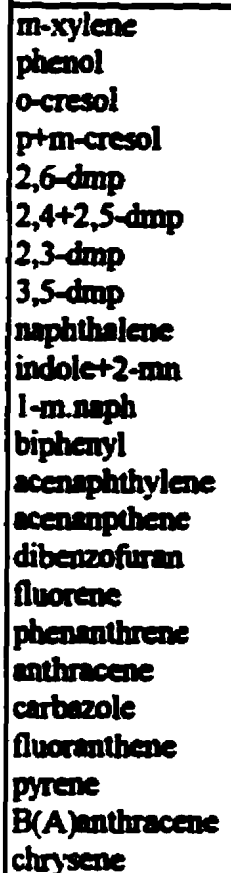 & $\begin{array}{r}0.66 \\
0 \\
0 \\
0 \\
0 \\
0 \\
0 \\
0 \\
6.5 \\
0 \\
0 \\
0 \\
0 \\
0 \\
0.06 \\
0 \\
0 \\
0 \\
0 \\
0 \\
0 \\
0 \\
0\end{array}$ & $\begin{array}{r}0.65 \\
0 \\
0 \\
0 \\
0 \\
0 \\
0 \\
0 \\
6.48 \\
0 \\
0 \\
0 \\
0 \\
0 \\
0.06 \\
0 \\
0 \\
0 \\
0 \\
0 \\
0 \\
0 \\
0\end{array}$ & $\begin{array}{r}0.67 \\
0 \\
0 \\
0 \\
0 \\
0 \\
0 \\
0 \\
6.62 \\
0 \\
0 \\
0 \\
0 \\
0 \\
0.06 \\
0 \\
0 \\
0 \\
0 \\
0 \\
0 \\
0 \\
0\end{array}$ & $\begin{array}{r}0.66 \\
0 \\
0 \\
0 \\
0 \\
0 \\
0 \\
0 \\
6.51 \\
0 \\
0 \\
0 \\
0 \\
0 \\
0.06 \\
0 \\
0 \\
0 \\
0 \\
0 \\
0 \\
0 \\
0\end{array}$ & $\begin{array}{r}0.65 \\
0 \\
0 \\
0 \\
0 \\
0 \\
0 \\
0 \\
6.51 \\
0.02 \\
0 \\
0 \\
0 \\
0 \\
0.06 \\
0 \\
0 \\
0 \\
0 \\
0 \\
0 \\
0 \\
0\end{array}$ & $\begin{array}{r}0.65 \\
0 \\
0 \\
0 \\
0 \\
0 \\
0 \\
0 \\
6.52 \\
0.02 \\
0 \\
0 \\
0 \\
0 \\
0.06 \\
0 \\
0 \\
0 \\
0 \\
0 \\
0 \\
0 \\
0 \\
\end{array}$ & $\begin{array}{r}0.68 \\
0 \\
0 \\
0 \\
0 \\
0 \\
0 \\
0 \\
6.56 \\
0 \\
0 \\
0 \\
0 \\
0 \\
0.06 \\
0 \\
0 \\
0 \\
0 \\
0 \\
0 \\
0 \\
0\end{array}$ & $\begin{array}{r}0.66 \\
0 \\
0 \\
0 \\
0 \\
0 \\
0 \\
0 \\
6.54 \\
0 \\
0 \\
0 \\
0 \\
0 \\
0.06 \\
0 \\
0 \\
0 \\
0 \\
0 \\
0 \\
0 \\
0\end{array}$ \\
\hline
\end{tabular}




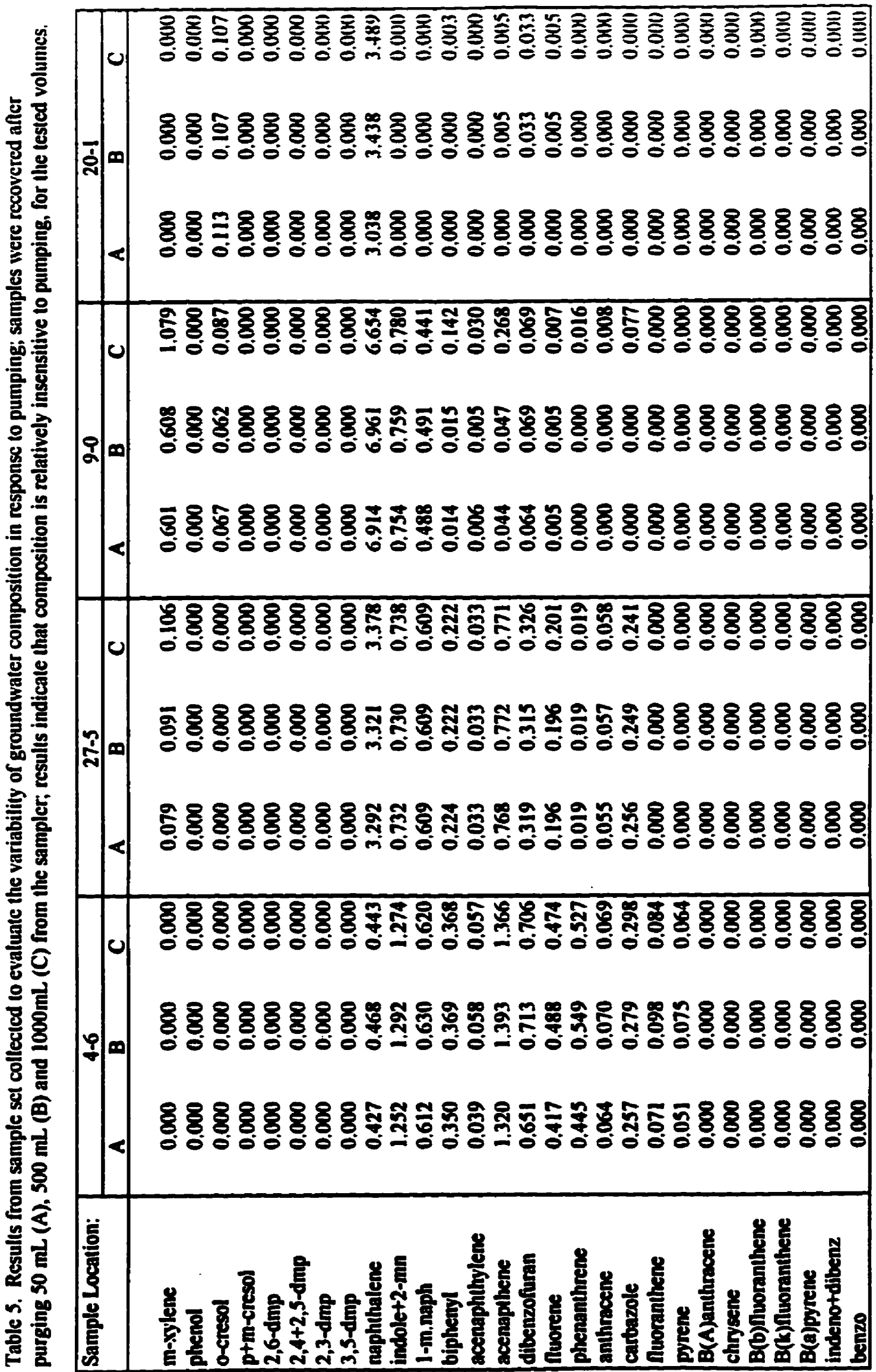




\section{APPENDIX 8}

\section{PROCEDURE FOR ESTIMATION OF PARTITIONING COEFFICIENTS}

\section{PURPOSE}

To estimate $K_{d} s$ for moderately hydrophobic creosote compounds.

\section{METHODS}

1.0 Preparation of stock solution

- add $1.2 \mathrm{ml}$ carbazole and $8.6 \mathrm{ml} \mathrm{m}$-xylene to $180 \mathrm{~mL}$ raw creosote (liquid only); mix well; split mixture into $2100 \mathrm{~mL}$ glass bottles; seal each with no headspace and place in freezer for several hours to increase viscosity

- $\quad$ pour one bottle of creosote mixture into a IL amber glass bottle,

- fill remainder of IL bottle with Borden groundwater taking care not to put blobs of creosote in suspension, seal with no headspace

- $\quad$ store at room temperature for three days (based on Malcolmson (1992) results re time to reach equilibrium)

- siphon $0.8 \mathrm{~L}$ of water from the creosote/water bottle into another glass bottle

\subsection{Determination of soil/water partitioning coefifients}

\subsection{Dilution of stock solution}

- prepare $\mathbf{4 0 0} \mathrm{ml}$ of each of the following by adding Borden groundwater: straight stock solution, $2 \mathrm{X}$ dilution, $4 \mathrm{X}$ dilution, $8 \mathrm{X}$ dilution, $16 \mathrm{X}$ dilution

- collect a $20 \mathrm{~mL}$ sample of each for analysis of initial concentration;

\subsection{Batch preparation}

- to a $100 \mathrm{~mL}$ (nominal) glass bottle add approximately $25 \mathrm{~g}$ Borden sand; fill remainder of bottle with solution (average solution volume $=103 \mathrm{~mL}$ ); seal with a crimped cap (silicon seal/metal cap)

- make up triplicate bottles for each solution for a total of 15 bottles (5 solutions X 3)

- make up a triplicate set of control bottles for each dilution by completely filling $100 \mathrm{~mL}$ glass bottles with solution (no sand), cap as above (5 solutions X 3)

- total "partitioning coefficient" bottles $=30$ bottles

\subsection{Batch treatment}

- agitate bottles gently at room temperature for 12 hours

- centrifuge the bottles for 15 minutes to settle the sediment

- collect $20 \mathrm{Ll}$ water sample from each bottle through the silicon cap with a syringe

- total "partitioning coeficient" samples $=5$ initial +30 batch $=35$ 
Table 1. Summary of results for batch determination of solids partitioning coefficients (Kd); stock solution results for the latter three compounds have not been incorporporated since they are considerably greater than plume concentrations.

\begin{tabular}{|c|c|c|c|c|c|c|}
\hline & & $\begin{array}{c}\text { Srock } \\
\text { Solution }\end{array}$ & 2xDihution & 4.3Dilution & 8XDilution & 16xDilution \\
\hline naphthalene & 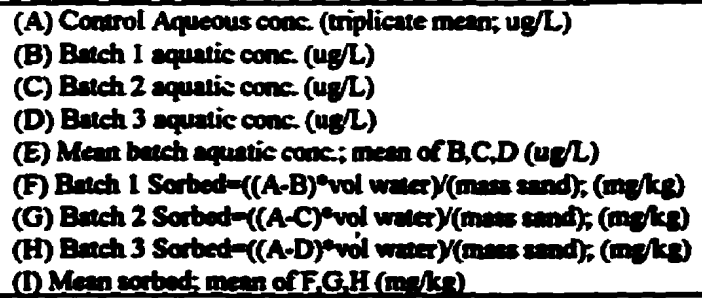 & $\begin{array}{l}9037 \\
8772 \\
8345 \\
8644 \\
8587 \\
1092 \\
2849 \\
1620 \\
1854 \\
\end{array}$ & \begin{tabular}{r|}
4449 \\
4072 \\
4220 \\
4158 \\
4150 \\
1553 \\
944 \\
1198 \\
1233 \\
\end{tabular} & \begin{tabular}{r|}
2129 \\
2083 \\
2071 \\
2075 \\
2076 \\
192 \\
239 \\
223 \\
218 \\
\end{tabular} & \begin{tabular}{r|}
1094 \\
1062 \\
1055 \\
1037 \\
1051 \\
131 \\
163 \\
236 \\
177 \\
\end{tabular} & \begin{tabular}{r|}
513 \\
506 \\
488 \\
489 \\
494 \\
25 \\
102 \\
98 \\
75 \\
\end{tabular} \\
\hline phemandireme & 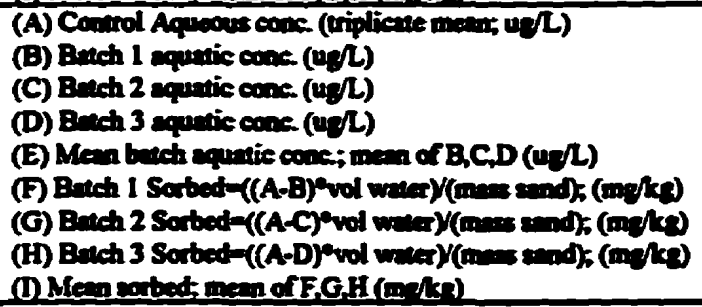 & \begin{tabular}{l|}
369 \\
277 \\
231 \\
271 \\
260 \\
462 \\
654 \\
487 \\
534
\end{tabular} & \begin{tabular}{l|}
282 \\
211 \\
209 \\
209 \\
310 \\
290 \\
301 \\
300
\end{tabular} & \begin{tabular}{r|}
97 \\
75 \\
69 \\
63 \\
69 \\
90 \\
117 \\
140 \\
116 \\
\end{tabular} & $\begin{array}{l}49 \\
36 \\
33 \\
38 \\
36 \\
52 \\
65 \\
46 \\
54\end{array}$ & \begin{tabular}{l|}
24 \\
19 \\
19 \\
18 \\
18 \\
23 \\
24 \\
29 \\
25
\end{tabular} \\
\hline dibenzofuren & 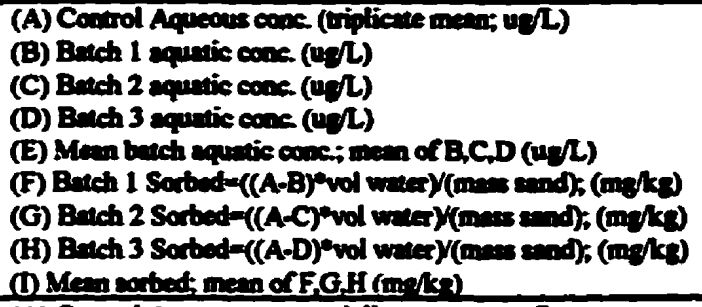 & & \begin{tabular}{l|}
735 \\
634 \\
637 \\
648 \\
646 \\
416 \\
322 \\
360 \\
366 \\
\end{tabular} & \begin{tabular}{l|}
349 \\
209 \\
313 \\
316 \\
195 \\
576 \\
148 \\
135 \\
286 \\
\end{tabular} & $\begin{array}{r}175 \\
82 \\
159 \\
157 \\
132 \\
384 \\
68 \\
76 \\
176 \\
\end{array}$ & \begin{tabular}{r|}
78 \\
49 \\
69 \\
74 \\
64 \\
119 \\
35 \\
16 \\
57 \\
\end{tabular} \\
\hline carbazole & 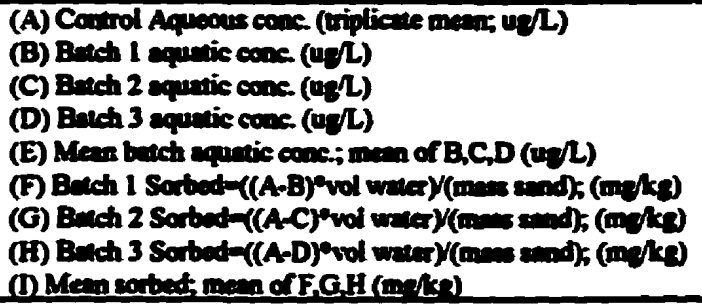 & & \begin{tabular}{l|}
384 \\
306 \\
316 \\
311 \\
311 \\
319 \\
220 \\
300 \\
300 \\
\end{tabular} & \begin{tabular}{r|}
178 \\
148 \\
175 \\
150 \\
158 \\
124 \\
10 \\
113 \\
82
\end{tabular} & $\begin{array}{r}104 \\
90 \\
89 \\
108 \\
96 \\
59 \\
62 \\
-16 \\
35 \\
\end{array}$ & \begin{tabular}{r|}
50 \\
45 \\
49 \\
47 \\
47 \\
21 \\
2 \\
12 \\
12 \\
\end{tabular} \\
\hline l-moph & 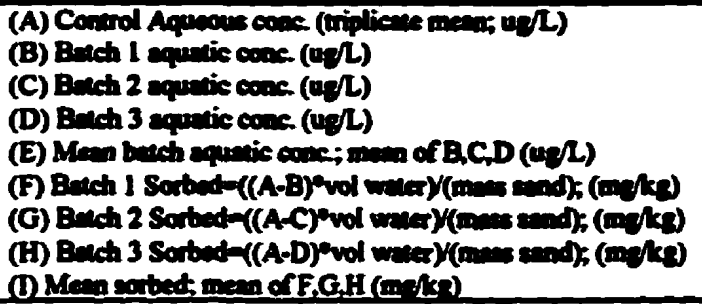 & & \begin{tabular}{r|r}
643 & 614 \\
619 & \\
604 & \\
612 & \\
122 & \\
98 & 163 \\
128 &
\end{tabular} & $\begin{array}{r}294 \\
253 \\
281 \\
283 \\
273 \\
170 \\
53 \\
46 \\
90\end{array}$ & $\begin{array}{r}109 \\
107 \\
100 \\
98 \\
102 \\
7 \\
34 \\
44 \\
28 \\
\end{array}$ & $\begin{array}{r}55 \\
54 \\
44 \\
43 \\
47 \\
3 \\
46 \\
48 \\
32 \\
\end{array}$ \\
\hline
\end{tabular}


Table 2. Summary of Kd estimates

\begin{tabular}{|c|c|c|c|c|c|c|c|c|}
\hline naphthalene & \multicolumn{2}{|c|}{ phenanthrene } & \multicolumn{2}{|c|}{ dibenzofuran } & \multicolumn{2}{|c|}{ carbazole } & \multicolumn{2}{|c|}{ 1-m.naphthalene } \\
\hline $\begin{array}{l}\text { sorbed } \\
\text { conc. } \\
\text { (mg/kg }\end{array}$ & $\begin{array}{l}\text { aqueous } \\
\text { conc. } \\
\text { (ug/L) }\end{array}$ & $\begin{array}{l}\text { sorbed } \\
\text { conc. } \\
\text { (mg/kg }\end{array}$ & $\begin{array}{l}\text { aqueous } \\
\text { conc. } \\
\text { (ug/L) }\end{array}$ & $\begin{array}{l}\text { sorbed } \\
\text { conc. } \\
\text { (mg/kg }\end{array}$ & $\begin{array}{l}\text { aqueous } \\
\text { conc. } \\
\text { (ug/L) }\end{array}$ & $\begin{array}{l}\text { sorbed } \\
\text { conc. } \\
(\mathrm{mg} / \mathrm{kg}\end{array}$ & $\begin{array}{c}\text { aqueous } \\
\text { conc. } \\
(\mathrm{ug} / \mathrm{L})\end{array}$ & $\begin{array}{l}\text { sorbed } \\
\text { conc. } \\
\text { (mg/kg }\end{array}$ \\
\hline 1854 & 260 & 534 & & & & & & \\
\hline 1232 & 209 & 300 & 646 & 366 & 311 & 300 & 612 & 128 \\
\hline 218 & 69 & 116 & 195 & 286 & 158 & 82 & 273 & 90 \\
\hline 177 & 36 & 54 & 132 & 176 & 96 & 35 & 102 & 28 \\
\hline 75 & 18 & 25 & 64 & 57 & 47 & 12 & 47 & 32 \\
\hline 0.22 & \multicolumn{2}{|c|}{1.80} & \multicolumn{2}{|c|}{0.67} & \multicolumn{2}{|c|}{0.83} & \multicolumn{2}{|c|}{0.24} \\
\hline 0.94 & \multicolumn{2}{|c|}{0.94} & \multicolumn{2}{|c|}{0.32} & \multicolumn{2}{|c|}{0.87} & \multicolumn{2}{|c|}{0.80} \\
\hline
\end{tabular}




\section{APPENDIX 9 \\ IN SITU $K_{d}$ ESTIMATION}

\section{BACKGROUND}

Samples of groundwater and sand were collected from within the plume to obtain an in situ $K_{d}$ estimate. Groundwater samples were collected from three different multi-level samplers in order to locate vertical zones where plume concentrations were relatively consistent between three to four vertically adjacemt sampling points. Groundwater results, shown in Table 1 , indicate detection of zones that were suitable with respect to several moderately hydrophobic compounds. Sand cores were subsequently obtained within these zones, and results are provided in Table 2. Table 3 provides results of the estimation technique, where average groundwater concentration has been related to estimated sorbed concentration. 


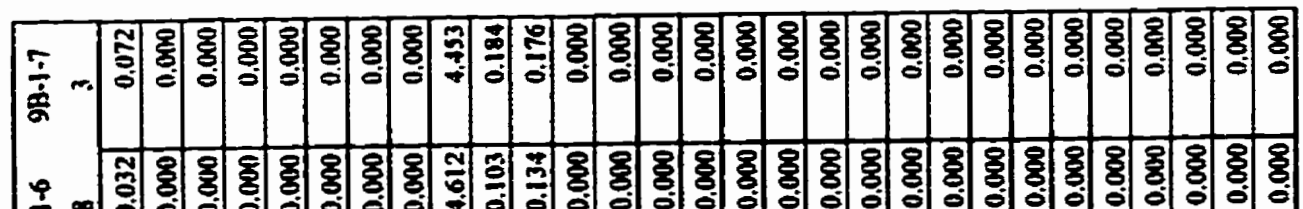

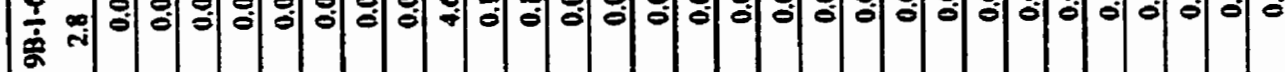

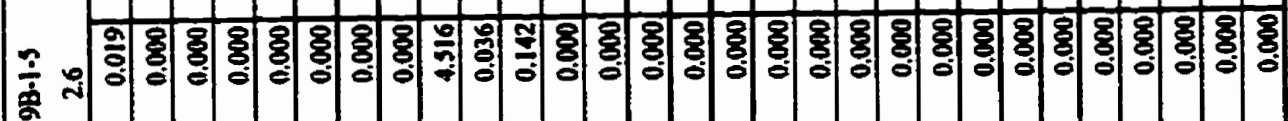

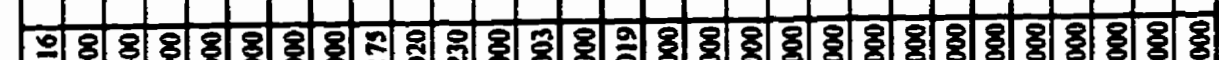
I - 항

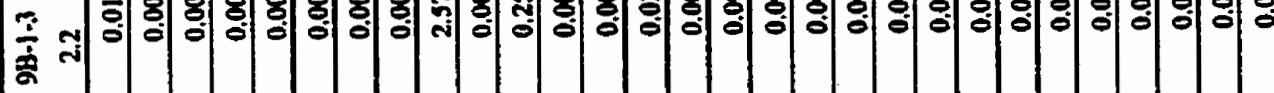

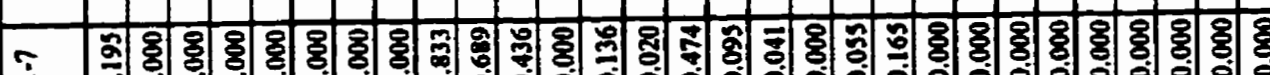

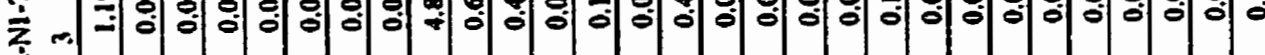

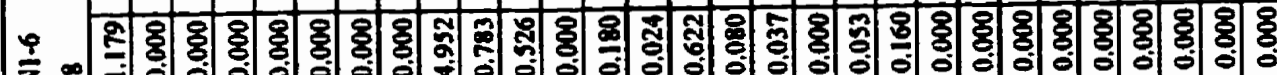

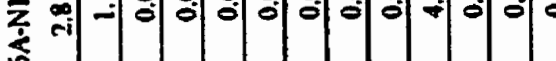

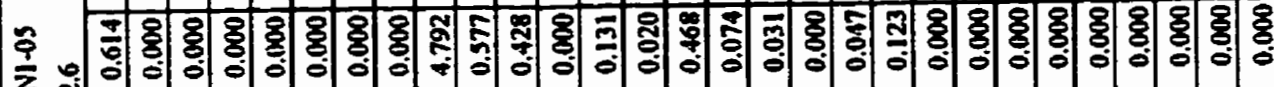
西

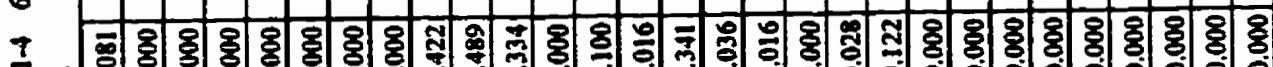

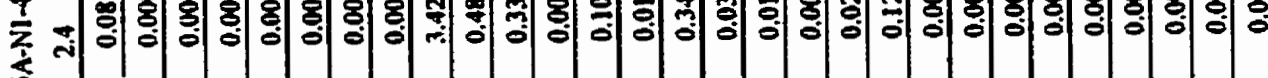

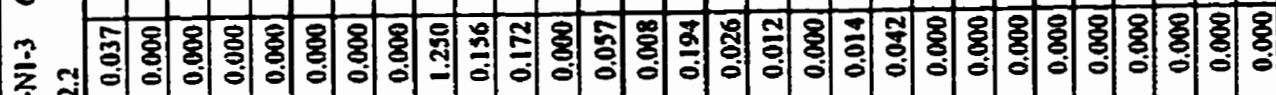
$\dot{8}$

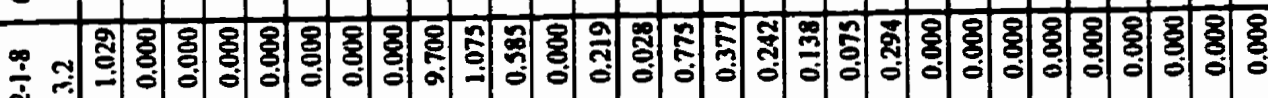

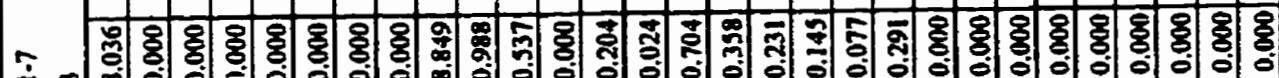
倞

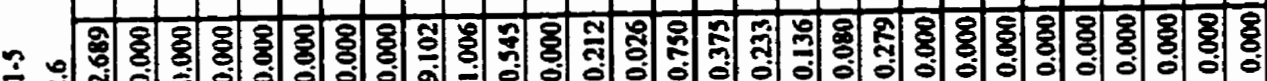
$\dot{\mathrm{i}}$ ने

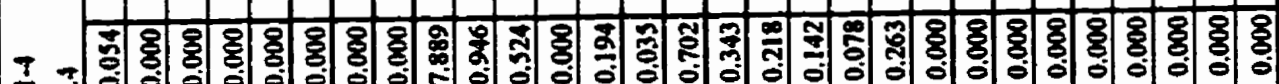

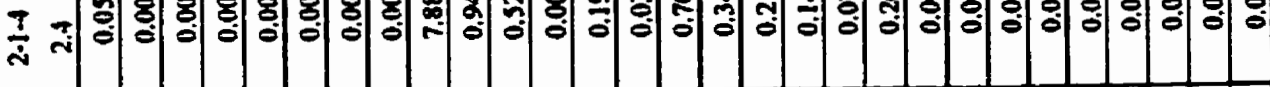


Table 2. Results for core samples collected adjacent to groundwater sampling sites; units are mg/kg wet weight and have not been corrected for compound mass present in incerstitial water.

\begin{tabular}{|c|c|c|c|}
\hline $\begin{array}{l}\text { location: } \\
\text { depth lop }(m) \text { : } \\
\text { depph bortom (m): }\end{array}$ & $\begin{array}{l}2-1 \\
2.74 \\
3.05 \\
\end{array}$ & $\begin{array}{c}6 \mathrm{~A}-\mathrm{NI} \\
2.44 \\
2.74 \\
\end{array}$ & $\begin{array}{l}9 \mathrm{~B}-1 \\
2.44 \\
2.74 \\
\end{array}$ \\
\hline m-sviene & 0.505 & 0.157 & 0.205 \\
\hline Phenol & 0.000 & 0.000 & 0.000 \\
\hline o-cresol & 0.000 & 0.000 & 0.000 \\
\hline$p+m$-ctesol & 0.000 & 0.000 & 0.000 \\
\hline 2.6-dimp & 0.005 & 0.004 & 0.002 \\
\hline $2,4+2.5-\mathrm{dinp}$ & 0.000 & 0.000 & 0.000 \\
\hline$\overline{2,3-\mathrm{mmp}}$ & 0.000 & 0.000 & 0.000 \\
\hline 3,5-dmp & 0.000 & 0.000 & 0.000 \\
\hline naphthalene & 2.001 & 0.830 & 1.204 \\
\hline indolet2-m.n. & 0.433 & 0.226 & 0.037 \\
\hline l-m.naph & 0.234 & 0.164 & 0.116 \\
\hline IS & 0.000 & 0.000 & 0.000 \\
\hline bjphenul & 0.245 & 0.134 & 0.000 \\
\hline acenaphithylen: & 0.023 & 0.014 & 0.002 \\
\hline acen aphithene & 0.573 & 0.291 & 0.004 \\
\hline dibenzofuran & 0.493 & 0.068 & 0.000 \\
\hline fluorene & 0.346 & 0.030 & 0.000 \\
\hline phenanthrene & 0.580 & 0.000 & 0.001 \\
\hline anthrecente & 0.085 & 0.030 & 0.000 \\
\hline carbazole & 0.315 & 0.101 & 0.000 \\
\hline fluoramthene & 0.000 & 0.000 & 0.000 \\
\hline prrene & 0.000 & 0.000 & 0.000 \\
\hline B(a)anthracence & 0.002 & 0.000 & 0.000 \\
\hline chrysene & 0.000 & 0.000 & 0.000 \\
\hline B(b)fuoram hene & 0.000 & 0.000 & 0.000 \\
\hline B(k)fuoranthene & 0.000 & 0.000 & 0.000 \\
\hline E(a)purreas & 0.000 & 0.000 & 0.000 \\
\hline indenotdibenz & 0.000 & 0.000 & 0.000 \\
\hline benzo & 0.000 & 0.000 & 0.000 \\
\hline Water content & 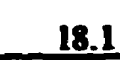 & 17.4 & 18. \\
\hline
\end{tabular}


Table 3. Summary of in situ Kd estimation; groundwater results were calculated using data from sampling points that "bracket" the cored interval (see Table 1); sorbed concentration was estimate by subtracting dissolved mass from the soil concentrations given in Table 2, on the basis of sampl water content (Table 2) and mean groundwater concentration (this table).

\begin{tabular}{|c|c|c|c|c|c|c|c|c|}
\hline \multirow[t]{2}{*}{ Compound } & \multirow[t]{2}{*}{$\begin{array}{l}\text { Sampling } \\
\text { location }\end{array}$} & \multirow{2}{*}{$\begin{array}{l}\text { Distance } \\
\text { from } \\
\text { source } \\
\text { (m) }\end{array}$} & \multicolumn{3}{|c|}{$\begin{array}{l}\text { Groundwater results } \\
\text { for cored interval }\end{array}$} & \multirow{2}{*}{$\begin{array}{c}\text { Sorbed } \\
\text { concentration } \\
\text { (ug/ks) }\end{array}$} & \multirow{2}{*}{$\begin{array}{c}\text { Point } \\
\text { Kd } \\
\text { eximate } \\
(\mathrm{cm} 3 / \mathrm{g})\end{array}$} & \multirow{2}{*}{$\begin{array}{c}\text { Average } \\
\text { Kd } \\
(\mathrm{cm} / \mathrm{g})\end{array}$} \\
\hline & & & $\begin{array}{l}\text { Mean } \\
\text { (ug/L) }\end{array}$ & $\mathbf{n}$ & $\begin{array}{l}\text { stendard } \\
\text { devintion }\end{array}$ & & & \\
\hline naphthalene & $\begin{array}{c}2-I \\
6 A-N 1 \\
9 B-1\end{array}$ & $\begin{array}{c}2.5 \\
8 \\
16.75\end{array}$ & $\begin{array}{l}8975 \\
4389 \\
4434\end{array}$ & $\begin{array}{l}4 \\
3 \\
3\end{array}$ & $\begin{array}{l}520 \\
686 \\
188 \\
\end{array}$ & $\begin{array}{l}739.05 \\
209.59 \\
606.61 \\
\end{array}$ & $\begin{array}{l}0.08 \\
0.05 \\
0.14 \\
\end{array}$ & 0.09 \\
\hline phenanthrene & $\begin{array}{c}2-1 \\
6 A-N 1 \\
9 B-1 \\
\end{array}$ & $\begin{array}{c}2.5 \\
8 \\
16.75 \\
\end{array}$ & $\begin{array}{l}140 \\
\mathrm{ND} \\
\mathrm{ND}\end{array}$ & $\begin{array}{l}4 \\
3 \\
3 \\
\end{array}$ & - & 659.81 & 4.70 & 4.70 \\
\hline dibenzofuran & $\begin{array}{c}2-1 \\
6 A-N 1 \\
9 B-1 \\
\end{array}$ & $\begin{array}{c}2.5 \\
8 \\
16.75 \\
\end{array}$ & \begin{tabular}{r|}
358 \\
63 \\
$\mathrm{ND}$ \\
\end{tabular} & $\begin{array}{l}4 \\
3 \\
3 \\
\end{array}$ & $\begin{array}{r}22 \\
19 \\
- \\
\end{array}$ & $\begin{array}{r}517.33 \\
68.85\end{array}$ & $\begin{array}{l}1.44 \\
1.08\end{array}$ & 1.26 \\
\hline carbazole & $\begin{array}{c}2-1 \\
6 A-N 1 \\
9 B-1\end{array}$ & $\begin{array}{c}2.5 \\
8 \\
16.75 \\
\end{array}$ & $\begin{array}{l}275 \\
135 \\
\text { ND }\end{array}$ & $\begin{array}{l}4 \\
3 \\
3\end{array}$ & $\begin{array}{l}22 \\
18\end{array}$ & $\begin{array}{r}322.55 \\
95.40\end{array}$ & $\begin{array}{l}1.17 \\
0.71\end{array}$ & 0.94 \\
\hline 1-m.naphthalen & $\begin{array}{c}2-1 \\
6 A-N 1 \\
9 B-1\end{array}$ & $\begin{array}{c}2.5 \\
8 \\
16.75\end{array}$ & $\begin{array}{l}537 \\
429 \\
169\end{array}$ & $\begin{array}{l}4 \\
3 \\
3\end{array}$ & $\begin{array}{l}37 \\
78 \\
44\end{array}$ & $\begin{array}{l}179.58 \\
117.23 \\
106.02\end{array}$ & \begin{tabular}{l|}
0.33 \\
0.27 \\
0.63
\end{tabular} & 0.41 \\
\hline
\end{tabular}




\section{APPENDIX 10 \\ MICROCOSM EXPERIMENT}

\section{BACKGROUND}

Experiment conducted by Mark King and Marianne VanderGriendt; set up on May 10, 1995; write-up by Marianne VanderGriendt and Mark King.

\section{MATERIALS AND METHODS:}

Core Material:

After collection, cores were stored at 4 degrees Celsius until use. The core used for this experiment was collected from Canada Forces Base Borden on Jan 25, 1995 (by Mark King). The top of the core was from a depth of $2.18 \mathrm{~m}$ and the bottom was labelled at an $3.35 \mathrm{~m}$ depth, with collection occurring $0.61 \mathrm{~m}$ down gradient of $3-\mathrm{N} 1$. Sections $0.3 \mathrm{~m}$ in length were cut and the inner diameter of each section was pared out of the core barrel. Care was taken to avoid material coming from the $1-2 \mathrm{~cm}$ at the ends, and around the sides of the core barrel. One hundred and forty grams $(140 \mathrm{~g})$ of mixed parings were weighted into each of the 6 autoclaved control microcosms and autoclaved for $1 \mathrm{hr}$. on 3 separate occassions over the course of a 5 day period (Days 1, 2, and 5). Inner diameter section of the core were mixed and stored at 4 degrees Celcius, in sterile $1 \mathrm{~L}$ mason jars, for 5 days until control autoclaving was complete. Again, $140 \mathrm{~g}$ of core material from the mason jars was allocated to the active microcosms immediately before set up.

\section{Groundwater}

Used Mark's collected groundwater from September of 1994-uncontaminated Borden groundwater.

Since this experiment was aerobic, no nitrogen purging of the groundwater occurred. The initial dissolved oxygen content of the water was not measured but was assummed to be aerobic, since the carboy was stored at 4 degrees Celcius with a sizable headspace for quite some time (ie. expected dissolved oxygen approximately $6-7 \mathrm{mg} / \mathrm{L}$ ).

\section{Aerobic microcosms}

All equipment used during the set up was sterilized prior to use and aseptic technique was empioyed throughout the experiment. Twelve $1100 \mathrm{ml}$ glass bottle microcosms, with 0-ring tap stopcocks (J. Young Scientific Glassware Ltd.), and mininert (R) capped side arms were used for the experiments, so that continuous re-sampling of the microcosms could be carried out, while maintaining an atmospheric seal. Of these 12 bottles, 6 consisted of control microcosms (core 
material previously sterilized). Of these 6 controis, 3 received $3.4 \mathrm{mls}$ of a $10 \%$ solution of sodium azide ((1) CONTROL-1, (2)CONTROL-2, (3)CONTROL-3). The remaining 3 control bottles did not receive sodium azide addition ((10)CONTROL-4, (11)CONTROL-5, (12)CONTROL-6) but the groundwater used in these microcosms was filter sterilized through a 0.2 um membrane filter (these controls were prepared for Jennifer Maud's specific needs). The remaining 6 bottles were active microcosms, with 3 unammended bottles ((4)ACTIVE-1, (5)ACTIVE-2, (6)ACTIVE-3) and 3 bottles ammended with an inorganic nutrient medium called modified Bushnell Haas medium ((7)ACTIVE-4+MBH, (8)ACTIVE-5+MBH, (9)ACTIVE-6+MBH).

All bottles were placed in the sterile air flow cabinet were sodium azide addition ( $3.4 \mathrm{mls}$ of a $10 \%$ solution of sodium azide) was made to each of the 3 appropriate controls ((1) CONTROL-1, (2)CONTROL-2, (3)CONTROL-3) and 6.9mls of the inorganic nutrient medium (modified Bushnell Haas medium) was added to each of the appropriate active microcosms ((7)ACTIVE4+MBH, (8)ACTIVE-5+MBH, (9)ACTIVE-6+MBH). The modified Bushnell Haas medium consisted of per Liter: K2HPO4, $1.0 \mathrm{~g}$; KH2PO4, $1.0 \mathrm{~g}$; NH4NO3, $1.0 \mathrm{~g} ; \mathrm{MgSO} \mathbf{N}^{\star} 7 \mathrm{H} 2 \mathrm{O}, 0.2 \mathrm{~g}$; Cacl2*2H2O, 0.02g; Fecl3, 0.005g; distilled H2O, 1000m/s; and pH to 7.0 (Modified from Mueller et. al. 1991. ES and T,25:1045-1055). All bottles received 380mis of diluted creosote contacted water (method of composition described below) via a graduated cylinder. For this experiment, filter sterilized (0.2um filter) Borden groundwater was used to prepare the creosote contacted water so that sterile controls, without azide could be prepared for Jennifer Maud. After dispensing, bottles were immediately closed, leaving a $660 \mathrm{ml}$ headspace within them. Microcosms were incubated in a 10 degree Celcius incubator under static conditions.

Note: A few of the necks of the large bottles broke after the initial set up and had to be transfered to a smaller sized $800 \mathrm{ml}$ bottle. This resulted in a smaller sized headspace within the microcosm. However, since oxygen content within the microcosm was measured at each sampling time, oxygen depletion would be detected if it occurred.

Bottle Transfers occured on:

May 10, 1995

(6)ACTIVE-3 AND (8)ACTIVE-5+MBH

May 15, 1995

(7)ACTIVE-4+MBH

\section{Microcosm Sampling}

Microcosms were removed from the incubator, and placed in the sterile air flow cabinet and the sampling procedure went as follows:

A $20 \mathrm{ml}$ ground glass syringe (rinsed $1 X$ with $2.5 \mathrm{mls}$ of methanol and $3 \mathrm{x}$ with milli $Q$ water) was fitted with a sterile disposable $22 \mathrm{~g}$ needle tip. The stopcock of the bottle was opened and the bottle was gently placed on its side to allow groundwater to fill the sidearm. The needle tip of the syringe was inserted into the sidearm of the bottle by unlocking the mininert valve and $13 \mathrm{mls}$ of 
groundwater was removed from the microcosm. The $13 \mathrm{ml}$ sample was immediately placed in an $18 \mathrm{ml}$ hypovial containing $3 \mathrm{~g}$ of sodium chloride and $0.3 \mathrm{mls}$ of $1 \mathrm{~N}$ hydrochloric acid (standard creosote sample preparation. This vial was crimped with a teflon septa and aluminum seal and transported to the organic geochemistry laboratory were Kim Hamilton added the $1 \mathrm{ml}$ of methylene chloride (with internal standard) and did the organic analysis for PAHs etc. After this sample was taken, another 3.5 to $4 \mathrm{mls}$ was collected for Jennifer Maud's biological assays.

After the sampling had occurred, the mininert valve was left open for a short time to allow sterile air from within the sterile air flow cabinet to replentish the vacuum within the bottle. The syringe used to sample the bottles was cleaned, as previously described, between bottles and new sterile syringes were also used.

The headspace of the bottles was sampled for carbon dioxide and oxygen by withdrawing a $5 \mathrm{ml}$ gas sample via a syringe, and injecting it onto a gas partitioner with a thermal conductivity detector (two columns in series - D and molecular sieve). After sampling for $\mathrm{CO} 2$ and $\mathrm{O} 2$ had occured, the tap of the bottle was closed and the bottles were placed back in the 10degree Celcius incubator. Sampling times occurred on the following dates:

May 11, 1995 - Set 1 - Day 1

May 15, 1995 - Set 2 - Day 5

May 18, 1995 - Set 3 - Day 8

May 25, 1995 - Set 4 - Day 15

June 8, 1995 - Set 5 - Day 29

Preparation of Creosote Contacted Water:

To prepare the creosote contacted groundwater, $150 \mathrm{mls}$ of pure creosote was placed into a $6 \mathrm{~L}$ erlenmyer flask and $6 \mathrm{~g}(6.95 \mathrm{mls})$ of $\mathrm{m}$-xylene and $0.95 \mathrm{~g}$ of carbazole were added in. Four liters of filter sterilized Borden groundwater was added along with a teflon stir bar and the flask was tightly capped with a teflon wrapped stopper. The flask was stirred for $\mathbf{4 8}$ hrs. and sampled to determine initial concentrations of PAHs, etc.

Preparation of Diluted Creosote Contacted Water:

Creosote contacted water was carefully siphoned off of the creosote and collected in a bottle with a teflon-faced septa. A 4.33L volume was made by adding $2.63 \mathrm{~L}$ of groundwater and $1.7 \mathrm{~L}$ of creosote contacted water. Diluted creosote contacted water was allowed to stir for $30 \mathrm{~min}$. before dispensing to the microcosms.

\section{CREOSOTE AND HEADSPACE RESULTS}

Results of creosote compound monitoring are provided in Table 1. Sample calibration tables for analysis of oxygen and carbon dioxide in microcosm headspace are shown as Tables 2 and 3, respectively, and a summary of headspace data is provided in Table 4. 
<smiles>[CH]1[CH]C1</smiles> 
Table 2. Standard table for calibration and analysis of oxygen in microcosm headspace; analysis for June 8, 1995 - Day 29 of experiment.

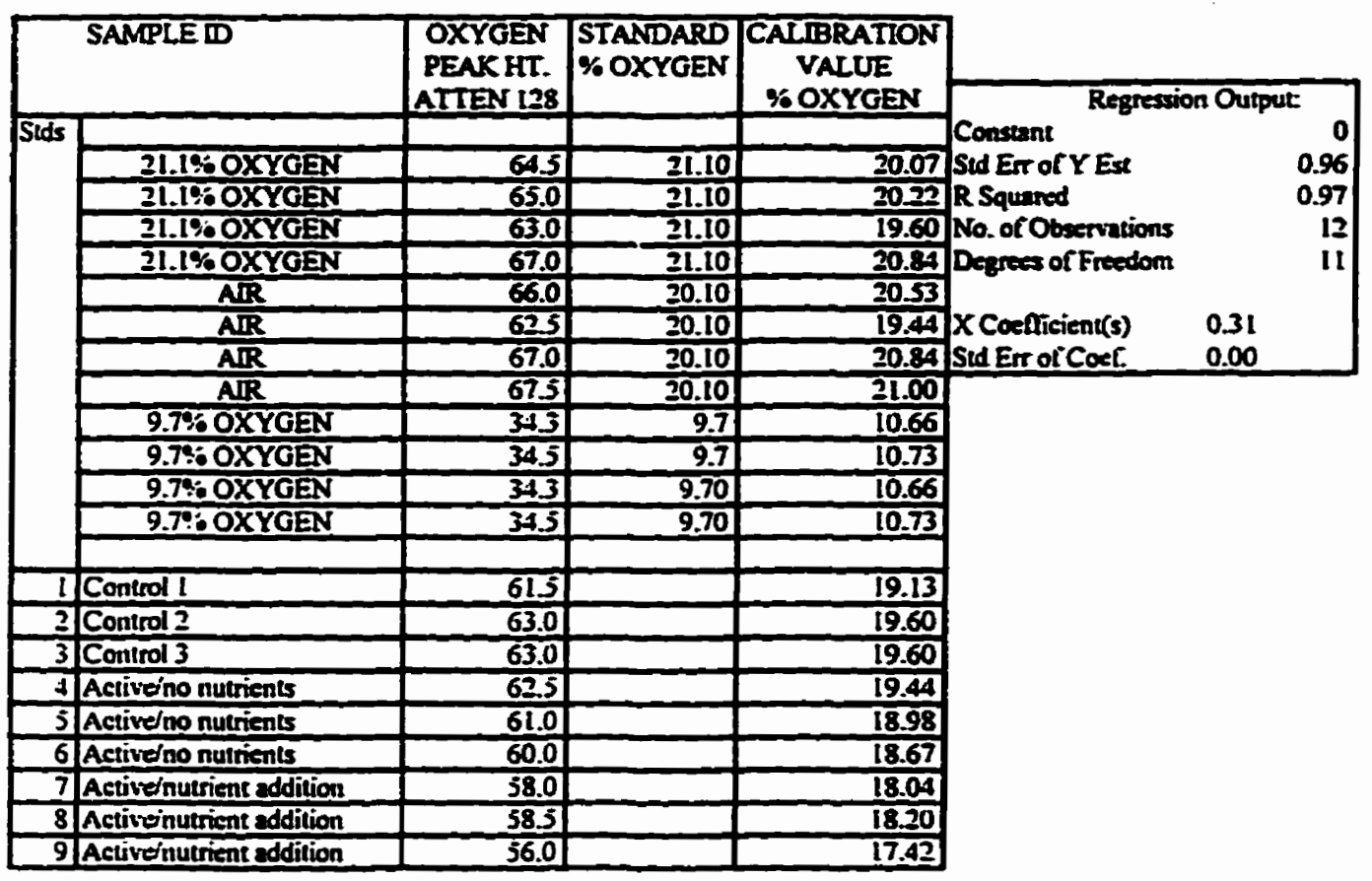

Table 3. Standard table for calibration and analysis of Carbon dioxide in microcosm headspace; analysis for June 8, 1995 - Day 29 of experinient.

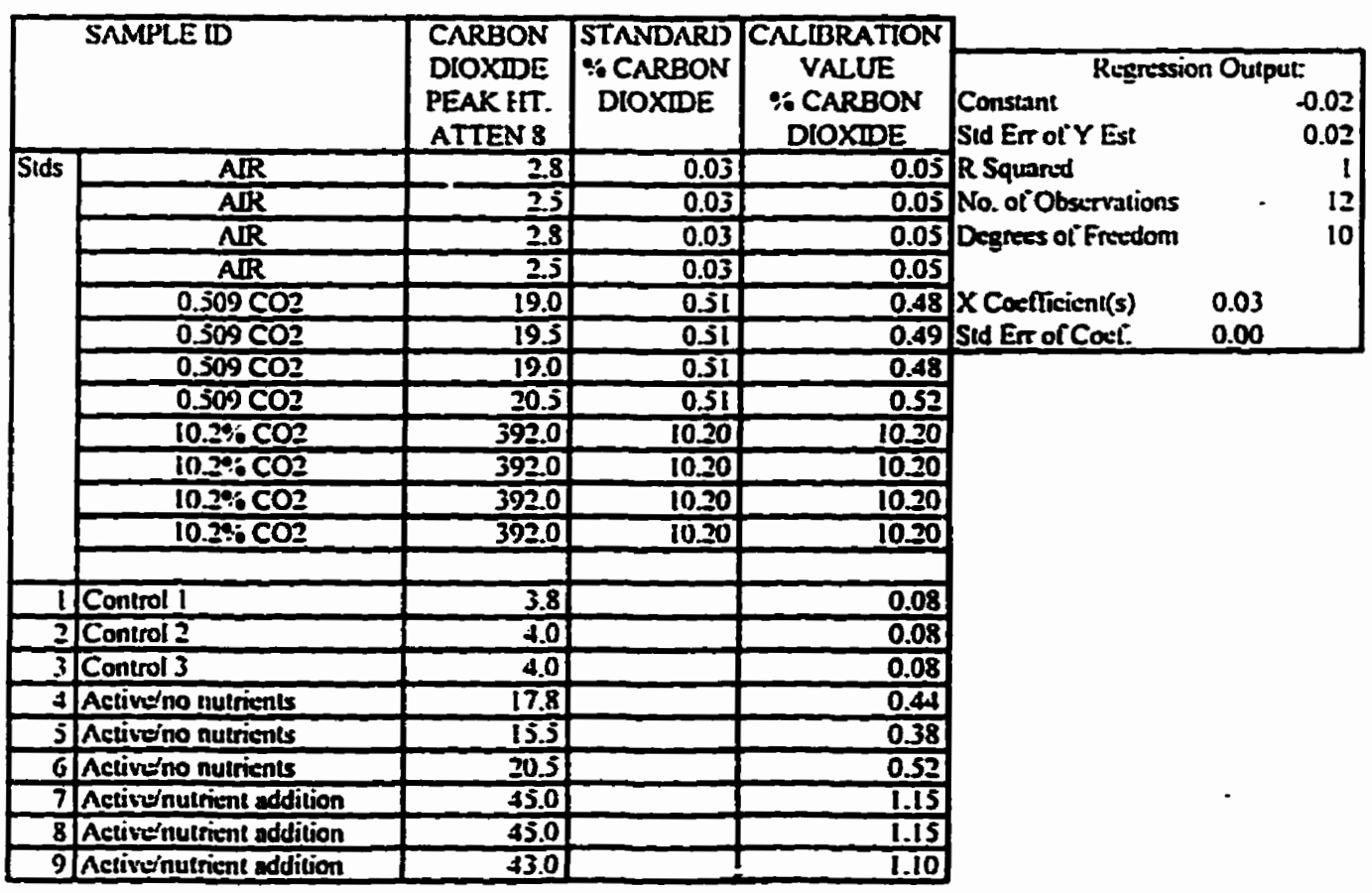


Table 4. Results of oxygen and carbon dioxide measurements in microcosm headspace; results indicate that microcosms remained aerobic for the duration of the experiment and that carbon dioxide accumulated in active microcosms.

\begin{tabular}{|c|c|c|c|c|c|c|}
\hline & & $3 y$ & Day 8 & Jay 15 & Day 29 & ay \\
\hline Oxygen & Control 1 & 21.50 & 22.01 & 21.09 & 19.13 & 19.23 \\
\hline & Control 2 & 20.84 & 21.33 & 21.61 & 19.60 & 18.79 \\
\hline & Control 3 & 19.85 & 20.31 & 21.61 & 19.60 & 19.37 \\
\hline & Active/no nutrients & 20.84 & 27.33 & 21.4 & 19.44 & 18.08 \\
\hline & Active/no mutrients & 21.50 & 22.01 & 20.92 & 18.98 & 18.50 \\
\hline & Active/no mutrients & 21.83 & 22.34 & 20.58 & 18.67 & 19.23 \\
\hline & Active/nutrient adtition & 20.67 & 21.16 & 19.89 & 18.04 & 18.50 \\
\hline & Active/mutrient addition & 21.50 & 22.01 & 20.08 & 18.20 & 20.22 \\
\hline & Active/munient addition & 19.02 & 19.47 & 19.21 & 17.42 & 17.47 \\
\hline Carbon & Control 1 & 0.05 & 0.03 & 0.04 & 0.08 & 0.03 \\
\hline dioxide & Control 2 & 0.03 & 0.03 & 0.05 & 0.08 & 0.03 \\
\hline & Control 3 & 0.04 & 0.03 & 0.03 & 0.08 & 0.03 \\
\hline & Active/ no numrients & 0.18 & 0.21 & 0.32 & 0.44 & 1.02 \\
\hline & Active/no mutrients & 0.18 & 0.24 & 0.29 & 0.38 & 0.79 \\
\hline & Active/no metrients & 0.18 & 0.28 & 0.31 & 0.52 & 0.74 \\
\hline & Active/mutrient addition & 0.12 & 0.26 & 0.41 & 1.15 & 1.20 \\
\hline & Active/nutrient addition & 0.28 & 0.34 & 0.53 & 1.15 & 0.41 \\
\hline & Active/mutrien addition & 0.2 & 0.34 & 0.51 & 1.10 & 1.71 \\
\hline
\end{tabular}




\section{PLATE COUNT METHODS}

(analysis and method write-up by Dr. Barb Butler)

At the conclusion of the microcosm experiment an aliquot of sand from each microcosm was analyzed for total plate counts. As part of this analysis moisture content was also determined. Triplicate samples of wet sand were dispensed into pre-dried aluminum pans, weighed, then dried overnight at $100^{\circ} \mathrm{C}$ and reweighed. Mean values are reported in Table 5.

Table 5. Moisture content

\begin{tabular}{lcc}
\hline Microcosm & Moisture content (\%) & standard deviation \\
\hline Control 1 & 19.76 & 0.12 \\
Control 2 & 19.35 & 0.14 \\
Control 3 & 20.02 & 0.12 \\
Active/no nutrient addition 1 & 18.41 & 0.31 \\
Active/no nutrient addition 2 & 18.87 & 0.24 \\
Active/no nutrient addition 3 & 18.67 & 0.25 \\
Active/nutrient addition 1 & 18.62 & 0.26 \\
Active/nutrient addition 2 & 18.29 & 0.05 \\
Active/nutrient addition 3 & 17.90 & 0.33 \\
\hline
\end{tabular}

To determine plate counts, a suspension of $10 \mathrm{~g}$ (wet $\mathrm{wt}$ ) of sand in $90 \mathrm{~mL}$ of $0.1 \%$ $\mathrm{Na}_{4} \mathrm{P}_{2} \mathrm{O}_{7} 10 \mathrm{H}_{2} \mathrm{O}$ (pH 7.0) was shaken for $10 \mathrm{~min}$ on an orbital shaker operating at $400 \mathrm{rpm}$, then diluted in phosphate-buffered saline $\left(1.18 \mathrm{~g} \mathrm{Na}_{2} \mathrm{HPO}_{4}, 0.22 \mathrm{~g} \mathrm{NaH}_{2} \mathrm{PO}_{4} \cdot \mathrm{H}_{2} \mathrm{O}, 8.5 \mathrm{~g} \mathrm{NaCl}\right.$ per $\mathrm{L}, \mathrm{pH}$ 7.0) and spread on plates of R2A medium. Triplicate plates were prepared for each dilution. Plates were incubated aerobically at room temperature and counts were made at 14 and 24 days and are reported in Table 6.

Table 6. Plate count results, as colony forming units (CFUs)/g dwt.

\begin{tabular}{|c|c|c|c|c|}
\hline \multirow[t]{2}{*}{ Microcosm } & \multicolumn{2}{|c|}{14 days } & \multicolumn{2}{|c|}{24 days } \\
\hline & CFU/g & s.d. & CFU/g & s.d. \\
\hline Control 1 & $0 \times 10^{00}$ & $0 \times 10^{0}$ & $4.2 \times 10^{0^{4}}$ & $5.9 \times 10^{0}$ \\
\hline Control 2 & $8.3 \times 10^{0 \circ}$ & $1.2 \times 10^{t}$ & $8.3 \times 10^{0-}$ & $1.2 \times 10^{1}$ \\
\hline Control 3 & $8.3 \times 10^{0 \circ}$ & $5.9 \times 10^{0}$ & $8.3 \times 10^{0 \circ}$ & $5.9 \times 10^{0}$ \\
\hline Active/no nutrient addition l & $2.4 \times 10^{7}$ & $9.2 \times 10^{6}$ & $2.6 \times 10^{7}$ & $1.2 \times 10^{7}$ \\
\hline Active/no nutrient addition 2 & $1.9 \times 10^{7}$ & $1.5 \times 10^{6}$ & $1.9 \times 10^{7}$ & $1.5 \times 10^{6}$ \\
\hline Active/no nutrient addition 3 & $2.1 \times 10^{7}$ & $2.1 \times 10^{6}$ & $2.1 \times 10^{7}$ & $2.2 \times 10^{6}$ \\
\hline Active/nutrient addition l & $6.1 \times 10^{6}$ & $3.1 \times 10^{6}$ & $9.6 \times 10^{6}$ & $1.3 \times 10^{6}$ \\
\hline Active/nutrient addition 2 & $6.3 \times 10^{6}$ & $9.0 \times 10^{5}$ & $6.5 \times 10^{6}$ & $8.7 \times 10^{5}$ \\
\hline Active/nutrient addition 3 & $6.1 \times 10^{60}$ & $3.5 \times 10^{5}$ & $6.3 \times 10^{6 *}$ & $3.2 \times 10^{5}$ \\
\hline
\end{tabular}

* estimate; some/all plates had fewer than 30 colonies

** some plates contained spreading colonies that obscured/suppressed other cells 


\section{APPENDIX 11 \\ REDOX CHARACTERIZATION - METHODS AND RESULTS}

\section{BACKGROUND}

Groundwater was monitored and sampled for redox-related parameters at times the approximately corresponded to the latter three plume synoptic sampling events (days 626, 1008 and 1357). The most commonly monitored parameter was dissolved oxygen, with two methods used for analysis: CHEMetrics ampule and modification of the Winkler titration. However, data from the former is considered more reliable, and typically yields lower values. Sampling has also been conducted for iron, manganese, nitrate, nitrite, ammonia, sulphate, silica, chloride and methane. Analysis for inorganic parameters was conducted at the Water Quality Laboratory and methane analysis was conducted at the Organic Geochemistry Laboratory, both located at the University of Waterloo. Monitoring for $\mathrm{pH}$ and temperature was conducted at the study site.

\section{METHODS}

Collection of Samples for Laboratory Analysis

1) Methane sample

- Purge $50 \mathrm{~mL}$ with the sampling head fitted with an $18 \mathrm{~mL}$ hypovial

- Clamp the tube connecting the sample head and the piezometer so the groundwater does not nun back down the tube; remove the $18 \mathrm{~mL}$ sample and crimp, leaving no headspace

2) Iron, manganese, ammonium sample

- place a $120 \mathrm{~mL}$ glass hypovial in the sample head and pump the piezometer until the bottle contains at least $80 \mathrm{~mL}$

- remove $20 \mathrm{~mL}$ from the hypovial with a plastic syringe

- filter the $20 \mathrm{~mL}$ into a $20 \mathrm{~mL}$ plastic sample bottle

- acidify with $0.1 \mathrm{~mL} \mathrm{H}_{2} \mathrm{SO}_{4}$ solution

3) Sulphate, nitrate, nitrite, chloride, silica sample

- pour $20 \mathrm{~mL}$ from the glass hypovial directly into $20 \mathrm{~mL}$ plastic sample bottle 


\title{
Dissolved Oxygen by CHEMets Methods
}

\begin{abstract}
A sampling head is used to fill a glass hypovial and to further purge at least $50 \mathrm{~mL}$. The bottle is removed from the head and the CHEMets ampule is placed in the hypovial as rapidly as possible (to minimize potential for atmospheric contamination) and the tip is broken off by pressing the ampule against the bottom of the bottle. After mixing the ampule contents, oxygen concentration is evaluated by colorimetric comparison with standards provided by the company. CHEMetrics ampules are available in the following measurement ranges: 1-12 ppm, 0-1 ppm and 0-100 ppb.
\end{abstract}

\section{Dissolved Oxygen by Modified Winkler Titration}

Purging and sampling is done with $60 \mathrm{~mL}$ plastic syringe which is rinsed with distilled water between samples and re-used. After connecting a syringe to a piezometer tube, $50 \mathrm{~mL}$ of groundwater is extracted and wasted to purge the tube. The syringe is then re-connected to the piezometer and a $50 \mathrm{~mL}$ sample is extracted and titrated in the field by the method provided by Neilsen and Hansen (1992).

\section{RESULTS}

Results of redox-related monitoring are provided in Table 1. 
Table I. Results of groundwater sampling for redox-related parameters. (continued)

Location: 1=croosole plume, 2=background, 3=landfill leachatc plume

\begin{tabular}{|c|c|c|c|c|c|c|c|c|c|c|c|c|c|c|c|c|c|c|}
\hline Piezo. & Dale & $\bar{x}$ & $\overline{\mathbf{Y}}$ & $\bar{z}$ & $\begin{array}{c}\text { Do by } \\
\text { Winkler } \\
\text { (c) }\end{array}$ & $\begin{array}{l}\text { DO by } \\
\text { Chemet } \\
\text { (m, L) }\end{array}$ & $\begin{array}{c}\text { SO4 } \\
\text { (mel) }\end{array}$ & $\begin{array}{l}\text { NO3 } \\
(\mathrm{mel})\end{array}$ & $\begin{array}{l}\mathrm{NO2} \\
(\mathrm{mel})\end{array}$ & $\underset{(m \in R)}{C l}$ & $\begin{array}{l}\text { SiO2 } \\
(m \in L)\end{array}$ & \begin{tabular}{|c|} 
Fe \\
$(m \in L)$
\end{tabular} & $\underset{(m / h)}{M n}$ & \begin{tabular}{|c|}
$\mathrm{NH} 4$ \\
$(\mathrm{~m} / \mathrm{h})$
\end{tabular} & $\begin{array}{l}\text { Cl14 } \\
\text { (pppb) }\end{array}$ & $\begin{array}{c}\text { Temp } \\
(\operatorname{deg} C)\end{array}$ & $\mathrm{pH}$ & Location \\
\hline $9-0-6$ & 02.Jun-95 & $\underline{0}$ & 15.5 & 3.3 & & 0.06 & & & & & & & & & & & & \\
\hline $9-0-6$ & 14-Jun-94 & $\overline{0}$ & 15.5 & 3.3 & 0.22 & & & & & & & & & & & 13.5 & & \\
\hline $9-0-7$ & $26-0 x-93$ & 0 & 15.5 & 3.6 & 0.27 & & 13.5 & $<0.01$ & 0.45 & & & 0.97 & 0.09 & 0.51 & 2 & & & \\
\hline $9-0-7$ & $02 \cdot \mathrm{Jun}-95$ & 0 & 15.5 & 3.6 & 0.132 & 0.04 & 21.8 & 40.05 & $<0.05$ & 1.51 & 15.3 & 0.48 & 0.06 & 0.39 & 10 & 12.9 & 7.42 & \\
\hline $9-0.7$ & 14-Jun-94 & 0 & 15.5 & 3.6 & 0.19 & & II.1. & 40.01 & $\$ 0.01$ & & & 0.53 & 0.06 & 40.1 & 11.4 & 14.2 & & \\
\hline $9-0-6$ & $26-0 \mathrm{et}-93$ & 0 & 15.5 & 3.3 & 0.56 & & 14.9 & $<0.01$ & 0.38 & & & 0.45 & 0.12 & $<0.05$ & 2.1 & & & \\
\hline $9-0-4$ & $02-$ Jun-95 & 0 & 15.5 & 2.7 & & 0.06 & & & & & & & & & & & & \\
\hline $9-0-4$ & 14-Jun-94 & 0 & 15.5 & 2.7 & 0.24 & & & & & & & & & & & 13.3 & & \\
\hline $9-0.5$ & $26-0 \mathrm{ct}-93$ & 0 & 15.5 & 3 & 0.35 & & 15.2 & $<0.01$ & 2.7 & & & 0.66 & 0.21 & $<0.05$ & 1.9 & & & \\
\hline $9-0.5$ & 02-Jun-95 & 0 & 15.5 & 3 & & 0.06 & & & & & & & & & & & & \\
\hline 90.5 & 14-Jun-94 & 0 & 15.5 & 3 & 0.15 & & & & & & & & & & & 14.2 & & I \\
\hline $9-0-10$ & 14.Jun-94 & 0 & 15.5 & 4.5 & 0.41 & & & & & & & & & & & 13.6 & & \\
\hline $9-0.10$ & $26-0 \mathrm{ct}-93$ & 0 & 15.5 & 4.5 & 0.61 & & 12.6 & $<0.01$ & 0.28 & & & 0.06 & 0.04 & $<0.05$ & 1.8 & & & \\
\hline $9-0-10$ & 02-Jun-95 & 0 & 15.5 & 4.5 & & 0.06 & & & & & & & & & & & & \\
\hline 27.1 .7 & 14-Jun-94 & 2 & 49 & 3 & 0.2 & & & & & & & & & & & & & \\
\hline $27.1-7$ & 02-5un-95 & 2 & 49 & 3 & & 0.2 & & & & & & & & & & & & \\
\hline $9-0.9$ & 02.Jun-95 & 0 & 15.5 & 4.2 & & 0.08 & & & & & & & & & & & & \\
\hline $9-0.8$ & 14-Jun-94 & 0 & 15.5 & 3.9 & 0.18 & & & & & & & & & & & 14.2 & & \\
\hline $9-0-8$ & $26-0 \mathrm{ct}-93$ & 0 & 15.5 & 3.9 & 0.38 & & 12.9 & $\leq 0.01$ & 80.01 & & & 0.62 & 0.07 & $<0.05$ & 1.9 & & & \\
\hline $9-0-8$ & $02-\sqrt{J u n-95}$ & $\underline{0}$ & 15.5 & 3.9 & & 0.06 & & & & & & & & & & & & \\
\hline $9-0-9$ & 14-Jun-94 & 0 & 15.5 & 4.2 & 0.3 & & & & & & & & & & & 14.2 & & \\
\hline $9-0-9$ & $26-0$ ㅇt-93 & 0 & 15.5 & 4.2 & 0.56 & & 12.3 & $<0.01$ & 0,94 & & & 0.3 & 0.08 & 0.19 & 2 & & & \\
\hline $34-4$ & 14-Jun-94 & 2.52 & 13.5 & 2.7 & 0 & 0.01 & & & & & & & & & & 11.4 & & \\
\hline 34-5 & 02.Jun-95 & 2.52 & 13.5 & 2.4 & 0.074 & 0.03 & 13.1 & 16.4 & $<0.05$ & 2.54 & 10.6 & 0.67 & 0.07 & 0.31 & & 10.1 & 7.33 & \\
\hline 344 & 02-Jun-95 & 2.52 & 13.5 & 2.7 & 0.058 & 0 & & & & & & & & & $<0.1$ & & & \\
\hline $34-1$ & 14-Jun-94 & 2.52 & 13.5 & 3.6 & 0.15 & 0.02 & 11.4 & $<0.01$ & 40.01 & & & 0.08 & 0.04 & $<0.1$ & 1.8 & 12 & & \\
\hline $34-1$ & $26-0 \mathrm{ct} \cdot 93$ & 2.52 & 13.5 & 3.6 & 0.38 & & 12.1 & $<0.01$ & $<0.01$ & & & 0.1 & 0.05 & 0.1 & 2 & & & \\
\hline $34-5$ & 14-Jun-94 & 2.52 & 13.5 & 2.4 & 0.12 & 0.02 & 9.8 & $\Delta 0.01$ & $<0.01$ & & & 0.45 & 0.08 & 40.1 & 67.1 & 11.6 & & \\
\hline 6.1 .7 & 26-Jum-93 & 1.5 & 8 & 3.2 & 0.43 & & & & & & & & & & & & & \\
\hline $6-1-1$ & 26-Jun-93 & 1.5 & 8 & 2 & 0.17 & & & & & & & & & & & & & \\
\hline $31-1-1$ & 14-Jun-94 & 2 & 57 & 1.8 & 0.24 & & & & & & & & & & & & & \\
\hline
\end{tabular}




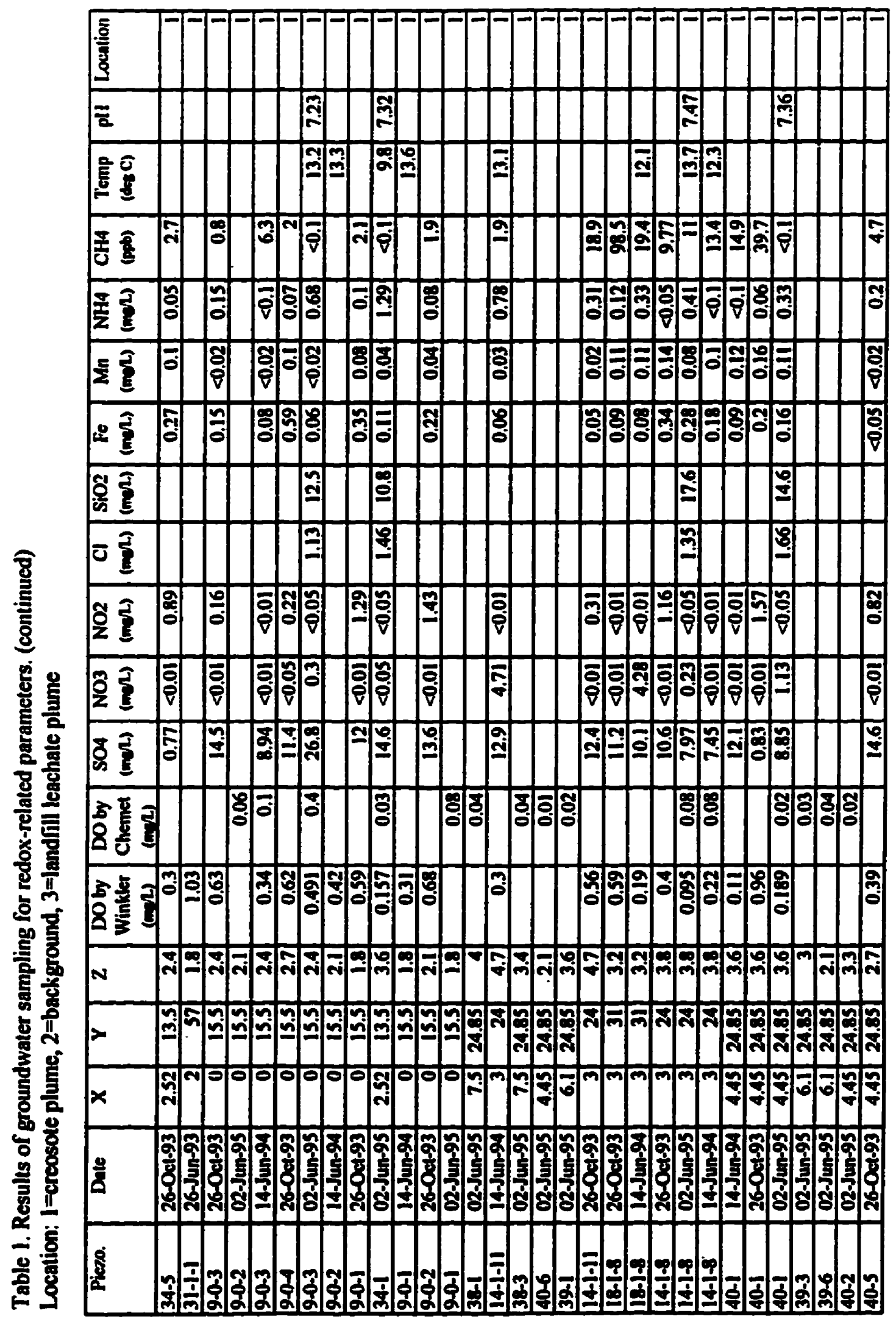




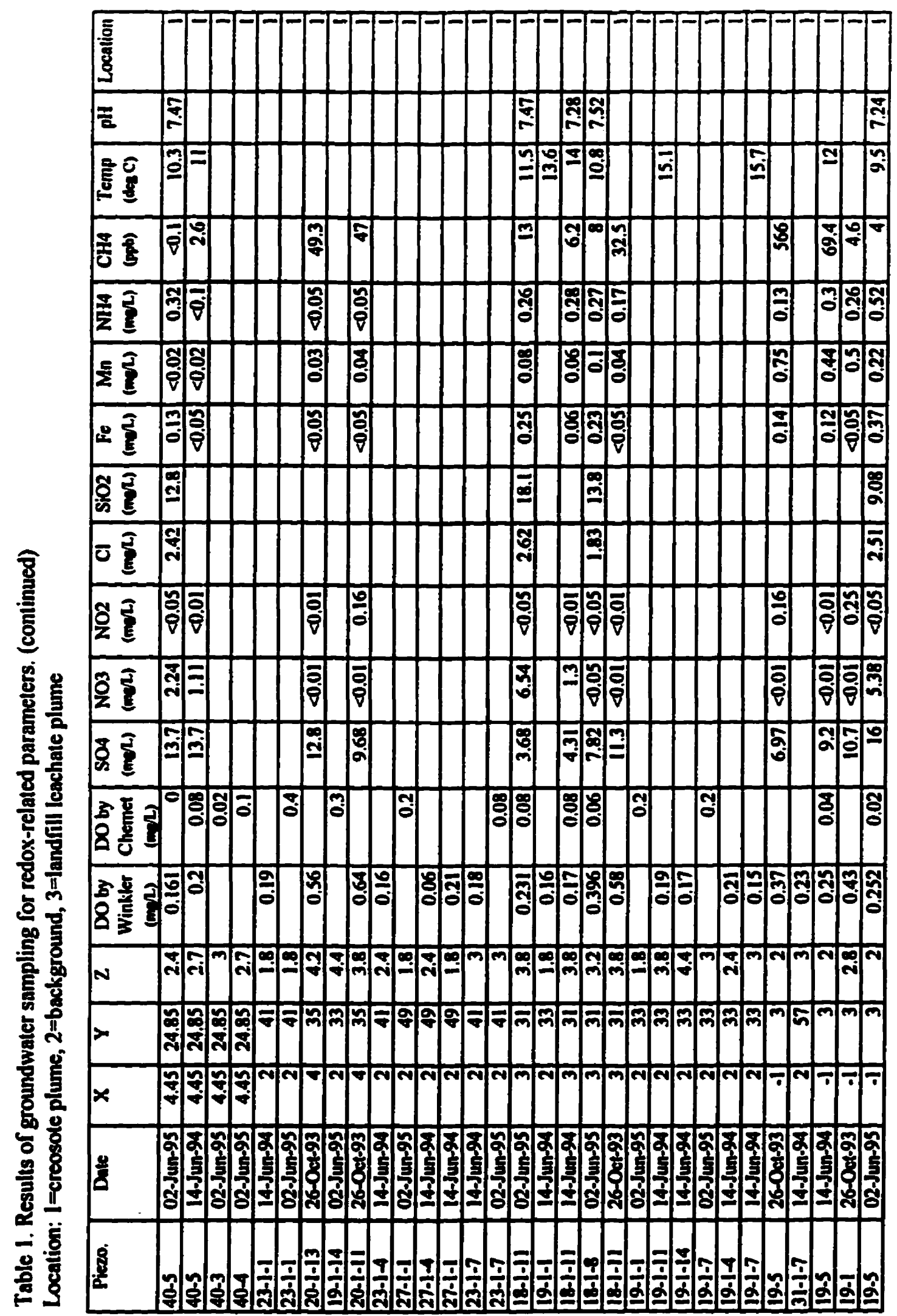


Table 1. Results of groundwater sampling for redox-related paramelers. (continued)

Location: 1=creosote plume, 2=background, 3=landifll leachate plume

\begin{tabular}{|c|c|c|c|c|c|c|c|c|c|c|c|c|c|c|c|c|c|c|}
\hline Piezo. & Dale & $\overline{\mathbf{x}}$ & $\mathbf{Y}$ & 2 & $\begin{array}{c}\text { DO by } \\
\text { Winkler } \\
(\mathrm{n} / \mathrm{L})\end{array}$ & $\begin{array}{l}\text { DO by } \\
\text { Chemet } \\
(m / 2)\end{array}$ & $\begin{array}{c}504 \\
(\mathrm{mat})\end{array}$ & $\begin{array}{l}\mathrm{NO3} \\
(\mathrm{m} / \mathrm{L})\end{array}$ & $\begin{array}{l}\text { NO2 } \\
(m / 2)\end{array}$ & $\underset{(m \in L)}{C \mathrm{Cl}}$ & $\begin{array}{l}\mathrm{SiO2} \\
(\mathrm{mel})\end{array}$ & $\begin{array}{c}F_{0} \\
(\text { mets })\end{array}$ & $\begin{array}{c}M n \\
(m / L)\end{array}$ & \begin{tabular}{|l|} 
NH4 \\
$(m / L)$
\end{tabular} & $\begin{array}{l}\text { CH4 } \\
\text { (ppos) }\end{array}$ & $\begin{array}{l}\text { Temp } \\
(\log \mathrm{C})\end{array}$ & pll & Location \\
\hline $12-7$ & 02-Jun-95 & 1.3 & 0.95 & 2.93 & 0.219 & 0.01 & 11.6 & 2.64 & 0.07 & 1.47 & 9.7 & $<0.05$ & 0.37 & 0.56 & 37 & 9 & 7.25 & \\
\hline $\mathbf{1 2 . 1 3}$ & 02-Jun-95 & 1.3 & 0.95 & 1.73 & 0.107 & 0.06 & & & & & & & & & & & & \\
\hline 12.13 & 14-Jun-94 & 1.3 & 0.95 & 1.73 & $\Delta 0.069$ & 0 & & & & & & & & & & 10 & & \\
\hline $12-10$ & 14-Jun-94 & 1.3 & 0.95 & 2.33 & 0.11 & & & & & & & & & & & 12.6 & & \\
\hline $12-7$ & 14-Jun-94 & 1.3 & 0.95 & 2.93 & 0.14 & & 11.2 & 40.01 & $<0.01$ & & & 0.07 & 0.49 & $<0.1$ & 15 & 10.9 & & \\
\hline $12 \cdot 10$ & 02-Jun-95 & 1.3 & 0.95 & 2.33 & 0.346 & 0.04 & & & & & & & & & & & & \\
\hline 31.1 .7 & $26-\sqrt{\sin -93}$ & 2 & 57 & 3 & 1.69 & & & & & & & & & & & & & \\
\hline 27.2 & $26-04-93$ & 1 & 6.8 & 3.3 & 0.35 & & 11.3 & $<0.01$ & $\pi$ & & & $<0,05$ & 0.06 & 0.1 & 5.1 & & & \\
\hline $27-2$ & 14-Jun-94 & 1 & 6.8 & 3.3 & 0.16 & & 11.3 & $<0.01$ & $<0,01$ & & & 0.05 & 0.05 & $<0.1$ & 156 & 11.4 & & \\
\hline 27.5 & 02-Jun-95 & 1 & 6.8 & 2.4 & 0.078 & $\overline{0}$ & 15.4 & $<0.05$ & $<0.05$ & 2.44 & 10 & 0.07 & 0.15 & 0.37 & 3 & 9.1 & 6.84 & \\
\hline 27.5 & 26-0c1-93 & 1 & 6.8 & 2.4 & 0.39 & & 10.7 & $<0.01$ & 0.37 & & & 40.05 & 0,09 & 0.08 & 13.4 & & & \\
\hline 27.5 & 14-Jum-9n| & 1 & 6.8 & 2.4 & 0.15 & 0.03 & 9.61 & 1.5 & 80.01 & & & 0.09 & 0.09 & 0.32 & 503 & 11.2 & & \\
\hline $27-2$ & 02.Jum-95 & ] & 6.8 & 3.3 & 0.144 & 0.01 & 14.4 & 2.79 & 40.05 & 1.77 & 10.8 & 0.1 & 0.05 & 0.29 & 2 & 9.1 & 7.32 & \\
\hline $19-1$ & $02-\sqrt{u n}-95$ & -1 & 3 & 2.8 & 0.144 & 0.04 & 11 & 29.3 & $<0.05$ & 2.26 & 9.17 & 0.1 & 0.42 & 0.5 & 80.1 & 8.9 & 7.39 & \\
\hline $19-1$ & 14-Jun-94 & -1 & 3 & 2.8 & 0.24 & 0.1 & 11.4 & $\Delta .01$ & 40.01 & & & $<0,05$ & 0.57 & 4.1 & 0.4 & 11.2 & & \\
\hline $3-04$ & 26-Jun-93 & 0 & 3.5 & 2.4 & 0.31 & & & & & & & & & & & & & \\
\hline $3-0-11$ & 26-Jun-93 & $\overline{0}$ & 3.5 & 3.8 & 0.45 & & & & & & & & & & & & & \\
\hline $3-0.7$ & $26-\sqrt{\ln 2-93}$ & $\underline{0}$ & 3.5 & 3 & 0.29 & & & & & & & & & & & & & \\
\hline 604 & 14-Jum-94 & $\underline{0}$ & 8 & 2.6 & 0.33 & & & & & & & & & & & 10.7 & 7.25 & \\
\hline 6-04 & 02.Jun-9s & $\overline{0}$ & 8 & 2.6 & 0.305 & 0.4 & & & & & & & & & & & & \\
\hline $6-04$ & $26-\operatorname{Jun}-93$ & 0 & 8 & 2.6 & 1.03 & & & & & & & & & & & & & \\
\hline $6-0-1$ & 02-Jun-95 & 0 & 8 & 2 & 1.2 & 0.6 & & & & & & & & & & & & \\
\hline $60-2$ & 14-Jun-94 & $\overline{0}$ & 8 & 2.2 & 0.21 & & & & & & & & & & & 11.3 & & \\
\hline $6-0.7$ & 26-Jun-93 & 0 & 8 & 3.2 & 0.48 & & & & & & & & & & & & & \\
\hline $6-0-10$ & 14-Jen-91 & 0 & 8 & 3.8 & 0.37 & & & & & & & & & & & 10 & & \\
\hline $6-0-11$ & 02-Jun-95 & $\underline{0}$ & 8 & 4 & 0.453 & 0.6 & & & & & & & & & & & & \\
\hline $3-7$ & 02-Jun-95 & 1.3 & 0.5 & 2.19 & & 0.03 & & & & & & & & & & & & \\
\hline $6-0-7$ & 14-Jun-29 & $\underline{\mathbf{0}}$ & 8 & 3.2 & 0.43 & & & & & & & & & & & II & & \\
\hline $6-0.7$ & 02-Jun-95 & $\underline{0}$ & 8 & 3.2 & 0.503 & 0.2 & & & & & & & & & & & & \\
\hline $6-0-1$ & 26-Jun-93 & $\underline{0}$ & 8 & 2 & 1.1 & & & & & & & & & & & & & \\
\hline $3 !-1-1$ & 02-Jun-95 & 2 & 57 & 1.8 & & 0.3 & & & & & & & & & & & & \\
\hline
\end{tabular}


Table 1. Results of groundwater sampling for redox-related parameters. (continued)

Location: I=creosole plume, 2=background, 3=landfill leachate plume

\begin{tabular}{|c|c|c|c|c|c|c|c|c|c|c|c|c|c|c|c|c|c|c|}
\hline Piezo. & Dale & $\bar{x}$ & $\bar{Y}$ & $\overline{2}$ & $\begin{array}{l}\text { DO by } \\
\text { Winklet } \\
\left(\mathrm{m}^{2} \Omega\right)\end{array}$ & $\begin{array}{l}\text { Do by } \\
\text { Chemet } \\
(\mathrm{m} / \mathrm{L})\end{array}$ & $\begin{array}{c}\mathrm{SO4} \\
(\mathrm{mel})\end{array}$ & $\begin{array}{l}\text { NO3 } \\
(m / L)\end{array}$ & $\begin{array}{l}\text { NO2 } \\
(m, L / 2)\end{array}$ & $\underset{(m / L)}{\mathbf{C l}}$ & \begin{tabular}{|l|} 
SiO2 \\
$(m / 2)$
\end{tabular} & $\underset{(m e / L)}{F}$ & $\begin{array}{c}M n \\
(m \in L)\end{array}$ & \begin{tabular}{|l|} 
NH4 \\
$(m \in L)$
\end{tabular} & $\begin{array}{l}\text { Clt4 } \\
\text { (ppob) }\end{array}$ & $\begin{array}{l}\text { Temp } \\
\text { (dos C) }\end{array}$ & $\mathrm{pHH}$ & Localion \\
\hline 6-NI-1 & 02-Jun-95 & -1.5 & 8 & 2.1 & 0.462 & 0.3 & & & & & & & & & & & & 1 \\
\hline $31-1-4$ & 14-Jun-94 & 2 & 57 & 2.4 & 0.36 & & & & & & & & & & & & & \\
\hline$\overline{3-2}$ & 02-Jum-95 & 1.3 & 0.5 & 3.19 & & 0.6 & & & & & & & & & & & & $\frac{1}{1}$ \\
\hline 3 & 02-Jun-95 & 1.3 & 0.5 & 2.99 & & 0.6 & & & & & & & & & & & & \\
\hline 6-N1-7 & \begin{tabular}{|c|}
$02-J u n-95$ \\
\end{tabular} & -1.5 & 8 & 3.3 & 0.429 & 0.3 & & & & & & & & & & & & \\
\hline 3.5 & $02 \cdot J u n-95$ & 1.3 & 0.5 & 2.59 & & 0.3 & & & & & & & & & & & & \\
\hline
\end{tabular}

Disoolved Creopone Plume

\begin{tabular}{|l|r|r|r|r|r|r|r|r|r|r|r|r|r|}
\hline $\operatorname{mean}$ & 0.34729 & 0.12905 & 11.568 & 1.5066 & 0.2757 & 1.9264 & 12.49 & 0.198 & 0.13 & 0.211 & 35.65 & 11.9591 & 7.323 \\
\hline $\mathrm{n}$ & 101 & 63 & 53 & 53 & 53 & 14 & 14 & 53 & 53 & 53 & 53 & 44 & 16 \\
\hline $\mathrm{s.d}$ & 0.26816 & 0.16312 & 4.1864 & 4.694 & 0.5367 & 0.5179 & 3.008 & 0.217 & 0.161 & 0.249 & 103.82 & 1.78473 & 0.159 \\
\hline $\min$ & 0 & 0 & 0.77 & 0 & 0 & 1.13 & 9.08 & 0 & 0 & 0 & 0 & 8.9 & 6.84 \\
\hline $\max$ & 1.69 & 0.6 & 26.8 & 29.3 & 2.7 & 2.62 & 18.1 & 0.97 & 0.75 & 1.29 & 566 & 15.7 & 7.52 \\
\hline
\end{tabular}

\begin{tabular}{|c|c|c|c|c|c|c|c|c|c|c|c|c|c|c|c|c|c|c|}
\hline N1-0.7 & \begin{tabular}{|l|}
$26-J u n-93$ \\
\end{tabular} & 0 & -0.5 & 3 & 2.62 & & & & & & & - & & L & & & & 2 \\
\hline N1-0-14 & \begin{tabular}{|l|}
$20-J u n-93$ \\
\end{tabular} & $\underline{\mathbf{0}}$ & -0.5 & 4.4 & 2.1 & & & & & & & & & & & & & 7 \\
\hline $13-\sqrt{4} \cdot 1$ & \begin{tabular}{|l|}
$26-J u n-93$ \\
\end{tabular} & .10 .5 & 22.5 & 1.8 & 0.62 & & & & & & & & & & & & & \\
\hline N1-0-11 & 26-Jun-93 & $\underline{0}$ & -0.5 & 3.8 & 2.91 & & & & & & & & & & & & & \\
\hline 13-N4-14 & 26.Jun-93 & -10.5 & 22.5 & 4.4 & 3.67 & & & & & & & & & & & & & \\
\hline 13-N2-1 & 26-Jun-93 & 4.5 & 22.5 & 1.8 & 0.14 & & & & & & & & & & & & & \\
\hline 13-N2-7 & \begin{tabular}{|l|}
$26-J u n-93$ \\
\end{tabular} & -4.5 & 22.5 & 3 & 1.96 & & & & & & & & & & & & & \\
\hline $13-\sqrt[N 4-7]{ }$ & \begin{tabular}{|l|}
$26-J \operatorname{sen}-93$ \\
\end{tabular} & .10 .5 & 22.5 & 3 & 2.43 & & & & & & & & & & & & & 2 \\
\hline $12-3$ & \begin{tabular}{|l|}
$02-J u n-95$ \\
\end{tabular} & $\overline{1.3}$ & 0.95 & 3.73 & 2.18 & I & & & & & & & & & & & & \\
\hline $12-3$ & 14-Jun-94 & 1.3 & 0.95 & 3.73 & 2.21 & & & & & & & & & & & 10.3 & & \\
\hline 12.1 & 02-Jun-95 & 1.3 & 0.95 & 4.13 & 1.47 & & & & & & & & & & & & & \\
\hline 12.1 & 14-Jun-94 & 1.3 & 0.95 & 4.13 & 1.38 & & & & & & & & & & & 11.3 & 6.45 & \\
\hline $13-\sqrt{2}-14$ & \begin{tabular}{|l|} 
26.Jun-933 \\
\end{tabular} & 4.5 & 22.5 & 4.4 & 1.41 & & & & & & & & & & & & & \\
\hline N1-04 & 26-Jun-93 & $\overline{0}$ & $-0,5$ & 2.4 & 3.31 & & & & & & & & & & & & & \\
\hline NI-0-1 & \begin{tabular}{|l|}
$26-J u n-93$ \\
\end{tabular} & $\underline{\mathbf{0}}$ & -0.5 & 1.8 & 3.77 & & & & & & & & & & & & & \\
\hline $3-0-14$ & 26-Jun-93 & 0 & 3.5 & 4.4 & 1.43 & & & & & & & & & & & & & \\
\hline $6-\sqrt{2}-1$ & 26-Jun-93 & -3 & 8 & 2 & 4.1 & & & & & & & & & & & & & \\
\hline
\end{tabular}




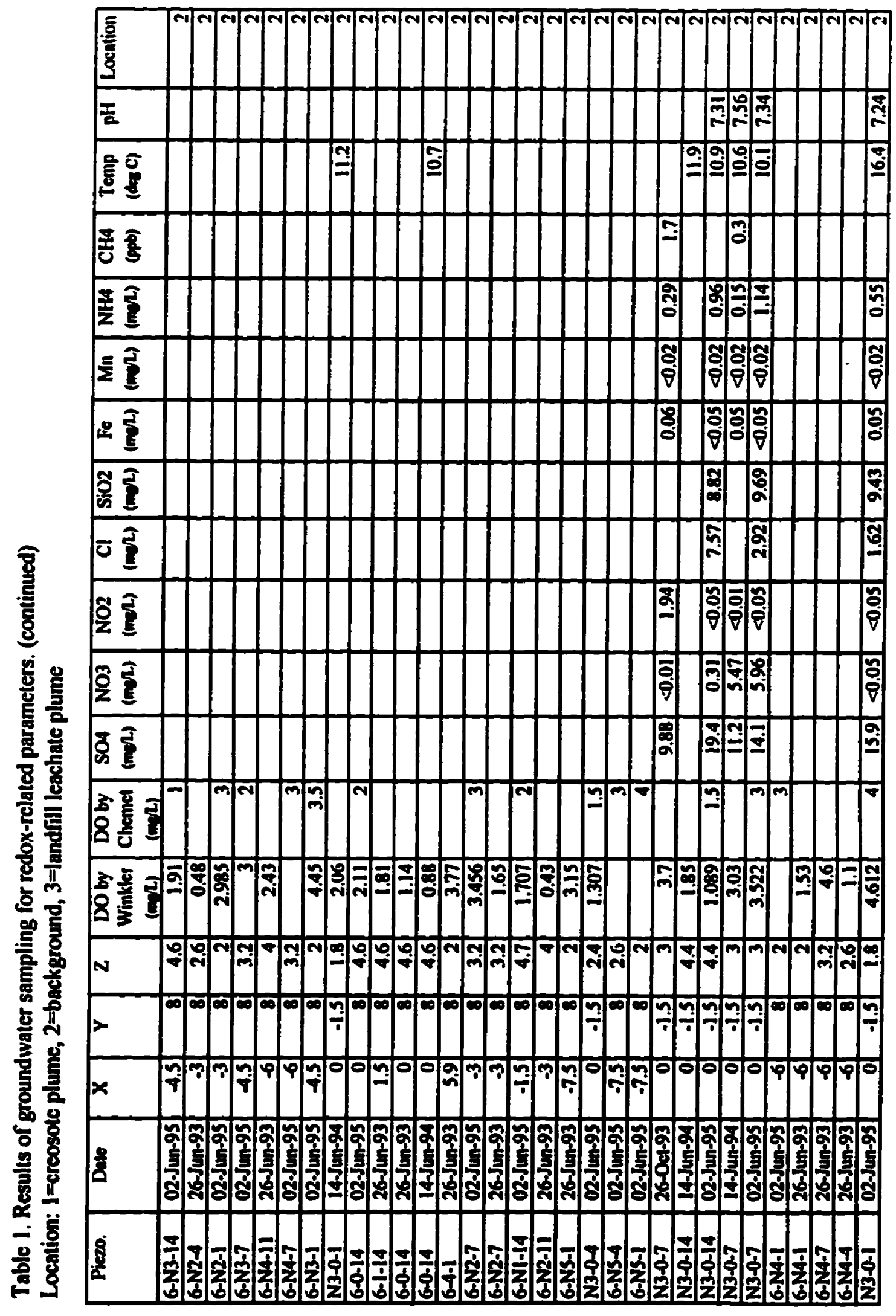


Table 1. Results of groundwater sampling for redox-related parameters. (continued)

Location: l=creosole plume, 2=background, 3=landfill leachate plume

\begin{tabular}{|c|c|c|c|c|c|c|c|c|c|c|c|c|c|c|c|c|c|c|}
\hline Piczo. & Dale & $\bar{x}$ & $\bar{Y}$ & $\bar{Z}$ & $\begin{array}{l}\text { DO by } \\
\text { Winkler } \\
(n+1)\end{array}$ & $\begin{array}{l}\text { DO by } \\
\text { Chernet } \\
(\mathrm{m} / \mathrm{l})\end{array}$ & $\begin{array}{c}\mathrm{SO} 4 \\
(\operatorname{men})\end{array}$ & $\begin{array}{l}\mathrm{NO}^{\mathrm{NO}} \\
\text { (mel) }\end{array}$ & $\begin{array}{l}\mathrm{NO2} \\
(\mathrm{m} / \mathrm{L})\end{array}$ & $\underset{(m \in R)}{C}$ & $\begin{array}{l}\text { SiO2 } \\
(\text { mel })\end{array}$ & $\begin{array}{c}\mathrm{Fe} \\
(\mathrm{m} / \mathrm{l})\end{array}$ & $\begin{array}{c}M n \\
(m \in R)\end{array}$ & $\begin{array}{l}\mathrm{NH4} \\
(\mathrm{m} / \mathrm{l}) .\end{array}$ & $\begin{array}{l}\mathrm{Cl} / 4 \\
\text { (ppb) }\end{array}$ & $\begin{array}{l}\text { Teinp } \\
(\operatorname{deg} C)\end{array}$ & plt & Location \\
\hline 6-N5-7 & $26-\sqrt{u n-93}$ & -7.5 & & 3.2 & 2.38 & & & & & & & & & & & & & $\overline{2}$ \\
\hline 6-N54 & 26-Jun-93 & -7.5 & & 2.6 & 1.74 & & & & & & & & & & & & & 2 \\
\hline 6-NS-11 & 26-Jun-93 & -7.5 & & $\overline{4}$ & 2.48 & & & & & & & & & & & & & $\overline{2}$ \\
\hline 6.N5-9 & 02 -Jun-95 & -7.5 & & 3.6 & & 3 & & & & & & & & & & & & $\frac{7}{2}$ \\
\hline
\end{tabular}

\begin{tabular}{|l|r|r|r|r|r|r|r|r|r|r|r|r|r|}
\hline Background \\
\hline $\operatorname{mean}$ & 2.29932 & 2.47222 & 14.096 & 2.348 & 0.388 & 4.0367 & 9.313 & 0.032 & 0 & 0.618 & 1 & 11.4889 & 7.18 \\
\hline count & 47 & 18 & 5 & 5 & 5 & 3 & 3 & 5 & 5 & 5 & 2 & 9 & 5 \\
\hline S.d. & 1.14637 & 0.99221 & 3.793 & 3.0811 & 0.8676 & 3.1282 & 0.447 & 0.029 & 0 & 0.424 & 0.9899 & 1.92058 & 0.425 \\
\hline $\min$ & 0.14 & 1 & 9.88 & 0 & 0 & 1.62 & 8.82 & 0 & 0 & 0.15 & 0.3 & 10.1 & 6.45 \\
\hline $\max$ & 4.612 & 4 & 19.4 & 5.96 & 1.94 & 7.57 & 9.69 & 0.06 & 0 & 1.14 & 1.7 & 16.4 & 7.56 \\
\hline
\end{tabular}

\begin{tabular}{|c|c|c|c|c|c|c|c|c|c|c|c|c|c|c|c|c|c|c|}
\hline $23-1-13$ & 14.Jun-94 & 2 & 41 & 4.2 & 0.25 & & 27.9 & 1.39 & $<0.01$ & & & 0.18 & $<0.02$ & $\Delta 0.1$ & 14.6 & & & 3 \\
\hline $23-1-13$ & $26-0$ ct-93 & 2 & 41 & 4.2 & 0.58 & & 45.1 & $<0.01$ & 0.8 & & & 0.19 & 0.02 & 40.05 & 16.5 & & & 3 \\
\hline $23-1 \cdot 13$ & 02-Jun-95 & $\overline{2}$ & 41 & 4.2 & 0.429 & 0.08 & 44.5 & $<0,05$ & $<0,05$ & 23.4 & 8.33 & 0.53 & 0.04 & 0.4 & 50 & 15 & 7.32 & 3 \\
\hline $31-1-11$ & 02-Jun-95 & $\overline{2}$ & 57 & $\overline{3}$ & 0.51 & 0.1 & 55.4 & 38.4 & $<0.05$ & 33.1 & 7.94 & 0.34 & $<0,02$ & 0.45 & 17 & & & 3 \\
\hline $31-1-11$ & 14-Jun-94 & 2 & 57 & 3.8 & 0.24 & & & & & & & & & & & & & 3 \\
\hline $23-1-11$ & 02-Jun-95 & $\overline{2}$ & 41 & 3.8 & 0.379 & 0.15 & 22.5 & 32.1 & $<0.05$ & 11.2 & 9.35 & 0.19 & 40.02 & 0.3 & 17 & 12.7 & 7.46 & 3 \\
\hline 31.1 .14 & 02.Jun-95 & $\overline{2}$ & 57 & 4.4 & 0.216 & 0.08 & 122 & 0.33 & 40.05 & 36 & 8.3 & 1.64 & 0.06 & 0.52 & 9 & & & 3 \\
\hline 31.1 .14 & $26 \cdot \sqrt{\ln -93}$ & $\overline{2}$ & 57 & 4.4 & 0.33 & & & & & & & & & & & & & 3. \\
\hline $31-1-14$ & 14-Jun-94 & 2 & 57 & 4.4 & 0.11 & & & & & & & & & & & & 7.15 & 3 \\
\hline 23.1 .11 & 14-Jun-94 & $\overline{2}$ & 41 & 3.8 & 0.14 & 0.04 & 20.1 & $<0.01$ & 80.01 & & & 0.08 & $<0.02$ & 80.1 & 7 & & & 3 \\
\hline $23-1-11$ & $26-0$ c4-93 & 2 & 41 & 3.8 & 0.62 & & 19.3 & $<0.01$ & 0.66 & & & 0.05 & $<0.02$ & 0.05 & $\overline{6.6}$ & & & $\frac{5}{3}$ \\
\hline $9 \cdot 0.12$ & $02-\sqrt{\mathrm{m} n-95}$ & $\underline{0}$ & 15.5 & 5.1 & & 0.6 & & & & & & & & & & & & 3 \\
\hline $9-0-13$ & $26-0 \mathrm{cl}-93$ & 0 & 15.5 & 5.4 & 0.31 & & 82.2 & $<0.01$ & 0.55 & & & 2.04 & 0.11 & 0.07 & 21.2 & & & 3 \\
\hline $9-0-11$ & $26-0 \mathrm{ct-93}$ & $\underline{0}$ & 15.5 & 4.8 & 0.53 & & 47.2 & $<0.01$ & 0.61 & & & $<0.05$ & $<0,02$ & $<0.05$ & 17 & & & 3 \\
\hline $9-0-11$ & 02-Jun-95 & $\overline{0}$ & 15.5 & 4.8 & 0.264 & 0.06 & 38.2 & 9.14 & 80.05 & 18 & 8.58 & 0.1 & $<0.02$ & 0.41 & & $\overline{13.5}$ & 7.17 & 3 \\
\hline $9-0.11$ & 14.Jun-94 & $\overline{0}$ & 15.5 & 4.8 & 0.34 & & 23,6 & 1.91 & 80.01 & & & $<0.05$ & $<0,02$ & 0.29 & 70.9 & 14.2 & & 3 \\
\hline $9-0.14$ & 14-Jun-94 & 0 & 15.5 & 5.7 & $<0.069$ & 0.08 & & & & & & & & & & 15.2 & & 3 \\
\hline $9-0-14$ & 02-Jun-95 & $\overline{0}$ & 15.5 & 5.7 & & 0.06 & & & & & & & & & & & & 3 \\
\hline $9-0-13$ & 14-Jun-94 & 0 & 15.5 & 5.4 & 0.1 & & & & & & & & & & & & & 3 \\
\hline
\end{tabular}


Table 1. Results of groundwater sampling for redox-rclated parameters. (continued)

Location: $1=$ creosole plume, 2 =background, $3=$ landfill leachatc plume

\begin{tabular}{|c|c|c|c|c|c|c|c|c|c|c|c|c|c|c|c|c|c|c|}
\hline Piezo. & Dale & $\bar{x}$ & $\bar{Y}$ & $\overline{\mathbf{z}}$ & $\begin{array}{l}\text { DO by } \\
\text { Winkker } \\
(1,2)\end{array}$ & $\begin{array}{l}\text { DO by } \\
\text { Chemet } \\
(\mathrm{m} / \mathrm{L})\end{array}$ & $\begin{array}{c}\mathrm{SO} 4 \\
(\mathrm{~m} / \mathrm{L})\end{array}$ & $\begin{array}{l}\text { NO3 } \\
(m, L)\end{array}$ & $\begin{array}{l}\mathrm{NO2} \\
(\mathrm{m}=\mathrm{L})\end{array}$ & $\underset{(m \in L)}{\mathbf{C l}}$ & $\begin{array}{l}\mathrm{SiO2} \\
(\mathrm{mel})\end{array}$ & $\begin{array}{c}\text { Fe } \\
(m \in / L)\end{array}$ & $\begin{array}{c}\mathrm{Mn} \\
(m / L)\end{array}$ & $\begin{array}{l}\text { NH4 } \\
(m \in L)\end{array}$ & $\begin{array}{l}\mathrm{CH4} \\
\text { (ppp) }\end{array}$ & $\begin{array}{l}\text { Temp } \\
(\operatorname{deg} C)\end{array}$ & pll & Location \\
\hline $9-0.13$ & 02.Jun-95 & $\overline{\mathbf{0}}$ & 15.5 & 5.4 & & 0.06 & & & & & & & & & & & & $\sqrt[3]{3}$ \\
\hline $9-0.12$ & $26-0 \mathrm{ct}-93$ & $\overline{0}$ & 15.5 & 5.1 & 0.74 & & $\overline{18.3}$ & 40.01 & 0.25 & & & $<0,05$ & $<0,02$ & $<0.05$ & 1.8 & & & $\frac{5}{3}$ \\
\hline $14 \cdot 1-14$ & 14-Jun-94 & $\overline{3}$ & 24 & 5.6 & 0.12 & & & & & & & & & & & 13.5 & 7.16 & 3 \\
\hline $14-1-11$ & 02-Jun-95 & $\sqrt{3}$ & 24 & 4.7 & $<0.069$ & 0.06 & 80.9 & $\overline{0.3}$ & $<0 . \overline{05}$ & 38.8 & 8.89 & 3.52 & 0.1 & 0,57 & 187 & 14 & 6.99 & $\overline{3}$ \\
\hline $9-0.12$ & $14-J u n-94$ & $\overline{0}$ & 15.5 & 5.1 & 0.4 & & & & & & & & & & & 13.6 & & 3 \\
\hline $14-1-14$ & 02.Jun-95 & $\overline{3}$ & 24 & 5.6 & & 0.06 & & & & & & & & & & & & 3 \\
\hline
\end{tabular}

\section{Landfoll Leachale Plume}

\begin{tabular}{|c|c|c|c|c|c|c|c|c|c|c|c|c|c|}
\hline $\cos n$ & 0.31467 & 0.11917 & 46.229 & 5.9603 & 0.205 & 26.75 & 8.565 & 0.633 & 0.024 & 0.219 & 33.508 & 13.9625 & 7.208 \\
\hline count & 21 & 12 & 14 & 14 & 14 & 6 & 7 & 14 & 14 & 14 & 13 & 8 & 6 \\
\hline ed. & 0.20383 & 0.154 & 30.37 & 12.696 & 0.3069 & 10.961 & 0.498 & 1.043 & 0.039 & 0.222 & 49.899 & 0.82969 & 0.162 \\
\hline in & 0 & 0,04 & 18.3 & 0 & 0 & 11.2 & 7.94 & 0 & 0 & $\overline{0}$ & 1.8 & 12.7 & 6.99 \\
\hline $\max$ & $\overline{0.74}$ & 0.6 & 122 & 38,4 & 0.8 & 38.8 & 9.35 & 3,52 & 0,11 & 0.57 & 187 & 15.2 & 7.46 \\
\hline
\end{tabular}




\section{APPENDIX 12}

\section{PLFA AND PLATE COUNTS}

METHODS AND RESULTS

Note: references to "wells" in PLFA write-up should be "cores". 
Microbial Insights, Ine

201 Center Park Dr. Suite 1140

University of Waterloo

Knoxville, TN 37922

\section{University of Waterloo: PLFA and rRNA Analysis \\ September 25, 1995}

\section{Summary:}

Twenty-one sand samples were analyzed for phospholipid fatty acid and ribosomal RNA content. All samples contained relatively low biomass diverse microbial communities. It is apparent from the data that the observed trends in microbial community structure appear to be due more to a change in depth rather than the concentration of the available carbon source. However, differences in the level of biomass do appear to be dependant on carbon source availability, in that higher levels of biomass were detected in samples taken from within the creosote plume. The relative proportion of microeukaryotes such as fungi and protozoa, appears to dectease between a depth of 1.4 and 2.0 meters in all wells sampled. Between 2.0 and 2.6 meters a significant increase in the relative proportion of eukaryote PLFA is observed in wells 1 and 2, whereas it appears to remain constant in wells 3 and 4. In general, as the relative proportion of microeukaryotes changes, an opposing trend in the relative proportion of Gram positive and Gram negative bacteria is observed.

The relative proportions of PLFA found to be indicative for the presence of obligate anaerobes (such as sulfate reducing bacteria) and Actinomycete spp. appear to follow the same pattern and is highest at 1.4 meters in well 4 , peaks at 1.8 meters in wells 1 and 3 and at 2.0 meters in well 2.

The Gram negative community in well 1 (outside the plume) appear to have a slower tum over rate and are experiencing less environmental stress from either toxicity or starvation as compared to wells 2,3 and 4 (inside the plume).

Due to low biomass no rRNA was detected in any of the samples, therefore, no conclusions could be drawn regarding changes in cell activity.

\section{Biomass:}

The biomass measurement is the quantity of phospholipid fatty acids (PLFA) detected in the sample. Phospholipids are part of the intact cell membranes, thus the biomass is a measure of viable or potentially viable cells. As the cell nuptures, Phospholipids are attacked by enzymes resulting in a diglyceride fatty acid molecule representative of recently dead or lysed microorganisms.

The cell equivalent value is calculated from experiments with typical bacteria isolated from soil and water. This is based on $2.0 \times 10^{12}$ cells per gram dry weight of cells and $10^{8}$ pmols of phospholipid/gram dry weight of cells. This gives between $1.4 \times 10^{4}$ and $4.0 \times 10^{4}$ cells per pmol $\left(10^{-12}\right)$ of PLFA. The number of cells/gram of dry weight may vary and is dependant on the environmental conditions from which the microorganisms were recovered. 
Microbial Insights, Inc

201 Center Park Dr. Suite 1140

University of Waterloo

Knoxville, IN 37922

In general, the biomass for samples taken inside the plume is higher than that of samples taken outside the plume. This result is not surprising due to the availability of a carbon source (creosote and derivatives) within the plume. The biomass in wells 2 and 3 appear to follow a similar trend. The level of biomass in both wells increases between $1.4 \mathrm{~m}$ to $2.0 \mathrm{~m}$ prior to decreasing at $2.6 \mathrm{~m}$. Well 4 did not follow this observed tread in that the level of biomass in well 4 appears to decrease with increasing depth. Sample 4-1.4-M had the highest biomass of all the samples analyzed. Due to the low levels of biomass in well 1 no apparent trend could be detected.

\section{Community Strueture:}

The relative proportion of eukaryote PLFA indicative for the presence of microeukaryotes such as fungi and protozos, appears to decrease between a depth of 1.4 and 2.0 meters in all wells sampled. Between 2.0 and 2.6 meters a significant increase in the relative proportion of eukaryote PLFA is observed in wells 1 and 2, whereas it appears to remains constant in wells 3 and 4. In general, as the relative proportion of eukaryote PLFA changes an opposing trend in the relative proportion of terminally branched saturate PLFA and monoenoic PLFA is observed. Terminally branched saturate PLFA are representative of Gram positive bacteria but may also be found in the cell membranes of many sulfute reducing bacteria. Monoenoic PLFA are generally indicative for the presence of Gram negative bneteria

The relative proportions of branched monoenoic PLFA (indicative for the presence of obligate anaerobes auch as sulfite reducing bacteria) and mid chain branched saturate PLFA (found in Actinowycete spp., sulfinte reducing bacteria and certain Gram positive bacteria) appear to follow the same pattern. In wells 1 and 3 the relative proportion of both types of PLFA peak at 1.8 meters. In well 2 the relative proportion of both types of PLFA peak at 2.0 meters. On the other hand, the relative proportion of both types of PLFA is highest at 1.4 meters in well 4.

It is apparent from the above data that the observed trends in community structure appear to be due to a change in depth rather than the concentration of the available carbon source.

\section{Metabolic Status:}

In Gram negative bacteris, the monoenoics (16:1w7c \& 18:1w7c) are converted to cyclopropyl fatty acids (cy 17:0 \& cy19:0) as microbes move from a log to a stationary phase of growth (i.e. slowing of growth). This change is expressed in the two ratios cy 17:0/16:1 w7c)and cy19:0/18:1w7 $\bar{c}$. The ratios vary from organism to organism or environment to environment but usually will fall within the range of 0.05 (log phase) to 2.5 (tationary phase). When the ratios are summed the range is from 0 L to 5.0. An increase in cyclopropyl formation has also been associated with annerobic metaboliem.

The summation of the two ratios indieates that the Gram negative community within the samples from well 1 are in the stationary phase of growth. Furthermore, the Gram negative 
Microbial Insights, Inc

communities in well 1 have a slower tumover rate than those in wells 2,3 and 4 , i.e. wells within the plume. In wells 2, 3 and 4 the tumover rate of the Gram negative community appears to increase between a depth of 1.4 and 2.0 meters prior to decreasing at 2.6 meters.

Gram negative bacteria make trans futty acids to modify their cell membranes as protection toward changes in the environment such as toxicity or starvation. For example, the bacteria make w7t futty acids in the presence of toxic pollutants like phenol. Ratios (16:1w7t/16:1w7c and 18:1w7t/18:1w7c) greater than 0.1 have been shown to indicate the effects of starvation on bacterial isolates. The ringe is genernlly between 0.05 (healthy) to 0.3 (starved), or 0.1 (healthy) to 0.6 (starved) when the two are summed. Agnin, these calculations are for Gram negative bacteria but it is a fair ascumption that the reaults are applicable to the total community.

The summation of the two ratios indicates that the Gram negative communities within well 1 are experiencing little or no environmental stress from either toxicity or starvation. In contrast, the Gram negative communities from wells 2, 3 and 4 do appear to be experiencing environmental stress from either toxicity or starvation. Similar to the trend observed in stationary growth, the level of environmental stress in wells 2, 3 and 4 appears to decrease between a depth of 1.4 and 2.0 meters prior to increasing at 2.6 meters.

Ribosomal RNA (rRNA)

The levels of biomass for these samples typically fell in the region of $10^{5}$ cells/gram of dry sand. The detection limits for rRNA analysis lie at approximately $10^{5} \mathrm{cell} / \mathrm{gram}$ of $\mathrm{dry}$ sand. No rRNA was detected within these samples and no conchusions could be drawn regarding changes in cell activity. 
University of Waterloo

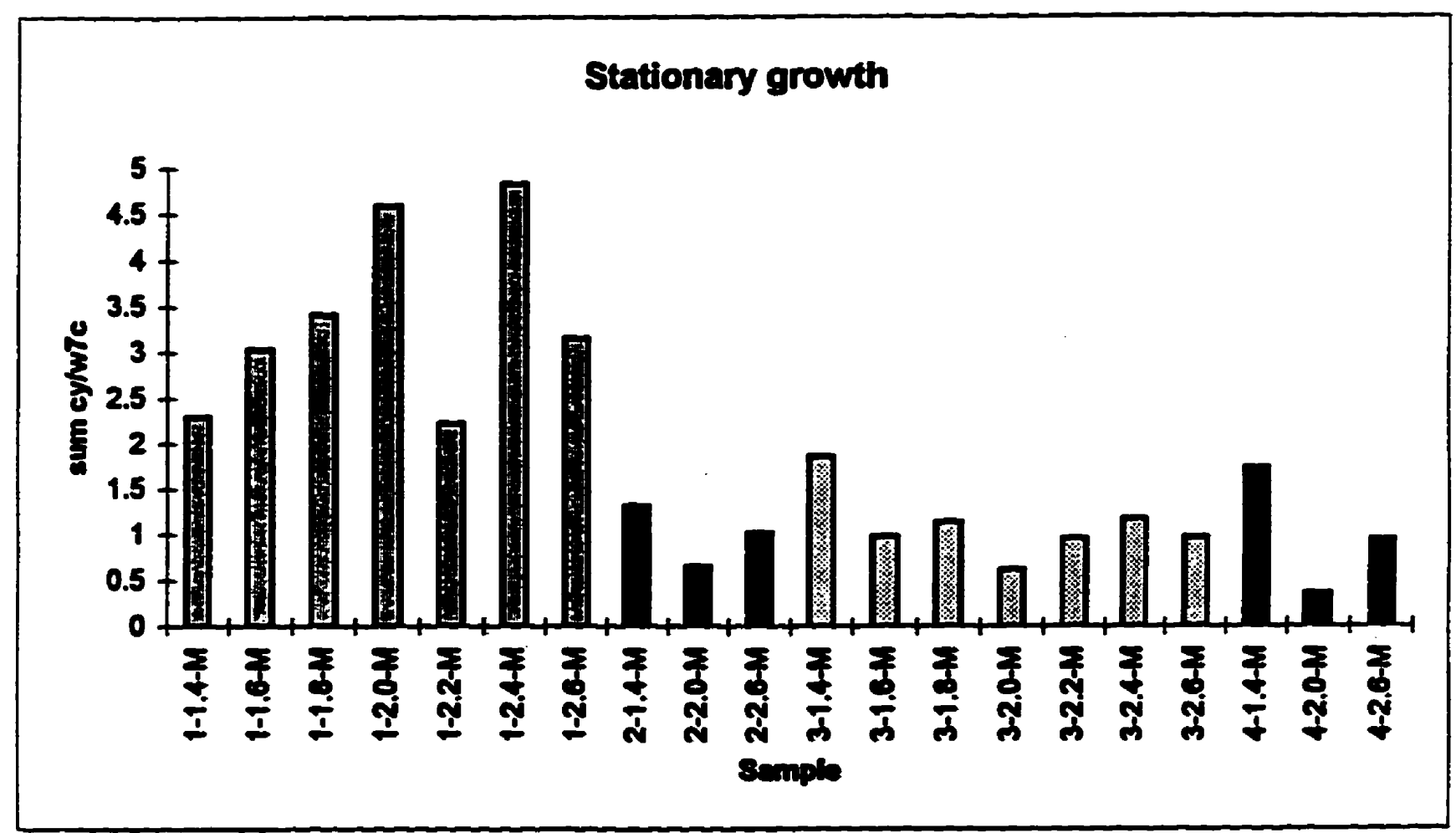

\section{Toxicity/Starvation}

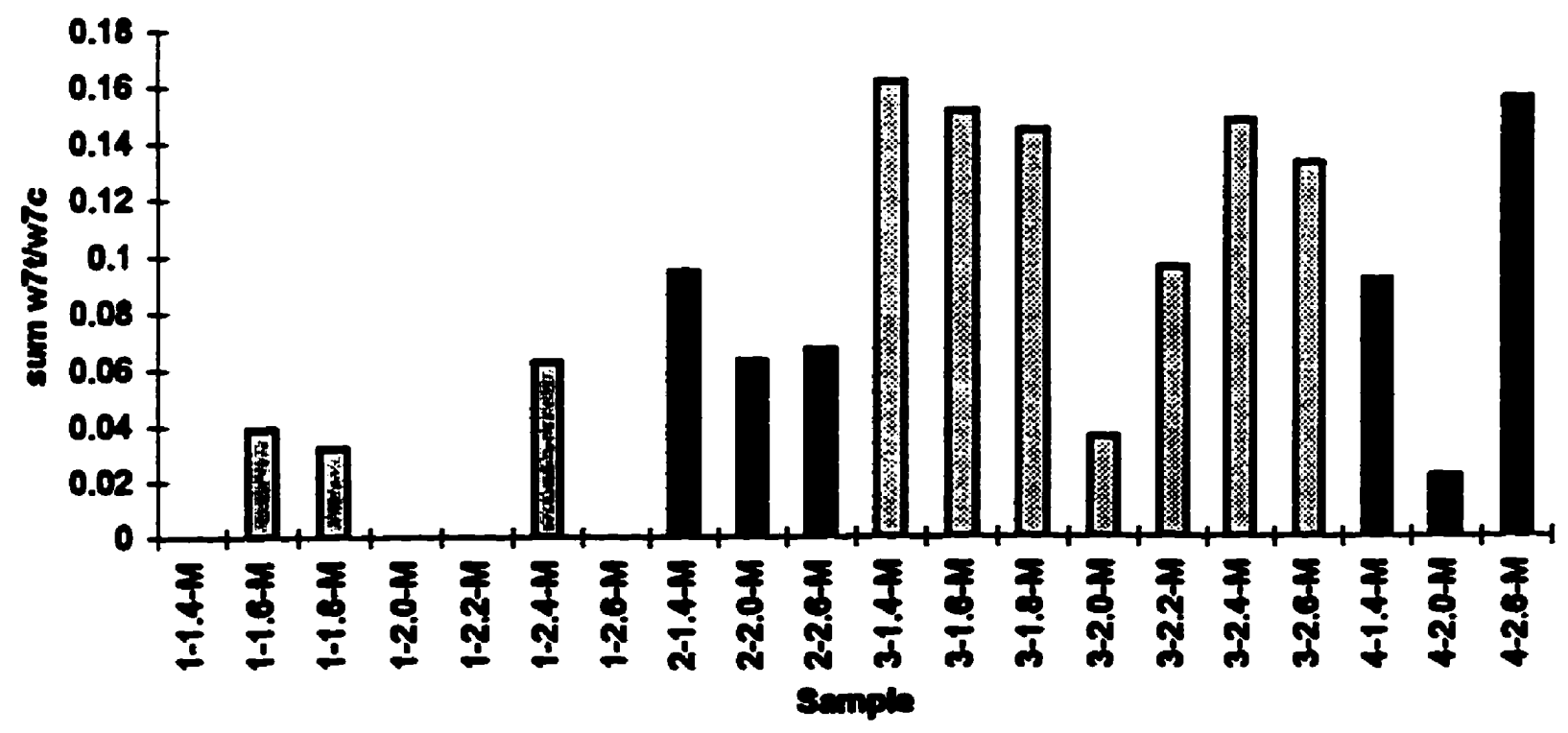


University of Waterloo
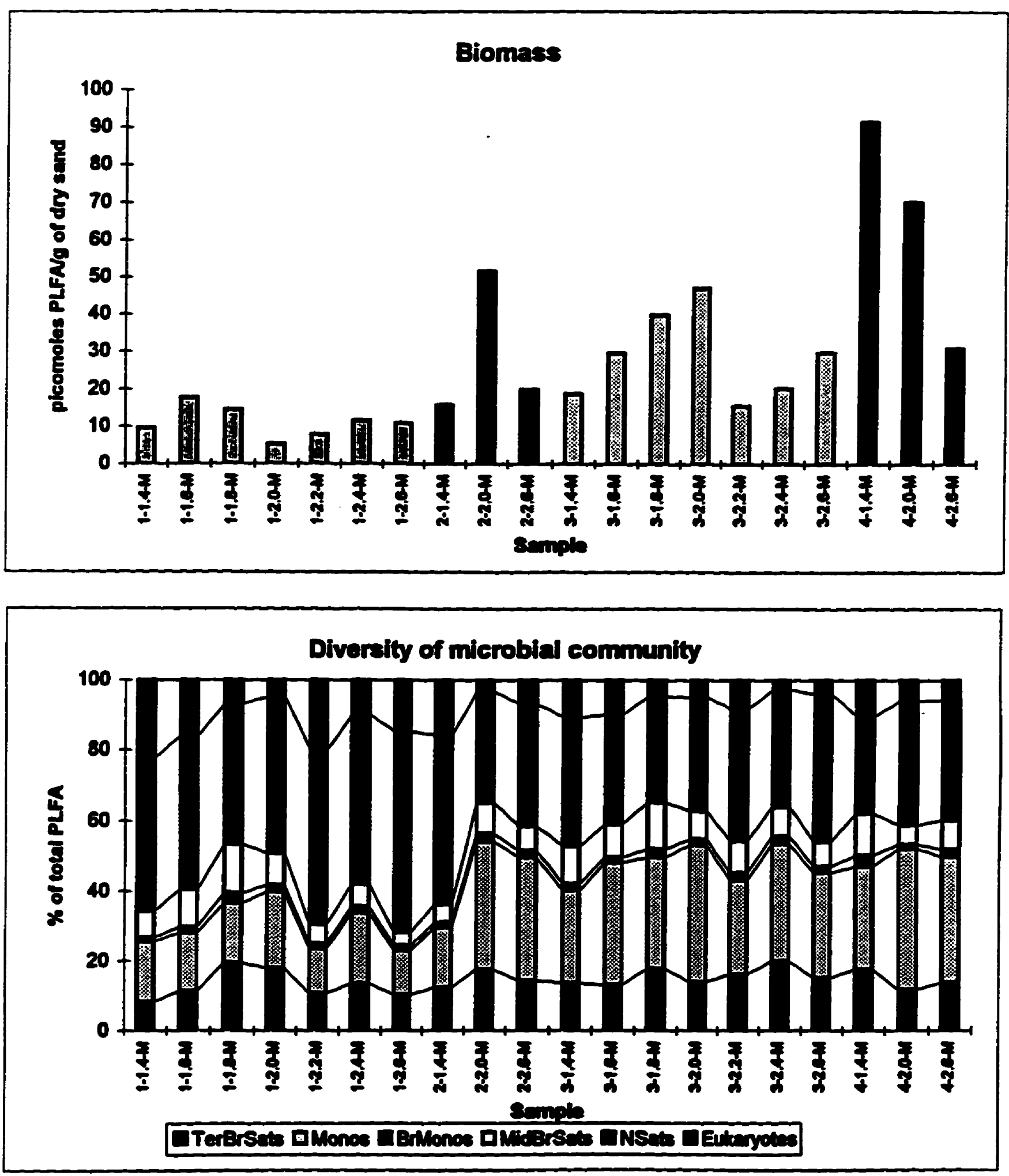
Microbial Insights. Inc.

Project Name

Mark King: University of Waterloo

Date Received 7/21/95

\begin{tabular}{lrrrrrr}
\hline \hline Sample Name & $1-1.4-M$ & $1-1.6-M$ & $1-1.8-M$ & $1-2.0-M$ & $1-2.2-M$ & $1-2.4-M$ \\
Amount of wet sand & 54.62 & 63.88 & 84.41 & 76.7 & 78.27 & 65.77 \\
Moisture content & $13.29 \%$ & $12.89 \%$ & $14.97 \%$ & $15.39 \%$ & $15.04 \%$ & $13.75 \%$ \\
Amount of dry sand & 47.36 & 55.65 & 71.78 & 64.90 & 66.50 & 56.73 \\
MI Identifier & $L 001.2$ & $L 001.3$ & $L 01.4$ & $L 001.5$ & $L 001.6$ & $L 001.7$ \\
Total Pico moles of PufA & 455 & 988 & 1.045 & 341 & 519 & 657 \\
\hline
\end{tabular}

Data Summary Sheet

\begin{tabular}{|c|c|c|c|c|c|c|}
\hline $\begin{array}{l}\text { picomoles PLFA/g of dry sand } \\
\text { Cells/o of dry sand } \\
\text { picomoles prokaryote PLFA } \\
\text { picomoles eukaryote PLFA } \\
\text { ratio prokaryote/oukaryote }\end{array}$ & $\begin{array}{r}9.6 \\
1.92 E+05 \\
7.5 \\
2.1 \\
4: 1\end{array}$ & $\begin{array}{r}17.8 \\
3.55 E+05 \\
14.9 \\
2.9 \\
5: 1\end{array}$ & $\begin{array}{r}14.6 \\
2.91 E+05 \\
13.6 \\
1.0 \\
13: 1\end{array}$ & $\begin{array}{r}5.3 \\
1.05 E+05 \\
5.0 \\
0.3 \\
19: 1\end{array}$ & $\begin{array}{r}7.8 \\
1.56 E+05 \\
6.2 \\
1.6 \\
4: 1\end{array}$ & $\begin{array}{r}11.6 \\
2.32 E+05 \\
10.4 \\
1.2 \\
9: 1\end{array}$ \\
\hline \multicolumn{7}{|l|}{ Methon } \\
\hline $\begin{array}{l}\text { cy17:0/16:1w7c } \\
\text { cy19:0/18:1w7c } \\
\text { Total }\end{array}$ & $\begin{array}{l}1.94 \\
0.35 \\
2.29\end{array}$ & $\begin{array}{l}2.49 \\
0.55 \\
3.04\end{array}$ & $\begin{array}{l}2.68 \\
0.72 \\
3.40\end{array}$ & $\begin{array}{l}3.74 \\
0.85 \\
4.59\end{array}$ & $\begin{array}{l}1.70 \\
0.52 \\
2.22\end{array}$ & $\begin{array}{l}4.05 \\
0.78 \\
4.83\end{array}$ \\
\hline $\begin{array}{l}16: 1 w 7 t / 16: 1 w 7 c \\
18: 1 w 7 t / 18: 1 w 7 c \\
\text { Total }\end{array}$ & $\begin{array}{l}0.00 \\
0.00 \\
0.00\end{array}$ & $\begin{array}{l}0.04 \\
0.00 \\
0.04\end{array}$ & $\begin{array}{l}0.03 \\
0.00 \\
0.03\end{array}$ & $\begin{array}{l}0.00 \\
0.00 \\
0.00\end{array}$ & $\begin{array}{l}0.00 \\
0.00 \\
0.00\end{array}$ & $\begin{array}{l}0.00 \\
0.06 \\
0.06\end{array}$ \\
\hline \multicolumn{7}{|c|}{ Com of Total PLFA) } \\
\hline TerBrSats & 8.3 & 11.3 & 19.4 & 18.0 & 10.6 & 13.4 \\
\hline Monos & 17.4 & 16.8 & 17.1 & 21.8 & 12.9 & 20.2 \\
\hline BrMonos & 1.0 & 1.7 & 2.8 & 2.0 & 1.3 & 1.8 \\
\hline MidBrSats & 7.6 & 10.7 & 14.0 & 9.1 & 5.6 & 6.5 \\
\hline NSats & 43.6 & 43.4 & 39.6 & 44.1 & 49.6 & 47.9 \\
\hline Eukaryotes & 22.2 & 16.1 & 6.9 & 5.0 & 20.0 & 10.0 \\
\hline
\end{tabular}

NA: Not Analyzed

NC: Nor Calculatod

ND: Not Detected 
Microbial Insights. Inc.

Project Name

Date Received

Mark King:

7/21/95

\begin{tabular}{lrrrrrr}
\hline \hline Sample Name & $1-2.6-M$ & $2-1.4-M$ & $2-2.0-M$ & $2-2.6-M$ & $3-1.4-M$ & $3-1.6-M$ \\
Amount of wet sand & 60.64 & 49.22 & 62.08 & 60.08 & 60.37 & 60.45 \\
Moisture content & $15.59 \%$ & $15.66 \%$ & $15.33 \%$ & $15.57 \%$ & $16.08 \%$ & $16.42 \%$ \\
Amount of dry sand & 51.18 & 41.51 & 52.56 & 50.73 & 50.66 & 50.52 \\
MI Identifier & $L 001.8$ & $L 001.9$ & $L 001.10$ & $L 001.11$ & $L 001.12$ & $L 001.13$ \\
Totel Pico moles of PLFA & 558 & 659 & 2.713 & 1.013 & 949 & 1.499 \\
\hline \hline
\end{tabular}

Data Summary Sheat

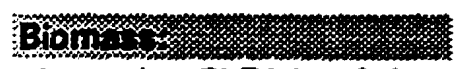

picomoles PLFA/g of dry and

Cells/g of dry sand

picomoles prokaryote PLFA

picomoles eukaryote PLFA

ratio prokaryote/eukaryote

$\begin{array}{lrrrrr}10.9 & 15.9 & 51.6 & 20.0 & 18.7 & 29.7\end{array}$

$2.18 E+053.18 E+05 \quad 1.03 E+063.99 E+053.75 E+05 \quad 5.93 E+05$

$\begin{array}{llllll}9.3 & 13.4 & 49.9 & 18.6 & 16.7 & 26.7\end{array}$

$\begin{array}{llllll}1.6 & 2.5 & 1.7 & 1.4 & 2.0 & 3.0\end{array}$

$\begin{array}{llllll}6: 1 & 5: 1 & 29: 1 & 20: 1 & 8: 1 & 9: 1\end{array}$

Hom

\section{cy17:0/16:1w7c \\ cy 19:0/18:1w7c \\ Total}

16:1w7t/16:1w7c

18:1w7t/18:1w7e

Total

\section{Cound}

TerBrSats

Monos

BrMonos

MidBrSats

NSats

Eukaryotes
2.65

0.51

3.16

0.00

0.00

0.00

\subsection{8}

0.64

1.02

0.04

0.03

0.07

\subsection{3}

0.29

1.31

0.33

0.32

0.65

0.05

0.05

0.10

0.05

0.01

0.08
1.42

0.44

1.86

0.10

0.06

0.16

14.4

35.3

1.9

7.0
17.4

36.8

2.3

8.7

31.5

3.3
34.5

6.8
13.7

26.5

2.0

10.7

36.3

10.8
0.62

0.36

0.98

0.13

0.03

0.15
NA: Not Analyzed

NC: Not Calculated

ND: Not Dotected
14.9
5.2

48.1

15.7

\begin{abstract}
15.7
\end{abstract}

$\begin{array}{rr}10.2 & 12.2 \\ 12.8 & 17.4 \\ 1.4 & 1.4 \\ 3.8 & 5.2 \\ 56.9 & 48.1 \\ 14.9 & 15.7\end{array}$

10.8 
Microbial Insights. Inc.

Project Name

Mark King:

Date Received

7/21/95

\begin{tabular}{lrrrrrr}
\hline \hline Sample Name & $3-1.8-M$ & $3-2.0-M$ & $3-2.2-M$ & $3-2.4-M$ & $3-2.6-M$ & $4-1.4-M$ \\
Amount of wet sand & 60.99 & 47.56 & 60.12 & 61.06 & 47.48 & 42.61 \\
Moisture content & $17.08 \%$ & $15.11 \%$ & $16.59 \%$ & $15.99 \%$ & $15.89 \%$ & $17.39 \%$ \\
Amount of dry sand & 50.57 & 40.38 & 50.14 & 51.29 & 39.93 & 35.20 \\
MI Identifier & $L 001.14$ & $L 001.15$ & $L 001.16$ & $L 001.17$ & $L 001.18$ & $L 001.19$ \\
Total Pico molse of PLFA & 2.018 & 1.902 & 779 & 1.040 & 1.188 & 3,216 \\
\hline \hline
\end{tabular}

Data Summary Shant

Find -

picomoles PLFA/g of dry sand

Cells/o of dry sand

$\begin{array}{llllll}39.9 & 47.1 & 15.5 & 20.3 & 29.8 & 91.4\end{array}$

picomoles prokaryote PLFA

picomoles oukaryote PLFA

ratio prokaryote/eukaryote

$7.98 E+05$ 9.42E+05 3.11E+05 4.05E+05 5.95E+05 1.83E+06

$\begin{array}{llllll}38.0 & 44.8 & 14.2 & 19.7 & 28.5 & 81.8\end{array}$

$\begin{array}{llllll}1.9 & 2.3 & 1.3 & 0.5 & 1.2 & 9.6\end{array}$

$\begin{array}{llllll}20: 1 & 19: 1 & 11: 1 & 36: 1 & 23: 1 & 9: 1\end{array}$

PIt:

cy 17:0/16:1w7c

cy19:0/18:1w7c

Total

$0.66 \quad 0.32$

0.40

0.23

0.45

0.99

0.48

0.29

0.54

0.93

0.51

0.74

1.14

0.61

0.95

1.16

0.96

1.73

16:1w7t/16:1 w7c

$\begin{array}{ll}0.09 & 0.03\end{array}$

0.10

0.08

0.07

0.04

18:1 w7t/18:1w7c

0.06

0.01

0.00

0.07

0.06

0.05

Total

$0.14 \quad 0.04$

0.10

0.15

0.13

0.09

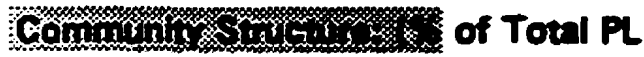

TerBrSats

Monos

BrMonos

MidBrSats

NSats

Eukaryotes

$\begin{array}{rrrrrr}17.8 & 13.9 & 16.1 & 20.0 & 15.2 & 17.6 \\ 32.0 & 39.4 & 27.2 & 33.6 & 30.1 & 29.5 \\ 2.3 & 1.7 & 2.0 & 2.2 & 1.9 & 3.1 \\ 13.4 & 8.0 & 9.3 & 8.3 & 7.1 & 12.0 \\ 29.9 & 32.2 & 37.1 & 33.1 & 41.6 & 27.2 \\ 4.7 & 4.9 & 8.3 & 2.7 & 4.2 & 10.5\end{array}$

NA: Not Analyzed

NC: Not Calculated

ND: Not Detected 
Microbial Insights. Inc.

Project Name

Mark King:

Date Received 7/21/95

\begin{tabular}{lrrr}
\hline \hline Sample Name & $4-2.0-M$ & $4-2.6-M$ & Filter blank \\
Amount of wet sand & 52.00 & 50.9 & - \\
Moisture content & $16.13 \%$ & $16.22 \%$ & - \\
Amount of dry sand & 43.61 & 42.64 & - \\
MI Identifier & $L 001.20$ & $L 001.21$ & Loo1.1 \\
Total Pico moles of PLFA & 3.054 & 1.319 & 178 \\
\hline \hline
\end{tabular}

Date Summery Sheet

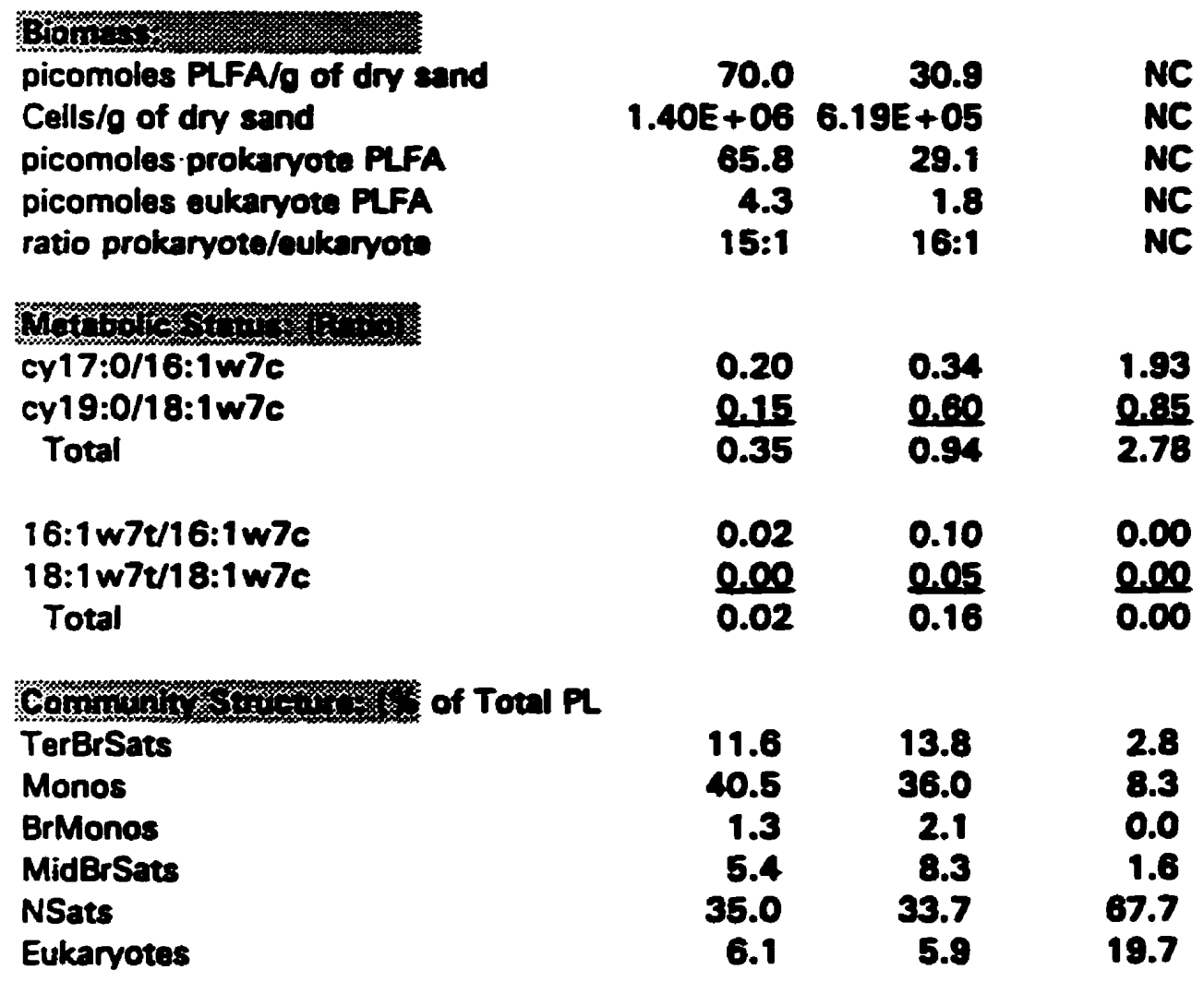

NA: Not Analyzed

NC: Not Calculated

ND: Not Detected 
Microbial Insights. Inc.

Project Name

Date Received

Mark King: University of Waterloo

7/21/95

\begin{tabular}{lrrrrrr}
\hline \hline Sample Name & $1-1.4-M$ & $1-1.6-M$ & $1-1.8-M$ & $1-2.0-M$ & $1-2.2-M$ & $1-2.4-M$ \\
Amount of wet sand & 54.62 & 63.88 & 84.41 & 76.7 & 78.27 & 65.77 \\
Moisture content & $13.29 \%$ & $12.89 \%$ & $14.97 \%$ & $15.39 \%$ & $15.04 \%$ & $13.75 \%$ \\
Amount of dry sand & 47.36 & 55.65 & 71.78 & 64.90 & 66.50 & 56.73 \\
MI Identifier & $L 001.2$ & $L 001.3$ & $L 001.4$ & $L 001.5$ & $L 001.6$ & $L 001.7$ \\
Total Pico moles of PLFA & 455 & 988 & 1.045 & 341 & 519 & 657 \\
\hline \hline
\end{tabular}

Analytical Report

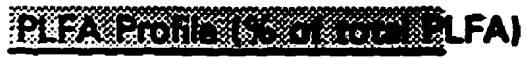

Terminally Branched Saturates (TorBrSats)

$\begin{array}{lrrrrrrr}i 14: 0 & 13.664 & 0.2 & 0.0 & 0.0 & 0.0 & 0.0 & 0.0 \\ i 15: 0 & 14.643 & 1.3 & 3.2 & 6.1 & 5.8 & 3.8 & 4.1 \\ \text { a15:0 } & 14.721 & 1.7 & 3.2 & 6.2 & 5.6 & 3.4 & 3.3 \\ i 16: 0 & 15.628 & 1.2 & 2.0 & 3.1 & 3.3 & 1.7 & 3.1 \\ i 17: 0 & 16.630 & 1.2 & 1.1 & 1.7 & 1.2 & 0.6 & 1.2 \\ \text { a17:0 } & 16.704 & 1.8 & 1.5 & 2.0 & 1.5 & 1.1 & 1.3 \\ i 18: 0 & 17.639 & \underline{0.8} & \underline{0.3} & \underline{0.5} & \underline{0.5} & \underline{0.0} & \frac{0.5}{13.4}\end{array}$

Monoenoics (Monos)

16:1w9c

$16: 1 w 7 c$

$16: 1 w 7 t$

$16: 1 w 5 c$

cy 17:0

$17: 1$

$18: 1 w 7 c$

$18: 1 w 7 t$

$18: 1 w 5 c$

cy19:0
15.702

15.739

15.776

15.851

16.816

17.114

17.752

17.808

17.864

18.818
0.6

2.9

0.0

0.7

5.6

0.6

5.0

0.0

0.2

17.8
1.0

2.7

0.1

0.7

6.8

0.6

3.1

0.0

0.1

1.7

16.8
1.5

2.5

0.1

0.9

6.8

0.9

2.5

0.0

0.1

1.8

17.1
1.4

2.6

0.0

0.8

8.9

0.8

3.2

0.0

0.4

2.7

21.8
0.0

3.0

0.0

0.5

5.1

0.5

2.4

0.0

0.2

1.2

12.9
1.1

2.4

0.0

0.6

9.7

0.5

3.2

0.2

0.0

2.5

20.2

Branched Monoenoics (BrMonos)

\section{i15:1}

a15:1

i17:1 w7e

br19:1
14.447

14.525

16.333

18.091

$\begin{array}{llll}0.0 & 0.4 & 1.0 & 0.5 \\ 0.0 & 0.4 & 0.5 & 0.3 \\ 0.7 & 0.7 & 1.1 & 1.0 \\ 0.4 & 0.2 & 0.3 & 0.2 \\ 1.0 & 1.7 & 2.8 & 2.0\end{array}$

0.6

0.0

0.7

0.0

1.3
0.6

0.3

0.8

0.0 
Microbial Insights. Inc.

Project Name

Date Received
Mark King: University of Waterloo 7/21/95

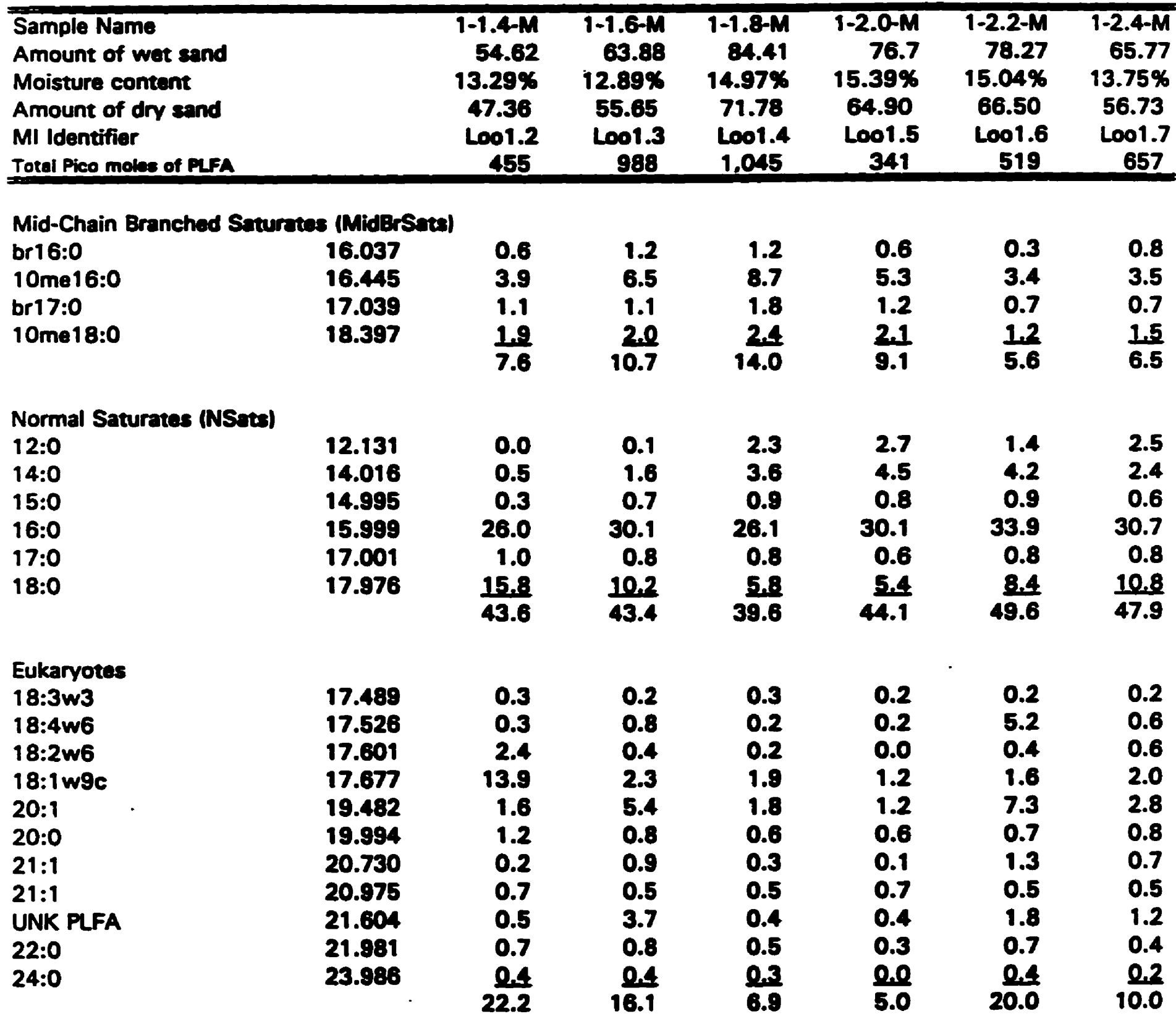


Microbial Insights. Inc.

Project Name

Mark King:

Date Received

7/21/95

\begin{tabular}{lrrrrrr}
\hline \hline Sample Name & $1-2.6-M$ & $2-1.4-M$ & $2-2.0-M$ & $2-2.6-M$ & $3-1.4-M$ & $3-1.6-M$ \\
Amount of wet sand & 60.64 & 49.22 & 62.08 & 60.08 & 60.37 & 60.45 \\
Moisture content & $15.59 \%$ & $15.66 \%$ & $15.33 \%$ & $15.57 \%$ & $16.08 \%$ & $16.42 \%$ \\
Amount of dry sand & 51.18 & 41.51 & 52.56 & 50.73 & 50.66 & 50.52 \\
MI Identifiet & $L 001.8$ & $L 001.9$ & $L 001.10$ & $L 001.11$ & $L 001.12$ & $L 001.13$ \\
Total Pico moles of PLFA & 558 & 659 & 2.713 & 1.013 & 949 & 1.499 \\
\hline
\end{tabular}

Analytical Report

DEris

Terminally Branched Saturates (TorBr

i1 4:0

i15:0 $\quad 14.643$

a15:0 $\quad 14.721$

i16:0

15.628

i17:0

16.630

a17:0

16.704

i18:0

17.639

$\begin{array}{rrrrrr}0.0 & 0.0 & 0.0 & 0.2 & 0.3 & 0.4 \\ 3.6 & 4.6 & 7.5 & 6.9 & 3.9 & 4.0 \\ 3.1 & 3.4 & 4.8 & 3.0 & 3.3 & 3.2 \\ 1.7 & 1.8 & 2.3 & 1.6 & 2.5 & 2.0 \\ 0.6 & 0.8 & 1.2 & 1.0 & 1.4 & 1.5 \\ 0.9 & 1.2 & 1.5 & 1.5 & 1.7 & 1.5 \\ 0.2 & 0.4 & 0.2 & 0.2 & 0.6 & \frac{0.3}{0.5} \\ 10.2 & 12.2 & 17.4 & 14.4 & 13.7 & 13.0\end{array}$

Monoenoics (Monos)

16:1 w9c

15.702

16:1w7c

15.739

16:1 1 7t

15.776

$16: 1$ w5c

15.851

cy17:0

16.816

17:1

17.114

18:1 w7c

17.752

18:1 w7t

17.808

18:1 w5c

17.864

cy19:0

18.818

$\begin{array}{rrr}0.7 & 1.0 & 1.4 \\ 2.1 & 4.7 & 18.6 \\ 0.0 & 0.2 & 0.9 \\ 0.4 & 1.0 & 2.1 \\ 5.5 & 4.9 & 6.1 \\ 0.4 & 0.4 & 0.5 \\ 2.3 & 3.8 & 5.1 \\ 0.0 & 0.2 & 0.1 \\ 0.2 & 0.0 & 0.2 \\ 1.2 & 1.1 & 1.7 \\ 12.8 & 17.4 & 36.8\end{array}$

1.4

1.2

1.4

1.3

0.8

17.8

$5.8 \quad 12.9$

2.1

0.7

0.6

1.6

2.8

1.4

1.6

6.1

6.7

8.3

8.0

0.5

0.3

0.6

0.5

3.5

5.5

6.4

0.1

0.1

0.3

0.2

0.1

0.3

0.3

$2.3 \quad 2.4 \quad 2.3$

$\frac{1.7}{36.8}$

35.3

26.5

35.1

Branched Monoenoics (BrMonos)

$\begin{array}{llllllll}\text { i15:1 } & 14.447 & 0.7 & 0.3 & 0.6 & 0.3 & 0.4 & 0.2 \\ \text { a15:1 } & 14.525 & 0.0 & 0.2 & 0.6 & 0.3 & 0.2 & 0.2 \\ \text { i17:1w7c } & 16.333 & 0.7 & 0.8 & 1.1 & 1.1 & 1.1 & 1.0 \\ \text { br19:1 } & 18.091 & \underline{0.0} & \underline{0.1} & \underline{0.1} & \underline{0.1} & \underline{0.3} & \frac{0.1}{1.5}\end{array}$


Microbial Insights. Inc.

Project Name

Date Received

Mark King:

7/21/95

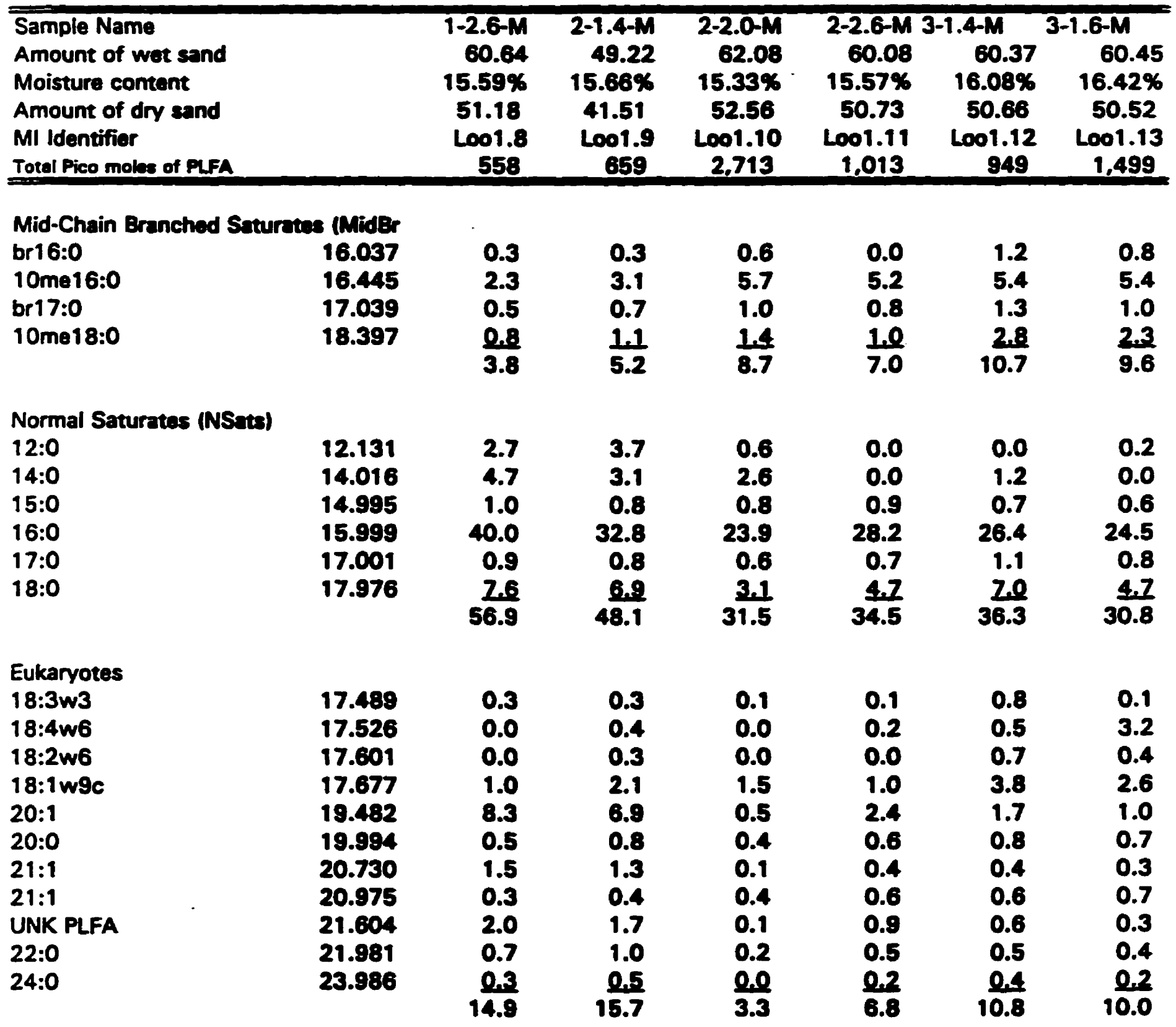


Microbial Insights. Inc.

Project Name

Date Received

Mark King:

7/21/95

\begin{tabular}{lrrrrrr}
\hline \hline Sample Name & $3-1.8-M$ & $3-2.0-M$ & $3-2.2-M$ & $3-2.4-M$ & $3-2.6-M$ & $4-1.4-M$ \\
Amount of wet sand & 60.99 & 47.56 & 60.12 & 61.06 & 47.48 & 42.61 \\
Moisture content & $17.08 \%$ & $15.11 \%$ & $16.59 \%$ & $15.99 \%$ & $15.89 \%$ & $17.39 \%$ \\
Amount of dry sand & 50.57 & 40.38 & 50.14 & 51.29 & 39.93 & 35.20 \\
MI Identifier & $L 001.14$ & $L 001.15$ & $L 001.16$ & $L 001.17$ & $L 001.18$ & $L 001.19$ \\
Total Pico moles of PLFA & 2.018 & 1,902 & 779 & 1.040 & 1.188 & 3,216 \\
\hline \hline
\end{tabular}

Analytical Report

PNA

Terminally Branched Saturates (TerBr

i14:0

i15:0

a15:0

i16:0

i17:0

a17:0

i18:0

Monoenoics (Monos)

16:1w9c

16:1w7c

$16: 1 w 7 t$

$16: 1 w 5 c$

cy17:0

$17: 1$

18:1w7c

18:1w7t

$18: 1 w 5 c$

cy 19:0
13.664

14.643

14.721

15.628

16.630

16.704

17.639

15.702
15.739
15.776
15.851
16.816
17.114
17.752
17.808
17.864
18.818
1.1

6.0

4.7

2.4

1.5

1.8

0.3

17.8

1.6

12.4

1.1

1.9

8.2

0.5

4.0

0.2

0.3

$\frac{1.9}{32.0}$
0.5

6.0

3.1

2.0

1.1

1.2

0.1

13.9
0.0

8.2

3.4

1.7

1.4

1.4

0.0

16.1
1.0

20.6

0.5

1.9

6.5

0.3

6.4

0.1

0.2

1.9

39.4
0.9

12.3

1.2

2.4

5.0

0.5

2.6

0.0

1.1

$\frac{1.4}{27.2}$
0.6

11.1

4.0

1.5

1.2

1.5

2.0

20.0
0.6

6.7

3.6

1.7

1.1

1.4

2.0

15.2
0.0

5.8

4.8

2.1

1.7

2.4

0.8

17.6

Branched Monoenoics (BrMonos)

i15:1

a15:1

i17:1 w7c

br19:1
14.447

14.525

16.333

18.091
0.5

0.5

1.2

0.2

2.3
0.4

0.3

0.8

0.1
1.3

18.8

1.4

3.4

4.3

0.4

2.0

0.1

0.0

1.8

33.6
1.4

14.0

1.0

2.6

6.3

0.3

2.9

0.2

0.0

1.5

30.1
1.8

5.8

0.3

2.1

5.7

0.7

7.0

0.3

0.7

5.2

29.5 
Microbial Insights. Inc.
Project Name
Mark King:
Date Received
7/21/95

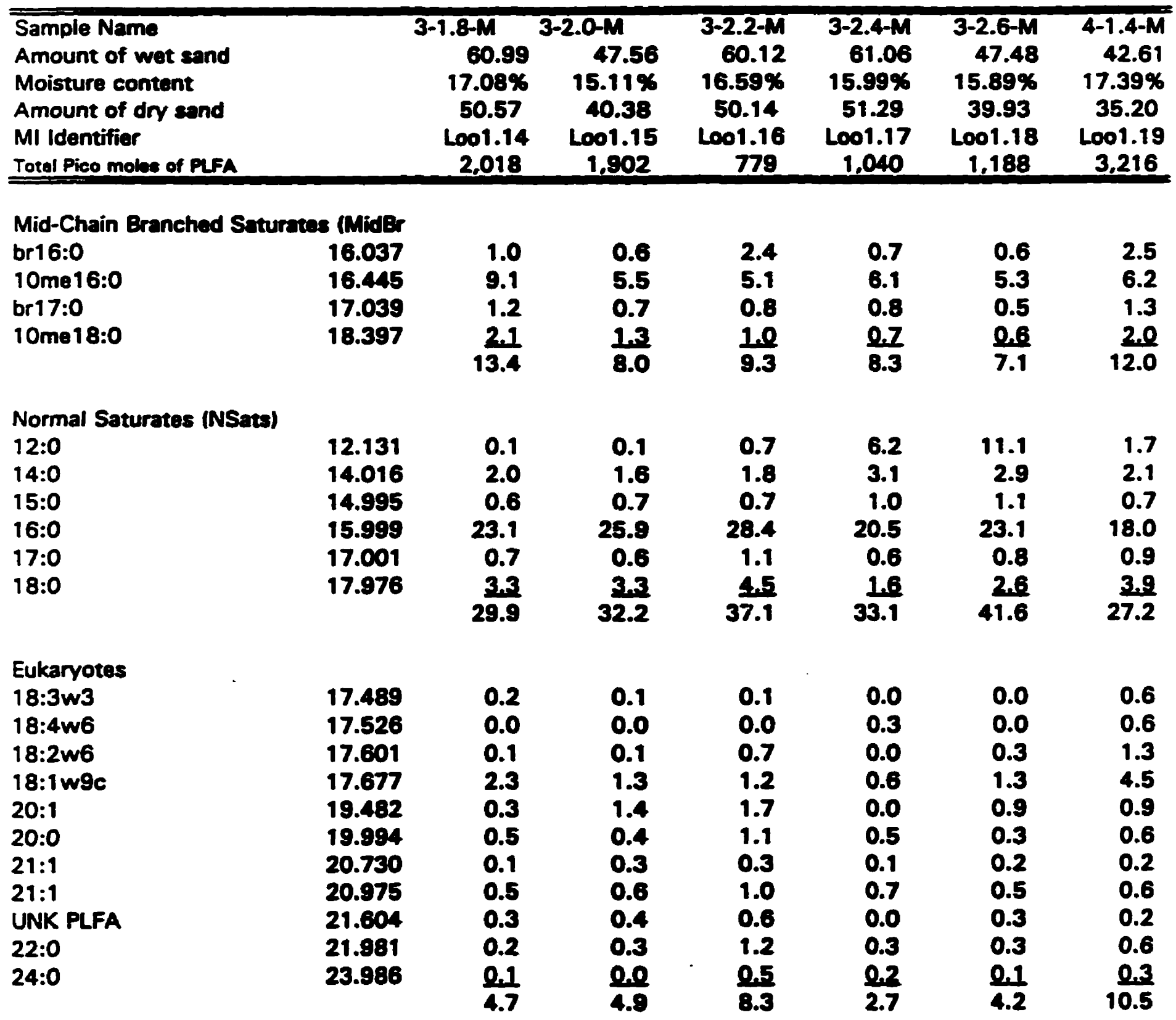


Microbial Insights. Inc.

Project Name

Mark King:

Date Received

7/21/95

\begin{tabular}{lrrr}
\hline \hline Sample Name & $4-2.0-M$ & $4-2.6-M$ & Filter blank \\
Amount of wet sand & 52.00 & 50.9 & $:$ \\
Moisture content & $16.13 \%$ & $16.22 \%$ & $:$ \\
Amount of dry sand & 43.61 & 42.64 & $:$ \\
MI ldentifier & $L 001.20$ & Lool.21 & Loo1.1 \\
Total Pico moles of PLFA & 3.054 & 1.319 & 178 \\
\hline \hline
\end{tabular}

Analytical Report

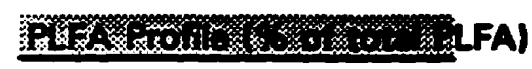

Terminally Branched Saturntes (TerBr

i14:0

i15:0

13.664

a15:0

14.643

i16:0

14.721

15.628

i17:0

16.630

a17:0

16.704

i18:0

17.639

$\begin{array}{rrr}0.0 & 0.0 & 0.7 \\ 5.2 & 7.1 & 0.7 \\ 3.2 & 3.0 & 0.0 \\ 1.3 & 1.4 & 0.9 \\ 0.7 & 1.1 & 0.6 \\ 1.0 & 1.4 & 0.0 \\ 0.1 & \underline{0.0} & \underline{0.0} \\ 11.6 & 13.8 & 2.8\end{array}$

Monoenoics (Monos)

$\begin{array}{lrrrr}16: 1 w 9 c & 15.702 & 0.8 & 1.3 & 0.0 \\ 16: 1 w 7 c & 15.739 & 23.7 & 18.0 & 1.6 \\ 16: 1 w 7 t & 15.776 & 0.5 & 1.8 & 0.0 \\ 16: 1 w 5 c & 15.851 & 1.6 & 3.5 & 0.0 \\ c y 17: 0 & 16.816 & 4.9 & 6.1 & 3.0 \\ 17: 1 & 17.114 & 0.3 & 0.3 & 0.5 \\ 18: 1 w 7 c & 17.752 & 7.6 & 2.9 & 1.3 \\ 18: 1 w 7 t & 17.808 & 0.0 & 0.2 & 0.0 \\ 18: 1 w 5 c & 17.864 & 0.1 & 0.1 & 0.7 \\ c y 19: 0 & 18.818 & 1.1 & 1.8 & 1.1 \\ & & 40.5 & 36.0 & 8.3\end{array}$

Branched Monoenoics (BrMonos)

$\begin{array}{lllll}\text { i15:1 } & 14.447 & 0.3 & 0.4 & 0.0 \\ \text { a15:1 } & 14.525 & 0.3 & 0.5 & 0.0 \\ \text { i17:1w7c } & 16.333 & 0.7 & 1.1 & 0.0 \\ \text { br19:1 } & 18.091 & 0.1 & 0.1 & 0.0 \\ & & 1.3 & 2.1 & 0.0\end{array}$


Microbial Insights. Inc.

Project Name

Mark King:

Date Received

7/21/95

\begin{tabular}{lrrr}
\hline \hline Sample Name & $4-2.0-M$ & $4-2.6-M$ & Fiter blank \\
Amount of wet sand & 52.00 & 50.9 & - \\
Moisture content & $16.13 \%$ & $16.22 \%$ & - \\
Amount of dry sand & 43.61 & 42.64 & - \\
MI Identifier & L001.20 & L001.21 & Loo1.1 \\
Total Pico molec of PLFA & 3.054 & 1.319 & 178 \\
\hline \hline
\end{tabular}

$\begin{array}{lllll}\text { Mid-Chain Branched Saturates (MidBr } & & & \\ \text { br16:0 } & 16.037 & 0.4 & 0.5 & 1.6 \\ \text { 10me16:0 } & 16.445 & 3.6 & 6.3 & 0.0 \\ \text { br17:0 } & 17.039 & 0.6 & 0.6 & 0.0 \\ \text { 10me18:0 } & 18.397 & 0.8 & 0.9 & 0.0 \\ & & 5.4 & 8.3 & 1.6\end{array}$

Normal Saturates (NSats)

12:0

$14: 0$

15:0

16:0

$17: 0$

18:0

\section{Eukaryotes}

18:3w3

18:4w6

18:2w6

$18: 1 w 9 c$

20:1

20:0

21:1

21:1

UNK PLFA

22:0

24:0
12.131

14.016

14.995

15.999

17.001

17.976

17.489
17.526
17.601
17.677
19.482
19.994
20.730
20.875
21.604
21.881
23.986

$\begin{array}{rrr}2.7 & 2.9 & 0.7 \\ 1.5 & 2.4 & 2.8 \\ 0.7 & 1.0 & 0.8 \\ 26.7 & 24.0 & 42.9 \\ 0.5 & 0.7 & 1.9 \\ 3.0 & 2.7 & 18.5 \\ 35.0 & 33.7 & 67.7\end{array}$

0.1

0.1

0.9

0.1

0.3

0.2

1.6

1.3

0.2

1.5

2.5

2.2

0.4

0.4

0.3

0.6

0.3

0.1

6.1
1.3

7.0

$0.6 \quad 0.9$

$0.5 \quad 1.2$

$\begin{array}{ll}0.7 & 0.0\end{array}$

$0.5 \quad 2.8$

$0.5 \quad 1.3$

$0.0 \quad 0.0$

$5.9 \quad 19.7$ 
Microbial Insights, Inc.

Quality Assurance Report

Company: Mark King, University of Waterloo

Twenty-one sand samples were received on 7/21/95. These sumples were analyzed for PLFA and TRNA content.

These samples were analyzed under the U.S. EPA Good Laboratory Practice Standards; Toxic Substances Control Act (40 CFR part 790).

All instruments used in the analysis were calibrated and operated within acceptable ranges. The instruments were calibrated according to Microbial Insights, Inc. Standard Operating Procedures (SOP) EQ1.

Inspection Date of Pffe I of the analysis Instrument calibration occurred 5/25/95

by:

Phase/II consisted of the processing of samples sccording to sop's B\&D

HPGC1. Inspection of Phase II occurred on 8/17/95 by:

The following solvents were used during the analysis and wote free of contamination.

Chloroform Lot \#s BK402

Methanol Lot \#'s BJ946

Acetone Lot \#'s BJ534

$\mathrm{H}_{2} \mathrm{O}$ Buffer Lot \# PB0049

All data generated through this analysis was acceptable

All required analysis holding times were met.

Samples arrival conditions were acceptable.

No QC or analytical problems were encountered. 


\section{PLATE COUNT METHODS}

(analysis and write-up by Dr. Barb Butler)

Aliquots of sand samples that were analyzed by Microbial Insights for PLFA were also analyzed for total plate counts. As part of this analysis moisture content was also determined. Triplicate samples of wet sand were dispensed into pre-dried aluminum pans, weighed, then dried overnight at $100^{\circ} \mathrm{C}$ and reweighed. Mean values were as follows.

\begin{tabular}{ccc}
\hline Sample & Moisture content (\%) & standard deviation \\
\hline $2-1.4$ & 15.99 & 0.08 \\
$1-2.0$ & 16.05 & 0.32 \\
$2-2.6$ & 15.22 & 0.08 \\
$3-1.4$ & 17.07 & 0.20 \\
$3-2.0$ & 15.18 & 0.33 \\
$3-2.6$ & 15.98 & 0.06 \\
$4-1.4$ & 17.08 & 0.58 \\
$4-2.0$ & 16.16 & 0.28 \\
$4-2.6$ & 16.13 & 0.11 \\
\hline
\end{tabular}

To determine plate counts, a suspension of $10 \mathrm{~g}$ (wet wt) of sand in $90 \mathrm{~mL}$ of $0.1 \%$ $\mathrm{Na}_{4} \mathrm{P}_{2} \mathrm{O}_{7} \cdot 1 \mathrm{H}_{2} \mathrm{O}$ (pH 7.0) was shaken for $10 \mathrm{~min}$ on an orbital shaker operating at $400 \mathrm{rpm}$, then diluted in phosphate-buffered saline ( $1.18 \mathrm{~g} \mathrm{Na}_{2} \mathrm{HPO}_{3}, 0.22 \mathrm{~g} \mathrm{NaH}_{2} \mathrm{PO}_{4} \cdot \mathrm{H}_{2} \mathrm{O}, 8.5 \mathrm{~g} \mathrm{NaCl}$ per $\mathrm{L}, \mathrm{pH}$ 7.0) and spread on plates of R2A medium. Triplicate plates were prepared for each dilution. Plates were incubated aerobically at $10^{\circ} \mathrm{C}$ and counts were made at 40 days and are reported as colony forming units (CFUs)/g dwt in the following:

\begin{tabular}{ccc}
\hline Sample & CFU/g & s.d. \\
\hline $2-1.4$ & $1.1 \times 10^{4}$ & $2.1 \times 10^{3}$ \\
$2-2.0$ & $4.1 \times 10^{3 *}$ & $1.3 \times 10^{3}$ \\
$2-2.6$ & $3.9 \times 10^{2 *}$ & $2.0 \times 10^{2}$ \\
$3-1.4$ & $1.3 \times 10^{4}$ & $1.0 \times 10^{3}$ \\
$3-2.0$ & $1.2 \times 10^{4}$ & - \\
$3-2.6$ & $2.4 \times 10^{2 *}$ & $1.9 \times 10^{2}$ \\
$4-1.4$ & $6.9 \times 10^{3}$ & $3.5 \times 10^{2}$ \\
$4-2.0$ & $2.2 \times 10^{3 *}$ & $3.9 \times 10^{2}$ \\
$4-2.6$ & $1.2 \times 10^{3 *}$ & 0 \\
\hline
\end{tabular}

* estimate; some/all plates had fewer than 30 colonies

results are mean of triplicate plates, except 3-2.0: only two plates available due to lab accident 


\section{APPENDIX 13}

\section{AROMATIC ACIDS RESULTS}

\section{BACKGROUND}

A set of groundwater samples was collected on September 22, 1995, to evaluate for the presence of organic metabolites due to plume biodegradation. Aromatic acids were analyzed at the National Center for Bioremediation Research and Development at the University of Michigan under the supervision of $\mathrm{Dr}$. Jiasong Fang, and using methodology described by Barcelona et al. (1995). Aromatic acid results are provided in Table 1 and associated dissolved creosote compound results are provided in Table 2. 
Table 1. Aromatic acid results for groundwater samples collected September 22, 1995; concentrations in ug/L.

\begin{tabular}{|c|c|c|c|c|c|c|c|c|c|c|}
\hline $\begin{array}{l}\text { Location: } \\
\text { Depth (in below grede): }\end{array}$ & $\begin{array}{l}\sqrt{3}-0 \\
1.80 \\
\end{array}$ & $\begin{array}{l}\mathbf{N 3}-0 \\
3.00 \\
\end{array}$ & $\begin{array}{l}5 S 12 \\
2.93\end{array}$ & $\begin{array}{l}5527 \\
2.40\end{array}$ & $\begin{array}{l}5 S 27 \\
330\end{array}$ & $\begin{array}{l}9-1 \\
1.80\end{array}$ & $\begin{array}{c}9-1 \\
2.40\end{array}$ & $\begin{array}{c}9.1 \\
3.60\end{array}$ & $\begin{array}{l}18-1 \\
3.20 \\
\end{array}$ & $\begin{array}{c}\text { Procedure } \\
\text { blank }\end{array}$ \\
\hline Berzoic acid & 6 & 49.97 & 36.58 & 5294 & 9528.83 & 5324 & 18.14 & 25.86 & 19.85 & 0 \\
\hline 2-Methylbenzoic ecid & (0) & $\overline{7.48}$ & 0 & 292 & 0 & 3.42 & 0 & 0 & 4.58 & 0 \\
\hline Methyl salicutate & 27.82 & 93.36 & 0 & 0 & 2160.56 & 3.37 & 2494,39 & 232.52 & 1299.73 & 0 \\
\hline 3-Methylbenoic ecid & 0 & 12.77 & 16.86 & 297.56 & 5477.46 & 0 & 11.04 & 59.18 & 28.13 & 0 \\
\hline 4-Methvlbernoic ecid & 2.63 & 143 & 16.16 & 333.16 & 0 & 0 & 12.36 & 15.67 & 7.83 & $\overline{0}$ \\
\hline 2.6-Dimethylbernoicecid & 0 & 20.54 & 9.04 & 0 & 0 & 0 & 66.98 & 2.75 & 27.24 & $\overline{0}$ \\
\hline 2.5-Dimethylbempoic ecid & 3.39 & 25.59 & 10.57 & $\mathbf{0}$ & $\mathbf{0}$ & 5.78 & 7.85 & 2.01 & 11.79 & $\mathbf{0}$ \\
\hline 23/+3.5-Dimethylbereoic ecid & 0 & 32.95 & 2493 & 0 & 0 & 0 & 45.58 & 8.02 & 57.13 & 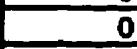 \\
\hline 2.4.6-Trimedhyloemoic ecid & 0 & 0 & 0 & 0 & 0 & 0 & 0 & 0 & 0 & 5 \\
\hline 3.4-Dimethylberzoic acid & 2.63 & 21.42 & 34.43 & 6636 & 1145.15 & 62.13 & 3723 & 9.9 & 52.52 & - \\
\hline
\end{tabular}

Table 2. Dissolved creosote results for groundwater samples collected September 22, 1995; concentrations in ug/L.

\begin{tabular}{|c|c|c|c|c|c|c|c|c|c|}
\hline $\begin{array}{l}\text { Loction: } \\
\text { Depth (m below erede): }\end{array}$ & $\begin{array}{l}\sqrt{3-0} \\
1.80\end{array}$ & $\begin{array}{l}\mathbf{N 3 - 0} \\
3.00\end{array}$ & $\begin{array}{l}\text { SS12 } \\
2.93\end{array}$ & $\begin{array}{l}\text { SS27 } \\
2.40\end{array}$ & $\begin{array}{l}5527 \\
3.30\end{array}$ & $\begin{array}{l}9-1 \\
1.80\end{array}$ & $\begin{array}{c}9-1 \\
2.40\end{array}$ & $\begin{array}{c}91 \\
3.60\end{array}$ & $\begin{array}{l}18-1 \\
320 \\
\end{array}$ \\
\hline m-xylene & 0 & & 0 & 18 & 0 & 0 & 0 & 1176 & 0 \\
\hline phenol & 0 & 0 & $\mathbf{0}$ & 0 & 0 & 0 & 0 & 0] & 0 \\
\hline o-cresol & 0 & 0 & 0 & 0 & 0 & 0 & 0 & 0 & 0 \\
\hline$p+m-c r e s o l$ & 0 & 0 & 0 & 0 & 0 & 0 & 0) & 0 & 0 \\
\hline 2.6dmp & 0 & 0 & 0 & 0 & 0 & 0 & 0 & 0 & 0 \\
\hline $2.4+2.5-\mathrm{dmp}$ & 0 & 0 & 0 & 0 & 0 & 0 & 0) & 0 & 0 \\
\hline 23-dmp & 0 & of & 0 & 0 & 0 & 0 & 0 & 0) & 0 \\
\hline 3.5-dmp & 0 & 0 & 0 & 0 & $\mathbf{0}$ & 0 & (0) & 0 & 0 \\
\hline naphthalere & 0 & 0 & 6682 & 3293 & 679 & 0 & 3037 & 8760 & 6050 \\
\hline indole $+2-\mathrm{mmph}$ & 0 & 0 & $13 \times 0$ & 461 & 211 & 0 & 20 & 849 & 0 \\
\hline l-m.naph & 0 & 0 & 562 & 256 & 80 & 0 & 386 & 376 & 0 \\
\hline biphenvl & 0 & 01 & 255 & 122 & 37 & 0 & 29 & 41 & 0 \\
\hline scengohthv'=1s & 0 & of & 0 & 9 & 0 & (0) & 0 & 0 & 0 \\
\hline acenaphliens & 0 & 0 & 1201 & 580 & 279 & 0 & 252 & 266 & $\mathbf{0}$ \\
\hline dibenzofurs & 0 & 0 & 650 & 355 & 169 & (0) & 0 & 0 & $\overline{0}$ \\
\hline fluorene & 0 & of & 347 & 159 & 73 & (0) & 0 & 0 & 0 \\
\hline pheonthrous & 0 & of & 258 & 0 & 0 & 0 & 0. & 0 & 0 \\
\hline enthrecene & 0 & of & 80 & 0 & 0 & 0 & 0 & 0. & 0 \\
\hline catbenzole & 0 & of & 375 & 118 & 0 & 0 & 0] & (0) & 0 \\
\hline fluorenthene & 0 & 0 & 24 & 0 & 0 & 0 & 01 & 0 & 0 \\
\hline pyrede & 0 & (0) & 20 & 0] & 0 & 0 & 01 & 0 & 0 \\
\hline
\end{tabular}

Note: see Appesdix 4 for Desection Limits 


\section{APPENDIX 14 \\ BIO3D INPUT FILES}

\section{BACKGROUND}

The program BIO3D was used to model a plume resembling that of dibenzofuran at the field site under two different conditions:1) solute does not degrade and 2) solute degradation is essentially instaneous in the presence of oxygen. The input file for these analyses is provided as Tables 1 , and results are shown graphically in Figure 5.2, in the main body of this document. The BIO3D source code is available from one of the developers: John Molson, University of Waterloo, Earth Science Department. 
Table 1. BIO3D data input file to simulate aerobic biodegradation of dibenzofuran at the field site, assuming that dibenzofuran is the only compound present. For simulation of a nonbiodegrading solute, the oxygen concentration below which biodegradation no longer occurs (THRESHOLD - 0) was set above background dissolved oxygen (>2.5 mg/L).

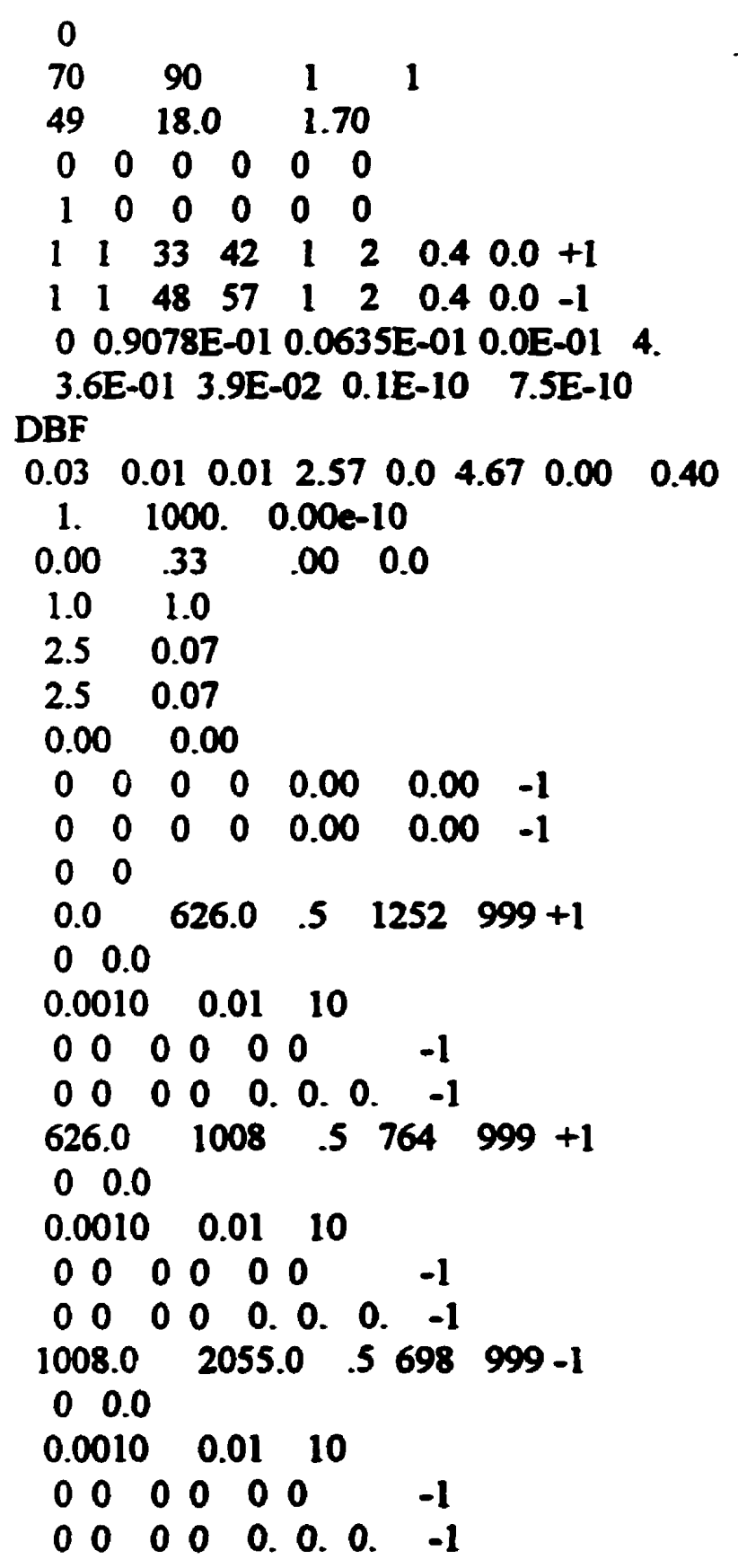

\author{
;KPRT \\ ;NEX,NEY,NEZ,\# of substrates \\ $; \mathbf{X}, \mathbf{Y L}, \mathbf{Z L}$ \\ ;source node ranges \\ ;BOUNDARY TYPE CODES \\ ;boundary patch 1 \\ ;boundary patch 2 \\ ;IVOPT, VX, VY, VZ,ROTA (m/d) \\ ;AL,ATH,ATV,DD \\ ;utils, uhs, uho,ros,ym, res, uibs, uins \\ ;substratel terms \\ ;RETO,retm,bm \\ ;B,PORM,PORS,DPW(DUAL-P) \\ ;UNDER-RELAXATION:ORG,OXY \\ ;BACKGROUND CONC. oxy-M \\ ;INIT.SOUR.CONC. oxy-M \\ ; threshold S-O \\ ;VARIABLE SUB BACKG \\ ;VARIABLE OXY BACKG \\ ;RESTART/FLOAT DT? 0=NO \\ ;TO,T1,DT,KTIME,KMOM (days) \\ ;KVREAD,ROTA \\ ;TOLS, TOLO, iter \\ ;source: il,i2,j1,j2,k1,122 \\ ;sink: i,j,kl,k2,PQ,cs,co \\ ;TO,T1,DT,KTMME,KMOM (days) \\ ;KVREAD,ROTA \\ ;TOLS,TOLO, iter \\ ;source: il,i2,j1,j2,k1,k2 \\ ;sink: $i, j, k 1, k 2, P Q, c s, c o$ \\ ;TO,T1,DT,KTIME,KMOM (days) \\ ;KVREAD,ROTA \\ ;TOLS, TOLO, iter \\ ;source: il,i2,jl, j2,k1,k2 \\ ;sink: i,j,kl,k2,PQ,cs,co
}

